\title{
Computational Study of Enantioselective Carboligation Catalyzed by Benzoylformate Decarboxylase
}

\author{
Ferran Planas $^{a}$, Michael J McLeish ${ }^{b}$ and Fahmi Himo ${ }^{a *}$
}

${ }^{a}$ Department of Organic Chemistry, Arrhenius Laboratory, Stockholm University, SE-10691, Stockholm (Sweden)

${ }^{b}$ Department of Chemistry and Chemical Biology, Indiana University-Purdue University Indianapolis, IN 46202, Indianapolis (USA)

\section{Table of contents}

1. Superposition of structures of different benzaldehyde binding modes

2. Superposition of structures of different acetaldehyde binding modes

3. Tables with calculated energies and energy corrections

4. Cartesian coordinates of optimized structures 
1. Superposition of structures of different benzaldehyde binding modes

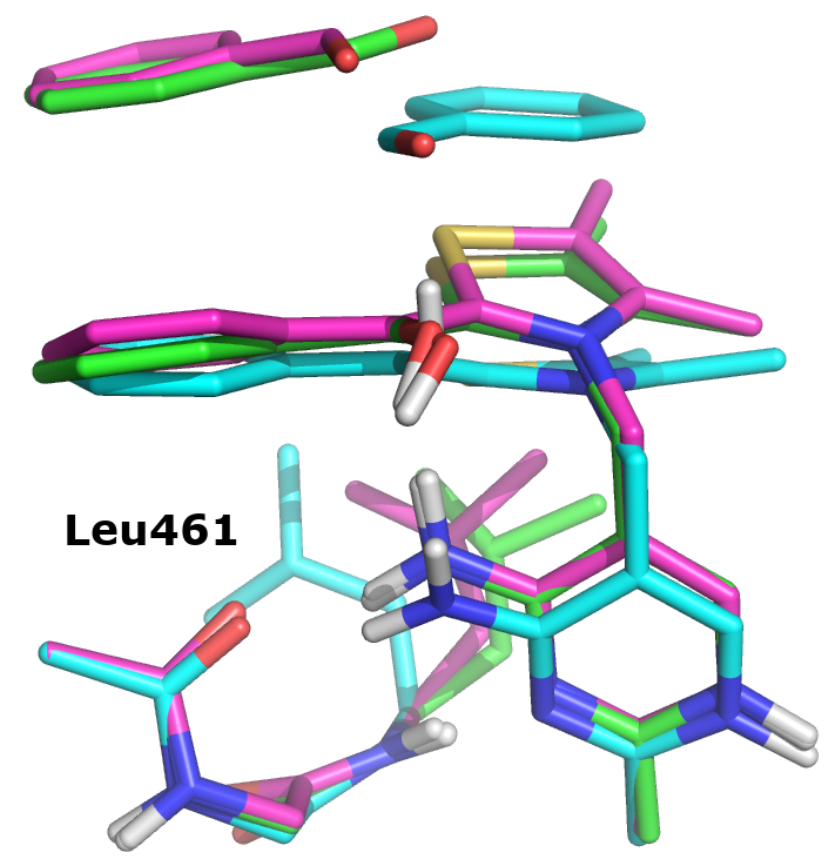

Figure S1. Superposition of the $\boldsymbol{E n}(\boldsymbol{B A})-\boldsymbol{R} 1$ (pink), $\boldsymbol{E n}(\boldsymbol{B} \boldsymbol{A})-\boldsymbol{S} 1$ (green) and $\boldsymbol{E n}(\boldsymbol{B A})-\boldsymbol{S} 2$ (blue) binding modes.

2. Superposition of structures of different acetaldehyde binding modes

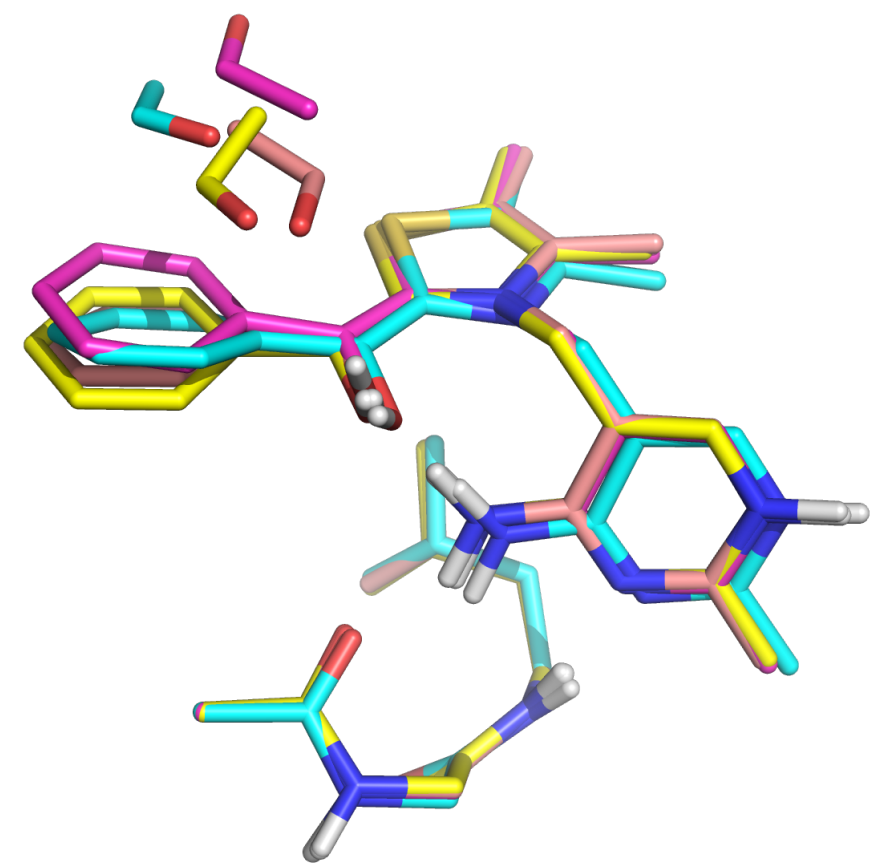

Figure S2. Superposition of the $\operatorname{En}(\boldsymbol{A A})-\boldsymbol{S} 1$ (blue), $\boldsymbol{E n}(\boldsymbol{A A})-\boldsymbol{S} 2$ (yellow), $\boldsymbol{E n}(\boldsymbol{A A})-\boldsymbol{R} 1$ (brown) and $\boldsymbol{E n}(\boldsymbol{A} \boldsymbol{A})-\boldsymbol{R} 2$ (pink) binding modes. 


\section{Tables with calculated energies and energy corrections}

Table S1. Energies and energy corrections for the reaction of benzaldehyde

\begin{tabular}{|c|c|c|c|c|c|c|}
\hline Structure & $\mathbf{E}_{\text {el }}(\mathbf{a . u .})$ & $\mathbf{E}_{\mathbf{b b}}(\mathbf{a . u .})$ & $\mathbf{E}_{\text {solv }}(\mathbf{a . u . )}$ & $\begin{array}{c}\mathbf{Z P E} \\
(\mathbf{a . u .})\end{array}$ & $\mathbf{E}_{\text {tot }}$ (a.u.) & $\begin{array}{c}\mathbf{E}_{\text {rel }} \\
\text { (kcal/mol) }\end{array}$ \\
\hline En(BA)-R1 & -7071.05761 & -7072.94612 & -7071.18478 & 2.73497 & -7070.33833 & 0.0 \\
\hline En(BA)-S1 & -7071.05722 & -7072.94619 & -7071.18266 & 2.73504 & -7070.33659 & 1.1 \\
\hline En(BA)-S2 & -7071.03808 & -7072.93013 & -7071.16802 & 2.73317 & -7070.32690 & 7.2 \\
\hline TS1(BA)-R & -7071.03682 & -7072.92306 & -7071.17154 & 2.73649 & -7070.32130 & 10.7 \\
\hline TS1(BA)-S & -7071.02015 & -7072.90884 & -7071.14457 & 2.73496 & -7070.29830 & 25.1 \\
\hline Int1(BA)-R & -7071.03825 & -7072.92373 & -7071.17450 & 2.73671 & -7070.32327 & 9.4 \\
\hline Int1(BA)-S & -7071.02505 & -7072.91469 & -7071.14922 & 2.73700 & -7070.30186 & 22.9 \\
\hline TS2(BA)-S & -7071.01586 & -7072.90234 & -7071.14205 & 2.73314 & -7070.29539 & 26.9 \\
\hline Int2(BA)-R & -7071.03891 & -7072.92727 & -7071.17726 & 2.73661 & -7070.32902 & 5.8 \\
\hline Int2(BA)-S & -7071.03700 & -7072.92358 & -7071.15969 & 2.73655 & -7070.30972 & 18.0 \\
\hline TS3(BA)-R & -7071.02216 & -7072.91153 & -7071.15584 & 2.73611 & -7070.30909 & 18.3 \\
\hline TS3(BA)-S & -7071.02338 & -7072.91031 & -7071.14387 & 2.73642 & -7070.29439 & 27.6 \\
\hline EP(BA)-R & -7071.04115 & -7072.93138 & -7071.16977 & 2.73531 & -7070.32469 & 8.6 \\
\hline EP(BA)-S & -7071.04422 & -7072.93399 & -7071.16794 & 2.73449 & -7070.32323 & 9.5 \\
\hline
\end{tabular}


Table S2. Energies and energy corrections for the reaction of acetaldehyde

\begin{tabular}{|c|c|c|c|c|c|c|}
\hline Structure & $\mathbf{E}_{\text {el }}(\mathbf{a . u .})$ & $\mathbf{E}_{\text {bb }}(\mathbf{a . u .})$ & $\mathbf{E}_{\text {solv }}(\mathbf{a . u .})$ & $\begin{array}{c}\mathbf{Z P E} \\
(\mathbf{a . u .})\end{array}$ & $\mathbf{E}_{\text {tot }}(\mathbf{a . u .})$ & $\begin{array}{c}\mathbf{E}_{\text {rel }} \\
\text { (kcal/mol) }\end{array}$ \\
\hline En(AA)-S1 & -6879.28161 & -6879.40510 & -6881.12302 & 2.68209 & -6878.56442 & 0.0 \\
\hline En(AA)-S2 & -6879.26958 & -6879.39227 & -6881.11199 & 2.68167 & -6878.55301 & 7.2 \\
\hline En(AA)-R1 & -6879.27541 & -6879.39730 & -6881.11792 & 2.68282 & -6878.55700 & 4.7 \\
\hline En(AA)-R2 & -6879.26133 & -6879.38315 & -6881.10364 & 2.68347 & -6878.54199 & 14.1 \\
\hline Int1(AA)-S & -6879.28120 & -6879.41416 & -6881.12309 & 2.68400 & -6878.57205 & -4.8 \\
\hline Int1(AA)-R & -6879.27358 & -6879.41102 & -6881.11561 & 2.68366 & -6878.56939 & -3.1 \\
\hline Int2(AA)-S & -6879.28126 & -6879.41425 & -6881.12335 & 2.68378 & -6878.57255 & -5.1 \\
\hline Int2(AA)-R & -6879.28271 & -6879.41423 & -6881.12195 & 2.68387 & -6878.56960 & -3.2 \\
\hline TS3(AA)-S & -6879.26296 & -6879.39408 & -6881.10633 & 2.68222 & -6878.55523 & 5.8 \\
\hline TS3(AA)-R & -6879.27066 & -6879.39921 & -6881.11009 & 2.68388 & -6878.55476 & 6.1 \\
\hline EP(AA)-S & -6879.27394 & -6879.40125 & -6881.11836 & 2.68159 & -6878.56407 & 0.2 \\
\hline EP(AA)-R & -6879.28065 & -6879.40748 & -6881.12355 & 2.68316 & -6878.56722 & -1.8 \\
\hline
\end{tabular}

$\mathbf{E}_{\mathbf{e l}}=$ Energy of the optimized geometry at B3LYP-D3(BJ)/6-31G(d,p) level.

$\mathbf{E}_{\mathbf{b b}}=$ Single-point energy of the optimized geometry at B3LYP-D3(BJ)/6-311+G(2d,2p) level.

$\mathbf{E}_{\text {solv }}=$ Single-point energy of the optimized geometry at B3LYP-D3(BJ)/6-31G(d,p) and SMD solvation method with $\varepsilon=4$.

$\mathbf{E}_{\text {tot }}=\mathrm{E}_{\mathrm{bb}}+\left(\mathrm{E}_{\mathrm{solv}}-\mathrm{E}_{\mathrm{el}}\right)+\mathrm{ZPE}$

$\mathbf{E}_{\text {rel }}=$ Energy relative to the lowest-energy binding mode. 


\section{Cartesian coordinates of optimized structures}

\section{Reaction of benzaldehyde}

\section{En(BA)-R1}

\begin{tabular}{|c|c|c|c|}
\hline C & 11.2585 & 5.0081 & -0.2545 \\
\hline$C$ & 9.7663 & 4.7507 & -0.5305 \\
\hline C & 9.1422 & 3.6672 & 0.3065 \\
\hline C & 8.3454 & 2.6181 & -0.1022 \\
\hline$\Lambda$ & 9.2498 & 3.6573 & 1.6864 \\
\hline $\mathrm{C}$ & 8.5273 & 2.6267 & 2.0918 \\
\hline 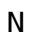 & 7.9610 & 1.9566 & 1.0493 \\
\hline $\mathrm{H}$ & 11.4078 & 5.2269 & 0.8059 \\
\hline $\mathrm{H}$ & 9.6174 & 4.5066 & -1.5898 \\
\hline $\mathrm{H}$ & 9.2176 & 5.6864 & -0.3536 \\
\hline $\mathrm{H}$ & 8.0221 & 2.2939 & -1.0790 \\
\hline $\mathrm{H}$ & 8.3736 & 2.3155 & 3.1156 \\
\hline $\mathrm{H}$ & 7.2493 & 1.2208 & 1.1255 \\
\hline C & -9.4544 & 1.9548 & -4.9747 \\
\hline C & -8.4306 & 2.5980 & -4.0229 \\
\hline C & -7.4722 & 1.6005 & -3.4080 \\
\hline C & -6.5595 & 0.8968 & -4.2084 \\
\hline C & -7.4547 & 1.3448 & -2.0325 \\
\hline C & -5.6726 & -0.0245 & -3.6635 \\
\hline l & -6.5681 & 0.4267 & -1.4641 \\
\hline C & -5.6655 & -0.2642 & -2.2821 \\
\hline 0 & -4.7733 & -1.1775 & -1.8133 \\
\hline $\mathrm{H}$ & -10.0625 & 1.2146 & -4.4444 \\
\hline $\mathrm{H}$ & -8.9597 & 3.1300 & -3.2237 \\
\hline $\mathrm{H}$ & -7.8648 & 3.3591 & -4.5770 \\
\hline $\mathrm{H}$ & -6.5341 & 1.0825 & -5.2796 \\
\hline $\mathrm{H}$ & -8.1230 & 1.8937 & -1.3739 \\
\hline $\mathrm{H}$ & -4.9616 & -0.5565 & -4.2859 \\
\hline $\mathrm{H}$ & -6.5560 & 0.2938 & -0.3882 \\
\hline $\mathrm{H}$ & -4.7572 & -1.2260 & -0.8209 \\
\hline C & -0.4270 & -7.0527 & -4.5497 \\
\hline C & -0.5133 & -6.4084 & -3.1588 \\
\hline C & -1.8886 & -5.9164 & -2.7502 \\
\hline $\mathrm{C}$ & -2.3367 & -6.0826 & -1.4348 \\
\hline C & -2.7384 & -5.2401 & -3.6387 \\
\hline C & -3.5744 & -5.5984 & -1.0137 \\
\hline $\mathrm{C}$ & -3.9782 & -4.7500 & -3.2364 \\
\hline C & -4.4047 & -4.9330 & -1.9186 \\
\hline 0 & -5.6509 & -4.4822 & -1.5811 \\
\hline $\mathrm{H}$ & -0.6723 & -6.3394 & -5.3425 \\
\hline $\mathrm{H}$ & -0.1661 & -7.1272 & -2.4081 \\
\hline $\mathrm{H}$ & 0.1953 & -5.5698 & -3.1107 \\
\hline $\mathrm{H}$ & -1.7059 & -6.6022 & -0.7195 \\
\hline $\mathrm{H}$ & -2.4289 & -5.0850 & -4.6678 \\
\hline $\mathrm{H}$ & -3.8960 & -5.7432 & 0.0148 \\
\hline $\mathrm{H}$ & -4.6223 & -4.2129 & -3.9237 \\
\hline $\mathrm{H}$ & -5.7440 & -4.4644 & -0.6170 \\
\hline C & 4.8619 & -4.7711 & -4.8668 \\
\hline C & 5.0519 & -5.3580 & -3.4905 \\
\hline 0 & 6.0890 & -5.9291 & -3.1521 \\
\hline $\mathrm{C}$ & 6.1449 & -4.1441 & -5.4138 \\
\hline $\mathrm{H}$ & 4.5424 & -5.5902 & -5.5251 \\
\hline $\mathrm{H}$ & 6.9581 & -4.8714 & -5.3680 \\
\hline $\mathrm{H}$ & 6.4366 & -3.2721 & -4.8235 \\
\hline $\mathrm{H}$ & 6.0129 & -3.8216 & -6.4505 \\
\hline $\mathrm{N}$ & 3.9795 & -5.2483 & -2.6740 \\
\hline $\mathrm{C}$ & 4.0083 & -5.8271 & -1.3498 \\
\hline C & 2.6404 & -5.7919 & -0.6727 \\
\hline C & 2.0053 & -4.3982 & -0.5051 \\
\hline $\mathrm{C}$ & 0.7172 & -4.5204 & 0.3134 \\
\hline $\mathrm{C}$ & 2.9594 & -3.3909 & 0.1415 \\
\hline $\mathrm{H}$ & 3.1682 & -4.7259 & -2.9658 \\
\hline $\mathrm{H}$ & 4.7447 & -5.2981 & -0.7364 \\
\hline $\mathrm{H}$ & 2.7625 & -6.2386 & 0.3221 \\
\hline $\mathrm{H}$ & 1.9435 & -6.4407 & -1.2201 \\
\hline $\mathrm{H}$ & 1.7236 & -4.0094 & -1.4975 \\
\hline$\pi$ & 0.9439 & -4.8846 & 1. 3227 \\
\hline $\mathrm{H}$ & 0.2129 & -3.5619 & 0.4273 \\
\hline $\mathrm{H}$ & 0.0087 & -5.2128 & -0 \\
\hline
\end{tabular}

\begin{tabular}{|c|c|c|c|}
\hline & 060 & -3.2974 & -0 \\
\hline & 799 & 777 & \\
\hline & 150 & 3933 & \\
\hline & 746 & -5 & \\
\hline & 260 & -4.8286 & -0.226 \\
\hline & 755 & -3.4695 & -0 \\
\hline & & & -2 \\
\hline & & & \\
\hline & & -1 & -2 \\
\hline & & 801 & -0 \\
\hline & & 9631 & -1 . \\
\hline & & -5.1214 & \\
\hline & & -4 & \\
\hline & & -5 & -0 \\
\hline & & -4 & -2 \\
\hline & & -2 & \\
\hline & 8.2791 & -1 & -3 \\
\hline & 9.0276 & -0 . & \\
\hline & 8. & 667 & -2 \\
\hline & -2. & 17 & \\
\hline & -1 & & -0 \\
\hline & & & -0 \\
\hline & & & -1 \\
\hline & -2 & & -1 \\
\hline & -3.7595 & & -0 \\
\hline & -3.9482 & 55 & 0. \\
\hline & & -0 & -2 \\
\hline & & & -1 \\
\hline & -4. & & -0 \\
\hline & 34 & -0 & -0 \\
\hline & -0.3903 & -1 & -1 \\
\hline & -1.6618 & -2 & -1 \\
\hline & 0 & -1 & -2 \\
\hline & -0 & -2 & -3 \\
\hline & & & \\
\hline & 0 & & -1 \\
\hline & -0.2 & & -1 \\
\hline & -0.9010 & -0 & \\
\hline & 0.2080 & & \\
\hline & -5. & 64 & -0 \\
\hline & -4 . & & -1 \\
\hline & -5 & & -1 \\
\hline & -3 & -0 & \\
\hline & -1.6678 & -2 & \\
\hline & -1.8069 & -3 & -1 \\
\hline & -2.5196 & -1 & -1 \\
\hline & 96 & -3 & -4 \\
\hline & -0 . & -2 & -4 \\
\hline & & -2 & \\
\hline & & -1 & \\
\hline & 1.1973 & -0 & \\
\hline & 634 & -0 & \\
\hline & & & \\
\hline & -8 & & \\
\hline & -7 & & \\
\hline & -6 & & \\
\hline & -7.0615 & & \\
\hline & & & \\
\hline & -9. & & \\
\hline & -8 & & \\
\hline & -8 & & \\
\hline & -7 & & \\
\hline & -1 . & & \\
\hline & -1.0883 & & \\
\hline & & & \\
\hline & & & \\
\hline & 1 & & \\
\hline & 2 & & \\
\hline & & & \\
\hline & -0. & & \\
\hline & -1 & & \\
\hline & -1 & & \\
\hline & -0 & & \\
\hline & & & \\
\hline & & & \\
\hline & & & \\
\hline & 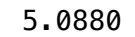 & & \\
\hline & & & \\
\hline & & & \\
\hline & & & \\
\hline & & & \\
\hline
\end{tabular}

\begin{tabular}{|c|c|c|c|}
\hline & .0355 & 7674 & 4.040 \\
\hline & .4949 & 3701 & . 819 \\
\hline & 940 & 9771 & 3180 \\
\hline & & & \\
\hline & 6. & 361 & \\
\hline & 05 & & TU \\
\hline & & & \\
\hline & & & \\
\hline & 5.5792 & 596 & $=0$ \\
\hline & -1.6 & & 2 \\
\hline & & & \\
\hline & 11. & & \\
\hline & 11.6 & & -0 . \\
\hline & -10.1251 & 077 & $-5.4 e$ \\
\hline & & & \\
\hline & -1.3 & & \\
\hline & -2.2973 & & 5. \\
\hline & 6.1943 & & \\
\hline & & & \\
\hline & -0.5 & -1 & \\
\hline & 0.2 & -0. & 8. \\
\hline & & -2 . & \\
\hline & 0. & & \\
\hline & 2.6 & -2 & 8. \\
\hline & 8.5 & -6 & 0. \\
\hline & 9.5 & & \\
\hline & 4.3 & -6 . & -1 \\
\hline & 0.5 & -7 & -4 \\
\hline & -1]$. & -7 & -4 \\
\hline & & -4 . & \\
\hline & & & \\
\hline y & -4.9 & & \\
\hline$C$ & 2.1 & & -3 \\
\hline & & & \\
\hline 0 & 0. & & -2 \\
\hline & -0.1 & 6. & -2 \\
\hline$C$ & -1. & & -2 \\
\hline 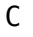 & -1 & & \\
\hline 0 & -1 & & -4 . \\
\hline & -2.7 & 4. & -3 \\
\hline & -3. & & \\
\hline & -3. & & \\
\hline & -1.8 & & -4 \\
\hline & -2.6 & & -4 \\
\hline & -0. & & \\
\hline & 3. & & -1 \\
\hline C & 4.2 & & -0. \\
\hline & 5.6 & & -1 \\
\hline & & & \\
\hline E & 3.7 & & -3 \\
\hline & 2.9 & & -2 \\
\hline$c$ & 2.2 & & -0 . \\
\hline & & & \\
\hline 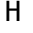 & 2.2 & & -2 \\
\hline 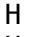 & 2.9 & & -2 \\
\hline H & -1.5 & & -1 \\
\hline & & & \\
\hline & -3 & & \\
\hline & -3.6 & & -5 . \\
\hline & -1.4 & & \\
\hline & & & \\
\hline & -1.2 & & \\
\hline & -0.6 & & -5 . \\
\hline & -2. & & \\
\hline & & & \\
\hline & -1 & & -3 \\
\hline & -2 & & -5 . \\
\hline & & & \\
\hline & & & \\
\hline & 5. & & -3 \\
\hline & & & -4 \\
\hline 1 & & & -3 \\
\hline & & & \\
\hline & & & \\
\hline 0 & & -1 & \\
\hline 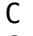 & & & $2.07+3$ \\
\hline & -5.8 & & \\
\hline & & & \\
\hline & & & \\
\hline $\mathbf{v}$ & & & \\
\hline & -1.7959 & -2.8580 & 4.4627 \\
\hline
\end{tabular}




\begin{tabular}{|c|c|c|c|c|c|c|c|c|c|c|c|}
\hline C & -0.9849 & -2.3295 & 3.2700 & $\mathrm{H}$ & 4.2599 & -1.7349 & -4.8370 & $\mathrm{H}$ & 3.3899 & -3.6671 & 1.1885 \\
\hline 0 & -1.3325 & -2.5827 & 2.1086 & $\mathrm{H}$ & 6.3150 & 0.8098 & -2.0731 & H & 2.7647 & -2.3272 & 0.2162 \\
\hline C & -1.1542 & -4.1710 & 4.9522 & $\mathrm{H}$ & 7.2041 & 2.1624 & -3.9688 & C & 8.8952 & -5.6704 & 0.0587 \\
\hline C & -1.7215 & -5.1964 & 3.9571 & C & 4.7671 & -1.3765 & -2.2332 & C & 7.6221 & -4.8619 & -0.243 \\
\hline C & -3.1620 & -4.7137 & 3.6989 & H & 4.2603 & -2.3145 & -2.5267 & C & 7.9469 & -3.4461 & -0.662 \\
\hline $\mathrm{N}$ & 0.1471 & -1.6922 & 3.6044 & 0 & 4.9059 & -1.1104 & -1.0495 & C & 7.8926 & -3.0755 & -2.0122 \\
\hline $\mathrm{C}$ & 1.1712 & -1.3687 & 2.6391 & & & & & $\mathrm{C}$ & 8.3787 & -2.4974 & $\begin{array}{r}0.2742 \\
-2.4148\end{array}$ \\
\hline C & 2.5392 & -1.8468 & 3.1157 & & & & & C & 8.2682 & -1.7932 & \\
\hline 0 & 2.6931 & -2.8840 & 3.7573 & \multirow{2}{*}{\multicolumn{4}{|c|}{$\operatorname{En}(B A)-S 1$}} & C & 8.7628 & -1.2163 & -0.123 \\
\hline $\mathrm{N}$ & 3.5550 & -1.0167 & 2.7562 & & & & & c & 8.7100 & -0.8620 & -1.473 \\
\hline C & 4.9322 & -1.2656 & 3.1261 & & & & & $\mathrm{H}$ & 9.4758 & -5.2061 & 0.862 \\
\hline C & 5.4102 & -0.4303 & 4.3379 & C & 11.3406 & 4.9742 & -0.1253 & $\mathrm{H}$ & 6.9829 & -4.8485 & 0.649 \\
\hline 0 & 6.5953 & -0.0996 & 4.4376 & C & 10.8526 & 3.6186 & -0.6541 & $\mathrm{H}$ & 7.0613 & -5.3598 & $-1.04 e$ \\
\hline C & 5.8820 & -1.1194 & 1.9426 & C & 9.6794 & 3.0124 & 0.0651 & $\mathrm{H}$ & 7.5578 & -3.8073 & -2.74 \\
\hline 0 & 5.7144 & 0.1402 & 1.2760 & C & 8.3873 & 2.8450 & -0.3874 & $\mathrm{H}$ & 8.4219 & -2.7688 & 1.326 \\
\hline $\mathrm{N}$ & 4.4835 & -0.1883 & 5.2889 & $\mathrm{~N}$ & 9.7978 & 2.4798 & 1.3376 & $\mathrm{H}$ & 8.2063 & -1.5165 & -3.463 \\
\hline C & 4.8658 & 0.3234 & 6.5968 & C & 8.6004 & 2.0026 & 1.6407 & $\mathrm{H}$ & 9.1047 & -0.4936 & 0.610 \\
\hline $\mathrm{H}$ & -7.3838 & -2.1931 & 2.7049 & $\mathrm{~N}$ & 7.7107 & 2.1950 & 0.6250 & $\mathrm{H}$ & 8.9994 & 0.1385 & -1.77 \\
\hline $\mathrm{H}$ & -6.1476 & -0.9328 & 2.9757 & $\mathrm{H}$ & 10.5619 & 5.7299 & -0.2215 & C & -2.8183 & 0.8097 & 0.508 \\
\hline $\mathrm{H}$ & -5.4929 & -3.9266 & 3.1148 & H & 11.6836 & 2.9125 & -0.6056 & C & -1.5855 & 1.0549 & -0.044 \\
\hline $\mathrm{H}$ & -1.4884 & -4.3839 & 5.9729 & $\mathrm{H}$ & 10.5993 & 3.7182 & -1.7108 & C & -1.4548 & 2.2949 & -0.756 \\
\hline $\mathrm{H}$ & -0.0636 & -4.1201 & 4.9428 & $\mathrm{H}$ & 7.8990 & 3.1321 & -1.3040 & $\mathrm{~N}$ & -0.3004 & 2.7236 & -1.251 \\
\hline $\mathrm{H}$ & -1.6916 & -6.2228 & 4.3314 & H & 8.3149 & 1.5093 & 2.5611 & $\mathrm{~N}$ & -2.5309 & 3.1049 & -0.947 \\
\hline $\mathrm{H}$ & -1.1528 & -5.1528 & 3.0252 & $\mathrm{H}$ & 6.7464 & 1.8762 & 0.6242 & C & -3.6963 & 2.7808 & -0.414 \\
\hline $\mathrm{H}$ & -3.8849 & -5.2530 & 4.3244 & C & -9.3956 & 2.1384 & -4.8784 & $\mathrm{~N}$ & -3.8605 & 1.6722 & 0.337 \\
\hline $\mathrm{H}$ & -3.4439 & -4.8476 & 2.6491 & C & -8.2629 & 2.5880 & -3.9419 & $\mathrm{~S}$ & 1.2938 & -0.2617 & -3.315 \\
\hline $\mathrm{H}$ & -1.8678 & -2.0922 & 5.2379 & C & -7.4370 & 1.4518 & -3.3727 & C & 1.3241 & 0.1818 & -1.596 \\
\hline $\mathrm{H}$ & 1.2050 & -0.2990 & 2.4481 & C & -6.8543 & 0.4870 & -4.2088 & C & -4.8752 & 3.6507 & -0.693 \\
\hline $\mathrm{H}$ & 0.9335 & -1.8708 & 1.7011 & C & -7.2049 & 1.3380 & -1.9972 & $\mathrm{~N}$ & 0.2542 & -0.4546 & $-0.95 e$ \\
\hline $\mathrm{H}$ & 6.9017 & -1.1782 & 2.3271 & C & -6.0652 & -0.5361 & -3.6968 & C & -0.4457 & -1.3822 & -1.757 \\
\hline $\mathrm{H}$ & 5.7115 & -1.9291 & 1.2303 & C & -6.4002 & 0.3270 & -1.4659 & C & -1.6228 & -2.1285 & -1.200 \\
\hline $\mathrm{H}$ & 5.3970 & -0.0644 & 0.3785 & C & -5.8084 & -0.6101 & -2.3210 & C & -0.0086 & -1.4222 & -3.033 \\
\hline $\mathrm{H}$ & 4.9708 & -2.3089 & 3.4644 & 0 & -4.9842 & -1.6029 & -1.8890 & C & -0.5164 & -2.2153 & $-4.19 l$ \\
\hline $\mathrm{H}$ & 5.6509 & 1.0719 & 6.4769 & $\mathrm{H}$ & -10.0897 & 1.4722 & -4.3558 & C & -0.4153 & 0.1300 & 0.236 \\
\hline $\mathrm{H}$ & 2.1867 & 6.6498 & -4.2141 & $\mathrm{H}$ & -8.6851 & 3.1683 & -3.1132 & $\mathrm{H}$ & 0.5400 & 2.1602 & -1.193 \\
\hline $\mathrm{H}$ & -4.2742 & 4.5721 & -4.9295 & $\mathrm{H}$ & -7.6068 & 3.2777 & -4.4919 & $\mathrm{H}$ & -0.2152 & 3.6066 & -1.762 \\
\hline $\mathrm{H}$ & -2.7327 & 4.1998 & -2.6662 & $\mathrm{H}$ & -7.0112 & 0.5398 & -5.2832 & $\mathrm{H}$ & -0.7713 & -0.6871 & 0.857 \\
\hline $\mathrm{H}$ & 0.0094 & 7.7848 & -2.4295 & $\mathrm{H}$ & -7.6427 & 2.0534 & -1.3060 & $\mathrm{H}$ & 0.3420 & 0.6637 & 0.796 \\
\hline $\mathrm{H}$ & 5.2525 & -0.4779 & 7.2398 & $\mathrm{H}$ & -5.6071 & -1.2730 & -4.3489 & $\mathrm{H}$ & -5.6132 & 3.6141 & 0.112 \\
\hline $\mathrm{H}$ & 3.9859 & 0.7730 & 7.0604 & $\mathrm{H}$ & -6.2342 & 0.2887 & -0.3953 & $\mathrm{H}$ & -4.5419 & 4.6698 & $-0.89 e$ \\
\hline $\mathrm{H}$ & 3.5154 & -0.4841 & 5.1805 & $\mathrm{H}$ & -4.7958 & -1.5186 & -0.9147 & $\mathrm{H}$ & -5.3795 & 3.2706 & $-1.58 \mathrm{~s}$ \\
\hline $\mathrm{H}$ & 0.3918 & -1.4255 & 4.5693 & C & -0.4204 & -6.9282 & -4.6500 & $\mathrm{H}$ & -3.0381 & -0.0749 & 1.099 \\
\hline $\mathrm{H}$ & -7.6482 & -2.9080 & 0.5784 & C & -0.3911 & -6.0353 & -3.4006 & $\mathrm{H}$ & -1.4993 & -2.4053 & -0.163 \\
\hline $\mathrm{H}$ & -6.4815 & -2.5169 & -0.6693 & C & -1.7609 & -5.7063 & -2.8470 & $\mathrm{H}$ & -1.7867 & -3.0417 & -1.781 \\
\hline $\mathrm{H}$ & 3.3611 & -0.2318 & 2.1420 & C & -2.0991 & -6.0133 & -1.5260 & $\mathrm{H}$ & -2.5339 & -1.5295 & -1.297 \\
\hline $\mathrm{H}$ & -6.0529 & -3.0149 & 4.5087 & C & -2.7350 & -5.0700 & -3.6331 & $\mathrm{H}$ & 0.2620 & -2.8602 & -4.615 \\
\hline C & -11.2046 & -4.8904 & 1.6888 & C & -3.3540 & -5.7032 & -1.0006 & $\mathrm{H}$ & -0.8708 & -1.5543 & -4.991 \\
\hline C & -10.2312 & -4.2257 & 0.7376 & C & -3.9894 & -4.7483 & -3.1261 & C & 1.1670 & -2.4904 & $7.74 \mathrm{~S}$ \\
\hline 0 & -9.1188 & -3.8567 & 1.1251 & C & -4.3060 & -5.0753 & -1.8047 & C & 0.4833 & -1.1227 & 7.675 \\
\hline C & -11.6766 & -3.8503 & 2.7104 & 0 & -5.5712 & -4.7916 & -1.3655 & C & 1.2826 & -0.1235 & 6.831 \\
\hline $\mathrm{N}$ & -10.6777 & -3.9990 & -0.5174 & $\mathrm{H}$ & -0.9624 & -6.4491 & -5.4717 & 0 & 1.5433 & -0.5104 & 5.619 \\
\hline C & -9.8926 & -3.2544 & -1.4855 & H & 0.1960 & -6.5298 & -2.6204 & 0 & 1.6201 & 0.9672 & 7.310 \\
\hline C & -9.1828 & 7.2733 & 2.3190 & $\mathrm{H}$ & 0.1452 & -5.1068 & -3.6345 & C & -8.6585 & 3.0966 & 3.616 \\
\hline C & -9.0451 & 5.9611 & 1.5551 & H & -1.3704 & -6.5099 & -0.8926 & C & -7.7566 & 1.9189 & 3.232 \\
\hline C & -8.8557 & 6.1395 & 0.0504 & $\mathrm{H}$ & -2.5059 & -4.8131 & -4.6635 & C & -6.9464 & 2.1207 & 1.94 \\
\hline $\mathrm{N}$ & -8.3540 & 5.0314 & -0.5691 & $\mathrm{H}$ & -3.5927 & -5.9568 & 0.0297 & 0 & -7.2588 & 3.0640 & 1.17 \\
\hline 0 & -9.1683 & 7.1637 & -0.5499 & $\mathrm{H}$ & -4.7289 & -4.2310 & -3.7262 & 0 & -6.0048 & 1.2792 & 1.746 \\
\hline H & -10.8159 & -3.4191 & 3.2265 & $\mathrm{H}$ & -5.6045 & -4.8611 & -0.4013 & H & -9.2395 & 2.8645 & 4.515 \\
\hline $\mathrm{H}$ & -12.3387 & -4.3020 & 3.4542 & C & 4.8812 & -4.6714 & -4.9278 & $\mathrm{H}$ & -8.0673 & 3.9932 & $3.81 \mathrm{~s}$ \\
\hline $\mathrm{H}$ & -12.2209 & -3.0373 & 2.2197 & C & 5.0181 & -5.3828 & -3.5982 & $\mathrm{H}$ & -8.3549 & 1.0082 & 3.09 \\
\hline $\mathrm{H}$ & -12.0546 & -5.3284 & 1.1536 & 0 & 5.9908 & -6.0923 & -3.3338 & $\mathrm{H}$ & -7.0458 & 1.6825 & $4.03]$ \\
\hline $\mathrm{H}$ & -9.6408 & -2.2600 & -1.1044 & C & 6.1569 & -3.9163 & -5.3092 & C & -1.2639 & 2.2313 & 5.443 \\
\hline $\mathrm{H}$ & -8.2219 & 5.3538 & 1.9376 & $\mathrm{H}$ & 4.6721 & -5.4445 & -5.6783 & C & -1.0489 & 1.6342 & 4.046 \\
\hline $\mathrm{H}$ & -9.9490 & 5.3528 & 1.6905 & $\mathrm{H}$ & 7.0191 & -4.5840 & -5.2400 & C & 0.2675 & 2.0187 & 3.45 \\
\hline $\mathrm{H}$ & -7.9234 & 4.2888 & -0.0197 & $\mathrm{H}$ & 6.3205 & -3.0699 & -4.6365 & C & 0.6031 & 2.7309 & 2.321 \\
\hline $\mathrm{H}$ & -8.1215 & 5.1219 & -1.5466 & $\mathrm{H}$ & 6.0945 & -3.5256 & -6.3294 & $\mathrm{~N}$ & 1.4730 & 1.6593 & 4.024 \\
\hline $\mathrm{H}$ & -9.9777 & 7.8876 & 1.8886 & $\mathrm{~N}$ & 3.9637 & -5.2198 & -2.7581 & C & 2.4627 & 2.1262 & 3.230 \\
\hline $\mathrm{H}$ & -9.4090 & 7.0855 & 3.3732 & C & 4.0260 & -5.7941 & -1.4320 & $\mathrm{~N}$ & 1.9799 & 2.7817 & 2.17 \\
\hline $\mathrm{H}$ & -8.2582 & 7.8565 & 2.2678 & C & 2.6964 & -5.7053 & -0.6845 & $\mathrm{H}$ & -0.4675 & 1.9337 & 6.131 \\
\hline $\mathrm{H}$ & -10.6733 & -5.7020 & 2.1944 & C & 2.1344 & -4.2872 & -0.4762 & $\mathrm{H}$ & -1.1271 & 0.5422 & 4.099 \\
\hline $\mathrm{H}$ & -10.4700 & -3.1451 & -2.4039 & C & 0.8640 & -4.3516 & 0.3747 & $\mathrm{H}$ & -1.8413 & 1.9635 & 3.366 \\
\hline $\mathrm{H}$ & -8.9580 & -3.7759 & -1.7171 & C & 3.1581 & -3.3472 & 0.1679 & $\mathrm{H}$ & -0.0560 & 3.1978 & 1.604 \\
\hline $\mathrm{H}$ & -11.6018 & -4.3115 & -0.7684 & $\mathrm{H}$ & 3.4285 & -4.3675 & -2.8668 & $\mathrm{H}$ & 3.5090 & 1.9660 & 3.442 \\
\hline C & 6.2373 & 0.9556 & -5.4726 & $\mathrm{H}$ & 4.8139 & -5.3109 & -0.8401 & $\mathrm{H}$ & 2.3684 & 1.7083 & 0.843 \\
\hline C & 5.4042 & -0.1370 & -5.7242 & $\mathrm{H}$ & 2.8472 & -6.1671 & 0.3007 & C & 5.2538 & 7.0992 & 3.020 \\
\hline C & 4. 9163 & -0.8886 & -4.6596 & $\mathrm{H}$ & 1.9456 & -6.3168 & -1.2013 & C & 5.0970 & 5.9718 & 4.045 \\
\hline C & 5.2541 & -0.5469 & -3.3445 & $\mathrm{H}$ & 1.8624 & -3.8665 & -1.4542 & C & 5.7658 & 4.6389 & 3.661 \\
\hline C & 6.0786 & 0.5568 & -3.0974 & $\mathrm{H}$ & 1.1003 & -4.6867 & 1. 3919 & C & 5.5743 & 3.6028 & 4.777 \\
\hline C & 6.5713 & 1.3023 & -4.1600 & $\mathrm{H}$ & 0.3881 & -3.3755 & 0.4616 & C & 5.2419 & 4.1032 & 2.320 \\
\hline $\mathrm{H}$ & 6.6222 & 1.5431 & -6.3009 & $\mathrm{H}$ & 0.1199 & -5.0329 & -0.0481 & H & 4.7757 & 6.8456 & 2.065 \\
\hline & 5.1383 & -0.3978 & -6.7437 & $\mathrm{H}$ & 4.0855 & -3.3149 & -0.4036 & $\mathrm{H}$ & 4.0276 & 5.7836 & 4.214 \\
\hline
\end{tabular}




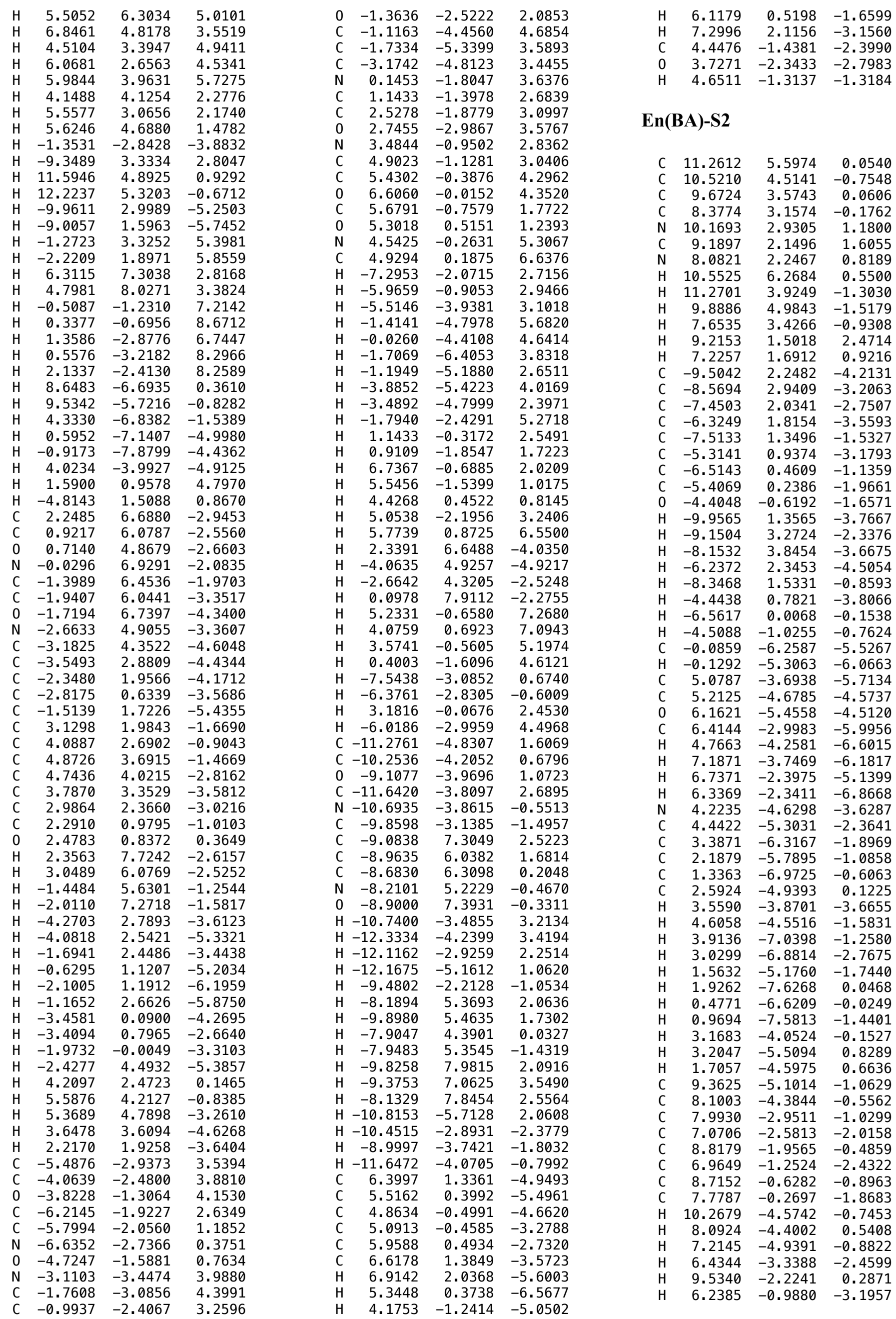




\begin{tabular}{|c|c|c|c|}
\hline $\mathrm{H}$ & 9.3460 & 0.1315 & -0.4471 \\
\hline $\mathrm{H}$ & 7.6892 & 0.7681 & -2.1740 \\
\hline C & -2.8795 & 0.8481 & 0.9420 \\
\hline$C$ & -1.6170 & 1. 2262 & 0.5751 \\
\hline $\mathrm{C}$ & -1.4878 & 2.5676 & 0.0804 \\
\hline $\mathrm{N}$ & -0.3106 & 3.1673 & -0.0342 \\
\hline $\mathrm{N}$ & -2.5896 & 3.2783 & -0.2859 \\
\hline C & -3.7859 & 2.8162 & 0.0449 \\
\hline $\mathrm{N}$ & -3.9457 & 1.6641 & 0.7291 \\
\hline $\mathrm{S}$ & 0.0162 & 0.9370 & -3.2158 \\
\hline$C$ & 0.7093 & 0.8089 & -1.6032 \\
\hline$C$ & -5.0042 & 3.5838 & -0.3438 \\
\hline $\mathrm{N}$ & -0.0617 & -0.0689 & -0.8319 \\
\hline C & -1.0404 & -0.7647 & -1.5871 \\
\hline C & -1.7285 & -1.9295 & -0.9639 \\
\hline$C$ & -1.1520 & -0.3469 & -2.8616 \\
\hline C & -2.0313 & -0.8470 & -3.9599 \\
\hline C & -0.4513 & 0.2686 & 0.5732 \\
\hline $\mathrm{H}$ & 0.5214 & 2.7476 & 0.3570 \\
\hline $\mathrm{H}$ & -0.1859 & 4.0394 & -0.5538 \\
\hline H & -0.7306 & -0.6590 & 1.0705 \\
\hline $\mathrm{H}$ & 0.4144 & 0.6765 & 1.0791 \\
\hline $\mathrm{H}$ & -5.7529 & 3.5087 & 0.4492 \\
\hline $\mathrm{H}$ & -4.7396 & 4.6217 & -0.5443 \\
\hline $\mathrm{H}$ & -5.4341 & 3.1466 & -1.2504 \\
\hline $\mathrm{H}$ & -3.1102 & -0.1032 & 1.4120 \\
\hline $\mathrm{H}$ & -0.9949 & -2.6267 & -0.5518 \\
\hline $\mathrm{H}$ & -2.3301 & -2.4428 & -1.7086 \\
\hline $\mathrm{H}$ & -2.3864 & -1.6588 & -0.1389 \\
\hline $\mathrm{H}$ & -2.8854 & -1.3803 & -3.5434 \\
\hline $\mathrm{H}$ & -1.4924 & -1.5130 & -4.6426 \\
\hline C & 1.8479 & -3.3733 & 7.2758 \\
\hline C & 1.0008 & -2.0991 & 7.1812 \\
\hline$C$ & 1.7656 & -0.9876 & 6.4518 \\
\hline 0 & 1.9979 & -1.2143 & 5.1924 \\
\hline 0 & 2.1384 & 0.0139 & 7.0735 \\
\hline C & -8.4250 & 2.1442 & 4. 2981 \\
\hline$C$ & -7.5771 & 1.0590 & 3.6468 \\
\hline C & -6.7745 & 1.5430 & 2.4371 \\
\hline 0 & -6.9312 & 2.7153 & 2.0239 \\
\hline 0 & -5.9669 & 0.6868 & 1.9396 \\
\hline $\mathrm{H}$ & -8.9501 & 1.7565 & 5.1786 \\
\hline $\mathrm{H}$ & -7.8051 & 2.9859 & 4.6147 \\
\hline $\mathrm{H}$ & -8.1970 & 0.2150 & 3.3159 \\
\hline $\mathrm{H}$ & -6.8586 & 0.6336 & 4.3572 \\
\hline C & -0.9201 & 1.4636 & 5.7051 \\
\hline$C$ & -0.6839 & 1.1378 & 4.2201 \\
\hline C & 0.5795 & 1.7302 & 3.6775 \\
\hline$C$ & 0.8297 & 2.8925 & 2.9735 \\
\hline $\mathrm{N}$ & 1.8205 & 1.1586 & 3.8678 \\
\hline$C$ & 2.7414 & 1.9738 & 3.2825 \\
\hline $\mathrm{N}$ & 2.1794 & 3.0353 & 2.7231 \\
\hline $\mathrm{H}$ & -0.0801 & 1.1276 & 6.3196 \\
\hline $\mathrm{H}$ & -0.6745 & 0.0511 & 4.0780 \\
\hline $\mathrm{H}$ & -1.5233 & 1.5130 & 3.6249 \\
\hline $\mathrm{H}$ & 0.1122 & 3.6325 & 2.6475 \\
\hline $\mathrm{H}$ & 3.7996 & 1.7560 & 3.2920 \\
\hline $\mathrm{H}$ & 2.9643 & 0.6096 & 0.1700 \\
\hline C & 5.2272 & 6.9577 & 3.6877 \\
\hline C & 5.3857 & 5.5414 & 4.2485 \\
\hline C & 6.0607 & 4.5360 & 3. 2971 \\
\hline$C$ & 6.2438 & 3.1882 & 4.0081 \\
\hline C & 5.2685 & 4.3785 & 1.9914 \\
\hline $\mathrm{H}$ & 4.5557 & 6.9752 & 2.8239 \\
\hline $\mathrm{H}$ & 4.3972 & 5.1464 & 4.5186 \\
\hline $\mathrm{H}$ & 5.9655 & 5.5866 & 5.1804 \\
\hline $\mathrm{H}$ & 7.0605 & 4.9204 & 3.0439 \\
\hline $\mathrm{H}$ & 5.2841 & 2.8091 & 4.3734 \\
\hline $\mathrm{H}$ & 6.6710 & 2.4298 & 3.3477 \\
\hline $\mathrm{H}$ & 6.9061 & 3.2852 & 4.8755 \\
\hline $\mathrm{H}$ & 4.2212 & 4.1253 & 2.1903 \\
\hline $\mathrm{H}$ & 5.7038 & 3.5904 & 1.3709 \\
\hline $\mathrm{H}$ & 5.2833 & 5.3041 & 1.4049 \\
\hline $\mathrm{H}$ & -2.4155 & -0.0098 & -4.5538 \\
\hline $\mathrm{H}$ & -9.1687 & 2.5288 & 3.5991 \\
\hline $\mathrm{H}$ & 11.8739 & 5.1304 & 0.8292 \\
\hline $\mathrm{H}$ & 11.9079 & 6.1952 & -0.5966 \\
\hline H & -10.3095 & 2.9162 & -4.5371 \\
\hline $\mathrm{H}$ & -8.9485 & 1.9274 & -5.1002 \\
\hline $\mathrm{H}$ & -1.0257 & 2.5439 & 5.8463 \\
\hline $\mathrm{H}$ & -1 & & \\
\hline
\end{tabular}

\begin{tabular}{|c|c|c|c|}
\hline & 20 & 669 & \\
\hline & 17 & 65 & \\
\hline & 750 & 127 & \\
\hline & 271 & 370 & \\
\hline & 2.1516 & -3.6986 & \\
\hline & & 899 & \\
\hline & 579 & -3 & \\
\hline & & & \\
\hline & 554 & -5.1457 & \\
\hline & 877 & -5 & \\
\hline & 0.8553 & -6.7429 & \\
\hline & -0.9116 & -6.8812 & \\
\hline & 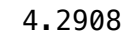 & -2 & \\
\hline & 32 & & \\
\hline & -4.5 & & \\
\hline & 1.5 & & \\
\hline & 0.6988 & 6.4804 & -1 \\
\hline & 0.6411 & 5.2553 & \\
\hline & -0.3480 & 7. & \\
\hline & -1.6 & & -1 \\
\hline & -2. & & -2 \\
\hline & -2 & & \\
\hline & -2.8 & & \\
\hline & -3.4 & 4.7506 & \\
\hline & -2.4686 & 3.8406 & -4 \\
\hline & -1 & 4. & -5 \\
\hline & & & \\
\hline & -1 & & \\
\hline & & & \\
\hline & 3.5 & & \\
\hline & 491 & 4. & -1 \\
\hline & 4.7 & & -2 \\
\hline & & & \\
\hline & & & \\
\hline & & & \\
\hline & 2.1 & 1. & \\
\hline & 2.7716 & 6. & \\
\hline & 2.2514 & & -3 \\
\hline & -1.5 & & -0 \\
\hline & -2.2 & & -0 \\
\hline & -2. & & -4 \\
\hline & -3.6 & & \\
\hline & -0.5491 & & -4 \\
\hline & -0.2439 & & -6 \\
\hline & -1.8 & & -6 \\
\hline & -1 & & -5 \\
\hline & -0. & & -6 \\
\hline & -0.2 & & \\
\hline & 0.7101 & & \\
\hline & -3.6740 & & -4 \\
\hline & 3.5 & & \\
\hline & 80 & & -0 \\
\hline & 4. & & -3 \\
\hline & 3. & & -4 \\
\hline & & & -3 \\
\hline & -4.9631 & -3 & \\
\hline & -3.5410 & -3 & \\
\hline & -3 & -1 & \\
\hline & -5.8 & & \\
\hline & -5 & -2 & \\
\hline & -6 & -2 & \\
\hline & -4 & -1 & \\
\hline & -2. & -3 & \\
\hline & -1 & -3 & \\
\hline & -0 . & & \\
\hline & -0. & -3 & \\
\hline 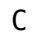 & -0. & -4 & \\
\hline & -1 & & \\
\hline & -2. & & \\
\hline & & -2 & \\
\hline & 1. & -1 & \\
\hline C & & -2 . & \\
\hline & & -3 & \\
\hline & 951 & -1 . & \\
\hline & 5.4142 & -1.4 & \\
\hline & & -0 . & \\
\hline & L21 & -0 . & \\
\hline & 276 & -0 . & \\
\hline & 076 & & \\
\hline & & -0 . & \\
\hline & & & \\
\hline
\end{tabular}

\begin{tabular}{|c|c|c|c|}
\hline 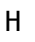 & -6.9209 & -2 & \\
\hline & 433 & -1 & \\
\hline & -4 . & ח77 & \\
\hline & -0.8260 & -5 & \\
\hline & 0.5687 & -4 & \\
\hline & 032 & & \\
\hline & -0 . & & \\
\hline & -3 & & \\
\hline & -2 . & & \\
\hline & 183 & & \\
\hline & 1.6508 & & \\
\hline & 1.4325 & -2.2 & \\
\hline & & -0 . & \\
\hline & & -1 & \\
\hline & & & \\
\hline & 5.5 & & \\
\hline & & & \\
\hline & 1.8936 & & \\
\hline & -4.3 & 4. & \\
\hline & -2 . & & \\
\hline & -0 . & & \\
\hline & & -1 & \\
\hline & & & \\
\hline & 4. & -1 & \\
\hline & 0.9 & -1 & \\
\hline 1 & -7 . & -3.2 & \\
\hline & -6 & -2 . & \\
\hline & & & \\
\hline 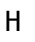 & -5 . & & \\
\hline C & -10. & & \\
\hline 5 & -9 & -4 & \\
\hline D & -8 & -4 & \\
\hline C & -11. & -4 & \\
\hline & -10. & -4 & \\
\hline C & -9 . & -3 & \\
\hline C & -9 . & & \\
\hline t. & -9 . & & \\
\hline & & & \\
\hline & -8 . & & \\
\hline J & -9. & & \\
\hline 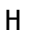 & -10. & -4 & \\
\hline & -11. & -5 & \\
\hline 1 & -11 . & -3 & \\
\hline t & -11.5836 & -5 & \\
\hline 1 & -9.4201 & -2 & \\
\hline | & -8.1338 & & \\
\hline & -9 & & \\
\hline & -7 . & & \\
\hline 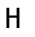 & -8 . & & \\
\hline t & -9 . & & \\
\hline 1 & -9 . & & \\
\hline $\mathrm{H}$ & -8.2 & & \\
\hline $\mathrm{H}$ & -10.1452 & -6 . & \\
\hline 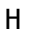 & -10. & -3 & \\
\hline - & -8 & -3 & \\
\hline 1 & -11. & & \\
\hline : & 1. & -2 & \\
\hline C & 1.1 & -2 & \\
\hline C & & -2 & \\
\hline C & 2. & -1 & \\
\hline C & 2. & -0 . & \\
\hline$C$ & 1. & -1 & -4 \\
\hline 1 & & -3 & \\
\hline - & 0. & -3 & \\
\hline H & 2. & -2 & \\
\hline 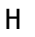 & 3. & & \\
\hline$y$ & 1. & -1 & \\
\hline C & & & \\
\hline 0 & 3.5 & & \\
\hline $\mathrm{H}$ & & & \\
\hline C & -0.1 & -6 . & \\
\hline C & -1. & -5 & \\
\hline$c$ & -1 & & \\
\hline & & & \\
\hline C & -2 . & & \\
\hline 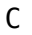 & -3.5 & -4 & \\
\hline 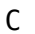 & -3.8 & & \\
\hline 0 & -5. & 656 & \\
\hline & & & \\
\hline & & & \\
\hline & & & \\
\hline
\end{tabular}




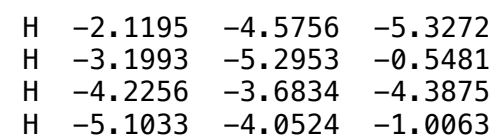

\section{TS1(BA)-R}

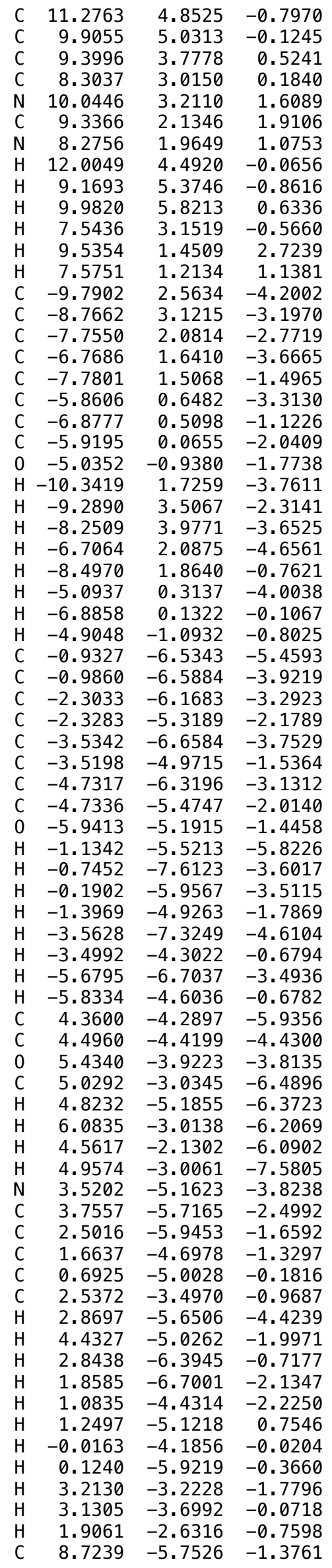

\begin{tabular}{|c|c|c|c|c|c|c|c|}
\hline C & 7.6851 & -4.7123 & -0.9272 & $\mathrm{H}$ & 6.4876 & 2.0734 & 3.27 \\
\hline & 8.3392 & -3.4276 & -0.4720 & H & 6.9201 & 3.1094 & 4.64 \\
\hline & 8.2497 & -2.2621 & -1.2407 & $\mathrm{H}$ & 4.2327 & 3.9358 & \\
\hline & 9.0767 & -3.3898 & 0.7189 & $\mathrm{H}$ & 5.5715 & 3.0052 & \\
\hline & 8.8849 & -1.0882 & -0.8335 & $\mathrm{H}$ & 5.4798 & 4.7405 & 0.91 \\
\hline & 9.7198 & -2.2215 & 1.1249 & $\mathrm{H}$ & -1.2136 & -3.2920 & -3.68 \\
\hline & 9.6304 & -1.0670 & 0.3452 & $\mathrm{H}$ & -9.1666 & 3.1045 & \\
\hline & 9.4154 & -5.9945 & -0.5628 & $\mathrm{H}$ & 11.2194 & 4.1127 & -1.6 \\
\hline & 7.0873 & -5.1327 & -0.1076 & $\mathrm{H}$ & 11.6418 & 5.7952 & -1.21 \\
\hline & 6.9953 & -4.5013 & -1.7514 & $\mathrm{H}$ & -10.5120 & 3.3302 & -4.5 \\
\hline & 7.6614 & -2.2694 & -2.1525 & $\mathrm{H}$ & -9.2912 & 2.1941 & -5.10 \\
\hline & 9.1342 & -4.2793 & 1.3415 & $\mathrm{H}$ & -0.9150 & 2.7740 & 5.5 \\
\hline $\mathrm{H}$ & 8.7804 & -0.1864 & -1.4268 & $\mathrm{H}$ & -1.8288 & 1.2786 & 5 \\
\hline 11 & 10.2697 & -2.2052 & 2.0608 & $\mathrm{H}$ & 6.4319 & 7.0126 & \\
\hline $\mathrm{H}$ & 10.1167 & -0.1520 & 0.6635 & $\mathrm{H}$ & 5.1373 & 7.5316 & 3 \\
\hline$\sigma$ & -2.9840 & 0.8453 & 0.5692 & $\mathrm{H}$ & -0.0975 & -2.0759 & \\
\hline C & -1.7618 & 1.1610 & 0.0308 & $\mathrm{H}$ & 0.6409 & -1.5295 & 8. \\
\hline$C$ & -1.6481 & 2.4758 & -0.5373 & $\mathrm{H}$ & 1.8830 & -3.5833 & 0.1 \\
\hline N & -0.4805 & 3.0149 & -0.8423 & $\mathrm{H}$ & 1.0543 & -4.0137 & \\
\hline N & -2.7686 & 3.2110 & -0.7877 & $\mathrm{H}$ & 2.5714 & -3.0984 & \\
\hline C & -3.9296 & 2.8032 & -0.3069 & $\mathrm{H}$ & 8.2427 & -6.6824 & -1.7 \\
\hline IV & -4.0516 & 1.6791 & 0.4384 & $\mathrm{H}$ & 9.3173 & -5.3666 & -2.2 \\
\hline S & 1.4749 & -0.7637 & -3.1586 & $\mathrm{H}$ & 4.2999 & -6.6684 & -2 . \\
\hline C & 1.1568 & 0.1786 & -1.7426 & $\mathrm{H}$ & 0.0542 & -6.8397 & -5.82 \\
\hline C & -5.1546 & 3.5973 & -0.6056 & $\mathrm{H}$ & -1.6700 & -7.2015 & \\
\hline IV & -0.0047 & -0.2340 & -1.1670 & $\mathrm{H}$ & 3.3003 & -4.3253 & -6.2 \\
\hline 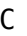 & -0.6771 & -1.2590 & -1.8730 & $\mathrm{H}$ & 1.8454 & 0.4438 & 4.2 \\
\hline C & -2.0217 & -1.7304 & -1.4420 & $\mathrm{H}$ & -4.9885 & 1.4482 & 1.0 \\
\hline C & -0.0038 & -1.6659 & -2.9793 & C & 2.0198 & 6.8601 & \\
\hline C & -0.3819 & -2.6728 & -4.0195 & C & 0.6758 & 6.3286 & -2.32 \\
\hline$C$ & -0.5870 & 0.2247 & 0.1506 & 0 & 0.4306 & 5.1208 & -2.3 \\
\hline $\mathrm{H}$ & 0.3939 & 2.6217 & -0.4817 & $\mathrm{~N}$ & -0.2322 & 7.2356 & -1.87 \\
\hline 11 & -0.4175 & 3.9163 & -1.3177 & C & -1.6063 & 6.8042 & \\
\hline 11 & -0.8914 & -0.6862 & 0.6651 & C & -2.2292 & 6.4038 & -3.0 \\
\hline $\mathrm{H}$ & 0.2185 & 0.6897 & 0.7051 & 0 & -2.0075 & 7.0789 & -4.0 \\
\hline $\mathrm{H}$ & -5.8516 & 3.5511 & 0.2366 & $\mathrm{~N}$ & -3.0170 & 5.3058 & -2.98 \\
\hline 11 & -4.8801 & 4.6278 & -0.8324 & C & -3.5479 & 4.7111 & \\
\hline H & -5.6511 & 3.1664 & -1.4811 & C & -3.1007 & 3.2523 & -4.3 \\
\hline $\mathrm{H}$ & -3.1751 & -0.0746 & 1.1137 & C & -1.5830 & 3.0312 & -4.27 \\
\hline $\mathrm{H}$ & -2.0243 & -2.0883 & -0.4104 & C & -1.2621 & 1.5348 & -4 \\
\hline $\mathrm{H}$ & -2.3451 & -2.5452 & -2.0862 & C & -0.8012 & 3.7729 & -5 \\
\hline $\mathrm{H}$ & -2.7615 & -0.9362 & -1.5471 & C & 2.7337 & 2.1848 & -2.1 \\
\hline $\mathrm{H}$ & 0.4595 & -3.3373 & -4.2431 & C & 3.2874 & 3.3081 & -1.5 \\
\hline$\pi$ & -0.6807 & -2.1820 & -4.9527 & C & 4.0417 & 4.2339 & \\
\hline C & 1.6324 & -3.2340 & 7.1300 & C & 4.2293 & 4.0924 & -3 \\
\hline C & 0.8584 & -1.9128 & 7.0541 & C & 3.6416 & 3.0109 & -4.2 \\
\hline C & 1.6544 & -0.8473 & 6.2889 & C & 2.9105 & 2.0635 & -3.5 \\
\hline 0 & 1.8208 & -1.0865 & 5.0221 & C & 2.1403 & 1.1410 & \\
\hline 0 & 2.0986 & 0.1385 & 6.8880 & 0 & 1.7519 & 1.6905 & -0.0 \\
\hline C & -8.4073 & 2.7527 & 4.2654 & $\mathrm{H}$ & 1.9973 & 7.9246 & -3.00 \\
\hline C & -7.5905 & 1.6097 & 3.6568 & $\mathrm{H}$ & 2.7347 & 6.7010 & -1.94 \\
\hline C & -6.8264 & 1.9733 & 2.3769 & $\mathrm{H}$ & -1.6371 & 5.9854 & -0. \\
\hline 0 & -6.9697 & 3.1165 & 1.8856 & $\mathrm{H}$ & -2.1726 & 7.6414 & -1.2 \\
\hline 0 & -6.0709 & 1.0524 & 1.9062 & $\mathrm{H}$ & -3.5939 & 2.6446 & -3.59 \\
\hline $\mathrm{H}$ & -8.9090 & 2.4237 & 5.1821 & $\mathrm{H}$ & -3.4767 & 2.8846 & -5.32 \\
\hline $\mathrm{H}$ & -7.7665 & 3.6016 & 4.5130 & $\mathrm{H}$ & -1.2423 & 3.4350 & \\
\hline $\mathrm{H}$ & -8.2327 & 0.7510 & 3.4200 & $\mathrm{H}$ & 0.2728 & 3.6021 & -5 . \\
\hline $\mathrm{H}$ & -6.8494 & 1.2295 & 4. 3695 & $\mathrm{H}$ & -1.0889 & 3.4261 & -6.3 \\
\hline C & -0.9075 & 1.6882 & 5.4437 & $\mathrm{H}$ & -0.9683 & 4.8521 & \\
\hline C & -0.8026 & 1.3434 & 3.9491 & $\mathrm{H}$ & -1.5783 & 1.0594 & \\
\hline C & 0.4758 & 1.8464 & 3.3624 & $\mathrm{H}$ & -1.7692 & 1.0169 & -3 \\
\hline C & 0.7772 & 2.9423 & 2.5805 & $\mathrm{H}$ & -0.1869 & 1.3748 & -4.1 \\
\hline N & 1.6993 & 1.2906 & 3.6826 & $\mathrm{H}$ & -3.2037 & 5.3343 & $-5 . e$ \\
\hline C & 2.6602 & 2.0495 & 3.0956 & $\mathrm{H}$ & 3.1513 & 3.4364 & -0. \\
\hline N & 2.1431 & 3.0629 & 2.4142 & $\mathrm{H}$ & 4.4964 & 5.0634 & -1.7 \\
\hline $\mathrm{H}$ & -0.0563 & 1.2899 & 6.0022 & $\mathrm{H}$ & 4.8273 & 4.8103 & -4.1 \\
\hline $\mathrm{H}$ & -0.8866 & 0.2591 & 3.8139 & $\mathrm{H}$ & 3.7670 & 2.8842 & \\
\hline $\mathrm{H}$ & -1.6411 & 1.7882 & 3.4015 & $\mathrm{H}$ & 2.4975 & 1.2277 & -4.1 \\
\hline $\mathrm{H}$ & 0.0878 & 3.6457 & 2.1330 & C & -5.3237 & -3.2667 & 3.4 \\
\hline $\mathrm{H}$ & 3.7092 & 1.8081 & 3.1675 & C & -3.8912 & -2.7266 & \\
\hline $\mathrm{H}$ & 2.5599 & 1.5584 & 0.5608 & 0 & -3.7040 & -1.5457 & 3.88 \\
\hline C & 5.4684 & 6.7192 & 2.9774 & C & -6.1730 & -2.2933 & \\
\hline C & 5.5866 & 5.4008 & 3.7458 & C & -5.7523 & -2.2335 & \\
\hline C & 6.1381 & 4.2130 & 2.9346 & $\mathrm{~N}$ & -6.5784 & -2.8155 & 0.20 \\
\hline C & 6.1985 & 2.9687 & 3.8336 & 0 & -4.6973 & -1.6892 & 0.75 \\
\hline C & 5.3046 & 3.9616 & 1.6685 & $\mathrm{~N}$ & -2.8626 & -3.6131 & \\
\hline $\mathrm{H}$ & 4.7456 & 6.6404 & 2.1596 & C & -1.4970 & -3.2030 & \\
\hline $\mathrm{H}$ & 4.5951 & 5.1208 & 4.1270 & C & -0.7708 & -2.5789 & \\
\hline n & 6.2247 & 5.5512 & 4.6270 & 0 & -1.1941 & -2.7239 & \\
\hline $\mathrm{H}$ & 7.1654 & 4.4487 & 2.6214 & C & -0.8119 & -4.5413 & \\
\hline & 5.2251 & 2.7752 & 4.2970 & C & -1.4784 & -5.5103 & \\
\hline
\end{tabular}




\begin{tabular}{|c|c|c|c|}
\hline $\mathrm{C}$ & -2.9375 & -5.0247 & 3.1036 \\
\hline $\mathrm{N}$ & 0.3794 & -1.9688 & 2.9421 \\
\hline $\mathrm{C}$ & 1.3961 & -1.6423 & 1.9670 \\
\hline C & 2.7164 & -2.3479 & 2.3111 \\
\hline 0 & 2.7532 & -3.5421 & 2.6103 \\
\hline $\mathrm{N}$ & 3.7903 & -1.5321 & 2.2180 \\
\hline C & 5.1651 & -1.9415 & 2.4524 \\
\hline $\mathrm{C}$ & 5.7072 & -1.5546 & 3.8419 \\
\hline 0 & 6.8923 & -1.7617 & 4.1038 \\
\hline C & 6.1207 & -1.3368 & 1.4095 \\
\hline 0 & 6.2126 & 0.0809 & 1.5197 \\
\hline $\mathrm{N}$ & 4.8520 & -0.9461 & 4.6887 \\
\hline $\mathrm{C}$ & 5.3328 & -0.4871 & 5.9790 \\
\hline $\mathrm{H}$ & -7.2267 & -2.5744 & 2.6390 \\
\hline $\mathrm{H}$ & -6.0372 & -1.2764 & 2.9535 \\
\hline $\mathrm{H}$ & -5.3438 & -4.2697 & 2.9798 \\
\hline $\mathrm{H}$ & -1.0422 & -4.8128 & 5.2047 \\
\hline $\mathrm{H}$ & 0.2723 & -4.4876 & 4.0517 \\
\hline $\mathrm{H}$ & -1.4101 & -6.5563 & 3.4891 \\
\hline $\mathrm{H}$ & -1.0070 & -5.4133 & 2.1995 \\
\hline $\mathrm{H}$ & -3.5808 & -5.5877 & 3.7917 \\
\hline $\mathrm{H}$ & -3.3428 & -5.1285 & 2.0916 \\
\hline $\mathrm{H}$ & -1.5089 & -2.4937 & 4.6543 \\
\hline $\mathrm{H}$ & 1.5478 & -0.5626 & 1.9109 \\
\hline $\mathrm{H}$ & 1.0536 & -2.0035 & 0.9969 \\
\hline $\mathrm{H}$ & 7.1141 & -1.7344 & 1.6011 \\
\hline $\mathrm{H}$ & 5.8083 & -1.6317 & 0.4005 \\
\hline $\mathrm{H}$ & 5.3782 & 0.4658 & 1.1588 \\
\hline $\mathrm{H}$ & 5.2034 & -3.0329 & 2.3760 \\
\hline $\mathrm{H}$ & 6.1595 & 0.2156 & 5.8387 \\
\hline $\mathrm{H}$ & 2.3641 & 6.2799 & -3.6126 \\
\hline $\mathrm{H}$ & -4.6449 & 4.7543 & -4.1895 \\
\hline $\mathrm{H}$ & -2.9512 & 4.6998 & -2.1629 \\
\hline $\mathrm{H}$ & -0.0987 & 8.1954 & -2.1529 \\
\hline $\mathrm{H}$ & 5.7043 & -1.3195 & 6.5890 \\
\hline $\mathrm{H}$ & 4.5042 & 0.0035 & 6.4914 \\
\hline $\mathrm{H}$ & 3.8457 & -0.9787 & 4.5410 \\
\hline $\mathrm{H}$ & 0.6986 & -1.8332 & 3.9191 \\
\hline $\mathrm{H}$ & -7.5250 & -3.0940 & 0.4750 \\
\hline $\mathrm{H}$ & -6.4092 & -2.5680 & -0.7637 \\
\hline $\mathrm{H}$ & 3.6508 & -0.5832 & 1.8648 \\
\hline $\mathrm{H}$ & -5.7630 & -3.3345 & 4.4137 \\
\hline $\mathrm{C}$ & -11.0112 & -4.9485 & 1.8483 \\
\hline $\mathrm{C}$ & -10.1651 & -4.1354 & 0.8908 \\
\hline 0 & -9.0652 & -3.6898 & 1.2333 \\
\hline $\mathrm{C}$ & -11.5346 & -4.0270 & 2.9567 \\
\hline $\mathrm{N}$ & -10.7176 & -3.8571 & -0.3104 \\
\hline C & -10.0630 & -2.9928 & -1.2751 \\
\hline $\mathrm{C}$ & -8.8628 & 7.0442 & 3.5857 \\
\hline $\mathrm{C}$ & -8.8681 & 5.8097 & 2.6916 \\
\hline $\mathrm{C}$ & -8.8695 & 6.1267 & 1.1980 \\
\hline $\mathrm{N}$ & -8.4927 & 5.0699 & 0.4188 \\
\hline 0 & -9.2211 & 7.2096 & 0.7395 \\
\hline $\mathrm{H}$ & -10.6978 & -3.5381 & 3.4609 \\
\hline $\mathrm{H}$ & -12.1058 & -4.5920 & 3.6982 \\
\hline $\mathrm{H}$ & -12.1851 & -3.2491 & 2.5452 \\
\hline $\mathrm{H}$ & -11.8401 & -5.4425 & 1.3288 \\
\hline $\mathrm{H}$ & -9.6270 & -2.1317 & -0.7636 \\
\hline $\mathrm{H}$ & -8.0215 & 5.1532 & 2.9037 \\
\hline $\mathrm{H}$ & -9.7661 & 5.2094 & 2.8889 \\
\hline $\mathrm{H}$ & -8.0077 & 4.2775 & 0.8378 \\
\hline $\mathrm{H}$ & -8.3738 & 5.2537 & -0.5662 \\
\hline $\mathrm{H}$ & -9.6862 & 7.7132 & 3.3233 \\
\hline $\mathrm{H}$ & -8.9567 & 6.7598 & 4.6383 \\
\hline $\mathrm{H}$ & -7.9338 & 7.6105 & 3.4677 \\
\hline $\mathrm{H}$ & -10.3720 & -5.7270 & 2.2745 \\
\hline $\mathrm{H}$ & -10.7986 & -2.6397 & -1.9992 \\
\hline $\mathrm{H}$ & -9.2615 & -3.5153 & -1.8107 \\
\hline $\mathrm{H}$ & -11.5992 & -4.2817 & -0.5499 \\
\hline C & 6.7741 & 0.3393 & -3.5593 \\
\hline C & 5.9010 & -0.7466 & -3.5569 \\
\hline $\mathrm{C}$ & 4.8916 & -0.8268 & -2.5970 \\
\hline C & 4.7459 & 0.1719 & -1.6275 \\
\hline $\mathrm{C}$ & 5.6607 & 1.2343 & -1.6104 \\
\hline C & 6.6549 & 1.3258 & -2.5770 \\
\hline $\mathrm{H}$ & 7.5629 & 0.4041 & -4.3032 \\
\hline $\mathrm{H}$ & 6.0196 & -1.5590 & -4.2624 \\
\hline $\mathrm{H}$ & 4.2402 & -1.6911 & -2.5871 \\
\hline $\mathrm{H}$ & 5.5656 & 1.9859 & -0.8387 \\
\hline $\mathrm{H}$ & 7.3537 & 2.1557 & -2.5569 \\
\hline & & & \\
\hline
\end{tabular}

\section{H $\quad 3.2002 \quad-0.8894-0.5561$ \\ $\begin{array}{llll}0 & 3.8556 & 0.7189 & 0.5433\end{array}$}

\section{TS1(BA)-S}

$\begin{array}{rrrr}\mathrm{C} & 11.0034 & 5.4250 & 0.4457 \\ \mathrm{C} & 9.5530 & 5.0549 & 0.0783 \\ \mathrm{C} & 9.0233 & 3.8198 & 0.7595 \\ \mathrm{C} & 8.2140 & 2.8247 & 0.2483 \\ \mathrm{~N} & 9.2558 & 3.5848 & 2.1030 \\ \mathrm{C} & 8.6002 & 2.4738 & 2.3886 \\ \mathrm{~N} & 7.9520 & 1.9676 & 1.3030 \\ \mathrm{H} & 11.0989 & 5.5220 & 1.5297 \\ \mathrm{H} & 9.4594 & 4.9298 & -1.0076 \\ \mathrm{H} & 8.9063 & 5.9046 & 0.3407\end{array}$

$2.6509-0.7356$

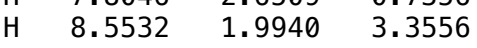

$\begin{array}{llll}\text { C } & -9.5418 & 2.0618 & -4.7699\end{array}$

C $\quad-8.6471 \quad 2.7337-3.7126$

$\begin{array}{llll}\text { C } & -7.5764 & 1.8135 & -3.1679\end{array}$

$\begin{array}{llll}\text { C } & -6.4499 & 1.4847 & -3.9381\end{array}$

C $-7.6703 \quad 1.2502-1.8905$

$\begin{array}{llll}C & -5.4696 & 0.6203 & -3.4618\end{array}$

C $-6.69310 .3873-1.3900$

$\begin{array}{llll}\text { C } & -5.5833 & 0.0603 & -2.1809\end{array}$

$\begin{array}{llll}0 & -4.5937 & -0.7770 & -1.7794\end{array}$

H $-10.0564 \quad 1.1939-4.3446$

$\begin{array}{llll}H & -9.2701 & 3.0950 & -2.8861\end{array}$

$\begin{array}{llll}\mathrm{H} & -8.1782 & 3.6206 & -4.1579\end{array}$

$\begin{array}{llll}\mathrm{H} & -6.3357 & 1.9176 & -4.9294\end{array}$

H $\quad-8.5065 \quad 1.5180-1.2496$

$\begin{array}{llll}\mathrm{H} & -4.5998 & 0.3721 & -4.0604\end{array}$

$\begin{array}{llll}H & -6.7592 & 0.0361 & -0.3674\end{array}$

$\mathrm{H}-4.6941-1.0880-0.8411$

$\begin{array}{llll}\text { C } & -0.1207 & -6.5214 & -5.3845\end{array}$

C $\quad-0.2997-6.2904-3.8806$

$\begin{array}{llll}\text { C } & -1.6284 & -5.6932 & -3.4478\end{array}$

$\begin{array}{llll}\text { C } & -2.0501 & -5.8178 & -2.1155\end{array}$

C $-2.4600-4.9753-4.3182$

$\begin{array}{llll}\text { C } & -3.2447 & -5.2589 & -1.6669\end{array}$

C $\quad-3.6631 \quad-4.4164 \quad-3.8886$

$\begin{array}{llll}\text { C } & -4.0675 & -4.5635 & -2.5598\end{array}$

$\begin{array}{llll}0 & -5.2794 & -4.0487 & -2.1983\end{array}$

H $-0.1226 \quad-5.5788-5.9416$

$\mathrm{H} \quad-0.1584 \quad-7.2404 \quad-3.3510$

H $\quad 0.5171 \quad-5.6424-3.5324$

$\begin{array}{llll}H & -1.4330 & -6.3655 & -1.4094\end{array}$

$\begin{array}{llll}H & -2.1727 & -4.8497 & -5.3569\end{array}$

$\begin{array}{llll}H & -3.5418 & -5.3687 & -0.6269\end{array}$

$\begin{array}{llll}\mathrm{H} & -4.3013 & -3.8647 & -4.5703\end{array}$

$\begin{array}{llll}\mathrm{H} & -5.3933 & -4.0992 & -1.2364\end{array}$

$\begin{array}{lll}5.0615 & -3.9865 & -5.3794\end{array}$

$\begin{array}{lll}5.1024 & -4.9836 & -4.2461\end{array}$

$5.8190 \quad-5.9811-4.2663$

$\begin{array}{lll}6.4520 & -3.7696 & -5.9808\end{array}$

$\begin{array}{lll}4.3824 & -4.3848 & -6.1446\end{array}$

$6.8691-4.7248-6.3065$

$\begin{array}{lll}7.1348 & -3.3480 & -5.2368\end{array}$

$\begin{array}{lll}6.4098 & -3.0872 & -6.8344\end{array}$

$\begin{array}{lll}4.2498 & -4.6971 & -3.2240\end{array}$

$4.2484-5.5034-2.0255$

$\begin{array}{lll}2.8706 & -5.5746 & -1.3702\end{array}$

$2.3146-4.2230-0.8965$

$0.8725 \quad-4.3854-0.4124$

$\begin{array}{lll}3.1914 & -3.5973 & 0.1898\end{array}$

$3.8739-3.7624 \quad-3.1714$

$\begin{array}{lll}4.9942 & -5.1277 & -1.3129\end{array}$

$\begin{array}{lll}2.9374 & -6.2474 & -0.5050\end{array}$

$2.1665-6.0360-2.0743$

$2.2960-3.5348-1.7547$

$\begin{array}{lll}0.8270 & -5.1149 & 0.4053\end{array}$

$\begin{array}{lll}0.4496 & -3.4563 & -0.0303\end{array}$

$0.2210-4.7398-1.2153$

$\begin{array}{lll}4.2545 & -3.6311 & -0.0661\end{array}$

$\begin{array}{lll}3.0649 & -4.1171 & 1.1437\end{array}$

$\begin{array}{lll}2.9408 & -2.5461 & 0.3426\end{array}$

$\begin{array}{lll}9.0985 & -5.2903 & -0.4835\end{array}$

$\begin{array}{lll}7.9973 & -4.4549 & -1.1608\end{array}$

$\begin{array}{lll}8.5110 & -3.1061 & -1.6096\end{array}$

$\begin{array}{lll}9.0972 & -2.9510 & -2.8717\end{array}$

\begin{tabular}{|c|c|c|c|}
\hline 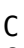 & 8.4593 & -1.9966 & -0.7565 \\
\hline & 9.6190 & -1.7195 & -3.2699 \\
\hline & 8.9776 & -0.7636 & -1.1491 \\
\hline & 9.5598 & -0.6227 & -2.4091 \\
\hline & 9.4950 & -4.7689 & 0.393 \\
\hline & 7.1687 & -4.3130 & -0.4561 \\
\hline & 7.5932 & -5.0054 & -2.0191 \\
\hline & 9.1422 & -3.8041 & -3.54 \\
\hline & 8.0031 & -2.0986 & 0.223 \\
\hline & 10.0670 & -1.6161 & -4.2545 \\
\hline & 8.9230 & 0.0859 & -0.4761 \\
\hline & 9.9569 & 0.3393 & -2.718 \\
\hline & -2.8940 & 0.8393 & 0.480 \\
\hline & -1.6821 & & \\
\hline & -1.6788 & 2.6280 & -0.50 \\
\hline & -0.5658 & 3.2907 & -0.769 \\
\hline & -2.8496 & 3.2836 & -0.741 \\
\hline & -3.9842 & 2.7572 & -0 \\
\hline & -4.0280 & 1.5841 & \\
\hline & 1.9784 & -0.4955 & -2.86 \\
\hline & 1.4509 & 0.5354 & -1.578 \\
\hline 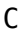 & -5.2558 & 3.2 & \\
\hline & 0.2927 & 0.0356 & -1.0490 \\
\hline & -0.2245 & -1.0992 & -1.710 \\
\hline & -1.5366 & -1.7010 & -1 \\
\hline & 0.5680 & -1.5055 & -2 \\
\hline & 0.3668 & -2.6104 & -3.73 \\
\hline & -0.4077 & 0.4896 & \\
\hline & 0.3530 & 2.9515 & 499 \\
\hline & -0.6063 & 4.2943 & -0. \\
\hline & -0.6393 & -0.4177 & 0.759 \\
\hline & 0.3106 & 1.0598 & \\
\hline & -5.9 & 31 & \\
\hline & -5.0447 & 4.5 & -0. \\
\hline & -5.7080 & 3.0266 & -1 \\
\hline & -3.0198 & -0.1152 & \\
\hline & -1.5 & -1 & \\
\hline & -1.6970 & -2.6154 & -1.9078 \\
\hline 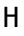 & -2.3643 & -1.0277 & -1.570 \\
\hline & 1.2694 & -3.2232 & -3 \\
\hline & 0.1 & -2 & \\
\hline & 1.1862 & -3.3061 & 7. \\
\hline[ & 0.4047 & -1.9952 & 7. \\
\hline - & 1.1467 & -0.8560 & \\
\hline ) & 1.4 & -1 & \\
\hline$)$ & 1.4483 & 0.1731 & 7. \\
\hline C & -8.8912 & 2.1787 & \\
\hline C & -7.8722 & 28 & \\
\hline C & -6.9 & 1.6 & 2. \\
\hline 0 & -7.1673 & 2.8044 & 1.6 \\
\hline 0 & -6.1038 & 0.8169 & 1. \\
\hline 1 & -9.4768 & 50 & \\
\hline & -8.3 & 3. & \\
\hline & -8.3724 & 0.2369 & 2. \\
\hline H & -7.1999 & 0.8326 & \\
\hline$C$ & -1.4704 & 2 & \\
\hline E & -1.1 & 1. & \\
\hline E & 0.1800 & 1.7084 & 3. \\
\hline$C$ & 0.5999 & 2.5655 & \\
\hline & & & \\
\hline C & 2.3 & 6 & \\
\hline & 1.9770 & 2.4903 & \\
\hline 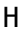 & -0.7068 & 97 & 6. \\
\hline 1 & -1.3 & 0.2 & \\
\hline & -1.9188 & 1.8445 & \\
\hline 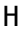 & 0.0023 & 3.2161 & 2. \\
\hline 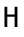 & 3.3952 & 2 & \\
\hline 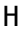 & 2.2 & & \\
\hline E & 4.8033 & 6.9 & \\
\hline E & 4.7955 & 5.4642 & 4 \\
\hline C & 5.5855 & & \\
\hline C & 5.6283 & 3.10 & \\
\hline C & 4.9969 & 4.2903 & \\
\hline t & 4. 3214 & & \\
\hline t & 3 & 5.1107 & 4.5100 \\
\hline & 5.1989 & 5.4446 & \\
\hline & 6.6184 & 4.8135 & \\
\hline & & 339 & \\
\hline & 5.9956 & 2.2946 & 0.4050 \\
\hline & 6.2731 & 3.1535 & \\
\hline & 3.9699 & 3.9117 & \\
\hline
\end{tabular}




\begin{tabular}{|c|c|c|c|}
\hline $\mathrm{H}$ & 5.6002 & 3.5866 & 1.3956 \\
\hline $\mathrm{H}$ & 4.9882 & 5.2406 & 1.4324 \\
\hline $\mathrm{H}$ & -0.4470 & -3.2650 & -3.4201 \\
\hline $\mathrm{H}$ & -9.5792 & 2.4689 & 2.9888 \\
\hline $\mathrm{H}$ & 11.7014 & 4.6469 & 0.1199 \\
\hline & 11.2942 & 6.3704 & -0.0238 \\
\hline $\mathrm{H}$ & -10.2977 & 2.7571 & -5.1499 \\
\hline r & -8.9452 & 1.7095 & -5.6177 \\
\hline $\mathrm{H}$ & -1.4783 & 2.5665 & 5.8327 \\
\hline $\mathrm{H}$ & -2.4447 & 1.0777 & 5.8478 \\
\hline $\mathrm{H}$ & 5.8279 & 7.2878 & 3.6346 \\
\hline $\mathrm{H}$ & 4.2686 & 7.5716 & 4.4244 \\
\hline $\mathrm{H}$ & -0.5752 & -2.1098 & 7.0371 \\
\hline 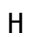 & 0.2295 & -1.7108 & 8.5625 \\
\hline H & 1.4174 & -3.5277 & 6.3730 \\
\hline $\mathrm{H}$ & 0.6277 & -4.1472 & 7.8449 \\
\hline $\mathrm{H}$ & 2.1381 & -3.2312 & 7.9553 \\
\hline $\mathrm{H}$ & 8.7144 & -6.2635 & -0.1606 \\
\hline $\mathrm{H}$ & 9.9307 & -5.4624 & -1.1735 \\
\hline $\mathrm{H}$ & 4.5852 & -6.4976 & -2.3245 \\
\hline $\mathrm{H}$ & 0.8357 & -7.0140 & -5.5810 \\
\hline $\mathrm{H}$ & -0.9202 & -7.1502 & -5.7898 \\
\hline $\mathrm{H}$ & 4.6368 & -3.0341 & -5.0431 \\
\hline $\mathrm{H}$ & 1.4041 & 0.3464 & 4.8449 \\
\hline $\mathrm{H}$ & -4.9616 & 1.2707 & 0.8762 \\
\hline C & 1.8486 & 6.9639 & -2.2708 \\
\hline C & 0.4962 & 6.7294 & -1.6474 \\
\hline 0 & 0.3282 & 5.8630 & -0.7867 \\
\hline $\mathrm{N}$ & -0.5185 & 7.5196 & -2.0966 \\
\hline C & -1.9025 & 7.1290 & -1.8746 \\
\hline C & -2.4081 & 6.4875 & -3.1674 \\
\hline 0 & -2.2241 & 7.0758 & -4.2367 \\
\hline $\mathrm{N}$ & -3.0032 & 5.2844 & -3.0429 \\
\hline C & -3.4499 & 4.5429 & -4.2122 \\
\hline C & -2.8987 & 3.1162 & -4.2507 \\
\hline C & -1.3699 & 2.9929 & -4.1951 \\
\hline $\mathrm{C}$ & -0.9818 & 1.5123 & -4.1522 \\
\hline C & -0.6718 & 3.7236 & -5.3477 \\
\hline C & 2.7891 & 2.6493 & -2.1780 \\
\hline C & 3.4199 & 3.7946 & -1.6515 \\
\hline $\mathrm{C}$ & 4.1253 & 4.6657 & -2.4751 \\
\hline $\mathrm{C}$ & 4.1616 & 4.4680 & -3.8571 \\
\hline C & 3.4690 & 3.3870 & -4.3982 \\
\hline C & 2.8035 & 2.4835 & -3.5690 \\
\hline $\mathrm{C}$ & 2.2939 & 1.6547 & -1.1594 \\
\hline 0 & 1.7865 & 2.3282 & -0.0563 \\
\hline $\mathrm{H}$ & 1.9286 & 7.9394 & -2.7582 \\
\hline $\mathrm{H}$ & 2.6126 & 6.8713 & -1.4998 \\
\hline $\mathrm{H}$ & -1.9366 & 6.4619 & -1.0132 \\
\hline $\mathrm{H}$ & -2.5103 & 8.0140 & -1.6615 \\
\hline $\mathrm{H}$ & -3.3318 & 2.5449 & -3.4226 \\
\hline $\mathrm{H}$ & -3.2694 & 2.6427 & -5.1710 \\
\hline $\mathrm{H}$ & -1.0263 & 3.4486 & -3.2602 \\
\hline $\mathrm{H}$ & 0.4145 & 3.6064 & -5.2720 \\
\hline $\mathrm{H}$ & -0.9883 & 3.3205 & -6.3184 \\
\hline $\mathrm{H}$ & -0.8924 & 4.7954 & -5.3389 \\
\hline $\mathrm{H}$ & -1.3082 & 0.9932 & -5.0623 \\
\hline $\mathrm{H}$ & -1.4438 & 1.0049 & -3.2993 \\
\hline $\mathrm{H}$ & 0.1002 & 1.3928 & -4.0708 \\
\hline $\mathrm{H}$ & -3.1405 & 5.1213 & -5.0838 \\
\hline $\mathrm{H}$ & 3.3690 & 3.9816 & -0.5867 \\
\hline $\mathrm{H}$ & 4.6434 & 5.5105 & -2.0316 \\
\hline $\mathrm{H}$ & 4.7110 & 5.1499 & -4.4987 \\
\hline $\mathrm{H}$ & 3.4618 & 3.2227 & -5.4716 \\
\hline $\mathrm{H}$ & 2.3172 & 1.6368 & -4.0311 \\
\hline C & -5.4191 & -3.6499 & 3.1216 \\
\hline $\mathrm{C}$ & -4.0654 & -3.0508 & 3.5379 \\
\hline 0 & -3.9857 & -1.8911 & 3.9356 \\
\hline C & -6.2774 & -2.6055 & 2.3846 \\
\hline $\mathrm{C}$ & -5.8260 & -2.3271 & 0.9649 \\
\hline $\mathrm{N}$ & -6.6746 & -2.7136 & -0.0203 \\
\hline 0 & -4.7462 & -1.7767 & 0.7001 \\
\hline $\mathrm{N}$ & -2.9865 & -3.8790 & 3.5290 \\
\hline C & -1.6865 & -3.3947 & 3.9764 \\
\hline C & -0.9530 & -2.6194 & 2.8756 \\
\hline 0 & -1.2658 & -2.7402 & 1.6837 \\
\hline $\mathrm{C}$ & -0.9089 & -4.7015 & 4.2394 \\
\hline 0 & -1.4259 & -5.6165 & 3.1173 \\
\hline C & -2.9105 & -5.2328 & 2.9671 \\
\hline $\mathrm{N}$ & 0.1130 & -1.9253 & 3.3104 \\
\hline & & & \\
\hline
\end{tabular}

\begin{tabular}{|c|c|c|c|}
\hline & 958 & -2.2771 & \\
\hline & & 885 & \\
\hline & 940 & 5 & \\
\hline & & -1 & \\
\hline & 74 & -1.3515 & \\
\hline & & -1 & \\
\hline & & -1 & \\
\hline & & & \\
\hline & & -0 . & \\
\hline & & -0 . & \\
\hline & -7.3 & -2 & \\
\hline & & -1.6488 & \\
\hline & & -4 & \\
\hline & 9 & -5 . & \\
\hline & & -4 & \\
\hline & -1 & -6 & \\
\hline & -0.8 & -5 & \\
\hline & -3 & -5 & \\
\hline & -3.2 & -5 & \\
\hline & -1. & -2 & \\
\hline & & -0 & \\
\hline & & -2 & \\
\hline & & -1 & \\
\hline & & -2 & \\
\hline & 5. & & \\
\hline & & -3 & \\
\hline & & & \\
\hline & & & \\
\hline & -4 & & \\
\hline & -2 & & \\
\hline & -0. & & -2 \\
\hline & & -1 & \\
\hline & & & \\
\hline & & -0 & \\
\hline & & -1 & \\
\hline & -7 . & -3 & \\
\hline & -6. & -2 & \\
\hline & 3. & -0.6735 & \\
\hline & -5 & -3 & \\
\hline & -10 & -5 & \\
\hline & -10 & -4 & \\
\hline & 71 & -4 & \\
\hline$C$ & -11. & -4.7361 & \\
\hline & -10.4793 & -4.4586 & -1 \\
\hline & -9 . & -3 & -1 \\
\hline & -9. & & \\
\hline & -9 . & & \\
\hline$C$ & -9 . & & \\
\hline & -8 & & \\
\hline & -9.6789 & & \\
\hline & -10 & -4 & \\
\hline & -12 & -5 & \\
\hline & -12 & -3 & \\
\hline & -11 & -6 & \\
\hline & -9.7 & -2 & -1 \\
\hline & -8.5 & & \\
\hline & -10.2 & & \\
\hline & -8. & & \\
\hline & -8 & & \\
\hline & -10 & & \\
\hline & & & \\
\hline & -8.6 & & \\
\hline & -10. & -6 . & \\
\hline & -10 & -3 & \\
\hline & -8 & -3 & -2 \\
\hline & -11 & -4 & \\
\hline & & -0. & \\
\hline & & -1 & \\
\hline & & -1 & \\
\hline & & & \\
\hline & & & \\
\hline & & & \\
\hline & & -1 & -4 \\
\hline & & -2.6585 & \\
\hline & 4 & -1.6511 & \\
\hline & & & \\
\hline & & & \\
\hline & & & \\
\hline & & & \\
\hline & & & \\
\hline & & & \\
\hline
\end{tabular}

Int1(BA)-R

\begin{tabular}{|c|c|c|c|}
\hline & 11.2763 & 4.8525 & -0.7970 \\
\hline & 9.9055 & 5.0313 & -0.1245 \\
\hline & 996 & 778 & 29 \\
\hline & 8.3037 & & \\
\hline & 10.0446 & 110 & של \\
\hline & 0 & 346 & D196 \\
\hline & & & \\
\hline & 12.0049 & & -0.0656 \\
\hline & & & \\
\hline & 9. & & \\
\hline & & & \\
\hline & 9.5 & & \\
\hline & & & 81 \\
\hline & -9 . & & \\
\hline & -8 & & \\
\hline & -7.7 & & \\
\hline & -6.7 & & \\
\hline & -7 . & & \\
\hline & -5 & & \\
\hline & -6. & & \\
\hline & -5 & & \\
\hline & -5. & 0 & \\
\hline & -10.3 & & \\
\hline & -9.2 & & \\
\hline & -8. & & \\
\hline & -6 & & \\
\hline & -8.4 & & -0. \\
\hline & -5. & & \\
\hline & & & \\
\hline & -4 & -1 . & \\
\hline & -0. & -6 . & -5 \\
\hline & -0 . & & \\
\hline & -2 & & \\
\hline & -2 & -5 . & \\
\hline & -3. & & \\
\hline & -3 & & \\
\hline & -4 & & \\
\hline & -4.7 & -5 . & -2 \\
\hline J & -5 & -5 & \\
\hline & & & \\
\hline & -0 . & & \\
\hline & -0. & -5 . & -3 \\
\hline & -1 & & \\
\hline & & & \\
\hline & -3. & & \\
\hline & -5 & -6 . & -3 \\
\hline & -5 . & & \\
\hline & & & \\
\hline & & & \\
\hline & 5.2. & -3 & -3 \\
\hline & & & \\
\hline & & & \\
\hline & & & \\
\hline & & & \\
\hline & & & \\
\hline & & & \\
\hline & 3.7 & & -2 \\
\hline & & -5 & \\
\hline & & & \\
\hline & & & \\
\hline & & & \\
\hline & & & \\
\hline & & & \\
\hline & & & \\
\hline & & & \\
\hline & & & \\
\hline & & & \\
\hline & -0. & & \\
\hline & & & \\
\hline t & & & \\
\hline & & & \\
\hline & & & \\
\hline & & & \\
\hline & & & -0 \\
\hline & & & \\
\hline & 10 & & -1.2407 \\
\hline & & & \\
\hline
\end{tabular}




\begin{tabular}{|c|c|c|c|c|c|c|c|}
\hline C & 8.8849 & -1.0882 & -0.8335 & H & 5.4798 & 4.7405 & 0.9172 \\
\hline C & 9.7198 & -2.2215 & 1.1249 & $\mathrm{H}$ & -1.2136 & -3.2920 & -3.6810 \\
\hline C & 9.6304 & -1.0670 & 0.3452 & $\mathrm{H}$ & -9.1666 & 3.1045 & 3.5652 \\
\hline $\mathrm{H}$ & 9.4154 & -5.9945 & -0.5628 & $\mathrm{H}$ & 11.2194 & 4.1127 & -1.6026 \\
\hline $\mathrm{H}$ & 7.0873 & -5.1327 & -0.1076 & H & 11.6418 & 5.7952 & -1.2195 \\
\hline $\mathrm{H}$ & 6.9953 & -4.5013 & -1.7514 & $\mathrm{H}$ & -10.5120 & 3.3302 & -4.5004 \\
\hline $\mathrm{H}$ & 7.6614 & -2.2694 & -2.1525 & $\mathrm{H}$ & -9.2912 & 2.1941 & -5.1019 \\
\hline $\mathrm{H}$ & 9.1342 & -4.2793 & 1.3415 & $\mathrm{H}$ & -0.9150 & 2.7740 & 5.5826 \\
\hline $\mathrm{H}$ & 8.7804 & -0.1864 & -1.4268 & $\mathrm{H}$ & -1.8288 & 1.2786 & 5.8719 \\
\hline $\mathrm{H}$ & 10.2697 & -2.2052 & 2.0608 & H & 6.4319 & 7.0126 & 2.5426 \\
\hline $\mathrm{H}$ & 10.1167 & -0.1520 & 0.6635 & $\mathrm{H}$ & 5.1373 & 7.5316 & 3.6336 \\
\hline C & -2.9840 & 0.8453 & 0.5692 & H & -0.0975 & -2.0759 & 6.5388 \\
\hline C & -1.7618 & 1.1610 & 0.0308 & $\mathrm{H}$ & 0.6409 & -1.5295 & 8.0545 \\
\hline C & -1.6481 & 2.4758 & -0.5373 & $\mathrm{H}$ & 1.8830 & -3.5833 & 6.1239 \\
\hline $\mathrm{N}$ & -0.4805 & 3.0149 & -0.8423 & $\mathrm{H}$ & 1.0543 & -4.0137 & 7.6384 \\
\hline $\mathrm{N}$ & -2.7686 & 3.2110 & -0.7877 & $\mathrm{H}$ & 2.5714 & -3.0984 & 7.6767 \\
\hline C & -3.9296 & 2.8032 & -0.3069 & $\mathrm{H}$ & 8.2427 & -6.6824 & -1.7002 \\
\hline $\mathrm{N}$ & -4.0516 & 1.6791 & 0.4384 & $\mathrm{H}$ & 9.3173 & -5.3666 & -2.2111 \\
\hline $\mathrm{S}$ & 1.4749 & -0.7637 & -3.1586 & $\mathrm{H}$ & 4.2999 & -6.6684 & -2.5918 \\
\hline C & 1.1568 & 0.1786 & -1.7426 & $\mathrm{H}$ & 0.0542 & -6.8397 & -5.8228 \\
\hline C & -5.1546 & 3.5973 & -0.6056 & $\mathrm{H}$ & -1.6700 & -7.2015 & -5.9151 \\
\hline $\mathrm{N}$ & -0.0047 & -0.2340 & -1.1670 & $\mathrm{H}$ & 3.3003 & -4.3253 & -6.2190 \\
\hline C & -0.6771 & -1.2590 & -1.8730 & $\mathrm{H}$ & 1.8454 & 0.4438 & 4.2728 \\
\hline C & -2.0217 & -1.7304 & -1.4420 & $\mathrm{H}$ & -4.9885 & 1.4482 & 1.0012 \\
\hline C & -0.0038 & -1.6659 & -2.9793 & C & 2.0198 & 6.8601 & -2.7571 \\
\hline C & -0.3819 & -2.6728 & -4.0195 & C & 0.6758 & 6.3286 & -2.3270 \\
\hline C & -0.5870 & 0.2247 & 0.1506 & 0 & 0.4306 & 5.1208 & -2.3405 \\
\hline H & 0.3939 & 2.6217 & -0.4817 & $\mathrm{~N}$ & -0.2322 & 7.2356 & -1.8717 \\
\hline $\mathrm{H}$ & -0.4175 & 3.9163 & -1.3177 & C & -1.6063 & 6.8042 & -1.6771 \\
\hline $\mathrm{H}$ & -0.8914 & -0.6862 & 0.6651 & C & -2.2292 & 6.4038 & -3.0249 \\
\hline $\mathrm{H}$ & 0.2185 & 0.6897 & 0.7051 & 0 & -2.0075 & 7.0789 & -4.0284 \\
\hline $\mathrm{H}$ & -5.8516 & 3.5511 & 0.2366 & $\mathrm{~N}$ & -3.0170 & 5.3058 & -2.9824 \\
\hline $\mathrm{H}$ & -4.8801 & 4.6278 & -0.8324 & C & -3.5479 & 4.7111 & -4.2019 \\
\hline $\mathrm{H}$ & -5.6511 & 3.1664 & -1.4811 & C & -3.1007 & 3.2523 & -4.3617 \\
\hline $\mathrm{H}$ & -3.1751 & -0.0746 & 1.1137 & C & -1.5830 & 3.0312 & -4.2722 \\
\hline $\mathrm{H}$ & -2.0243 & -2.0883 & -0.4104 & C & -1.2621 & 1.5348 & -4.2742 \\
\hline $\mathrm{H}$ & -2.3451 & -2.5452 & -2.0862 & C & -0.8012 & 3.7729 & -5.3613 \\
\hline $\mathrm{H}$ & -2.7615 & -0.9362 & -1.5471 & C & 2.7337 & 2.1848 & -2.1697 \\
\hline $\mathrm{H}$ & 0.4595 & -3.3373 & -4.2431 & C & 3.2874 & 3.3081 & -1.5291 \\
\hline $\mathrm{H}$ & -0.6807 & -2.1820 & -4.9527 & C & 4.0417 & 4.2339 & -2.2420 \\
\hline C & 1.6324 & -3.2340 & 7.1300 & C & 4.2293 & 4.0924 & -3.6161 \\
\hline C & 0.8584 & -1.9128 & 7.0541 & C & 3.6416 & 3.0109 & -4.2687 \\
\hline C & 1.6544 & -0.8473 & 6.2889 & C & 2.9105 & 2.0635 & -3.5534 \\
\hline 0 & 1.8208 & -1.0865 & 5.0221 & C & 2.1403 & 1.1410 & -1.2530 \\
\hline 0 & 2.0986 & 0.1385 & 6.8880 & 0 & 1.7519 & 1.6905 & -0.0500 \\
\hline C & -8.4073 & 2.7527 & 4.2654 & H & 1.9973 & 7.9246 & -3.0049 \\
\hline C & -7.5905 & 1.6097 & 3.6568 & H & 2.7347 & 6.7010 & -1.9460 \\
\hline C & -6.8264 & 1.9733 & 2.3769 & $\mathrm{H}$ & -1.6371 & 5.9854 & -0.9552 \\
\hline 0 & -6.9697 & 3.1165 & 1.8856 & $\mathrm{H}$ & -2.1726 & 7.6414 & -1.2609 \\
\hline 0 & -6.0709 & 1.0524 & 1.9062 & H & -3.5939 & 2.6446 & -3.5923 \\
\hline $\mathrm{H}$ & -8.9090 & 2.4237 & 5.1821 & $\mathrm{H}$ & -3.4767 & 2.8846 & -5.3273 \\
\hline $\mathrm{H}$ & -7.7665 & 3.6016 & 4.5130 & $\mathrm{H}$ & -1.2423 & 3.4350 & -3.3166 \\
\hline $\mathrm{H}$ & -8.2327 & 0.7510 & 3.4200 & $\mathrm{H}$ & 0.2728 & 3.6021 & -5.2346 \\
\hline $\mathrm{H}$ & -6.8494 & 1.2295 & 4.3695 & $\mathrm{H}$ & -1.0889 & 3.4261 & -6.3627 \\
\hline C & -0.9075 & 1.6882 & 5.4437 & $\mathrm{H}$ & -0.9683 & 4.8521 & -5.3101 \\
\hline C & -0.8026 & 1.3434 & 3.9491 & $\mathrm{H}$ & -1.5783 & 1.0594 & -5.2118 \\
\hline C & 0.4758 & 1.8464 & 3.3624 & H & -1.7692 & 1.0169 & -3.4526 \\
\hline C & 0.7772 & 2.9423 & 2.5805 & $\mathrm{H}$ & -0.1869 & 1.3748 & -4.1633 \\
\hline $\mathrm{N}$ & 1.6993 & 1.2906 & 3.6826 & $\mathrm{H}$ & -3.2037 & 5.3343 & -5.0289 \\
\hline C & 2.6602 & 2.0495 & 3.0956 & $\mathrm{H}$ & 3.1513 & 3.4364 & -0.4631 \\
\hline $\mathrm{N}$ & 2.1431 & 3.0629 & 2.4142 & H & 4.4964 & 5.0634 & -1.7084 \\
\hline $\mathrm{H}$ & -0.0563 & 1.2899 & 6.0022 & $\mathrm{H}$ & 4.8273 & 4.8103 & -4.1697 \\
\hline $\mathrm{H}$ & -0.8866 & 0.2591 & 3.8139 & $\mathrm{H}$ & 3.7670 & 2.8842 & -5.3398 \\
\hline $\mathrm{H}$ & -1.6411 & 1.7882 & 3.4015 & $\mathrm{H}$ & 2.4975 & 1.2277 & -4.1011 \\
\hline $\mathrm{H}$ & 0.0878 & 3.6457 & 2.1330 & C & -5.3237 & -3.2667 & 3.4125 \\
\hline $\mathrm{H}$ & 3.7092 & 1.8081 & 3.1675 & C & -3.8912 & -2.7266 & 3.5982 \\
\hline $\mathrm{H}$ & 2.5599 & 1.5584 & 0.5608 & 0 & -3.7040 & -1.5457 & 3.8836 \\
\hline C & 5.4684 & 6.7192 & 2.9774 & C & -6.1730 & -2.2933 & 2.5730 \\
\hline C & 5.5866 & 5.4008 & 3.7458 & C & -5.7523 & -2.2335 & 1.1203 \\
\hline C & 6.1381 & 4.2130 & 2.9346 & $\mathrm{~N}$ & -6.5784 & -2.8155 & 0.2059 \\
\hline C & 6.1985 & 2.9687 & 3.8336 & 0 & -4.6973 & -1.6892 & 0.7588 \\
\hline C & 5.3046 & 3.9616 & 1.6685 & $\mathrm{~N}$ & -2.8626 & -3.6131 & 3.5050 \\
\hline $\mathrm{H}$ & 4.7456 & 6.6404 & 2.1596 & C & -1.4970 & -3.2030 & 3.8240 \\
\hline $\mathrm{H}$ & 4.5951 & 5.1208 & 4.1270 & C & -0.7708 & -2.5789 & 2.6233 \\
\hline $\mathrm{H}$ & 6.2247 & 5.5512 & 4.6270 & 0 & -1.1941 & -2.7239 & 1.4648 \\
\hline $\mathrm{H}$ & 7.1654 & 4.4487 & 2.6214 & C & -0.8119 & -4.5413 & 4.1693 \\
\hline $\mathrm{H}$ & 5.2251 & 2.7752 & 4.2970 & C & -1.4784 & -5.5103 & 3.1802 \\
\hline $\mathrm{H}$ & 6.4876 & 2.0734 & 3.2775 & C & -2.9375 & -5.0247 & 3.1036 \\
\hline $\mathrm{H}$ & 6.9201 & 3.1094 & 4.6464 & $\mathrm{~N}$ & 0.3794 & -1.9688 & 2.9421 \\
\hline $\mathrm{H}$ & 4.2327 & 3.9358 & 1.8884 & C & 1.3961 & -1.6423 & 1.9670 \\
\hline $\mathrm{H}$ & 5.5715 & 3.0052 & 1.2132 & C & 2.7164 & -2.3479 & 2.3111 \\
\hline
\end{tabular}

\begin{tabular}{|c|c|c|c|}
\hline & 2.7532 & -3.5421 & 2.6103 \\
\hline & 903 & -1.5321 & 2180 \\
\hline & 651 & 415 & 工 \\
\hline & 72 & 46 & \\
\hline & 6.8923 & -1.7617 & 1038 \\
\hline & 207 & -1.3368 & 1.4095 \\
\hline & 6.2126 & 809 & 51 \\
\hline & 520 & -0.9461 & 588 \\
\hline & .3328 & -0.4871 & 5.979 \\
\hline & & & \\
\hline & 72 & & \\
\hline & 38 & & \\
\hline & -1.0422 & -4 & 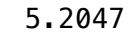 \\
\hline & & & \\
\hline & -1.2 & & \\
\hline & -1.6 & & \\
\hline & -3 & -5 & \\
\hline & -3 & & \\
\hline & -1.5 & & \\
\hline & 478 & -0. & \\
\hline & 1.0536 & -2 & \\
\hline & & & \\
\hline & 5.808 & -1 . & \\
\hline & 5.3782 & 58 & \\
\hline & & & \\
\hline & 6 & & \\
\hline & & & -3 . \\
\hline & -4.6 & & -4 \\
\hline & -2 & & \\
\hline & -0. & & -2 \\
\hline & 5.7 & -1 & \\
\hline & & & \\
\hline & & & \\
\hline & 0.6 & -1 & \\
\hline & -7.5 & -3 & 0. \\
\hline & -6. & -2 & \\
\hline & & & \\
\hline & -5.7 & -3 & \\
\hline$C$ & $-11 . e$ & -4 & \\
\hline C & -10 . & -4 & \\
\hline ) & -9. & & \\
\hline & -11.5 & -4 & 2. \\
\hline & -10. & -3 & -0. \\
\hline 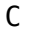 & -10. & & \\
\hline E & -8. & & \\
\hline & -8.8 & & \\
\hline$C$ & $-8 . \varepsilon$ & & \\
\hline & & & \\
\hline & -9. & & \\
\hline & -10.69 & -3 & 3. \\
\hline | & -12.1 & -4 & \\
\hline 1 & -12 & & \\
\hline & -11 & -5 & \\
\hline & -9.62 & -2 . & -0.7 \\
\hline H & & & \\
\hline & & & \\
\hline & -8 & & \\
\hline 1 & -8 & & -0. \\
\hline & & & \\
\hline & -8 & & \\
\hline & -7. & & \\
\hline 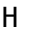 & -10.3 & -5 & \\
\hline & -10 & & \\
\hline & -9.2 & -3 & \\
\hline & -11. & -4 & -0 . \\
\hline C & 6. & & \\
\hline$C$ & & & \\
\hline & 4.8 & & \\
\hline$r$ & & & -1 \\
\hline C & 5.6 & & -1 . \\
\hline C & & & \\
\hline & & & \\
\hline & & & \\
\hline H & 4.2402 & -1 & -2.5871 \\
\hline $\mathrm{H}$ & & & -0 . \\
\hline & & & \\
\hline & & & \\
\hline & & -0. & -0. \\
\hline & & & 0.543 \\
\hline
\end{tabular}




\section{Int1(BA)-S}

\begin{tabular}{|c|c|c|c|}
\hline & 11.0518 & 5.4470 & דלעם הט \\
\hline & 9.5969 & 5.0903 & -0.202 \\
\hline & 301 & 9000 & 28 \\
\hline & 1793 & 182 & 059 \\
\hline & 9.2585 & 7084 & + \\
\hline & 8.5590 & 6362 & 2.20 \\
\hline & 865 & 140 & \\
\hline & 11.1408 & 917 & 1. \\
\hline & & & -1 \\
\hline & 8.9678 & 5. & \\
\hline & 7.760 & 2 . & -0 \\
\hline & 8.4962 & & \\
\hline & -9.5264 & 1. & -4 \\
\hline & -8.6423 & 2.62 & -3 \\
\hline & -7. & $1-$ & -3 \\
\hline & -6 & $1.3 e$ & -3 \\
\hline & -7.6 & 1.2 & -1 . \\
\hline & -5 . & & -3 \\
\hline & -6 & 0.3 & -1 \\
\hline & -5 . & -0.0 & -2 . \\
\hline & -4 & -0 . & -1 \\
\hline & -10.04 & 1. & -4 \\
\hline & -9.270 & 3. & -2 \\
\hline & -8. & & -4 \\
\hline & -6. & 1. & -4 \\
\hline & -8.4631 & 1.5576 & -1 \\
\hline & -4. & & -4 \\
\hline & -6.7286 & 0.0 & -0. \\
\hline & -4 & -1.08 & -0 . \\
\hline & -0 & & -5 \\
\hline & -0. & -6 & -3 \\
\hline & -1 & -5.98 & -3 \\
\hline & -2 & & -1 \\
\hline & -2 . & & -4 \\
\hline & -3. & -5.2 & -1 . \\
\hline & -3 & -4 & -3 \\
\hline & -4 & -4 & -2 \\
\hline & -5. & -4.3 & -2 \\
\hline & -0. & -5 & -5 \\
\hline & -0. & -7 & -3 \\
\hline & 0.35 & $-6 . e$ & -3 \\
\hline & -1.4 & -6 & -1 \\
\hline & -2 & -5 & -5 \\
\hline & -3 & -5.2 & -0 . \\
\hline & -4.6 & -4 & -4 \\
\hline & -5 . & -4 & -1 \\
\hline & 5. & -4.19 & -5 \\
\hline & 4. & -5.2 & -4 \\
\hline & 4. & -6 & -4 \\
\hline & 6. & -4.2 & -5 \\
\hline & 4. & -4. & -6 \\
\hline & 6 & -5 & -5 \\
\hline & 7. & -3 & -4 \\
\hline & 6. & -3 & -6 \\
\hline & & -4 & -2 \\
\hline & 4. & -5 & -1 \\
\hline & 2. & -5 & -1 \\
\hline & & & -0 \\
\hline & 0. & -4 & -0 \\
\hline & 3. & -3 & \\
\hline & & & \\
\hline & 4. & -5 & -1 \\
\hline & & -6 & -0 \\
\hline & & & -1 \\
\hline & & -3 & -1 \\
\hline & & & \\
\hline & 0. & & \\
\hline & 0. & -4 & -0 \\
\hline & & & \\
\hline & 3. & -4 & \\
\hline & & -2.5320 & \\
\hline & & -5.2 & -0 \\
\hline & & -4.32 & -0 \\
\hline & 69 & -3.0266 & -1 \\
\hline & & -2.95 & -2 \\
\hline & & -1.87 & \\
\hline & & & \\
\hline
\end{tabular}

$\begin{array}{llll}\text { C } & 9.1633 & -0.6709 & -0.9694\end{array}$

$\begin{array}{lll}9.8128 & -0.6098 & -2.2022\end{array}$

$\begin{array}{lll}9.6006 & -4.8415 & 0.5960\end{array}$

$\begin{array}{lll}7.2940 & -4.1165 & -0.1064\end{array}$

$\begin{array}{lll}7.5547 & -4.8179 & -1.6982\end{array}$

$\begin{array}{lll}9.4094 & -3.8445 & -3.1782\end{array}$

$8.0861 \quad-1.9105 \quad 0.4156$

$10.4107-1.7167-3.9542$

$\begin{array}{lll}9.0808 & 0.2169 & -0.3512\end{array}$

$\begin{array}{lll}10.2393 & 0.3281 & -2.5446\end{array}$

$\begin{array}{lll}-2.8537 & 0.8355 & 0.4728\end{array}$

$\begin{array}{lll}-1.6457 & 1.2221 & -0.0610\end{array}$

$\begin{array}{lll}-1.6246 & 2.5284 & -0.6587\end{array}$

$\begin{array}{lll}-0.5045 & 3.1382 & -1.0053\end{array}$

$\begin{array}{llll}\mathrm{N} & -2.7855 & 3.2001 & -0.8941\end{array}$

$\begin{array}{llll}\text { C } & -3.9206 & 2.7266 & -0.4166\end{array}$

$\begin{array}{llll}N & -3.9751 & 1.5952 & 0.3288\end{array}$

$\begin{array}{lll}2.0003 & -0.8893 & -2.7571\end{array}$

$\begin{array}{lll}1.4941 & 0.2365 & -1.5644\end{array}$

$\begin{array}{llll}-5.1807 & 3.4543 & -0.7295\end{array}$

$\begin{array}{lll}0.2917 & -0.1325 & -1.0666\end{array}$

$\begin{array}{lll}-0.2732 & -1.2810 & -1.6693\end{array}$

$\begin{array}{lll}-1.6172 & -1.7962 & -1.2776\end{array}$

$\begin{array}{lll}0.5352 & -1.8079 & -2.6287\end{array}$

$0.3120-2.9856-3.5239$

$\begin{array}{lll}-0.3869 & 0.4163 & 0.1660\end{array}$

$\begin{array}{llll}\mathrm{H} & 0.4144 & 2.7999 & -0.6823\end{array}$

$\begin{array}{llll}\mathrm{H} & -0.5258 & 4.1308 & -1.2331\end{array}$

$\begin{array}{llll}\text { H } & -0.6250 & -0.4577 & 0.7727\end{array}$

$\begin{array}{llll}\mathrm{H} & 0.3569 & 1.0015 & 0.6917\end{array}$

$\begin{array}{llll}H & -5.8777 & 3.4028 & 0.1134\end{array}$

H $\quad-4.9483 \quad 4.4881-0.9860$

$\begin{array}{llll}H & -5.6608 & 2.9774 & -1.5905\end{array}$

$\begin{array}{llll}H & -2.9847 & -0.0819 & 1.0395\end{array}$

$\begin{array}{llll}\mathrm{H} & -1.6884 & -1.9949 & -0.2058\end{array}$

$\begin{array}{llll}\mathrm{H} & -1.8040 & -2.7394 & -1.7907\end{array}$

H $\quad-2.4118-1.1075-1.5709$

$\begin{array}{lll}1.2070 & -3.6126 & -3.5817\end{array}$

$\begin{array}{lll}0.0598 & -2.6628 & -4.5399\end{array}$

$\begin{array}{lll}1.2298 & -2.9162 & 7.5641\end{array}$

$\begin{array}{lll}0.4464 & -1.6036 & 7.6193\end{array}$

$\begin{array}{lll}1.1820 & -0.4927 & 6.8652\end{array}$

$\begin{array}{lll}1.4403 & -0.7677 & 5.6203\end{array}$

$\begin{array}{lll}1.4845 & 0.5614 & 7.4345\end{array}$

$\begin{array}{lll}-8.8413 & 2.4354 & 3.7203\end{array}$

$\begin{array}{lll}-7.8264 & 1.3787 & 3.2713\end{array}$

$\begin{array}{llll}C & -6.9208 & 1.8104 & 2.1094\end{array}$

$\begin{array}{llll}0 & -7.0718 & 2.9562 & 1.6238\end{array}$

$\begin{array}{llll}0 & -6.0563 & 0.9468 & 1.7289\end{array}$

$\begin{array}{llll}H & -9.4388 & 2.0635 & 4.5600\end{array}$

$\begin{array}{llll}H & -8.3379 & 3.3507 & 4.0372\end{array}$

$\begin{array}{llll}\mathrm{H} & -8.3312 & 0.4528 & 2.9665\end{array}$

$\begin{array}{llll}\mathrm{H} & -7.1654 & 1.0894 & 4.0971\end{array}$

$\begin{array}{lll}\text { C }-1.4160 & 1.8112 & 5.5211\end{array}$

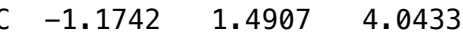

$\begin{array}{lll}0.1845 & 1.8687 & 3.5465\end{array}$

$\begin{array}{lll}0.5855 & 2.6349 & 2.4757\end{array}$

$\begin{array}{llll}\mathrm{N} & 1.3522 & 1.3616 & 4.0822\end{array}$

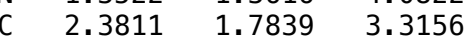

$\begin{array}{llll}N & 1.9623 & 2.5608 & 2.3229\end{array}$

$\begin{array}{llll}\mathrm{H} & -0.6453 & 1.3742 & 6.1612\end{array}$

$\begin{array}{llll}H & -1.3278 & 0.4167 & 3.8807\end{array}$

$\begin{array}{llll}\text { H } & -1.9173 & 2.0017 & 3.4206\end{array}$

$\mathrm{H}-0.0231 \quad 3.2265 \quad 1.8066$

$\begin{array}{llll}\mathrm{H} & 3.4025 & 1.4864 & 3.4915\end{array}$

$\begin{array}{llll}\mathrm{H} & 2.2287 & 2.1547 & 0.7072\end{array}$

$\begin{array}{llll}\text { C } & 4.8704 & 7.1057 & 3.4011\end{array}$

$\begin{array}{lll}4.8550 & 5.6833 & 3.9699\end{array}$

$\begin{array}{lll}5.5737 & 4.6129 & 3.1247\end{array}$

$\begin{array}{lll}5.6091 & 3.2925 & 3.9114\end{array}$

$\begin{array}{lll}4.9206 & 4.4165 & 1.7481\end{array}$

$\begin{array}{lll}4.3354 & 7.1653 & 2.4478\end{array}$

$3.8144 \quad 5.3623 \quad 4.1163$

$5.3112 \quad 5.6968 \quad 4.9690$

$\begin{array}{lll}6.6111 & 4.9376 & 2.9596\end{array}$

$\begin{array}{lll}4.6198 & 3.0651 & 4.3201\end{array}$

$\begin{array}{lll}5.9121 & 2.4464 & 3.2887\end{array}$

$\begin{array}{lll}6.3021 & 3.3555 & 4.7576\end{array}$

$\begin{array}{lll}3.8852 & 4.0725 & 1.8475\end{array}$

$\begin{array}{lll}5.4762 & 3.6684 & 1.1730\end{array}$
H 11.7362

11.3661

H -10.2775

H -8.9223

H -1.4009

H -2.3902

H $\quad 5.8968$

$\begin{array}{rr}\mathrm{H} & 4.3935 \\ \mathrm{H} & -0.5367\end{array}$

H -0.5367

$\begin{array}{lll}\mathrm{H} & 0.2781 \\ \mathrm{H} & 1.4576\end{array}$

H $\quad 0.6750-3.7411$

H $2.1838-2.8184$

H $\quad 8.6372-6.2292$

H $\quad 9.8742 \quad-5.5302$

H $4.5378-6.5567$

H $\quad 0.8443 \quad-7.1608$

$\mathrm{H}-0.8803$

$\begin{array}{rr}\mathrm{H} & 4.7550 \\ \mathrm{H} & 1.4315\end{array}$

$\begin{array}{rr}\mathrm{H} & 1.4315 \\ \mathrm{H} & -4.9112\end{array}$

$-7.3197$

$-3.1904$

0.6190

1.3314

1.8921

6.8953

6.6033

5.6794

0.4390

$\mathrm{N}-0.4735$

C -1.8435

C -2.3620

$\begin{array}{ll}0 & -2.2081\end{array}$

7.4072

6.9796

6.3470

6.9484

5.1343

$-3.4230$

$-3.0386$

4.4085

2. 9287

2.6401

1.1400

$-1.2985$

$-0.8018$

3. 1559

2. 6819

2. 3613

3. 5611

3.8892

3.7547

4. 3786

4.0657

2. 9291

2. 5161

2.9291
2.0809

1.4164

2.2006

7.9048

6.7538

6.2982

7.8478

2.4585

2. 4477

3.1595

2. 9420

2.6807

4. 2389

0.5585

0.7819

0.9162

4.9144

3.8289

5.2660

4.7045

2.6784

1.1904

2.0009
-5.3938

$-3.4311$

$-4.0380$

$-2.8145$

$-3.9588$

$-1.6387$

$-6.2351$

$-2.4279$

$-5.7528$

$-2.2369$

-6.5644
-4.6766

$-2.7118$

$-2.9560$

$-1.6841$

$-1.6542$

$-3.6385$

C -0.9187

$-3.1375$

$\begin{array}{lll}-1.2525 & -2.5553\end{array}$

$-0.8804$

$-4.4327$

$-1.3998-5.3964$

$-2.8845-5.0194$

$0.1693-1.7362$

-1.7362
-1.4592

$2.5582-2.0431$

$2.5820-3.1079$
$-3.1575$

2.9054

$-0.1225$

$-0.3495$

$-5.2212$

$-5.6508$

5.6904

5.8387

3.2268

4.0904

7. 1469

8.6491

6.5274

8.0258

8.0935

0.0535

$-1.0082$

$-2.1077$

$-5.6017$

$-4.8963$

4.8296

0.8812

$-2.5891$

$-1.9391$

$-1.1338$

$-2.3027$

$-2.0635$

$-3.3568$

$-4.4225$

$-4.3972$

$-4.3593$

$-4.3133$

$-4.1032$

$-5.5582$

2.4191

$-3.0418$

$-4.3963$

$-4.7568$

$-3.7772$

$-1.2370$

$-0.2270$

$-3.0053$

$-1.8528$

$-1.2128$

$-1.8242$

$-5.4558$

$-3.4457$

$-5.4948$

$-6.4677$

$-5.6697$

$-4.9541$

$-3.2067$

$-3.9914$

$-5.2768$

$-1.0219$

$-2.7392$

$-5.1562$

$-5.8038$

$-4.1049$

3. 3127

3.6986

4.0461

2.5012

1.0771

0.0957

0.8037

3.7112

4.1352

3.0011

1.8157

4.4595

3. 3807

3.2186

3.4004

2. 4988

3.0742 


\begin{tabular}{|c|c|c|c|}
\hline N & 3.6329 & -1.2710 & 8122 \\
\hline C & 4.9947 & -1.6189 & 1655 \\
\hline & 5.4637 & -0.9807 & 4868 \\
\hline & 6.6654 & -0.9290 & 7526 \\
\hline c & 5.9683 & -1.2371 & 3 \\
\hline C & 5.8487 & 0.1230 & 1.66 \\
\hline & 4.5009 & -0.5210 & 3128 \\
\hline & 4.8646 & -0.0079 & 5187 \\
\hline & -7.2802 & -2.7459 & 2.4927 \\
\hline H & -6.1529 & -1.4422 & 2.9688 \\
\hline $\mathrm{H}$ & -5.2876 & -4.3767 & 2.7760 \\
\hline 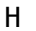 & -1.1541 & -4.7777 & 5.4618 \\
\hline & 0.2008 & -4.2841 & 4.4191 \\
\hline $\mathrm{H}$ & -1.2683 & -6.4491 & \\
\hline $\mathrm{H}$ & -0.8729 & -5.2085 & 2.44 \\
\hline ト & -3.5332 & -5.6756 & 3.81 \\
\hline ⺊ & -3.2008 & -5.0814 & 2.1718 \\
\hline ト & -1.7 & -2.4717 & \\
\hline $\mathrm{H}$ & 1.3799 & -0.3931 & 2.3083 \\
\hline $\mathrm{H}$ & 1.0552 & -1.9497 & 1.5467 \\
\hline $\mathrm{H}$ & 6.9782 & -1.3988 & 2.4221 \\
\hline $\mathrm{H}$ & 5.7 & -1.8954 & 1. \\
\hline $\mathrm{H}$ & 4.9789 & 95 & 1. \\
\hline $\mathrm{H}$ & 5.0319 & -2.7041 & 3.3192 \\
\hline H & 5.6270 & 0.7683 & 6.5126 \\
\hline $\mathrm{H}$ & 2.0497 & 6.1691 & -3 \\
\hline $\mathrm{H}$ & -4.5175 & 4.4886 & -4 \\
\hline $\mathrm{H}$ & -2.9181 & 4.6438 & -2 \\
\hline $\mathrm{H}$ & -0.3644 & 7.9519 & -3 \\
\hline $\mathrm{H}$ & 5.2795 & -0.7958 & 7. \\
\hline $\mathrm{H}$ & 691 & 0.2 & \\
\hline $\mathrm{H}$ & 3.5167 & -0.7153 & 89 \\
\hline $\mathrm{H}$ & 0.4137 & -1.6156 & 4.3955 \\
\hline $\mathrm{H}$ & -7.4939 & -3.0762 & 0. \\
\hline $\mathrm{H}$ & -6.3617 & -2.4233 & -0 \\
\hline $\mathrm{H}$ & 3.5028 & -0 . & 2 \\
\hline $\mathrm{H}$ & -5.9191 & -3.6495 & 4.2494 \\
\hline $\mathrm{C}$ & -10.7922 & -5.4489 & 1.3841 \\
\hline$C$ & -9.9500 & -4.5909 & 0. \\
\hline 0 & -8.9515 & -3.9997 & 0.8866 \\
\hline$C$ & -11.4998 & -4.5379 & 2.3933 \\
\hline $\mathrm{N}$ & -10.3945 & -4.4476 & -0.8033 \\
\hline $\mathrm{C}$ & -9.7475 & -3.5481 & -1 \\
\hline C & -9.4609 & 6.6747 & 2. \\
\hline C & -9.3063 & 5.4034 & 2. \\
\hline$C$ & -9.1971 & 5.6452 & 0.5372 \\
\hline $\mathrm{N}$ & -8.6654 & 4.5854 & -0 . \\
\hline 0 & -9.5940 & 6.6675 & -0 . \\
\hline $\mathrm{H}$ & -10.7630 & -3.9435 & 382 \\
\hline $\mathrm{H}$ & -12.0768 & -5.1239 & 3.1139 \\
\hline $\mathrm{H}$ & -12.1849 & -3.8502 & 1.8877 \\
\hline $\mathrm{H}$ & -11.5171 & -6.0488 & 0. \\
\hline $\mathrm{H}$ & -9.6318 & -2.5545 & -1 \\
\hline $\mathrm{H}$ & -8.4407 & 4.8186 & 2.3583 \\
\hline $\mathrm{H}$ & -10.1780 & 4.7531 & 2.1915 \\
\hline $\mathrm{H}$ & -8.1361 & 3.8777 & 0. \\
\hline $\mathrm{H}$ & -8.4678 & 4.7322 & -1 \\
\hline $\mathrm{H}$ & -10.2982 & 7.2741 & 2. \\
\hline $\mathrm{H}$ & -9.6315 & 6.4329 & 3.9192 \\
\hline $\mathrm{H}$ & -8.5629 & 7.2971 & 2.8 \\
\hline $\mathrm{H}$ & -10.1199 & -6.1374 & \\
\hline $\mathrm{H}$ & -10.3606 & -3.4678 & -2.6396 \\
\hline $\mathrm{H}$ & -8.7542 & -3.9138 & -2.0247 \\
\hline $\mathrm{H}$ & -11.2122 & -4.9590 & -1.0940 \\
\hline C & 6.4942 & -1.0316 & -3 \\
\hline$C$ & 5.8885 & -1.7603 & -2.4905 \\
\hline C & 5.0155 & -1.1359 & -1.6006 \\
\hline C & 4.7362 & 0.2299 & -1.7082 \\
\hline C & 5.3894 & 0.9643 & -2.709 \\
\hline C & 6.2466 & 0.3368 & -3.6121 \\
\hline $\mathrm{H}$ & 7.1937 & -1.5148 & -4.1858 \\
\hline $\mathrm{H}$ & 6.1444 & -2.8051 & -2.3586 \\
\hline $\mathrm{H}$ & 4.5539 & -1.6712 & -0.782 \\
\hline 11 & 5.2436 & 2.0342 & -2.7810 \\
\hline $\mathrm{H}$ & 6.7396 & 0.9262 & -4.3799 \\
\hline$C$ & 3.8227 & 0.8740 & -0.6559 \\
\hline 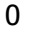 & 3.5816 & 0.0938 & 0.414 \\
\hline $\mathrm{H}$ & 7.2125 & 1.3389 & \\
\hline & & & \\
\hline
\end{tabular}

\section{TS2(BA)-S}

$\begin{array}{llll}C & 10.8915 & 5.5193 & -0.0376\end{array}$

$\begin{array}{llll}\text { C } & 9.4294 & 5.1581 & -0.3456\end{array}$

$\begin{array}{llll}C & 8.9365 & 3.9521 & 0.4048\end{array}$

$\begin{array}{llll}\mathrm{C} & 8.1141 & 2.9310 & -0.0252\end{array}$

$\begin{array}{llll}\mathrm{N} & 9.2454 & 3.7701 & 1.7404\end{array}$

$\begin{array}{llll}\text { C } & 8.6175 & 2.6647 & 2.1006\end{array}$

$\begin{array}{llll}\mathrm{N} & 7.9229 & 2.1098 & 1.0706\end{array}$

$\begin{array}{llll}H & 11.0213 & 5.6486 & 1.0392\end{array}$

$\begin{array}{llll}\text { H } & 9.2952 & 4.9934 & -1.4220\end{array}$

$\begin{array}{llll}H & 8.7976 & 6.0207 & -0.0888\end{array}$

$\begin{array}{llll}\mathrm{H} & 7.6512 & 2.7225 & -0.9781\end{array}$

$\begin{array}{lrrr}H & 8.6226 & 2.2242 & 3.0872\end{array}$

C $\quad-9.6209 \quad 1.4886 \quad-4.8966$

$\begin{array}{llll}\text { C } & -8.7058 & 2.2826 & -3.9455\end{array}$

$\begin{array}{llll}C & -7.6083 & 1.4497 & -3.3156\end{array}$

$\begin{array}{llll}C & -6.5104 & 1.0108 & -4.0730\end{array}$

$\begin{array}{llll}\text { C } & -7.6414 & 1.0913 & -1.9639\end{array}$

$\begin{array}{llll}C & -5.4983 & 0.2407 & -3.5103\end{array}$

$\begin{array}{llll}\text { C } & -6.6286 & 0.3283 & -1.3757\end{array}$

$\begin{array}{llll}\text { C } & -5.5459 & -0.1046 & -2.1517\end{array}$

$\begin{array}{llll}0 & -4.5105 & -0.8367 & -1.6627\end{array}$

$\begin{array}{lll}\mathrm{H}-10.1226 & 0.6754 & -4.3620\end{array}$

$\begin{array}{llll}H & -9.3122 & 2.7355 & -3.1528\end{array}$

$\begin{array}{llll}\mathrm{H} & -8.2572 & 3.1147 & -4.5038\end{array}$

$\begin{array}{llll}\mathrm{H} & -6.4446 & 1.2821 & -5.1243\end{array}$

$\begin{array}{llll}\mathrm{H} & -8.4560 & 1.4452 & -1.3369\end{array}$

$\begin{array}{llll}H & -4.6513 & -0.0935 & -4.0997\end{array}$

$\begin{array}{llll}H & -6.6461 & 0.1421 & -0.3074\end{array}$

$\begin{array}{llll}\mathrm{H} & -4.6330 & -1.1349 & -0.7272\end{array}$

$\begin{array}{llll}\text { C } & -0.0679 & -6.9695 & -4.9744\end{array}$

$\begin{array}{llll}\text { C } & -0.2310 & -6.5196 & -3.5172\end{array}$

$\begin{array}{llll}\text { C } & -1.5475 & -5.8572 & -3.1491\end{array}$

C $\quad-1.9799 \quad-5.8664-1.8150$

C $\quad-2.3463 \quad-5.1731-4.0759$

$\begin{array}{llll}\text { C } & -3.1482 & -5.2224 & -1.4147\end{array}$

$\begin{array}{llll}\text { C } & -3.5227 & -4.5286 & -3.6943\end{array}$

$\begin{array}{llll}\text { C } & -3.9344 & -4.5519 & -2.3584\end{array}$

$\begin{array}{llll}0 & -5.1131 & -3.9456 & -2.0418\end{array}$

H $\quad-0.0804 \quad-6.1197 \quad-5.6645$

H $\quad-0.0833 \quad-7.3820 \quad-2.8564$

H $\quad 0.5902-5.8306-3.2763$

$\begin{array}{llll}\mathrm{H} & -1.3864 & -6.3845 & -1.0676\end{array}$

H $-2.0509-5.1373-5.1199$

H $\quad-3.4525 \quad-5.2422-0.3716$

H $\quad-4.1332 \quad-3.9999-4.4183$

$\begin{array}{llll}\mathrm{H} & -5.2064 & -3.8712 & -1.0805\end{array}$

$\begin{array}{lll}5.0734 & -4.3602 & -5.1716\end{array}$

$\begin{array}{lll}5.2637 & -5.1579 & -3.9009\end{array}$

$6.1959-5.9391-3.7272$

$\begin{array}{lll}6.3726 & -4.1919 & -5.9575\end{array}$

$\begin{array}{lll}4.3327 & -4.8943 & -5.7824\end{array}$

$6.8281 \quad-5.1671-6.1416$

$\begin{array}{lll}7.0990 & -3.5943 & -5.3986\end{array}$

$\begin{array}{llll}6.1879 & -3.6970 & -6.9152\end{array}$

$\begin{array}{lll}4.2642 & -4.9820 & -2.9951\end{array}$

$\begin{array}{lll}4.2993 & -5.6559 & -1.7174\end{array}$

$\begin{array}{lll}2.9132 & -5.7097 & -1.0778\end{array}$

$2.3832-4.3530-0.5836$

$\begin{array}{lll}0.8906 & -4.4370 & -0.2533\end{array}$

$\begin{array}{lll}3.1699 & -3.8629 & 0.6303\end{array}$

$\begin{array}{lll}3.7035 & -4.1467 & -3.0732\end{array}$

$\begin{array}{lll}5.0245 & -5.1862 & -1.0396\end{array}$

$2.9504-6.3948-0.2213$

$\begin{array}{lll}2.2162 & -6.1502 & -1.8010\end{array}$

$2.5035-3.6073-1.3852$

$\begin{array}{lll}0.7048 & -5.2643 & 0.4424\end{array}$

$\begin{array}{lll}0.5223 & -3.5274 & 0.2277\end{array}$

$0.2864-4.6144-1.1463$

$\begin{array}{lll}4.2514 & -3.8792 & 0.4664\end{array}$

$\begin{array}{lll}2.9532 & -4.4648 & 1.5166\end{array}$

$\begin{array}{lll}2.8971 & -2.8374 & 0.8707\end{array}$

$\begin{array}{lll}9.1525 & -5.2627 & -0.2205\end{array}$

$\begin{array}{lll}7.9964 & -4.4720 & -0.8524\end{array}$

$8.4720-3.2021-1.5184$

$8.9222-3.2267 \quad-2.8444$

$8.4921-1.9849-0.8266$

$\begin{array}{lll}9.3724 & -2.0609 & -3.4650\end{array}$ $\begin{array}{llll}\text { C } & 8.9335 & -0.8152 & -1.4436\end{array}$

$\begin{array}{llll}C & 9.3743 & -0.8515 & -2.7673\end{array}$

$\begin{array}{llll}\mathrm{H} & 9.6674 & -4.6626 & 0.5361\end{array}$

H $\quad 7.2626-4.2211-0.0763$

H $\quad 7.4868 \quad-5.1026 \quad-1.5896$

$\begin{array}{llll}\text { H } & 8.8913 & -4.1636 & -3.3952\end{array}$

$\begin{array}{llll}\text { H } & 8.1381 & -1.9487 & 0.1995\end{array}$

H $\quad 9.7119-2.0950-4.4969$

$\begin{array}{llll}\mathrm{H} & 8.9240 & 0.1220 & -0.8951\end{array}$

$\begin{array}{llll}H & 9.7134 & 0.0592 & -3.2522\end{array}$

$\begin{array}{llll}\text { C } & -2.8166 & 0.8592 & 0.4540\end{array}$

$\begin{array}{llll}\text { C } & -1.6050 & 1.2890 & -0.0301\end{array}$

$\begin{array}{llll}C & -1.5864 & 2.6158 & -0.5837\end{array}$

$\begin{array}{llll}\mathrm{N} & -0.4588 & 3.2585 & -0.8320\end{array}$

$\begin{array}{llll}\mathrm{N} & -2.7594 & 3.2531 & -0.8735\end{array}$

$\begin{array}{llll}\text { C } & -3.8948 & 2.7461 & -0.4294\end{array}$

$\begin{array}{llll}\mathrm{N} & -3.9455 & 1.6061 & 0.3043\end{array}$

$\begin{array}{llll}\mathrm{S} & 2.0022 & -0.5991 & -2.8631\end{array}$

$\begin{array}{llll}C & 1.4957 & 0.4562 & -1.6039\end{array}$

$\begin{array}{llll}C & -5.1736 & 3.4347 & -0.7631\end{array}$

$\begin{array}{llll}\mathrm{N} & 0.3441 & -0.0032 & -1.0595\end{array}$

$\begin{array}{llll}\text { C } & -0.1793 & -1.1673 & -1.6681\end{array}$

$\begin{array}{llll}\text { C } & -1.4851 & -1.7569 & -1.2536\end{array}$

$\begin{array}{llll}\text { C } & 0.6143 & -1.6179 & -2.6793\end{array}$

C $\quad 0.4162-2.7792-3.6000$

$\begin{array}{llll}C & -0.3373 & 0.5052 & 0.1947\end{array}$

$\begin{array}{llll}\mathrm{H} & 0.4558 & 2.9101 & -0.4768\end{array}$

$\begin{array}{llll}H & -0.4909 & 4.2526 & -1.0623\end{array}$

$\begin{array}{llll}H & -0.5670 & -0.3861 & 0.7772\end{array}$

$\begin{array}{rrrr}\mathrm{H} & 0.4060 & 1.0987 & 0.7197\end{array}$

$\begin{array}{llll}H & -5.8816 & 3.3559 & 0.0694\end{array}$

$\begin{array}{llll}\mathrm{H} & -4.9752 & 4.4768 & -1.0136\end{array}$

$\begin{array}{llll}H & -5.6304 & 2.9404 & -1.6278\end{array}$

$\begin{array}{llll}H & -2.9403 & -0.0789 & 0.9883\end{array}$

$\begin{array}{llll}\mathrm{H} & -1.5284 & -1.9699 & -0.1839\end{array}$

H $\quad-1.6375-2.7007-1.7760$

H $\quad-2.3174-1.1029-1.5218$

$\begin{array}{llll}\mathrm{H} & 1.2891 & -3.4406 & -3.5999\end{array}$

H $\quad 0.2530 \quad-2.4411-4.6289$

$\begin{array}{llll}\text { C } & 1.2451 & -2.8644 & 7.5708\end{array}$

$\begin{array}{llll}\text { C } & 0.5644 & -1.4955 & 7.6489\end{array}$

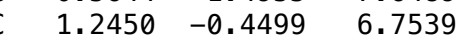

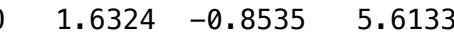

$\begin{array}{llll}0 & 1.3506 & 0.7286 & 7.1876\end{array}$

$\begin{array}{llll}\text { C } & -8.9339 & 2.2016 & 3.6259\end{array}$

$\begin{array}{llll}C & -7.8488 & 1.2027 & 3.2005\end{array}$

$\begin{array}{llll}\text { C } & -6.9490 & 1.6616 & 2.0419\end{array}$

$\begin{array}{llll}0 & -7.1611 & 2.7822 & 1.5208\end{array}$

$\begin{array}{llll}0 & -6.0262 & 0.8445 & 1.6963\end{array}$

$\begin{array}{llll}\mathrm{H} & -9.5194 & 1.7989 & 4.4597\end{array}$

$\begin{array}{llll}\mathrm{H} & -8.4915 & 3.1480 & 3.9438\end{array}$

$\begin{array}{llll}\mathrm{H} & -8.2944 & 0.2443 & 2.9035\end{array}$

$\begin{array}{llll}\mathrm{H} & -7.1844 & 0.9636 & 4.0397\end{array}$

$\begin{array}{llll}\text { C } & -1.4953 & 1.7631 & 5.4267\end{array}$

$\begin{array}{llll}\text { C } & -0.8579 & 3.0318 & 4.8457\end{array}$

$\begin{array}{llll}C & 0.4460 & 2.7446 & 4.1791\end{array}$

$\begin{array}{llll}\text { C } & 0.8775 & 2.9327 & 2.8833\end{array}$

N $\quad 1.4867 \quad 2.1051 \quad 4.8195$

C $2.4733 \quad 1.9220 \quad 3.9065$

$\begin{array}{llll}\mathrm{N} & 2.1483 & 2.4160 & 2.7207\end{array}$

$\mathrm{H}-1.6086 \quad 0.9944 \quad 4.6555$

$\begin{array}{llll}\mathrm{H} & -1.5238 & 3.4901 & 4.1059\end{array}$

$\begin{array}{llll}\mathrm{H} & -0.7270 & 3.7717 & 5.6474\end{array}$

$\begin{array}{llll}\mathrm{H} & 0.3582 & 3.4213 & 2.0699\end{array}$

$\begin{array}{llll}\mathrm{H} & 3.3941 & 1.4127 & 4.1423\end{array}$

$\begin{array}{llll}\mathrm{H} & 2.6172 & 1.6269 & 0.7354\end{array}$

$\begin{array}{lll}\text { C } & 4.6835 & 7.1298 \quad 3.1763\end{array}$

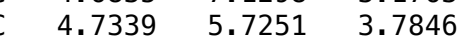

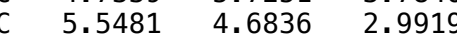

$\begin{array}{llll}\text { C } & 5.7206 & 3.4215 & 3.8512\end{array}$

$\begin{array}{llll}\text { C } & 4.8999 & 4.3395 & 1.6417\end{array}$

$\mathrm{H} \quad 4.1966$

$\begin{array}{llll}\mathrm{H} & 3.7122 & 5.3404 & 3.9067\end{array}$

$\begin{array}{llll}\mathrm{H} & 5.1556 & 5.7958 & 4.7966\end{array}$

H $\quad 6.5465 \quad 5.1001 \quad 2.7929$

$\begin{array}{llll}\mathrm{H} & 4.7612 & 3.1270 & 4.2839\end{array}$

$\begin{array}{llll}H & 6.0896 & 2.5730 & 3.2707\end{array}$

$\begin{array}{llll}\mathrm{H} & 6.4180 & 3.5997 & 4.6777\end{array}$

$\begin{array}{llll}H & 3.9348 & 3.8424 & 1.7869\end{array}$

$\begin{array}{llll}\mathrm{H} & 5.5508 & 3.6651 & 1.0754\end{array}$

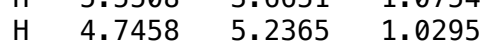




\begin{tabular}{|c|c|c|c|}
\hline $\mathrm{H}$ & -0.4488 & -3.3711 & -3.3037 \\
\hline $\mathrm{H}$ & -9.6138 & 2.4145 & 2.7991 \\
\hline $\mathrm{H}$ & 11.5677 & 4.7197 & -0.3577 \\
\hline $\mathrm{H}$ & 11.1846 & 6.4441 & -0.5459 \\
\hline H & -10.3873 & 2.1350 & -5.3361 \\
\hline $\mathrm{H}$ & -9.0430 & 1.0416 & -5.7122 \\
\hline ト & -0.8713 & 1.3529 & 6.2251 \\
\hline H & -2.4864 & 1.9741 & 5.8422 \\
\hline $\mathrm{H}$ & 5.6926 & 7.5382 & 3.0440 \\
\hline $\mathrm{H}$ & 4.1248 & 7.8193 & 3.8185 \\
\hline $\mathrm{H}$ & -0.4778 & -1.5772 & 7.3051 \\
\hline $\mathrm{H}$ & 0.5265 & -1.1127 & 8.6726 \\
\hline H & 1.3610 & -3.1665 & 6.5272 \\
\hline H & 0.6764 & -3.6313 & 8.1090 \\
\hline $\mathrm{H}$ & 2.2492 & -2.8236 & 8.0065 \\
\hline $\mathrm{H}$ & 8.7896 & -6.1791 & 0.2570 \\
\hline $\mathrm{H}$ & 9.8880 & -5.5431 & -0.9811 \\
\hline $\mathrm{H}$ & 4.6770 & -6.6639 & -1.9043 \\
\hline $\mathrm{H}$ & 0.8882 & -7.4835 & -5.1061 \\
\hline $\mathrm{H}$ & -0.8697 & -7.6526 & -5.2719 \\
\hline $\mathrm{H}$ & 4.6330 & -3.3851 & -4.9347 \\
\hline $\mathrm{H}$ & 1.4768 & 1.6733 & 5.7712 \\
\hline $\mathrm{H}$ & -4.8721 & 1.3134 & 0.8310 \\
\hline $\mathrm{C}$ & 1.7015 & 6.7263 & -2.8023 \\
\hline $\mathrm{C}$ & 0.3536 & 6.6125 & -2.1383 \\
\hline 0 & 0.1884 & 5.9197 & -1.1319 \\
\hline $\mathrm{N}$ & -0.6653 & 7.2930 & -2.7319 \\
\hline C & -2.0467 & 6.9173 & -2.4745 \\
\hline C & -2.5383 & 6.1448 & -3.6984 \\
\hline 0 & -2.3676 & 6.6274 & -4.8213 \\
\hline $\mathrm{N}$ & -3.1136 & 4.9500 & -3.4547 \\
\hline $\mathrm{C}$ & -3.5666 & 4.0963 & -4.5439 \\
\hline C & -3.0592 & 2.6631 & -4.4041 \\
\hline $\mathrm{C}$ & -1.5351 & 2.5037 & -4.3296 \\
\hline C & -1.1998 & 1.0186 & -4.1719 \\
\hline $\mathrm{C}$ & -0.8219 & 3.1193 & -5.5384 \\
\hline $\mathrm{C}$ & 2.6960 & 2.6423 & -2.3059 \\
\hline $\mathrm{C}$ & 3.4447 & 3.7602 & -1.8926 \\
\hline $\mathrm{C}$ & 4.0852 & 4.5716 & -2.8208 \\
\hline$C$ & 3.9272 & 4.3414 & -4.1910 \\
\hline C & 3.0957 & 3.3074 & -4.6106 \\
\hline $\mathrm{C}$ & 2.4925 & 2.4603 & -3.6746 \\
\hline $\mathrm{C}$ & 2.3700 & 1.6437 & -1.1898 \\
\hline 0 & 1.8916 & 2.2400 & -0.0485 \\
\hline $\mathrm{H}$ & 1.8013 & 7.6323 & -3.4070 \\
\hline $\mathrm{H}$ & 2.4778 & 6.6992 & -2.0398 \\
\hline $\mathrm{H}$ & -2.0774 & 6.3397 & -1.5505 \\
\hline $\mathrm{H}$ & -2.6672 & 7.8115 & -2.3553 \\
\hline $\mathrm{H}$ & -3.5105 & 2.2084 & -3.5153 \\
\hline $\mathrm{H}$ & -3.4419 & 2.0897 & -5.2599 \\
\hline $\mathrm{H}$ & -1.1777 & 3.0223 & -3.4319 \\
\hline $\mathrm{H}$ & 0.2603 & 2.9800 & -5.4609 \\
\hline $\mathrm{H}$ & -1.1575 & 2.6500 & -6.4721 \\
\hline $\mathrm{H}$ & -1.0101 & 4.1942 & -5.6145 \\
\hline $\mathrm{H}$ & -1.5321 & 0.4457 & -5.0468 \\
\hline $\mathrm{H}$ & -1.6942 & 0.5942 & -3.2929 \\
\hline $\mathrm{H}$ & -0.1247 & 0.8622 & -4.0641 \\
\hline $\mathrm{H}$ & -3.2294 & 4.5636 & -5.4699 \\
\hline $\mathrm{H}$ & 3.5405 & 3.9601 & -0.8319 \\
\hline $\mathrm{H}$ & 4.7008 & 5.3953 & -2.4724 \\
\hline $\mathrm{H}$ & 4.4287 & 4.9741 & -4.9170 \\
\hline $\mathrm{H}$ & 2.9281 & 3.1330 & -5.6692 \\
\hline $\mathrm{H}$ & 1.8882 & 1.6436 & -4.0420 \\
\hline C & -5.3731 & -3.6042 & 3.3443 \\
\hline $\mathrm{C}$ & -4.0464 & -2.9474 & 3.7644 \\
\hline 0 & -4.0506 & -1.8329 & 4.2800 \\
\hline$C$ & -6.2434 & -2.5997 & 2.5608 \\
\hline C & -5.8012 & -2.3470 & 1.1305 \\
\hline $\mathrm{N}$ & -6.7269 & -2.6106 & 0.1714 \\
\hline 0 & -4.6680 & -1.9363 & 0.8318 \\
\hline $\mathrm{N}$ & -2.9017 & -3.6669 & 3.6137 \\
\hline $\mathrm{C}$ & -1.6235 & -3.1468 & 4.0859 \\
\hline C & -0.9024 & -2.3028 & 3.0266 \\
\hline 0 & -1.1975 & -2.3870 & 1.8233 \\
\hline C & -0.7996 & -4.4343 & 4.3014 \\
\hline $\mathrm{C}$ & -1.2598 & -5.3101 & 3.1234 \\
\hline C & -2.7526 & -4.9645 & 2.9457 \\
\hline $\mathrm{N}$ & 0.1300 & -1.5916 & 3.4972 \\
\hline $\mathrm{C}$ & 1.2284 & -1.1797 & 2.6596 \\
\hline C & 2.5125 & -1.8964 & 3.0965 \\
\hline & & & \\
\hline
\end{tabular}

\section{N $\quad 3.6211 \quad-1.1890 \quad 2.7779$ \\ $\begin{array}{llll}\text { C } & 4.9736 & -1.5697 & 3.1300\end{array}$ \\ Int2(BA)-R}

$\begin{array}{llll}\text { C } & 5.4360 & -0.9871 & 4.4812\end{array}$

$\begin{array}{llll}\mathrm{C} & 6.6323 & -0.9915 & 4.7742 \\ \mathrm{C} & 5.9687 & -1.1426 & 2.0444\end{array}$

$\begin{array}{rrrr}0 & 5.9285 & 0.2543 & 1.7871\end{array}$

$\begin{array}{llll}\mathrm{N} & 4.4720 & -0.4806 & 5.2738\end{array}$

$\begin{array}{llll}\text { C } & 4.8211 & 0.0921 & 6.5534\end{array}$

$\begin{array}{llll}\text { H } & -7.2869 & -2.9229 & 2.5651\end{array}$

$\begin{array}{llll}\text { H } & -6.1714 & -1.6261 & 3.0579\end{array}$

$\begin{array}{llll}H & -5.2337 & -4.5282 & 2.7777\end{array}$

H $\quad-1.0763 \quad-4.8840 \quad 5.2604$

H $\quad 0.2752-4.2400 \quad 4.2944$

H $\quad-1.1026-6.3780 \quad 3.2954$

$\begin{array}{llll}\mathrm{H} & -0.7120 & -5.0228 & 2.2240\end{array}$

H $\quad-3.3971 \quad-5.7173 \quad 3.4160$

$\begin{array}{llll}\mathrm{H} & -3.0221 & -4.8867 & 1.8869\end{array}$

$\begin{array}{llll}\mathrm{H} & -1.7722 & -2.5617 & 4.9954\end{array}$

$\begin{array}{llll}H & 1.3697 & -0.0973 & 2.6917\end{array}$

$\begin{array}{llll}\mathrm{H} & 1.0123 & -1.4751 & 1.6305\end{array}$

$\begin{array}{llll}\text { H } & 6.9690 & -1.3678 & 2.4184\end{array}$

$\begin{array}{llll}\mathrm{H} & 5.7872 & -1.7177 & 1.1290\end{array}$

$\begin{array}{llll}\mathrm{H} & 5.0505 & 0.5078 & 1.4021\end{array}$

$\begin{array}{llll}H & 4.9966 & -2.6612 & 3.2252\end{array}$

$\begin{array}{llll}\mathrm{H} & 5.5914 & 0.8574 & 6.4230\end{array}$

$\begin{array}{llll}\mathrm{H} & 1.8397 & 5.8564 & -3.4483\end{array}$

H $\quad-4.6648 \quad 4.0821 \quad-4.5656$

$\begin{array}{llll}\mathrm{H} & -3.0624 & 4.5505 & -2.5169\end{array}$

$\begin{array}{llll}\mathrm{H} & -0.5132 & 7.6524 & -3.6664\end{array}$

$\begin{array}{lll}5.2181 & -0.6626 & 7.2443\end{array}$

$\begin{array}{lll}3.9242 & 0.5394 & 6.9849\end{array}$

$\begin{array}{lll}3.4786 & -0.6576 \quad 5.1189\end{array}$

$0.3820-1.5585 \quad 4.4936$

$\begin{array}{llll}\mathrm{H} & -7.6881 & -2.8413 & 0.4242\end{array}$

$\begin{array}{llll}\mathrm{H} & -6.5741 & -2.2182 & -0.7487\end{array}$

$\begin{array}{llll}H & 3.4890 & -0.3135 & 2.2831\end{array}$

$\begin{array}{llll}\mathrm{H} & -5.8964 & -3.8609 & 4.2713\end{array}$

$\begin{array}{lll}\text { C }-10.3502 & -5.5593 & 1.6767\end{array}$

$\begin{array}{llll}\text { C } & -9.8075 & -4.5827 & 0.6579\end{array}$

$\begin{array}{llll}0 & -9.2405 & -3.5449 & 1.0170\end{array}$

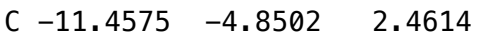

$\begin{array}{lll}\mathrm{N}-10.0651 & -4.8551 & -0.6399\end{array}$

$\begin{array}{llll}\text { C } & -9.7308 & -3.9202 & -1.6978\end{array}$

$\begin{array}{llll}\text { C } & -9.6374 & 6.4077 & 2.6770\end{array}$

$\begin{array}{llll}\text { C } & -9.4637 & 5.1348 & 1.8569\end{array}$

$\begin{array}{llll}C & -9.3262 & 5.3783 & 0.3563\end{array}$

$\begin{array}{llll}\mathrm{N} & -8.7351 & 4.3405 & -0.3097\end{array}$

$\begin{array}{llll}0 & -9.7507 & 6.3829 & -0.2067\end{array}$

H $-11.0640 \quad-3.9372 \quad 2.9131$

$\begin{array}{lll}\mathrm{H}-11.8511 & -5.4918 & 3.2547\end{array}$

H $-12.2877-4.5747 \quad 1.8036$

$\begin{array}{lll}\mathrm{H}-10.7244 & -6.4742 & 1.2040\end{array}$

$\begin{array}{llll}\mathrm{H} & -9.8687 & -2.9022 & -1.3288\end{array}$

$\begin{array}{llll}\mathrm{H} & -8.6030 & 4.5532 & 2.1927\end{array}$

$\begin{array}{lll}\mathrm{H}-10.3374 & 4.4829 & 1.9910\end{array}$

$\begin{array}{llll}\mathrm{H} & -8.1926 & 3.6537 & 0.2129\end{array}$

$\begin{array}{llll}\mathrm{H} & -8.5217 & 4.4929 & -1.2843\end{array}$

$\begin{array}{lll}\mathrm{H}-10.4648 & 7.0072 & 2.2887\end{array}$

$\begin{array}{llll}\mathrm{H} & -9.8339 & 6.1679 & 3.7265\end{array}$

$\begin{array}{llll}H & -8.7369 & 7.0286 & 2.6341\end{array}$

$\begin{array}{llll}\mathrm{H} & -9.5305 & -5.8377 & 2.3459\end{array}$

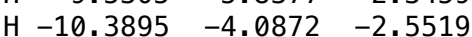

$\begin{array}{llll}\mathrm{H} & -8.6904 & -4.0294 & -2.0266\end{array}$

H $-10.5080-5.7290-0.8752$

$\begin{array}{lrrr}\text { C } \quad 5.9017 & -1.7175 & -3.0830\end{array}$

$\begin{array}{llll}\text { C } & 5.2803 & -2.1359 & -1.9069\end{array}$

$\begin{array}{llll}\text { C } & 4.5968 & -1.2214 & -1.1132\end{array}$

$\begin{array}{llll}C & 4.5616 & 0.1271 & -1.4662\end{array}$

$\begin{array}{llll}\text { C } & 5.2171 & 0.5524 & -2.6307\end{array}$

$\begin{array}{llll}\text { C } & 5.8691 & -0.3711 & -3.4443\end{array}$

H $\quad 6.4665 \quad-2.4246 \quad-3.6739$

H $\quad 5.3587-3.1680-1.5962$

H $\quad 4.0934-1.5239-0.2066$

$\begin{array}{llll}\mathrm{H} & 5.2146 & 1.6052 & -2.8935\end{array}$

H $\quad 6.3840 \quad-0.0383-4.3400$

$\begin{array}{llll}\text { C } & 3.8341 & 1.1176 & -0.6193\end{array}$

$\begin{array}{llll}0 & 3.5289 & 0.7528 & 0.6680\end{array}$

$\begin{array}{llll}\mathrm{H} & 7.2624 & 1.3293 & 1.1681\end{array}$

$\begin{array}{llll}\mathrm{H} & 4.4141 & 2.0516 & -0.6384\end{array}$

\begin{tabular}{|c|c|c|c|}
\hline & 021 & 117 & -0.2044 \\
\hline & 11.0538 & 000 & -0.451 \\
\hline & 9.9645 & & a 25 \\
\hline & & & -6 \\
\hline & 10.0054 & & \\
\hline & 8.9103 & 343 & 110 \\
\hline & & & 043 \\
\hline & 10.2 & & -0. \\
\hline & 12. & & -0 \\
\hline & 10. & & -1.51 \\
\hline & & & \\
\hline & 8 & & \\
\hline & 7.2 & & 06 \\
\hline & -9.7 & & -4 \\
\hline & -8. & & -3 \\
\hline & -7 . & & \\
\hline & -6.6 & & -3 \\
\hline & -7 & & -1 \\
\hline & -5 . & & \\
\hline & -6 & & \\
\hline & -5.8480 & 0. & -2.0974 \\
\hline & -5 & & \\
\hline & -10. & & \\
\hline & -8.8 & & -2 . \\
\hline & -8 & & -3 \\
\hline & -6 . & & \\
\hline & -8 & & \\
\hline & -5.7 & & -4 \\
\hline & -6. & & -0 \\
\hline & -4 . & & \\
\hline & -0. & -6 & -5 \\
\hline & -0.7 & -6 . & -4 \\
\hline & -2 & & \\
\hline & -2 . & & \\
\hline & -3. & -5 & -4 \\
\hline$c$ & -3.2 & -5 . & -1 \\
\hline & -4 . & & \\
\hline & -4 . & & \\
\hline & -5 & -4 & -1 \\
\hline & -0.8 & & -6 \\
\hline & -0 & & \\
\hline & & & \\
\hline & -1 & -5 . & -1 \\
\hline & -3. & -5 & -5 \\
\hline & & & \\
\hline & -5. & & -4 \\
\hline & -5.7 & -4 . & -1 \\
\hline & 4. & -3 & -6 \\
\hline & & & \\
\hline ) & 6. & -3 & -4 \\
\hline$C$ & 5.3 & -2 & -6 . \\
\hline & & & -6 \\
\hline & & & \\
\hline & & & -5 \\
\hline & & & -7 \\
\hline & & & \\
\hline & & & \\
\hline & & & -1 \\
\hline & 2.3 & & -1 \\
\hline & & & \\
\hline & & & \\
\hline & & & -4 \\
\hline & & -4 & -2 . \\
\hline & & & \\
\hline & & & \\
\hline & & & -2 \\
\hline & & & \\
\hline$\pi$ & & & \\
\hline & & & \\
\hline & & & -0 \\
\hline & & & \\
\hline & & & \\
\hline & & & -1 \\
\hline & & & \\
\hline & & & \\
\hline & & & \\
\hline & & & \\
\hline
\end{tabular}




\begin{tabular}{|c|c|c|c|c|c|c|c|}
\hline C & 9.4221 & -0.9472 & -0.2619 & $\mathrm{H}$ & 5.2069 & 5.0261 & 1.3842 \\
\hline$C$ & 9.2112 & -2.1729 & 1.8055 & $\mathrm{H}$ & -1.8247 & -1.9031 & -4.9923 \\
\hline C & 9.6125 & -1.0255 & 1.1193 & $\mathrm{H}$ & -8.9004 & 1.3265 & 4.7088 \\
\hline $\mathrm{H}$ & 9.5495 & -5.6948 & -0.6912 & $\mathrm{H}$ & 11.3736 & 5.4995 & 0.8591 \\
\hline $\mathrm{H}$ & 7.1745 & -4.8986 & -0.3968 & $\mathrm{H}$ & 12.0422 & 5.7265 & -0.7733 \\
\hline $\mathrm{H}$ & 7.2945 & -3.9931 & -1.9050 & $\mathrm{H}$ & -10.4618 & 3.4241 & -4.3148 \\
\hline $\mathrm{H}$ & 8.6813 & -1.9362 & -2.0221 & $\mathrm{H}$ & -9.4365 & 2.0942 & -4.8897 \\
\hline $\mathrm{H}$ & 8.3075 & -4.1202 & 1.6506 & $\mathrm{H}$ & -0.9174 & 2.4564 & 5.8430 \\
\hline $\mathrm{H}$ & 9.7078 & -0.0445 & -0.7917 & $\mathrm{H}$ & -1.8601 & 0.9754 & 6.1047 \\
\hline $\mathrm{H}$ & 9.2936 & -2.2163 & 2.8852 & $\mathrm{H}$ & 6.3354 & 7.0349 & 3.3769 \\
\hline $\mathrm{H}$ & 10.0378 & -0.1840 & 1.6558 & $\mathrm{H}$ & 4.9133 & 7.4804 & 4.3332 \\
\hline C & -2.7579 & 0.6760 & 0.8537 & $\mathrm{H}$ & -0.0296 & -2.3349 & 6.7883 \\
\hline C & -1.6610 & 1.0727 & 0.1360 & $\mathrm{H}$ & 0.8353 & -2.0337 & 8.3032 \\
\hline C & -1.7232 & 2.3853 & -0.4641 & $\mathrm{H}$ & 1.9418 & -3.7292 & 5.9805 \\
\hline $\mathrm{N}$ & -0.6919 & 2.9207 & -1.0767 & $\mathrm{H}$ & 1.1613 & -4.4321 & 7.4203 \\
\hline $\mathrm{N}$ & -2.8784 & 3.1212 & -0.3959 & $\mathrm{H}$ & 2.6877 & -3.5465 & 7.5677 \\
\hline C & -3.9008 & 2.6585 & 0.2911 & $\mathrm{H}$ & 8.5474 & -6.1406 & -2.0900 \\
\hline $\mathrm{N}$ & -3.8664 & 1.4646 & 0.9316 & $\mathrm{H}$ & 9.6675 & -4.7703 & -2.1932 \\
\hline $\mathrm{S}$ & 0.6989 & -0.7321 & -3.6745 & $\mathrm{H}$ & 4.3289 & -6.3716 & -2.8771 \\
\hline C & 0.8354 & 0.0747 & -2.1639 & $\mathrm{H}$ & 0.4037 & -6.3230 & -6.0833 \\
\hline C & -5.1298 & 3.4964 & 0.4223 & $\mathrm{H}$ & -1.2895 & -6.7755 & -6.2829 \\
\hline $\mathrm{N}$ & -0.1725 & -0.2951 & -1.3563 & $\mathrm{H}$ & 3.5307 & -3.5296 & -6.1688 \\
\hline C & -1.0579 & -1.2563 & -1.8997 & $\mathrm{H}$ & 1.8019 & 0.3346 & 4.5701 \\
\hline C & -2.1595 & -1.8389 & -1.0926 & $\mathrm{H}$ & -4.7426 & 1.1374 & 1.4587 \\
\hline C & -0.7334 & -1.5805 & -3.1794 & C & 1.9099 & 7.1790 & -2.1313 \\
\hline C & -1.4506 & -2.4758 & -4.1369 & C & 0.6052 & 6.5121 & -1.7653 \\
\hline C & -0.4208 & 0.2163 & 0.0399 & 0 & 0.3763 & 5.3369 & -2.0592 \\
\hline $\mathrm{H}$ & 0.3021 & 2.5061 & -1.0301 & $\mathrm{~N}$ & -0.3132 & 7.2631 & -1.1016 \\
\hline $\mathrm{H}$ & -0.7480 & 3.8759 & -1.4197 & C & -1.6697 & 6.7530 & -0.9987 \\
\hline $\mathrm{H}$ & -0.5304 & -0.6651 & 0.6697 & C & -2.2475 & 6.4976 & -2.4028 \\
\hline $\mathrm{H}$ & 0.4666 & 0.7711 & 0.3318 & 0 & -2.0048 & 7.2783 & -3.3207 \\
\hline $\mathrm{H}$ & -6.0349 & 2.8911 & 0.4104 & $\mathrm{~N}$ & -3.0266 & 5.3983 & -2.5045 \\
\hline $\mathrm{H}$ & -5.1077 & 4.0320 & 1.3779 & C & -3.5771 & 4.9732 & -3.7838 \\
\hline $\mathrm{H}$ & -5.1538 & 4.2339 & -0.3795 & C & -3.0430 & 3.6082 & -4.2273 \\
\hline $\mathrm{H}$ & -2.8268 & -0.2683 & 1.3832 & C & -1.5323 & 3.5475 & -4.5003 \\
\hline $\mathrm{H}$ & -1.8093 & -2.2017 & -0.1232 & C & -1.1194 & 2.1024 & -4.7904 \\
\hline $\mathrm{H}$ & -2.5917 & -2.6821 & -1.6271 & C & -1.1028 & 4.4908 & -5.6298 \\
\hline $\mathrm{H}$ & -2.9508 & -1.1144 & -0.9404 & C & 2.4514 & 1.6886 & -3.1958 \\
\hline $\mathrm{H}$ & -2.3022 & -2.9578 & -3.6601 & C & 2.2055 & 3.0551 & -3.3482 \\
\hline $\mathrm{H}$ & -0.7932 & -3.2609 & -4.5210 & C & 2.5294 & 3.7104 & -4.5362 \\
\hline C & 1.7273 & -3.5735 & 7.0416 & C & 3.1091 & 3.0103 & -5.5936 \\
\hline C & 0.9686 & -2.2572 & 7.2414 & C & 3.3730 & 1.6477 & -5.4459 \\
\hline C & 1.7007 & -1.0812 & 6.5788 & C & 3.0513 & 0.9952 & -4.2577 \\
\hline 0 & 1.8416 & -1.1901 & 5.2921 & C & 2.0118 & 1.0406 & -1.8587 \\
\hline 0 & 2.1070 & -0.1362 & 7.2636 & 0 & 1.6644 & 1.9675 & -0.9439 \\
\hline C & -8.4577 & 2.3011 & 4.4734 & $\mathrm{H}$ & 2.7287 & 6.4909 & -1.9128 \\
\hline C & -7.0383 & 2.1281 & 3.9279 & $\mathrm{H}$ & 1.9069 & 7.3713 & -3.2088 \\
\hline C & -6.9869 & 1.6472 & 2.4684 & $\mathrm{H}$ & -1.6822 & 5.8470 & -0.3873 \\
\hline 0 & -7.8024 & 2.1494 & 1.6536 & $\mathrm{H}$ & -2.2813 & 7.5055 & -0.4943 \\
\hline 0 & -6.0793 & 0.8016 & 2.1757 & $\mathrm{H}$ & -3.3084 & 2.8637 & -3.4650 \\
\hline $\mathrm{H}$ & -8.4740 & 2.9036 & 5.3873 & $\mathrm{H}$ & -3.5875 & 3.3130 & -5.1356 \\
\hline $\mathrm{H}$ & -9.0908 & 2.7749 & 3.7244 & $\mathrm{H}$ & -1.0045 & 3.8696 & -3.5981 \\
\hline $\mathrm{H}$ & -6.4433 & 1.4513 & 4.5464 & $\mathrm{H}$ & -0.0366 & 4.3648 & -5.8418 \\
\hline $\mathrm{H}$ & -6.5176 & 3.0972 & 3.9396 & $\mathrm{H}$ & -1.6568 & 4.2800 & -6.5542 \\
\hline C & -0.9415 & 1.3766 & 5.6642 & $\mathrm{H}$ & -1.2673 & 5.5372 & -5.3581 \\
\hline C & -0.8939 & 1.0777 & 4.1569 & $\mathrm{H}$ & -1.6256 & 1.7195 & -5.6865 \\
\hline C & 0.3661 & 1.5466 & 3.4997 & $\mathrm{H}$ & -1.3876 & 1.4483 & -3.9536 \\
\hline C & 0.6111 & 2.4337 & 2.4689 & $\mathrm{H}$ & -0.0413 & 2.0242 & -4.9502 \\
\hline $\mathrm{N}$ & 1.6144 & 1.1038 & 3.8893 & $\mathrm{H}$ & -3.3368 & 5.7566 & -4.5033 \\
\hline C & 2.5322 & 1.7099 & 3.0908 & $\mathrm{H}$ & 1.7653 & 3.6083 & -2.5314 \\
\hline $\mathrm{N}$ & 1.9661 & 2.5276 & 2.2145 & $\mathrm{H}$ & 2.3169 & 4.7714 & -4.6265 \\
\hline $\mathrm{H}$ & -0.0877 & 0.9395 & 6.1886 & $\mathrm{H}$ & 3.3630 & 3.5197 & -6.5186 \\
\hline $\mathrm{H}$ & -1.0210 & -0.0001 & 3.9996 & $\mathrm{H}$ & 3.8468 & 1.0903 & -6.2488 \\
\hline $\mathrm{H}$ & -1.7411 & 1.5570 & 3.6536 & $\mathrm{H}$ & 3.3154 & -0.0497 & -4.1541 \\
\hline $\mathrm{H}$ & -0.1070 & 3.0169 & 1.9088 & C & -5.1886 & -3.5541 & 3.2496 \\
\hline $\mathrm{H}$ & 3.5907 & 1.5179 & 3.1766 & C & -3.7888 & -2.9871 & 3.5425 \\
\hline $\mathrm{H}$ & 2.6394 & 1.4242 & 0.1497 & 0 & -3.6095 & -1.7882 & 3.7466 \\
\hline C & 5.3056 & 6.7470 & 3.6202 & C & -6.0895 & -2.5193 & 2.5489 \\
\hline C & 5.2527 & 5.3302 & 4. 1992 & C & -5.7264 & -2.2839 & 1.0964 \\
\hline C & 5.8705 & 4.2333 & 3.3116 & $\mathrm{~N}$ & -6.5334 & -2.8459 & 0.1548 \\
\hline C & 5.8857 & 2.9013 & 4.0774 & 0 & -4.7316 & -1.6239 & 0.7616 \\
\hline C & 5.1456 & 4.0992 & 1.9648 & $\mathrm{~N}$ & -2.7744 & -3.8880 & 3.6572 \\
\hline $\mathrm{H}$ & 4.7140 & 6.8302 & 2.7032 & C & -1.4369 & -3.4423 & 4.0356 \\
\hline $\mathrm{H}$ & 4.2081 & 5.0596 & 4.4061 & C & -0.6954 & -2.7988 & 2.8560 \\
\hline $\mathrm{H}$ & 5.7669 & 5.3233 & 5.1699 & 0 & -1.0666 & -2.9783 & 1.6863 \\
\hline $\mathrm{H}$ & 138 & 4.5132 & 1022 & C & -0.7328 & -4.7582 & 4.4211 \\
\hline $\mathrm{H}$ & 4.8906 & 2.6685 & 4.4704 & C & -1.3413 & -5.7572 & 3.4257 \\
\hline $\mathrm{H}$ & 6.1871 & 2.0637 & 3.4425 & C & -2.8087 & -5.3116 & 3.3017 \\
\hline $\mathrm{H}$ & 6.5671 & 2.9468 & 4.9349 & $\mathrm{~N}$ & 0.4102 & -2.1286 & 3.2059 \\
\hline $\mathrm{H}$ & 4.0863 & 3.8515 & 2.0920 & C & 1.3921 & -1.7145 & 2.2354 \\
\hline $\mathrm{H}$ & 5.6057 & 3.3104 & 1.3627 & C & 2.7837 & -2.2318 & 2.6061 \\
\hline
\end{tabular}

$\begin{array}{llll}0 & 2.9758 & -3.3497 & 3.0770\end{array}$

$\begin{array}{llll}\mathrm{N} & 3.7535 & -1.3377 & 2.3023 \\ \mathrm{C} & 5.1770 & -1.5790 & 2.4276\end{array}$

$\begin{array}{llll}\text { C } & 5.7574 & -1.1942 & 3.8012\end{array}$

$\begin{array}{llll}0 & 6.9800 & -1.1544 & 3.9569\end{array}$

$\begin{array}{llll}\text { C } & 5.9435 & -0.8500 & 1.3196\end{array}$

$\begin{array}{llll}0 & 5.7453 & 0.5651 & 1.3675\end{array}$

$\begin{array}{llll}\mathrm{N} & 4.8762 & -0.9297 & 4.7847\end{array}$

$\begin{array}{llll}\text { C } & 5.3554 & -0.6482 & 6.1234\end{array}$

$\begin{array}{llll}H & -7.1308 & -2.8462 & 2.6117\end{array}$

$\begin{array}{llll}H & -5.9784 & -1.5470 & 3.0373\end{array}$

$\begin{array}{llll}H & -5.1384 & -4.4801 & 2.6706\end{array}$

H $\quad-0.9890 \quad-5.0230 \quad 5.4520$

$\begin{array}{llll}\mathrm{H} & 0.3527 & -4.6772 & 4.3419\end{array}$

$\begin{array}{llll}\text { H } & -1.2509 & -6.7982 & 3.7456\end{array}$

$\begin{array}{llll}\mathrm{H} & -0.8468 & -5.6506 & 2.4565\end{array}$

$\begin{array}{llll}H & -3.4584 & -5.8684 & 3.9900\end{array}$

H $\quad-3.1869-5.4576 \quad 2.2846$

$\begin{array}{llll}\mathrm{H} & -1.4983 & -2.7299 & 4.8613\end{array}$

$\begin{array}{llll}\mathrm{H} & 1.4086 & -0.6287 & 2.1313 \\ \mathrm{H} & 1.1195 & -2.1503 & 1.2740\end{array}$

$\begin{array}{llll}\mathrm{H} & 1.1195 & -2.1503 & 1.2740\end{array}$

$\begin{array}{llll}\mathrm{H} & 7.0037 & -1.0290 & 1.4689\end{array}$

$\begin{array}{llll}\text { H } & 5.6518 & -1.2444 & 0.3411\end{array}$

$\begin{array}{llll}\mathrm{H} & 4.9187 & 0.7783 & 0.8883\end{array}$

$\begin{array}{llll}\mathrm{H} & 5.3508 & -2.6558 & 2.3166\end{array}$

$\begin{array}{llll}\mathrm{H} & 6.1423 & 0.1090 & 6.0809\end{array}$

$\begin{array}{llll}\mathrm{H} & 2.0741 & 8.1214 & -1.6034\end{array}$

$\begin{array}{llll}H & -4.6707 & 4.9187 & -3.7036\end{array}$

$\begin{array}{llll}\mathrm{H} & -3.0490 & 4.7300 & -1.7332\end{array}$

$\begin{array}{lrrr}\mathrm{H} & -0.1945 & 8.2646 & -1.1192\end{array}$

$\begin{array}{llll}H & 5.7788 & -1.5438 & 6.5955\end{array}$

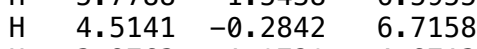

$\begin{array}{llll}\mathrm{H} & 3.8763 & -1.0720 & 4.6713\end{array}$

$\begin{array}{llll}\mathrm{H} & 0.7140 & -1.9775 & 4.1841\end{array}$

$\begin{array}{lrrr}\text { H } & -7.4367 & -3.2529 & 0.4028\end{array}$

$\begin{array}{llll}\mathrm{H} & -6.4043 & -2.5158 & -0.7966\end{array}$

$\begin{array}{llll}\mathrm{H} & 3.4699 & -0.4742 & 1.8522\end{array}$

$\begin{array}{llll}\mathrm{H} & -5.6353 & -3.8137 & 4.2170\end{array}$

$\begin{array}{lll}\text { C }-10.9767 & -5.2975 & 1.4471\end{array}$

$\begin{array}{lll}\text { C }-10.0647 & -4.4765 & 0.5580\end{array}$

$\begin{array}{llll}0 & -8.9068 & -4.2201 & 0.9015\end{array}$

$\begin{array}{lll}\text { C }-11.3693 & -4.4612 & 2.6706\end{array}$

$\begin{array}{lll}\mathrm{N}-10.6126 & -3.9952 & -0.5800\end{array}$

$\begin{array}{llll}\text { C } & -9.8884 & -3.0983 & -1.4636\end{array}$

$\begin{array}{llll}\text { C } & -9.0341 & 6.6140 & 4.0819\end{array}$

$\begin{array}{llll}\text { C } & -8.8431 & 5.4504 & 3.1190\end{array}$

C $-8.5883 \quad 5.8795 \quad 1.6743$

$\begin{array}{llll}\mathrm{N} & -8.2272 & 4.8540 & 0.8590\end{array}$

$\begin{array}{llll}0 & -8.7106 & 7.0408 & 1.2933\end{array}$

$\begin{array}{lll}\mathrm{H}-10.4727 & -4.1265 & 3.1975\end{array}$

$\begin{array}{lll}\mathrm{H}-11.9827 & -5.0440 & 3.3634\end{array}$

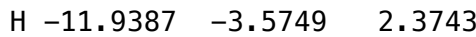

$\begin{array}{lll}H-11.8664 & -5.6353 & 0.9038\end{array}$

$\begin{array}{llll}\text { H } & -9.5772 & -2.1919 & -0.9351\end{array}$

$\begin{array}{llll}\mathrm{H} & -8.0158 & 4.8108 & 3.4394\end{array}$

$\begin{array}{llll}H & -9.7300 & 4.8052 & 3.1184\end{array}$

$\begin{array}{llll}H & -8.1236 & 3.8906 & 1.1923\end{array}$

$\begin{array}{llll}H & -8.0622 & 5.0676 & -0.1126\end{array}$

$\begin{array}{llll}\mathrm{H} & -9.8481 & 7.2626 & 3.7486\end{array}$

$\begin{array}{llll}\mathrm{H} & -9.2614 & 6.2493 & 5.0884\end{array}$

$\begin{array}{llll}\mathrm{H} & -8.1328 & 7.2315 & 4.1344\end{array}$

$\begin{array}{lll}\mathrm{H}-10.4194 & -6.1850 & 1.7598\end{array}$

H $-10.5357-2.8179-2.2949$

H $\quad-8.9925-3.5854-1.8625$

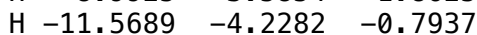

$\begin{array}{lrrr}\text { C } & 6.9518 & 0.3961 & -3.2166\end{array}$

$\begin{array}{llll}\text { C } & 6.2913 & -0.8299 & -3.1439\end{array}$

C $5.0850-0.9238-2.4471$

$\begin{array}{llll}\text { C } \quad 4.5356 & 0.1958 & -1.8168\end{array}$

$\begin{array}{llll}\text { C } & 5.2259 & 1.4131 & -1.8611\end{array}$

$\begin{array}{llll}\text { C } & 6.4165 & 1.5156 & -2.5728\end{array}$

$\begin{array}{llll}\text { H } & 7.8845 & 0.4799 & -3.7666\end{array}$

$\begin{array}{rrrr}\mathrm{H} & 7.8845 & 0.4799 & -3.7666 \\ \mathrm{H} & 6.6920 & -1.7149 & -3.6251\end{array}$

H $\quad 4.5649-1.8760 \quad-2.4046$

$\begin{array}{llll}\mathrm{H} & 4.8011 & 2.2815 & -1.3682\end{array}$

$\begin{array}{llll}\mathrm{H} & 6.9205 & 2.4748 & -2.6366\end{array}$

$\begin{array}{llll}\text { C } & 3.1916 & 0.1219 & -1.1450\end{array}$

$\begin{array}{llll}0 & 3.2525 & 0.6081 & 0.1777\end{array}$

H $\quad 2.8547-0.9188-1.1390$ 


\section{Int2(BA)-S}

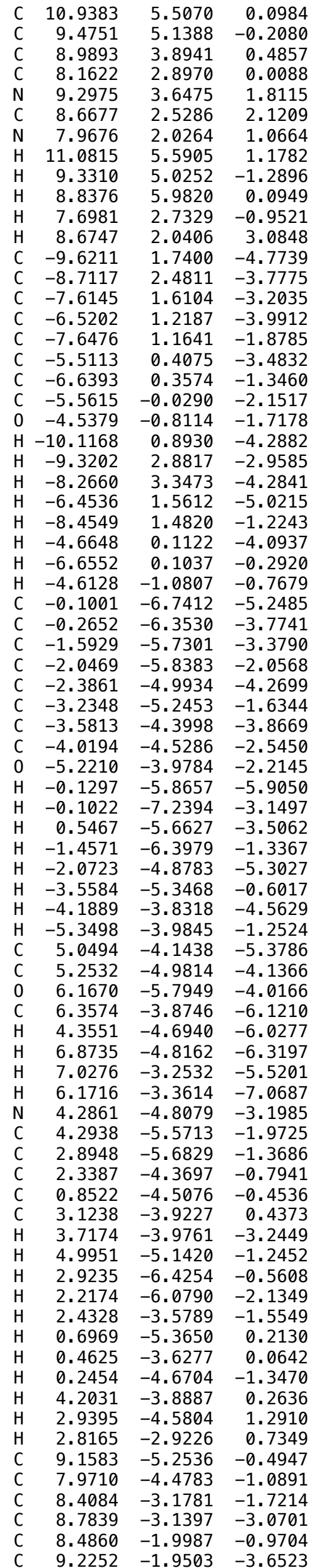

$\begin{array}{llll}\text { C } & 8.9197 & -0.8065 & -1.5481\end{array}$

$8.2924-0.7807-2.8929$
$9.6683-4.6599$

$\begin{array}{lll}9.6683 & -4.6599 & 0.2705\end{array}$

$\begin{array}{lll}7.2414 & -4.2715 & -0.2959\end{array}$

$\begin{array}{lll}7.4686 & -5.0989 & -1.8390\end{array}$

$\begin{array}{lll}8.7077 & -4.0465 & -3.6645\end{array}$

$\begin{array}{lll}8.1860 & -2.0120 & 0.0735\end{array}$

$\begin{array}{lll}9.5105 & -1.9359 & -4.7009\end{array}$

$\begin{array}{lll}8.9567 & 0.1012 & -0.9530\end{array}$

$\begin{array}{llll}9.6275 & 0.1474 & -3.3465\end{array}$

$\begin{array}{lll}-2.8571 & 0.9214 & 0.5076\end{array}$

$\begin{array}{lll}-1.6591 & 1.3747 & 0.0217\end{array}$

$\begin{array}{llll}C & -1.6670 & 2.6999 & -0.5581\end{array}$

$\begin{array}{llll}\mathrm{N} & -0.5620 & 3.3380 & -0.8630\end{array}$

$\begin{array}{llll}\mathrm{N} & -2.8650 & 3.3257 & -0.8023\end{array}$

C $\quad-3.9829 \quad 2.7961 \quad-0.3521$

$\begin{array}{llll}\mathrm{N} & -4.0063 & 1.6403 & 0.3579\end{array}$

$\begin{array}{lll}1.9691 & -0.4344 & -2.7931\end{array}$

$\begin{array}{lll}1.4467 & 0.5899 & -1.5144\end{array}$

$\begin{array}{llll}C & -5.2752 & 3.4721 & -0.6635\end{array}$

$\begin{array}{llll}N & 0.2986 & 0.1076 & -0.9956\end{array}$

C $\quad-0.2148 \quad-1.0470 \quad-1.6264$

$\begin{array}{llll}\text { C } & -1.5141 & -1.6554 & -1.2191\end{array}$

$\begin{array}{lll}0.5868 & -1.4692 & -2.6439\end{array}$

$0.3888-2.5993-3.6030$

$\begin{array}{lll}-0.3772 & 0.6161 & 0.2552\end{array}$

$\begin{array}{llll}\mathrm{H} & 0.4551 & 3.0004 & -0.5325\end{array}$

$\begin{array}{llll}H & -0.6456 & 4.3260 & -1.0912\end{array}$

$\begin{array}{llll}H & -0.5789 & -0.2699 & 0.8564\end{array}$

$\begin{array}{rrr}0.3724 & 1.2350 & 0.7445\end{array}$

$\begin{array}{llll}-6.0141 & 3.2910 & 0.1227\end{array}$

$\begin{array}{llll}\mathrm{H} & -5.1026 & 4.5396 & -0.8056\end{array}$

$\begin{array}{llll}\text { H } & -5.6746 & 3.0544 & -1.5945\end{array}$

$\begin{array}{llll}H & -2.9662 & -0.0191 & 1.0397\end{array}$

$\begin{array}{llll}\mathrm{H} & -1.5553 & -1.8710 & -0.1497\end{array}$

H $\quad-1.6506 \quad-2.6042 \quad-1.7381$

H $\quad-2.3528 \quad-1.0124-1.4920$

$\begin{array}{lll}1.2384 & -3.2901 & -3.5904\end{array}$

$\begin{array}{llll}0.2753 & -2.2268 & -4.6266\end{array}$

$\begin{array}{lll}1.3124 & -3.1356 & 7.4386\end{array}$

$\begin{array}{lll}0.6378 & -1.7709 & 7.5850\end{array}$

$\begin{array}{lll}1.3122 & -0.6995 & 6.7211\end{array}$

$\begin{array}{lll}1.7012 & -1.0665 & 5.5698\end{array}$

$\begin{array}{lll}1.4137 & 0.4682 & 7.1899\end{array}$

$\begin{array}{lll}-8.8742 & 2.1160 & 3.7652\end{array}$

$\begin{array}{lll}-7.8382 & 1.0927 & 3.2945\end{array}$

$\begin{array}{llll}C & -7.0123 & 1.5350 & 2.0782\end{array}$

$\begin{array}{llll}0 & -7.3278 & 2.5993 & 1.4940\end{array}$

$\begin{array}{llll}0 & -6.0486 & 0.7632 & 1.7518\end{array}$

$\begin{array}{llll}\mathrm{H} & -9.4401 & 1.7312 & 4.6211\end{array}$

$\begin{array}{llll}H & -8.3937 & 3.0491 & 4.0680\end{array}$

$\begin{array}{llll}\mathrm{H} & -8.3210 & 0.1423 & 3.0299\end{array}$

$\begin{array}{llll}H & -7.1295 & 0.8457 & 4.0932\end{array}$

$\begin{array}{llll}C & -1.4254 & 1.5818 & 5.4963\end{array}$

$\begin{array}{llll}-0.7626 & 2.7916 & 4.8139\end{array}$

$\begin{array}{lll}0.5531 & 2.4624 & 4.1829\end{array}$

$\begin{array}{lll}1.0209 & 2.6147 & 2.8957\end{array}$

$\begin{array}{llll}\mathrm{N} & 1.5775 & 1.8486 & 4.8764\end{array}$

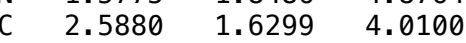

$\begin{array}{llll}\mathrm{N} & 2.2963 & 2.0847 & 2.7960\end{array}$

$\begin{array}{lrll}\mathrm{H} & -1.5705 & 0.7618 & 4.7856\end{array}$

$\begin{array}{llll}\text { H } & -1.4185 & 3.1917 & 4.0330\end{array}$

$\mathrm{H} \quad-0.6355 \quad 3.5953 \quad 5.5523$

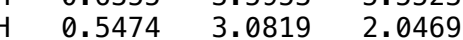

$\begin{array}{llll}H & 3.4933 & 1.1132 & 4.2831\end{array}$

$\begin{array}{lll}3.4933 & 1.1132 & 4.2831 \\ 2.9441 & 1.4114 & 1.4622\end{array}$

$\begin{array}{lll}4.7579 & 7.0115 & 3.4155\end{array}$

$\begin{array}{lll}4.8265 & 5.5845 & 3.9672\end{array}$

$\begin{array}{lll}5.6050 & 4.5723 & 3.1044\end{array}$

$\begin{array}{lll}5.7518 & 3.2561 & 3.8819\end{array}$

$\begin{array}{lll}4.9386 & 4.3303 & 1.7415\end{array}$

$\begin{array}{lll}4.2190 & 7.0515 & 2.4635\end{array}$

$3.8078 \quad 5.1998 \quad 4.1133$

$5.2866 \quad 5.6123 \quad 4.9642$

$\begin{array}{lll}6.6121 & 4.9737 & 2.9195\end{array}$

$\begin{array}{lll}4.7815 & 2.9404 & 4.2729\end{array}$

$\begin{array}{lll}6.1307 & 2.4448 & 3.2559\end{array}$

$\begin{array}{lll}6.4320 & 3.3747 & 4.7327\end{array}$

$\begin{array}{lll}3.9466 & 3.8815 & 1.8605\end{array}$

$\begin{array}{lll}5.5542 & 3.6530 & 1.1396\end{array}$

$\begin{array}{lll}4.8294 & 5.2614 & 1.1738\end{array}$

\begin{tabular}{|c|c|c|c|}
\hline & -0.5068 & -3.1687 & -3.3569 \\
\hline & -9.5763 & 526 & 9639 \\
\hline & 220 & 7340 & 2673 \\
\hline & 11.2081 & 584 & -0 \\
\hline & -10.3926 & 1049 & -5.1761 \\
\hline & -9.0396 & 1.3456 & -5.613 \\
\hline & -0.8051 & 33 & 6. 317 \\
\hline & -2.4047 & & \\
\hline & 5.7625 & 7.4171 & 3.245 \\
\hline & & & 11 \\
\hline & -0.4101 & -1 & \\
\hline & 0.6 & -1 . & \\
\hline & 1.4074 & -3 & \\
\hline & & & \\
\hline & & & \\
\hline & 8.8 & -6 . & -0 . \\
\hline & 9.8 & -5 & -1 \\
\hline & & & \\
\hline & 0.8 & -7 . & -5 . \\
\hline & -0.8 & -7 & -5 . \\
\hline & 4. & -3 & -5 . \\
\hline & 1. & & \\
\hline & -4.5 & & \\
\hline & 1.7344 & 6.8 & -2 . \\
\hline & 0.3 & & -1.8906 \\
\hline & 0.2 & & \\
\hline & -0.6 & & -2 \\
\hline & -2.6 & & -2 . \\
\hline & -2. & & -3 \\
\hline & -2 & & -4 \\
\hline & -3.6 & & -3 \\
\hline & -3.5 & & -4 \\
\hline & -3 & & \\
\hline & -1. & & -4 \\
\hline & -1.2 & & -4 \\
\hline & -0.8 & & -5 . \\
\hline & & & \\
\hline & 3.2 & & -1 \\
\hline & 4.13 & & -2 \\
\hline & & & -4 \\
\hline & & & \\
\hline & 2.2 & & -3 \\
\hline & 2.2 & & -1 \\
\hline & 1.6 & & \\
\hline & & & \\
\hline & 2. & & -1 \\
\hline & -2.6 & & -1 . \\
\hline & -2 & & \\
\hline & -3 & & -3 \\
\hline & -3.2 & & -5 \\
\hline & -1.1 & & -3 \\
\hline & & & \\
\hline & -1 & & \\
\hline & -1.0 & & -5 \\
\hline & -1.5 & & -5 . \\
\hline & -1 & & \\
\hline & -0. & & \\
\hline & -3.2 & 4. & -5 \\
\hline & & & -0 \\
\hline & & & \\
\hline & 4.2 & & \\
\hline & 2. & & -5 \\
\hline & 1.8 & & \\
\hline & & & \\
\hline & -3. & & \\
\hline & -3.9 & -1 & 4 \\
\hline & -6 & & \\
\hline & & & \\
\hline & & & \\
\hline & -4 & & \\
\hline & & & \\
\hline & -1 . & & \\
\hline & -0.8 & & \\
\hline & & & \\
\hline & -0. & -4 & 587 \\
\hline & -1.2 & & \\
\hline & -2.77 & & \\
\hline & & & \\
\hline & 1.2 & 1. & \\
\hline & 2. & & \\
\hline & .5 & & \\
\hline
\end{tabular}




\begin{tabular}{|c|c|c|c|}
\hline N & 3.6626 & -1.4351 & 2.7493 \\
\hline C & 5.0189 & -1.8179 & 3.0861 \\
\hline & 5.4947 & -1.2474 & 4.4400 \\
\hline c & 6.6948 & -1.2339 & 4.7136 \\
\hline c & 6.0039 & -1.3644 & 1.9995 \\
\hline C & 5.9675 & 0.0435 & 1.780 \\
\hline & 4.5349 & -0.7594 & 5.2499 \\
\hline & 4.8923 & -0.1537 & 6.513 \\
\hline & -7.2185 & -2.9201 & 2.472 \\
\hline H & -6.0674 & -1.6698 & 3.011 \\
\hline H & -5.2344 & -4.5955 & 2.6344 \\
\hline H & -1.0575 & -5.1983 & 5.1095 \\
\hline & 0.2913 & -4.5589 & 38 \\
\hline H & -1.1625 & -6.6327 & \\
\hline $\mathrm{H}$ & -0.7543 & -5.2583 & 2.0637 \\
\hline ト & -3.4352 & -5.9100 & 3.2924 \\
\hline ト & -3.0649 & -5.0595 & 1.773 \\
\hline $\mathrm{H}$ & -1.7 & -2 . & \\
\hline $\mathrm{H}$ & 1.4117 & -0.3167 & 2. \\
\hline H & 1.0339 & -1.6420 & 1.5922 \\
\hline H & 7.0080 & -1.5955 & 3571 \\
\hline $\mathrm{H}$ & 5.8 & -1 . & \\
\hline $\mathrm{H}$ & 5.0908 & 35 & 1 \\
\hline $\mathrm{H}$ & 5.0467 & -2.9105 & 3.1604 \\
\hline $\mathrm{H}$ & 5.6455 & 0.6234 & 6.3551 \\
\hline $\mathrm{H}$ & 1.8690 & 86 & -3 \\
\hline $\mathrm{H}$ & -4.6538 & 4. & -4 \\
\hline $\mathrm{H}$ & -3 & 4. & 3207 \\
\hline $\mathrm{H}$ & -0.4739 & 7.8060 & -3.4053 \\
\hline $\mathrm{H}$ & 5.3143 & -0.8 & 7. \\
\hline $\mathrm{H}$ & 3. & 0. & \\
\hline $\mathrm{H}$ & 3.5415 & -0 . & 60 \\
\hline $\mathrm{H}$ & 0.3977 & -1.8036 & 4.4508 \\
\hline $\mathrm{H}$ & -7.5292 & -2.9840 & 0. \\
\hline $\mathrm{H}$ & -6.4337 & -2.2 & -0 \\
\hline $\mathrm{H}$ & 3.5291 & -0. & 208 \\
\hline $\mathrm{H}$ & -5.8698 & -3 & 4.1500 \\
\hline$C$ & -10.4646 & -5.6534 & 1.4434 \\
\hline $\mathrm{C}$ & -9.8114 & -4.6754 & 0.4907 \\
\hline 0 & -9.0528 & -3 & 46 \\
\hline$C$ & -11.4316 & -4.8761 & 2.3427 \\
\hline $\mathrm{N}$ & -10.1757 & -4.7614 & -0.8075 \\
\hline $\mathrm{C}$ & -9.7294 & -3.7896 & -1.7886 \\
\hline C & -9.5686 & 85 & 367 \\
\hline C & -9.4312 & 5. & 9933 \\
\hline$C$ & -9.3205 & 5.4583 & 0.6059 \\
\hline $\mathrm{N}$ & -8.8120 & 4.4326 & -0.1392 \\
\hline 0 & -9.6966 & 12 & 02 \\
\hline $\mathrm{H}$ & -10.8963 & -4 & 593 \\
\hline $\mathrm{H}$ & -11.8869 & -5.5321 & 3.0899 \\
\hline $\mathrm{H}$ & -12.2347 & -4.4228 & 1.7532 \\
\hline $\mathrm{H}$ & -10.9854 & -6 . & 54 \\
\hline $\mathrm{H}$ & -9.7460 & -2 & -1.3445 \\
\hline $\mathrm{H}$ & -8.5724 & 77 & 2.3750 \\
\hline $\mathrm{H}$ & -10.3089 & 4.4814 & 2.2113 \\
\hline $\mathrm{H}$ & -8.3185 & 3.6 & 0. \\
\hline $\mathrm{H}$ & -8.6190 & 4. & -1 \\
\hline $\mathrm{H}$ & -10.4022 & 6. & 5576 \\
\hline $\mathrm{H}$ & -9.7349 & 6.0628 & 4.0272 \\
\hline $\mathrm{H}$ & -8.6652 & 6. & 487 \\
\hline $\mathrm{H}$ & -9.6749 & -6 & 52 \\
\hline $\mathrm{H}$ & -10.4010 & -3.8104 & -2.6483 \\
\hline $\mathrm{H}$ & -8.7086 & -3.9940 & -2.1328 \\
\hline $\mathrm{H}$ & -10.7737 & -5.5184 & -1.0979 \\
\hline C & 5.8015 & -1.4083 & -2 . \\
\hline C & 5.1514 & -1.8 & -1.8629 \\
\hline C & 4.4804 & -1.0152 & -1.0140 \\
\hline C & 4.4760 & 0.3600 & -1.2661 \\
\hline C & 5.1783 & 0.8453 & -2.379 \\
\hline C & 5.8213 & -0.0363 & -3 \\
\hline $\mathrm{H}$ & 6.3458 & -2.0854 & -3.6422 \\
\hline $\mathrm{H}$ & 5.1838 & -2.9479 & -1.6325 \\
\hline $\mathrm{H}$ & 3.9451 & -1.3856 & -0.1512 \\
\hline 11 & 5.2120 & 1.9112 & -2.5725 \\
\hline $\mathrm{H}$ & 6.3632 & 0.3501 & -4.1034 \\
\hline$C$ & 687 & 1.2905 & -0.4040 \\
\hline 0 & & 0.7173 & 0.848 \\
\hline $\mathrm{H}$ & 7.3256 & 1.2314 & 1.118 \\
\hline & & & \\
\hline
\end{tabular}

\section{TS3(BA)-R}

$\begin{array}{rrrr}\mathrm{C} & 11.2048 & 5.2709 & -0.4190 \\ \mathrm{C} & 11.1235 & 3.7383 & -0.5323 \\ \mathrm{C} & 10.0604 & 3.1212 & 0.3305 \\ \mathrm{C} & 8.9444 & 2.4084 & -0.0533 \\ \mathrm{~N} & 10.1039 & 3.2096 & 1.7108 \\ \mathrm{C} & 9.0339 & 2.5580 & 2.1378 \\ \mathrm{~N} & 8.2968 & 2.0530 & 1.1103\end{array}$

$\begin{array}{llll}\mathrm{H} & 10.2720 & 5.7354 & -0.7559\end{array}$

$\begin{array}{llll}H & 12.0977 & 3.3144 & -0.2547\end{array}$

$\begin{array}{llll}\text { H } & 10.9447 & 3.4506 & -1.5757\end{array}$

$\begin{array}{llll}\mathrm{H} & 8.5685 & 2.1129 & -1.0179\end{array}$

$\begin{array}{llll}\mathrm{H} & 8.7484 & 2.4148 & 3.1699\end{array}$

$\begin{array}{llll}\mathrm{H} & 7.4419 & 1.4878 & 1.1878\end{array}$

$\begin{array}{llll}\text { C } & -9.7938 & 2.6066 & -3.9664\end{array}$

$\begin{array}{llll}\text { C } & -8.5378 & 3.1962 & -3.3039\end{array}$

$\begin{array}{llll}\text { C } & -7.5923 & 2.1095 & -2.8498\end{array}$

$\begin{array}{llll}\text { C } & -6.8222 & 1.4061 & -3.7864\end{array}$

$\begin{array}{llll}C & -7.5185 & 1.7162 & -1.5100\end{array}$

$\begin{array}{llll}\text { C } & -6.0357 & 0.3243 & -3.4064\end{array}$

$\begin{array}{llll}\text { C } & -6.7253 & 0.6419 & -1.1058\end{array}$

C $\quad-6.0008-0.0728-2.0650$

$\begin{array}{llll}0 & -5.2621 & -1.1791 & -1.7570\end{array}$

$\begin{array}{lll}\mathrm{H}-10.3271 & 1.9580 & -3.2641\end{array}$

$\begin{array}{llll}\mathrm{H} & -8.8329 & 3.8135 & -2.4474\end{array}$

$\begin{array}{llll}H & -8.0352 & 3.8639 & -4.0154\end{array}$

H $\quad-6.8458 \quad 1.7063 \quad-4.8318$

$\begin{array}{llll}\mathrm{H} & -8.0824 & 2.2383 & -0.7431\end{array}$

H $\quad-5.4412 \quad-0.2263 \quad-4.1283$

$\begin{array}{llll}\mathrm{H} & -6.6641 & 0.3940 & -0.0497\end{array}$

$\begin{array}{llll}\text { H } & -5.0544 & -1.2127 & -0.7862\end{array}$

C $-0.7089-6.2014-5.6070$

$\begin{array}{llll}\text { C } & -0.7915 & -6.1954 & -4.0758\end{array}$

C $\quad-2.1502-5.8734-3.4746$

$\begin{array}{llll}\text { C } & -2.2540 & -5.6078 & -2.1019\end{array}$

$\begin{array}{llll}\text { C } & -3.3228 & -5.7959 & -4.2347\end{array}$

C $-3.4680-5.2691-1.5079$

C $\quad-4.5468-5.4615-3.6566$

C $-4.6293-5.1932-2.2875$

$\begin{array}{llll}0 & -5.8543 & -4.8856 & -1.7744\end{array}$

H $\quad-1.0210 \quad-5.2409-6.0294$

H $-0.4519-7.1700 \quad-3.6989$

H $\quad-0.0668 \quad-5.4683-3.6860$

H $-1.3651-5.6502-1.4801$

H $\quad-3.2898 \quad-5.9832 \quad-5.3029$

H $\quad-3.5076 \quad-5.0424-0.4453$

$\begin{array}{llll}H & -5.4487 & -5.3945 & -4.2556\end{array}$

$\begin{array}{llll}\text { H } & -5.7755 & -4.5843 & -0.8537\end{array}$

$\begin{array}{lll}4.5267 & -3.8057 & -5.9714\end{array}$

$\begin{array}{lll}4.8374 & -4.1855 & -4.5430\end{array}$

$5.9328-3.9816-4.0226$

$\begin{array}{lll}5.1647 & -2.4680 & -6.3564\end{array}$

$\begin{array}{lll}4.9223 & -4.6034 & -6.6134\end{array}$

$\begin{array}{lll}6.2422 & -2.5058 & -6.1851\end{array}$

$\begin{array}{lll}4.7664 & -1.6522 & -5.7457\end{array}$

$\begin{array}{lll}4.9812 & -2.2346 & -7.4090\end{array}$

$3.8079-4.7908-3.9033$

$\begin{array}{llll}3.9542 & -5.3980 & -2.6028\end{array}$

$\begin{array}{lll}2.6653 & -5.2987 & -1.7947\end{array}$

$2.3250 \quad-3.8707 \quad-1.3471$

$\begin{array}{lll}0.8906 & -3.8075 & -0.8234\end{array}$

$\begin{array}{lll}3.3235 & -3.3888 & -0.2915\end{array}$

$\begin{array}{lll}2.9570 & -4.9665 & -4.4164\end{array}$

$\begin{array}{lll}4.7831 & -4.8929 & -2.1046\end{array}$

$\begin{array}{lll}2.7534 & -5.9396 & -0.9085\end{array}$

$\begin{array}{lll}1.8410 & -5.7170 & -2.3912\end{array}$

$2.3958-3.2130 \quad-2.2264$

$\begin{array}{lll}0.7505 & -4.4785 & 0.0316\end{array}$

$0.6234-2.8092-0.4808$

$0.1709-4.0829-1.5990$

$\begin{array}{lll}4.3596 & -3.4478 & -0.6380\end{array}$

$\begin{array}{lll}3.2393 & -3.9788 & 0.6263\end{array}$

$\begin{array}{lll}3.1451 & -2.3485 & -0.0185\end{array}$

$8.9205-5.3587-1.4721$

$\begin{array}{lll}7.8575 & -4.3682 & -0.9664\end{array}$

$8.4723-3.2250-0.1917$

$8.9167-2.0736-0.8549$

$8.6550-3.3058 \quad 1.1950$

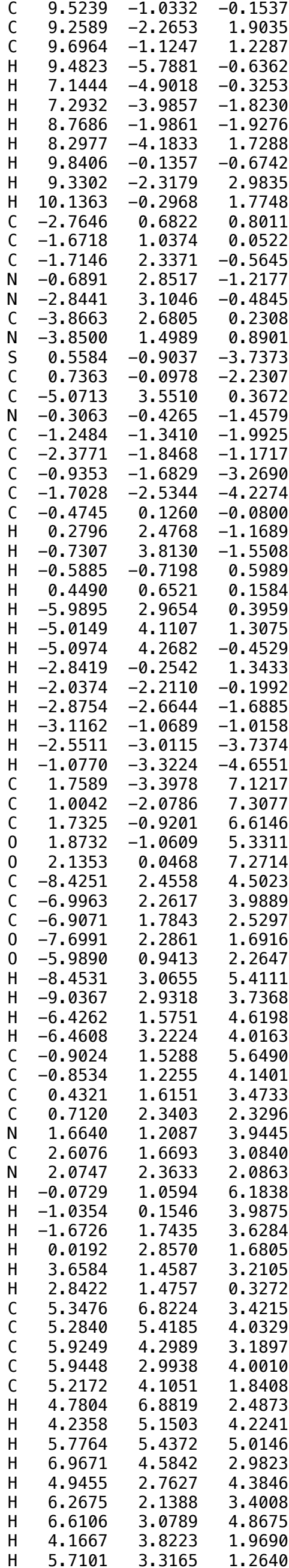




\begin{tabular}{|c|c|c|c|}
\hline $\mathrm{H}$ & 5.2497 & 5.0172 & 1.2352 \\
\hline $\mathrm{H}$ & -2.0885 & -1.9296 & -5.0557 \\
\hline 4 & -8.8852 & 1.4888 & 4.7354 \\
\hline 11 & 11.3610 & 5.5584 & 0.6243 \\
\hline $\mathrm{H}$ & 12.0277 & 5.6719 & -1.0207 \\
\hline & -10.4812 & 3.3927 & -4.2978 \\
\hline $\mathrm{H}$ & -9.5231 & 2.0000 & -4.8366 \\
\hline $\mathrm{H}$ & -0.8369 & 2.6069 & 5.8287 \\
\hline $\mathrm{H}$ & -1.8409 & 1.1644 & 6.0769 \\
\hline $\mathrm{H}$ & 6.3825 & 7.1079 & 3.1990 \\
\hline $\mathrm{H}$ & 4.9340 & 7.5702 & 4.1065 \\
\hline 1 & 0.0016 & -2.1617 & 6.8653 \\
\hline H & 0.8825 & -1.8349 & 8.3664 \\
\hline 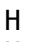 & 1.9559 & -3.5742 & 6.0605 \\
\hline $\mathrm{H}$ & 1.1987 & -4.2490 & 7.5256 \\
\hline $\mathrm{H}$ & 2.7274 & -3.3621 & 7.6319 \\
\hline $\mathrm{H}$ & 8.4614 & -6.1762 & -2.0386 \\
\hline $\mathrm{H}$ & 9.6360 & -4.8514 & -2.1271 \\
\hline $\mathrm{H}$ & 4.2510 & -6.4516 & -2.7109 \\
\hline H & 0.3173 & -6.3969 & -5.9333 \\
\hline $\mathrm{H}$ & -1.3454 & -6.9781 & -6.0420 \\
\hline $\mathrm{H}$ & 3.4430 & -3.7771 & -6.1352 \\
\hline $\mathrm{H}$ & 1.8251 & 0.4662 & 4.6640 \\
\hline $\mathrm{H}$ & -4.7253 & 1.2081 & 1.4543 \\
\hline C & 1.9027 & 7.1301 & -2.3087 \\
\hline C & 0.6122 & 6.4583 & -1.9026 \\
\hline 0 & 0.3965 & 5.2698 & -2.1555 \\
\hline $\mathrm{N}$ & -0.3029 & 7.2210 & -1.2487 \\
\hline C & -1.6569 & 6.7110 & -1.1204 \\
\hline C & -2.2520 & 6.4432 & -2.5148 \\
\hline 0 & -2.0192 & 7.2150 & -3.4427 \\
\hline $\mathrm{N}$ & -3.0308 & 5.3434 & -2.5957 \\
\hline C & -3.6075 & 4.9082 & -3.8594 \\
\hline C & -3.0989 & 3.5278 & -4.2843 \\
\hline C & -1.6002 & 3.4507 & -4.6131 \\
\hline C & -1.1949 & 1.9923 & -4.8429 \\
\hline C & -1.2265 & 4.3353 & -5.8083 \\
\hline C & 2.5165 & 1.6912 & -3.2861 \\
\hline C & 2.1931 & 3.0108 & -3.6192 \\
\hline C & 2.3724 & 3.4733 & -4.9225 \\
\hline C & 2.8714 & 2.6245 & -5.9104 \\
\hline C & 3.2003 & 1.3072 & -5.5826 \\
\hline C & 3.0314 & 0.8469 & -4.2798 \\
\hline C & 2.3079 & 1.2705 & -1.8473 \\
\hline 0 & 1.8472 & 2.1324 & -1.0456 \\
\hline $\mathrm{H}$ & 2.7372 & 6.4776 & -2.0441 \\
\hline $\mathrm{H}$ & 1.8990 & 7.2489 & -3.3964 \\
\hline $\mathrm{H}$ & -1.6593 & 5.8115 & -0.4995 \\
\hline $\mathrm{H}$ & -2.2621 & 7.4681 & -0.6154 \\
\hline $\mathrm{H}$ & -3.3383 & 2.8070 & -3.4912 \\
\hline $\mathrm{H}$ & -3.6782 & 3.2090 & -5.1624 \\
\hline $\mathrm{H}$ & -1.0366 & 3.8210 & -3.7508 \\
\hline $\mathrm{H}$ & -0.1729 & 4.1968 & -6.0684 \\
\hline $\mathrm{H}$ & -1.8251 & 4.0788 & -6.6923 \\
\hline $\mathrm{H}$ & -1.3784 & 5.3949 & -5.5832 \\
\hline $\mathrm{H}$ & -1.7348 & 1.5631 & -5.6973 \\
\hline $\mathrm{H}$ & -1.4246 & 1.3814 & -3.9639 \\
\hline $\mathrm{H}$ & -0.1240 & 1.9039 & -5.0397 \\
\hline $\mathrm{H}$ & -3.3680 & 5.6759 & -4.5958 \\
\hline $\mathrm{H}$ & 1.8014 & 3.6720 & -2.8566 \\
\hline $\mathrm{H}$ & 2.1139 & 4.5007 & -5.1603 \\
\hline $\mathrm{H}$ & 3.0089 & 2.9849 & -6.9257 \\
\hline $\mathrm{H}$ & 3.5998 & 0.6380 & -6.3390 \\
\hline $\mathrm{H}$ & 3.3181 & -0.1683 & -4.0374 \\
\hline C & -5.1903 & -3.4407 & 3. 3912 \\
\hline C & -3.7887 & -2.8513 & 3.6280 \\
\hline 0 & -3.6289 & -1.6540 & 3.8554 \\
\hline C & -6.1143 & -2.4409 & 2.6708 \\
\hline C & -5.7802 & -2.2777 & 1.2029 \\
\hline $\mathrm{N}$ & -6.5794 & -2.9174 & 0.3063 \\
\hline 0 & -4.8069 & -1.6076 & 0.8237 \\
\hline $\mathrm{N}$ & -2.7493 & -3.7292 & 3.6689 \\
\hline C & -1.4123 & -3.2707 & 4.0329 \\
\hline C & -0.6930 & -2.5960 & 2.8573 \\
\hline 0 & -1.0893 & -2.7322 & 1.6900 \\
\hline ( & -0.6812 & -4.5839 & 4.3788 \\
\hline $\mathrm{C}$ & -1.2922 & -5.5690 & 3.3712 \\
\hline $\mathrm{C}$ & -2.7693 & -5.1473 & 3.2902 \\
\hline 1 & 0.4318 & -1.9549 & 3.2031 \\
\hline & 1.4255 & -1.5794 & 2.2286 \\
\hline & & & \\
\hline
\end{tabular}

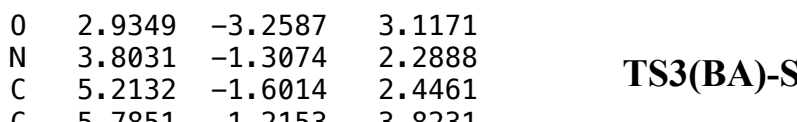

C $\quad 5.7851-1.2153 \quad 3.8231$

$\begin{array}{llll}0 & 7.0025 & -1.2620 & 4.0092\end{array}$

$\begin{array}{llll}\text { C } & 6.0256 & -0.9037 & 1.3498\end{array}$

$\begin{array}{llll}0 & 5.9008 & 0.5194 & 1.4129\end{array}$

N $\quad 4.9074 \quad-0.8297 \quad 4.7714$

$\begin{array}{llll}\text { C } & 5.3902 & -0.5106 & 6.1015\end{array}$

H $\quad-7.1526 \quad-2.7688 \quad 2.7725$

H $\quad-5.9941-1.4490 \quad 3.1161$

H $\quad-5.1524 \quad-4.3916 \quad 2.8536$

$\mathrm{H} \quad-0.9168-4.8755 \quad 5.4074$

$\begin{array}{llll}H & 0.4014 & -4.4846 & 4.2819\end{array}$

H $\quad-1.1765-6.6159 \quad 3.6623$

$\mathrm{H} \quad-0.8201 \quad-5.4294 \quad 2.3949$

H $\quad-3.3908 \quad-5.7269 \quad 3.9857$

$\mathrm{H} \quad-3.1716-5.2832 \quad 2.2809$

H $\quad-1.4702 \quad-2.5755 \quad 4.8737$

H $\quad 1.4772 \quad-0.4963 \quad 2.1074$

H $1.1415 \quad-2.0148 \quad 1.2693$

H $7.0728 \quad-1.1440 \quad 1.5004$

H $\quad 5.7175 \quad-1.2689 \quad 0.3646$

$\begin{array}{llll}\text { H } & 5.0939 & 0.7778 & 0.9224\end{array}$

H $\quad 5.3526 \quad-2.6840 \quad 2.3473$

$\begin{array}{llll}H & 6.1824 & 0.2399 & 6.0369\end{array}$

H $2.0439 \quad 8.1085-1.8438$

$\mathrm{H} \quad-4.7003 \quad 4.8718 \quad-3.7609$

H $\quad-3.0377 \quad 4.6805-1.8193$

$\mathrm{H} \quad-0.1934 \quad 8.2221 \quad-1.3071$

$\begin{array}{llll}H & 5.8081 & -1.3939 & 6.6000\end{array}$

$\begin{array}{llll}\mathrm{H} & 4.5520 & -0.1224 & 6.6827\end{array}$

$\begin{array}{llll}H & 3.9048 & -0.9532 & 4.6619\end{array}$

H $\quad 0.7463 \quad-1.8330 \quad 4.1813$

$\mathrm{H} \quad-7.4752-3.3215 \quad 0.5858$

H $\quad-6.4696-2.6365-0.6646$

$\mathrm{H} \quad 3.5633 \quad-0.4506 \quad 1.8013$

H $\quad-5.6091 \quad-3.6552 \quad 4.3818$

C $-11.0884 \quad-5.1949 \quad 1.6532$

$\begin{array}{lll}\text { C }-10.1478 & -4.4327 & 0.7428\end{array}$

$\begin{array}{llll}0 & -8.9747 & -4.2316 & 1.0722\end{array}$

$\begin{array}{lll}\mathrm{C}-11.3795 & -4.3357 & 2.8885\end{array}$

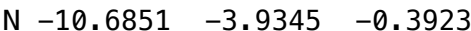

C $\quad-9.9299-3.0787 \quad-1.2902$

$\begin{array}{llll}\text { C } & -8.9879 & 6.7604 & 4.0121\end{array}$

$\begin{array}{llll}C & -8.7857 \quad 5.5860 & 3.0653\end{array}$

C $\quad-8.5129 \quad 5.9990 \quad 1.6194$

N $\quad-8.1321 \quad 4.9654 \quad 0.8236$

$\begin{array}{llll}0 & -8.6408 & 7.1537 & 1.2206\end{array}$

H $-10.4439-4.0705 \quad 3.3860$

$\mathrm{H}-12.0128-4.8723 \quad 3.6004$

H $-11.8901-3.4084 \quad 2.6105$

H $-12.0164-5.4661 \quad 1.1375$

H $\quad-9.5606 \quad-2.1908-0.7679$

$\begin{array}{llll}H & -7.9638 & 4.9481 & 3.4029\end{array}$

H $\quad-9.6738 \quad 4.9424 \quad 3.0598$

H $\quad-8.0266 \quad 4.0090 \quad 1.1749$

H $\quad-7.9624 \quad 5.1648 \quad-0.1501$

$\mathrm{H} \quad-9.7966 \quad 7.4061 \quad 3.6606$

$\mathrm{H} \quad-9.2291 \quad 6.4076 \quad 5.0196$

$\mathrm{H} \quad-8.0867 \quad 7.3777 \quad 4.0699$

$\begin{array}{lll}\mathrm{H}-10.5863 & -6.1211 & 1.9473\end{array}$

H $-10.5763 \quad-2.7632 \quad-2.1094$

H $\quad-9.0688-3.6131-1.7048$

$\begin{array}{llll}H & -11.6539 & -4.1232 & -0.5930\end{array}$

$\begin{array}{lrrr}\text { C } & 7.1371 & 0.3170 & -3.2287\end{array}$

$\begin{array}{llll}\text { C } & 6.4163 & -0.8707 & -3.1075\end{array}$

$\begin{array}{llll}\text { C } & 5.2088 & -0.8716 & -2.4083\end{array}$

C $\quad 4.7129 \quad 0.3019-1.8308$

C $5.4556 \quad 1.4823-1.9332$

C $\quad 6.6547 \quad 1.4908 \quad-2.6414$

$\mathrm{H} \quad 8.0753 \quad 0.3299-3.7754$

H $\quad 6.7680 \quad-1.7961-3.5530$

H $\quad 4.6414-1.7939-2.3252$

H $5.0748 \quad 2.3935-1.4816$

$\mathrm{H} \quad 7.2085 \quad 2.4187 \quad-2.7461$

$\begin{array}{llll}\text { C } & 3.3585 & 0.2980 & -1.1646\end{array}$

$\begin{array}{lllr}\text { C } & 3.4559 & 0.7018 & 0.1975\end{array}$

$\mathrm{H} \quad 2.9701-0.7210 \quad-1.1978$

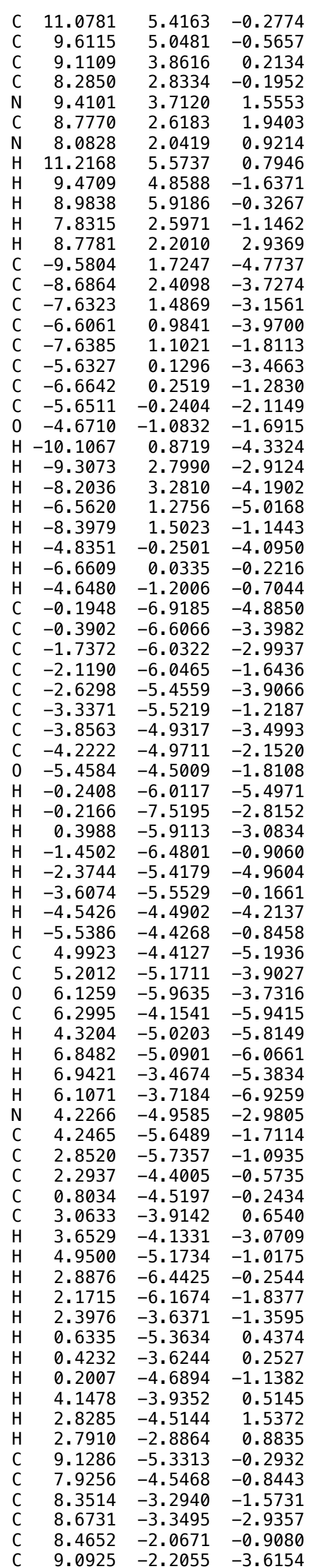




\begin{tabular}{|c|c|c|c|c|c|c|c|}
\hline C & 8.8788 & -0.9196 & -1.5841 & $\mathrm{H}$ & -0.6781 & -3.4663 & -3.2845 \\
\hline C & 9.1928 & -0.9865 & -2.9419 & $\mathrm{H}$ & -9.4357 & 2.7618 & 2.9109 \\
\hline $\mathrm{H}$ & 9.7049 & -4.7186 & 0.4084 & $\mathrm{H}$ & 11.7518 & 4.6092 & -0.5832 \\
\hline $\mathrm{H}$ & 7.2563 & -4.2797 & -0.0166 & $\mathrm{H}$ & 11.3650 & 6.3280 & -0.8118 \\
\hline $\mathrm{H}$ & 7.3594 & -5.1890 & -1.5276 & $\mathrm{H}$ & -10.3268 & 2.4186 & -5.1741 \\
\hline H & 8.5664 & -4.2929 & -3.4646 & $\mathrm{H}$ & -8.9857 & 1.3474 & -5.6116 \\
\hline $\mathrm{H}$ & 8.2205 & -2.0033 & 0.1485 & $\mathrm{H}$ & -0.6693 & 1.6437 & 6.2568 \\
\hline H & 9.3339 & -2.2648 & -4.6734 & $\mathrm{H}$ & -2.2659 & 2.2805 & 5.8191 \\
\hline $\mathrm{H}$ & 8.9476 & 0.0247 & -1.0525 & $\mathrm{H}$ & 5.9637 & 7.5730 & 2.8312 \\
\hline H & 9.5102 & -0.0931 & -3.4717 & $\mathrm{H}$ & 4.4369 & 7.8942 & 3.6683 \\
\hline C & -2.8067 & 0.8709 & 0.4823 & $\mathrm{H}$ & -0.3133 & -1.3602 & 7. 3437 \\
\hline C & -1.6111 & 1.2573 & -0.0716 & $\mathrm{H}$ & 0.7307 & -0.9027 & 8.6828 \\
\hline C & -1.6219 & 2.5203 & -0.7613 & $\mathrm{H}$ & 1.4732 & -3.0002 & 6.5433 \\
\hline $\mathrm{N}$ & -0.5230 & 3.1181 & -1.1798 & $\mathrm{H}$ & 0.8204 & -3.4282 & 8.1477 \\
\hline $\mathrm{N}$ & -2.8022 & 3.1545 & -1.0188 & $\mathrm{H}$ & 2.4050 & -2.6533 & 7.9936 \\
\hline C & -3.9216 & 2.6893 & -0.5013 & $\mathrm{H}$ & 8.8027 & -6.2362 & 0.2306 \\
\hline $\mathrm{N}$ & -3.9476 & 1.5952 & 0.2982 & $\mathrm{H}$ & 9.7984 & -5.6286 & -1.1047 \\
\hline S & 1.8518 & -0.7163 & -2.8946 & $\mathrm{H}$ & 4.6462 & -6.6471 & -1.9064 \\
\hline C & 1.4263 & 0.3142 & -1.5916 & $\mathrm{H}$ & 0.7847 & -7.3756 & -5.0505 \\
\hline C & -5.1972 & 3.3838 & -0.8287 & $\mathrm{H}$ & -0.9610 & -7.6086 & -5.2528 \\
\hline $\mathrm{N}$ & 0.2743 & -0.1316 & -1.0612 & $\mathrm{H}$ & 4.4649 & -3.4721 & -4.9969 \\
\hline C & -0.3005 & -1.2921 & -1.6452 & $\mathrm{H}$ & 1.6714 & 1.8000 & 5.7447 \\
\hline C & -1.5957 & -1.8591 & -1.1679 & $\mathrm{H}$ & -4.8625 & 1.3370 & 0.8623 \\
\hline C & 0.4539 & -1.7445 & -2.6833 & C & 1.8718 & 6.7668 & -2.9188 \\
\hline C & 0.2060 & -2.9062 & -3.5921 & C & 0.5502 & 6.5299 & -2.2343 \\
\hline C & -0.3329 & 0.4849 & 0.1630 & 0 & 0.4194 & 5.6644 & -1.3698 \\
\hline H & 0.4331 & 2.8939 & -0.7962 & $\mathrm{~N}$ & -0.4905 & 7. 3134 & -2.6450 \\
\hline H & -0.5940 & 4.0862 & -1.4828 & C & -1.8570 & 6.8977 & -2.3648 \\
\hline H & -0.5334 & -0.3336 & 0.8550 & C & -2.3989 & 6.1944 & -3.6103 \\
\hline H & 0.4440 & 1.1232 & 0.5848 & 0 & -2.3171 & 6.7609 & -4.7030 \\
\hline H & -5.8735 & 3.3737 & 0.0320 & $\mathrm{~N}$ & -2.9101 & 4.9603 & -3.4288 \\
\hline $\mathrm{H}$ & -4.9811 & 4.4026 & -1.1510 & C & -3.4722 & 4.2180 & -4.5463 \\
\hline H & -5.6939 & 2.8515 & -1.6464 & C & -3.3356 & 2.7077 & -4.3747 \\
\hline H & -2.9179 & -0.0205 & 1.0924 & C & -1.8983 & 2.1704 & -4.4262 \\
\hline H & -1.6226 & -1.9757 & -0.0840 & C & -1.8568 & 0.7180 & -3.9470 \\
\hline $\mathrm{H}$ & -1.7444 & -2.8489 & -1.6015 & C & -1.3006 & 2.2892 & -5.8332 \\
\hline H & -2.4377 & -1.2405 & -1.4838 & C & 2.6982 & 2.7126 & -2.4165 \\
\hline $\mathrm{H}$ & 1.0557 & -3.5982 & -3.5959 & C & 3.7868 & 3.5937 & -2.5416 \\
\hline $\mathrm{H}$ & 0.0467 & -2.5729 & -4.6235 & C & 4.0472 & 4.2520 & -3.7408 \\
\hline C & 1.3893 & -2.6801 & 7.5844 & C & 3.1935 & 4.0836 & -4.8317 \\
\hline C & 0.7379 & -1.2982 & 7.6632 & C & 2.0587 & 3.2873 & -4.6898 \\
\hline C & 1.4214 & -0.2821 & 6.7411 & C & 1.8132 & 2.6109 & -3.4930 \\
\hline 0 & 1.7935 & -0.7120 & 5.6068 & C & 2.5159 & 2.0066 & -1.0682 \\
\hline 0 & 1.5460 & 0.9059 & 7.1498 & 0 & 1.8281 & 2.6187 & -0.1973 \\
\hline C & -8.7493 & 2.5329 & 3.7275 & $\mathrm{H}$ & 1.9756 & 7.7880 & -3.2961 \\
\hline C & -7.7501 & 1.4512 & 3.3112 & $\mathrm{H}$ & 2.6781 & 6.5384 & -2.2225 \\
\hline C & -6.8666 & 1.8313 & 2.1157 & $\mathrm{H}$ & -1.8443 & 6.2661 & -1.4767 \\
\hline 0 & -7.0328 & 2.9546 & 1.5822 & $\mathrm{H}$ & -2.4789 & 7.7763 & -2.1669 \\
\hline 0 & -6.0065 & 0.9562 & 1.7596 & $\mathrm{H}$ & -3.8036 & 2.4096 & -3.4322 \\
\hline H & -9.3390 & 2.2042 & 4.5909 & $\mathrm{H}$ & -3.9272 & 2.2250 & -5.1635 \\
\hline $\mathrm{H}$ & -8.2350 & 3.4572 & 3.9972 & $\mathrm{H}$ & -1.2865 & 2.7678 & -3.7397 \\
\hline H & -8.2651 & 0.5161 & 3.0552 & $\mathrm{H}$ & -0.3071 & 1.8318 & -5.8756 \\
\hline H & -7.0759 & 1.1943 & 4.1370 & $\mathrm{H}$ & -1.9315 & 1.7691 & -6.5649 \\
\hline C & -1.2939 & 1.9724 & 5.4216 & $\mathrm{H}$ & -1.2046 & 3.3311 & -6.1537 \\
\hline C & -0.6254 & 3.1319 & 4.6631 & $\mathrm{H}$ & -2.4378 & 0.0673 & -4.6121 \\
\hline C & 0.6810 & 2.7504 & 4.0433 & $\mathrm{H}$ & -2.2759 & 0.6161 & -2.9428 \\
\hline C & 1.1387 & 2.8162 & 2.7456 & $\mathrm{H}$ & -0.8324 & 0.3368 & -3.9283 \\
\hline $\mathrm{N}$ & 1.7080 & 2.1767 & 4.7680 & $\mathrm{H}$ & -2.9759 & 4.5728 & -5.4528 \\
\hline C & 2.7110 & 1.8985 & 3.9112 & $\mathrm{H}$ & 4.4492 & 3.7604 & -1.7000 \\
\hline $\mathrm{N}$ & 2.4120 & 2.2777 & 2.6742 & $\mathrm{H}$ & 4.9122 & 4.9042 & -3.8166 \\
\hline $\mathrm{H}$ & -1.4554 & 1.1146 & 4.7607 & $\mathrm{H}$ & 3.3959 & 4.5883 & -5.7717 \\
\hline $\mathrm{H}$ & -1.2842 & 3.4916 & 3.8651 & $\mathrm{H}$ & 1.3623 & 3.1832 & -5.5141 \\
\hline H & -0.4828 & 3.9774 & 5.3502 & $\mathrm{H}$ & 0.9278 & 1.9976 & -3.4010 \\
\hline $\mathrm{H}$ & 0.6598 & 3.2249 & 1.8701 & C & -5.3059 & -3.3444 & 3.4682 \\
\hline $\mathrm{H}$ & 3.6143 & 1.3912 & 4.2067 & C & -3.9473 & -2.7335 & 3.8469 \\
\hline $\mathrm{H}$ & 3.1097 & 1.6251 & 1.4005 & 0 & -3.8825 & -1.5897 & 4.2893 \\
\hline C & 4.9522 & 7.1885 & 3.0084 & C & -6.1471 & -2.3526 & 2.6427 \\
\hline C & 4.9964 & 5.7877 & 3.6256 & C & -5.6779 & -2.2335 & 1.2082 \\
\hline C & 5.7632 & 4.7236 & 2.8161 & $\mathrm{~N}$ & -6.4900 & -2.7678 & 0.2595 \\
\hline C & 5.8906 & 3.4477 & 3.6611 & 0 & -4.6027 & -1.6943 & 0.9030 \\
\hline C & 5.0951 & 4.4222 & 1.4661 & $\mathrm{~N}$ & -2.8477 & -3.5302 & 3.7530 \\
\hline $\mathrm{H}$ & 4.4245 & 7. 1914 & 2.0492 & C & -1.5446 & -3.0536 & 4.2014 \\
\hline $\mathrm{H}$ & 3.9713 & 5.4254 & 3.7842 & C & -0.8015 & -2.2765 & 3.1090 \\
\hline H & 5.4520 & 5.8554 & 4.6229 & 0 & -1.0919 & -2.4161 & 1.9120 \\
\hline $\mathrm{H}$ & 6.7756 & 5.1015 & 2.6127 & C & -0.7765 & -4.3687 & 4.4519 \\
\hline $\mathrm{H}$ & 4.9173 & 3.1744 & 4.0753 & C & -1.2869 & -5.2639 & 3.3100 \\
\hline $\mathrm{H}$ & 6.2480 & 2.5963 & 3.0774 & C & -2.7652 & -4.8601 & 3.1371 \\
\hline H & 6.5793 & 3.5974 & 4.5001 & $\mathrm{~N}$ & 0.2419 & -1.5568 & 3.5481 \\
\hline $\mathrm{H}$ & 4.0936 & 4.0017 & 1.6045 & C & 1.3350 & -1.1901 & 2.6842 \\
\hline H & 5.6998 & 3.7001 & 0.9060 & C & 2.6156 & -1.9155 & 3.1217 \\
\hline $\mathrm{H}$ & 5.0043 & 5.3220 & 0.8476 & 0 & 2.6118 & -3.0027 & 3.6903 \\
\hline
\end{tabular}

\begin{tabular}{|c|c|c|c|}
\hline & 368 & -1.2375 & 2.7675 \\
\hline & 880 & -1.6236 & 3.1220 \\
\hline & & -0.9961 & \\
\hline & & 332 & كוt \\
\hline & 801 & -1.2553 & 012 \\
\hline & 6.0952 & 0.1410 & 17205 \\
\hline & 297 & & \\
\hline & 5.0057 & 30 & \\
\hline & -7.1967 & 566 & 5597 \\
\hline & -6.0466 & & \\
\hline & & & \\
\hline & -1.6 & -4 & 1287 \\
\hline & & & \\
\hline & -1. & & \\
\hline & -0 . & & \\
\hline & -3.2 & -5 & \\
\hline & -3. & & 792 \\
\hline & -1 & & \\
\hline & & & \\
\hline & & -1 . & \\
\hline & & & \\
\hline & & & \\
\hline & & & \\
\hline & & -2 . & \\
\hline & & & \\
\hline & & & \\
\hline & -4 & & -4 \\
\hline & -2 . & & \\
\hline & -0 . & & \\
\hline & & & \\
\hline & & & \\
\hline y & & & \\
\hline & & & \\
\hline & $-7.2 .3 x$ & -3 & \\
\hline † & -6 & -2 & \\
\hline H & & & \\
\hline & -5 . & & \\
\hline & -10 & & \\
\hline C & -9 & -2 & 91 \\
\hline 0 & -8 & & \\
\hline C & -11. & & \\
\hline & -10. & -4 & -0. \\
\hline 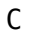 & & & 31 \\
\hline & -9 . & & \\
\hline & -9 & & \\
\hline & -9 & & \\
\hline & & & -0. \\
\hline & -9 . & & \\
\hline 4 & -10.6 & -3 & \\
\hline t & -11. & -5 & \\
\hline $\mathrm{H}$ & -12.1 & -3 & \\
\hline & -11. & & \\
\hline 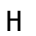 & -9. & -2 & -1 \\
\hline t & -8.3 & & \\
\hline I & -10 & & \\
\hline & -8 & & \\
\hline H & -8.5 & & -1 . \\
\hline 4 & -10.2 & & \\
\hline & & & \\
\hline I & -8. & & \\
\hline 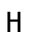 & -10 & -6 & \\
\hline H & -10.3 & -3 & -2 \\
\hline & & & \\
\hline & -11 & -4 & \\
\hline 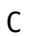 & & -1 . & \\
\hline C & & -2 & \\
\hline & & -1 & \\
\hline & & & \\
\hline C & & & \\
\hline C & & -0. & \\
\hline 11 & & & \\
\hline & & -3 & \\
\hline t & & & \\
\hline 11 & & & -2 \\
\hline 11 & & & \\
\hline & & & \\
\hline & & & \\
\hline 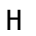 & 1.8 & 1. & \\
\hline & 4.516 & 2.231 & -0.4 \\
\hline
\end{tabular}




\section{EP(BA)-R}

\begin{tabular}{|c|c|c|c|}
\hline & 11.1675 & 5.0766 & -0.0552 \\
\hline & 10.0162 & 332 & 0.9597 \\
\hline & 9.4585 & 3.7867 & 148 \\
\hline & 8.1811 & 3116 & 1100 \\
\hline & 10.2156 & 2.8267 & 1.9620 \\
\hline & 9.3963 & 1.8038 & 2.1464 \\
\hline & 8.1532 & 2.0435 & 1.6481 \\
\hline & 11.9683 & 4.4353 & 0.323 \\
\hline & 9.2052 & 57 & \\
\hline & 10.3751 & 5.6219 & 1.8750 \\
\hline & 7.3098 & 3.7623 & 0.6638 \\
\hline & 9.6396 & 0.8811 & 2. \\
\hline & 7.3474 & 1.4064 & 1.6707 \\
\hline & -9.7040 & 2.4789 & -4.3277 \\
\hline & -8.7452 & 3.2011 & -3.3628 \\
\hline & -7.6865 & 2.26 & -2.8278 \\
\hline & -6.5913 & 1.8991 & -3.6239 \\
\hline & -7.8035 & 1.67 & -1 \\
\hline & -5.6756 & 0.9427 & -3.1 \\
\hline & -6.8961 & 0.7072 & -1 \\
\hline & -5.8385 & 0.3211 & -1 \\
\hline & -4.9518 & -0.6556 & -1.6317 \\
\hline & -10.2101 & 1.6521 & -3.8187 \\
\hline & -9.3164 & 3. & -2 . \\
\hline & -8.2743 & 4.0418 & -3 \\
\hline & -6.4593 & 2.3645 & -4.5980 \\
\hline & -8.6149 & 1.97 & -0 \\
\hline & -4.8300 & 0.6574 & -3 \\
\hline & -6.9802 & 0.2992 & -0 . \\
\hline & -4.9646 & -0.9 & -0 . \\
\hline & -0.6309 & -6.4233 & -5 \\
\hline & -0.8905 & -6.5611 & -3 \\
\hline & -2.2302 & -6 . & -3 \\
\hline & -2.4894 & -6.0569 & -2 . \\
\hline & -3.2327 & -5.5556 & -4.2696 \\
\hline & -3.6892 & -5.5 & -1.5 \\
\hline & -4.4392 & -5 & -3.7 \\
\hline & -4.6738 & -5.0801 & -2.3865 \\
\hline & -5.8760 & -4.6049 & -1 \\
\hline & -0.6862 & -5.3 & -5 \\
\hline & -0.7839 & -7.6175 & -3.6552 \\
\hline & -0.0971 & -6.0354 & -3.3896 \\
\hline & -1.7320 & -6.4285 & -1.3659 \\
\hline & -3.0738 & -5.5258 & -5.3424 \\
\hline & -3.8521 & -5.5789 & -0.2 \\
\hline & -5.2033 & -4.6749 & -4 \\
\hline & -5.8585 & -4.4913 & -0.9782 \\
\hline & 4.6320 & -4.0714 & -5.6607 \\
\hline & 4.8026 & -4.3 & -4 \\
\hline & 5.7411 & -3.8728 & -3.5443 \\
\hline & 4.5274 & -2.5588 & -5 \\
\hline & 5.5 & -4.2 & -6 \\
\hline & 5.3985 & -2.0522 & -5.4944 \\
\hline & 3.633 & -2.1431 & -5.439 \\
\hline & 4.2 & -2 & -6 \\
\hline & 3.8383 & -5.1220 & -3.6438 \\
\hline & 3.9230 & -5.5874 & -2.2828 \\
\hline & 2.5 & -5 & -1 \\
\hline & 2.0108 & -4.1247 & -1.3150 \\
\hline & 0.6857 & -4.2454 & -0 . \\
\hline & 2. & -3 & \\
\hline & 3.1185 & -5.4963 & -4.2427 \\
\hline & 4.6832 & -4.9758 & -1.7966 \\
\hline & 2.7 & -5 & -0. \\
\hline & 1.8564 & -6.1552 & -2 \\
\hline & 1.8132 & -3.6778 & -2 \\
\hline & 0.8400 & -4.7197 & 0.4169 \\
\hline & 0.2322 & -3.2749 & -0.3699 \\
\hline & -0.0431 & -4.8401 & -1.1200 \\
\hline & 3.9392 & -3.1050 & -1.0833 \\
\hline & 3.1780 & -3.5914 & 0.4403 \\
\hline & 2.5639 & -2.2006 & -0.4665 \\
\hline & 8.8440 & -5.5576 & -0.9678 \\
\hline & 8.1985 & -4.1618 & -0.9946 \\
\hline & 9.2 & -3.0499 & 671 \\
\hline & 10.0 & -2.8977 & -2 . \\
\hline & & -2 & \\
\hline
\end{tabular}

C $\quad \begin{array}{llll}0.9842 & -1.8897 & -2.2796\end{array}$

$\begin{array}{llll}\text { C } 10.3536 & -1.1355 & -0.0779\end{array}$

C $11.1524-1.0031-1.2130$

$\begin{array}{lll}9.5026 & -5.6687 & -0.1004\end{array}$

$\begin{array}{lll}7.5857 & -4.0265 & -0.0964\end{array}$

$\begin{array}{lll}7.5139 & -4.0968 & -1.8483\end{array}$

$\begin{array}{lll}9.8866 & -3.5735 & -3.0473\end{array}$

$\begin{array}{lll}8.7811 & -2.2471 & 0.8787\end{array}$

$\begin{array}{lll}11.5947 & -1.7887 & -3.1729\end{array}$

$\begin{array}{lll}10.4579 & -0.4326 & 0.7419\end{array}$

$\begin{array}{lll}11.8891 & -0.2077 & -1.2706\end{array}$

$\begin{array}{lll}-3.0848 & 0.7372 & 0.5850\end{array}$

$\begin{array}{lll}-1.8924 & 0.9356 & -0.0566\end{array}$

$\begin{array}{lll}-1.7044 & 2.2153 & -0.6787\end{array}$

$\begin{array}{llll}\mathrm{N} & -0.5411 & 2.5895 & -1.1996\end{array}$

$\begin{array}{llll}\mathrm{N} & -2.7341 & 3.1073 & -0.7269\end{array}$

$\begin{array}{llll}C & -3.8867 & 2.8093 & -0.1483\end{array}$

$\begin{array}{llll}\mathrm{N} & -4.0744 & 1.6682 & 0.5475\end{array}$

$\begin{array}{lll}1.0273 & -0.7657 & -3.3595\end{array}$

$\begin{array}{lll}0.7378 & 0.0488 & -1.8649\end{array}$

$\begin{array}{lll}-5.0380 & 3.7455 & -0.3009\end{array}$

$\begin{array}{llll}\mathrm{N} & -0.3328 & -0.5545 & -1.3159\end{array}$

$\begin{array}{llll}\text { C } & -0.9632 & -1.6156 & -2.0074\end{array}$

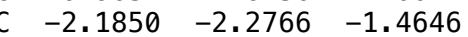

C $\quad-0.3190-1.8754-3.1760$

$\begin{array}{llll}\text { C } & -0.6121 & -2.9195 & -4.2019\end{array}$

$\begin{array}{llll}C & -0.7903 & -0.0812 & 0.0119\end{array}$

$\begin{array}{rrrr}\text { H } & 0.2289 & 1.9170 & -1.3072\end{array}$

$\begin{array}{llll}H & -0.4071 & 3.5402 & -1.5425\end{array}$

$\begin{array}{llll}\mathrm{H} & -1.0986 & -0.9422 & 0.6059\end{array}$

$\begin{array}{llll}H & 0.0892 & 0.3614 & 0.4820\end{array}$

$\begin{array}{llll}H & -5.6589 & 3.7376 & 0.5990\end{array}$

$\begin{array}{llll}\mathrm{H} & -4.6772 & 4.7479 & -0.5304\end{array}$

$\begin{array}{llll}H & -5.6605 & 3.4000 & -1.1347\end{array}$

$\begin{array}{llll}\mathrm{H} & -3.3075 & -0.1616 & 1.1503\end{array}$

$\begin{array}{llll}H & -2.0701 & -2.5601 & -0.4159\end{array}$

H $\quad-2.4016-3.1810-2.0321$

H $\quad-3.0450-1.6115-1.5540$

$0.2203-3.6280-4.2886$

$\begin{array}{lll}-0.7699 & -2.4742 & -5.1905\end{array}$

$\begin{array}{llll}\text { C } & 1.3823 & -3.3648 & 7.3088\end{array}$

$\begin{array}{llll}\text { C } & 0.6327 & -2.0345 & 7.4022\end{array}$

$\begin{array}{llll}\text { C } & 1.3870 & -0.9191 & 6.6700\end{array}$

$\begin{array}{llll}\text { C } & 1.6106 & -1.1445 & 5.4117\end{array}$

$\begin{array}{llll}0 & 1.7329 & 0.1026 & 7.2773\end{array}$

$\begin{array}{lrrr}\text { C } & -8.6529 & 2.5002 & 4.1876\end{array}$

$\begin{array}{llll}C & -7.7483 & 1.4122 & 3.6112\end{array}$

$\begin{array}{llll}\text { C } & -6.8549 & 1.8833 & 2.4594\end{array}$

$\begin{array}{llll}0 & -6.8856 & 3.0896 & 2.1217\end{array}$

$\begin{array}{llll}0 & -6.1168 & 0.9818 & 1.9310\end{array}$

$\begin{array}{llll}\text { H } & -9.2367 & 2.1142 & 5.0310\end{array}$

$\begin{array}{llll}\mathrm{H} & -8.0641 & 3.3497 & 4.5386\end{array}$

$\begin{array}{llll}\mathrm{H} & -8.3337 & 0.5573 & 3.2497\end{array}$

$\begin{array}{llll}\mathrm{H} & -7.0810 & 1.0037 & 4.3802\end{array}$

$\begin{array}{llll}\text { C } & -1.1850 & 1.5471 & 5.6338\end{array}$

$\begin{array}{llll}C & -1.0973 & 1.1856 & 4.1398\end{array}$

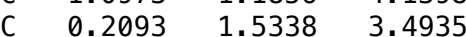

$\begin{array}{llll}\text { C } & 0.5257 & 2.1924 & 2.3202\end{array}$

$\mathrm{N} \quad 1.4248 \quad 1.1463 \quad 4.0202$

$\begin{array}{llll}C & 2.3962 & 1.5503 & 3.1670\end{array}$

$\begin{array}{llll}\mathrm{N} & 1.8948 & 2.1882 & 2.1163\end{array}$

$\begin{array}{llll}\mathrm{H} & -0.3754 & 1.0939 & 6.2114\end{array}$

$\begin{array}{llll}\mathrm{H} & -1.2867 & 0.1118 & 4.0198\end{array}$

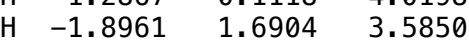

$\begin{array}{llll}\mathrm{H} & -0.1414 & 2.6792 & 1.6244\end{array}$

$\begin{array}{llll}\mathrm{H} & 3.4420 & 1.3512 & 3.3440\end{array}$

$\begin{array}{llll}H & 3.0298 & 1.6763 & 0.6347\end{array}$

$\begin{array}{llll}\text { C } & 5.1833 & 6.7495 & 3.5301\end{array}$

$\begin{array}{llll}\text { C } & 5.1145 & 5.4064 & 4.2619\end{array}$

$\begin{array}{llll}\text { C } \quad 5.6472 & 4.2047 & 3.4614\end{array}$

$\begin{array}{lll}\text { C } \quad 5.6312 & 2.9421 & 4.3340\end{array}$

$\begin{array}{llll}\text { C } & 4.8633 & 3.9925 & 2.1590\end{array}$

$\begin{array}{llll}\mathrm{H} & 4.5420 & 6.7596 & 2.6434\end{array}$

$\begin{array}{llll}\mathrm{H} & 4.0742 & 5.2011 & 4.5506\end{array}$

$\begin{array}{llll}\text { H } & 5.6817 & 5.4798 & 5.1994\end{array}$

$\begin{array}{llll}\mathrm{H} & 6.6937 & 4.4131 & 3.1958\end{array}$

$\begin{array}{llll}\mathrm{H} & 4.6193 & 2.7302 & 4.6969\end{array}$

$\begin{array}{llll}\mathrm{H} & 5.9794 & 2.0645 & 3.7823\end{array}$

$\begin{array}{llll}H & 6.2737 & 3.0652 & 5.2129\end{array}$

$\begin{array}{llll}\mathrm{H} & 3.7899 & 3.8870 & 2.3405\end{array}$

$\begin{array}{llll}H & 3.7899 & 3.8870 & 2.3405 \\ \mathrm{H} & 5.1858 & 3.0796 & 1.6508\end{array}$

\begin{tabular}{|c|c|c|c|}
\hline & 5.0011 & 4.8225 & 1.4574 \\
\hline & 5065 & -3.4856 & -3.9410 \\
\hline & -9.3487 & 690 & 4325 \\
\hline & 10.8263 & 558 & 007 \\
\hline & 11.5800 & 6.0732 & -0.2478 \\
\hline & -10.4671 & 3.1607 & -4.7183 \\
\hline & -9.1539 & 34 & -5 . \\
\hline & -1.1194 & & \\
\hline & -2.1392 & 1.2045 & 6.045 \\
\hline & & & \\
\hline & 4.8617 & & \\
\hline & -0.3576 & -2 & \\
\hline & 0.2 & -1 & \\
\hline & & & \\
\hline & 0.8 & & \\
\hline & 2.3404 & -3 & 7. \\
\hline & 8.0797 & -6 & -0.9205 \\
\hline & & & \\
\hline & 4.2 & -6 . & \\
\hline & 0.3657 & -6 & -5 . \\
\hline & -1.3561 & -6 & \\
\hline & 3.7 & -4 & \\
\hline & 1.5 & & \\
\hline & -5.0203 & & 1. \\
\hline & & & \\
\hline & 0. & & \\
\hline & 0.2 & & -2 \\
\hline & -0. & & -1 \\
\hline & -1. & & \\
\hline & -2 & & -2 \\
\hline & $-1 . \varsigma$ & & -3 \\
\hline & -2 & & \\
\hline & -3 & & \\
\hline & -2 & & -4 \\
\hline & -1.4 & & -4 \\
\hline & -0. & & -4 \\
\hline & -1 & & \\
\hline & 3. & & -3 \\
\hline & 2.5 & & -3 \\
\hline & & & \\
\hline & & & \\
\hline & 3.2 & & -5 \\
\hline & 3.6 & & -4 \\
\hline & & & \\
\hline & 2. & & -1 \\
\hline & 2.0 & & -1 . \\
\hline & 2.7 & & -1 \\
\hline & -1 . & & \\
\hline & -2 & & -0 \\
\hline & -3.1 & & -3 \\
\hline & -3.5 & & -5 \\
\hline & & & \\
\hline & -0. & & \\
\hline & -1.7 & & -6 . \\
\hline & -1.2 & & -5 . \\
\hline & & & \\
\hline & -1 & & \\
\hline & 0 & & -5 \\
\hline & -3.2 & & -4 \\
\hline & & & \\
\hline & 1 & & -5 \\
\hline & 2.8 & & -7 \\
\hline & & & \\
\hline & & & \\
\hline & -5.2 & -3 & \\
\hline & -4.6 & -2 & \\
\hline & -3 & & \\
\hline & & & \\
\hline & -5. & & \\
\hline & -6 & -2 & \\
\hline & -4 . & & \\
\hline & -2 & & \\
\hline & -1.6 & & \\
\hline & & & \\
\hline & -1.2 & & \\
\hline & -0.5 & & \\
\hline & -1.5 & & \\
\hline & & & \\
\hline & 0.4 & -1 . & . \\
\hline & & & \\
\hline & & & ? \\
\hline
\end{tabular}

S21 


\begin{tabular}{|c|c|c|c|}
\hline & 828 & -3.3817 & 09 \\
\hline & 177 & -1.3918 & 1107 \\
\hline & 22 & 82 & \\
\hline & 106 & & \\
\hline & & -1.4302 & 609 \\
\hline & & -10583 & \\
\hline & UL & 0.1 & \\
\hline & & -0. & \\
\hline & 707 & -0. & 04. \\
\hline$H_{2}$ & & & \\
\hline & -6 . & -1. & \\
\hline & & -4 . & \\
\hline & 143 & -4 & \\
\hline & & & \\
\hline & -1.2 & & \\
\hline & -1. & -5 & \\
\hline & -3 & -5 . & \\
\hline & -3 & & \\
\hline & -1 & -2 & \\
\hline & 1.3010 & -0. & \\
\hline & & -2 . & \\
\hline & & & \\
\hline & & -1 . & \\
\hline & 5. & & $0<1$ \\
\hline & & & \\
\hline & & & \\
\hline 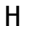 & 2. & & -3 \\
\hline & -4 & & \\
\hline & & & \\
\hline & -0 . & & \\
\hline & 5. & -1 & \\
\hline & & -0. & \\
\hline & & & \\
\hline & & -1 & \\
\hline$H$ & -7 . & -3 & \\
\hline & & -2 & \\
\hline & & & \\
\hline & -5.8 & -3. & \\
\hline & -11 . & - & \\
\hline & -10 & & \\
\hline & -9. & & \\
\hline & -11. & -4 . & \\
\hline & -10 & -4 & \\
\hline & & & \\
\hline & -9. & & \\
\hline & -9. & & \\
\hline & -8 . & & \\
\hline & & & \\
\hline 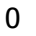 & -9. & & \\
\hline & -10 & -3 & \\
\hline & -12 & -4 & \\
\hline & & & \\
\hline & -12 & -5 . & \\
\hline & -9. & -2.2 & -1 \\
\hline & & & \\
\hline & & & \\
\hline & -7 & & \\
\hline & -8 . & & \\
\hline & & & \\
\hline & -9. & & \\
\hline & -8 . & & \\
\hline & -10 . & - & \\
\hline & & & \\
\hline & -9. & & \\
\hline & -11. & -4 & \\
\hline & 7 & -0. & \\
\hline & & & \\
\hline & & -0 . & \\
\hline & & & \\
\hline & & & \\
\hline & & & \\
\hline & & & \\
\hline & & & \\
\hline & & & 2. \\
\hline & & & \\
\hline & & & \\
\hline & & & \\
\hline & & 178 & 1 \\
\hline & & D. & 070 \\
\hline
\end{tabular}

\section{EP(BA)-S}

C $11.3111 \quad 4.9426 \quad-0.5298$

$\begin{array}{llll}\text { C } & 9.8384 & 4.5912 & -0.7990\end{array}$

$\begin{array}{llll}C & 9.3004 & 3.5308 & 0.1189\end{array}$

$\begin{array}{llll}C & 8.5753 & 2.3965 & -0.1853\end{array}$

$\begin{array}{llll}\mathrm{N} & 9.4756 & 3.6187 & 1.4876\end{array}$

$\begin{array}{llll}C & 8.8684 & 2.5569 & 1.9893\end{array}$

$\begin{array}{llll}\mathrm{N} & 8.3106 & 1.7793 & 1.0227\end{array}$

$\begin{array}{lrrr}\mathrm{H} & 11.4365 & 5.2321 & 0.5163\end{array}$

$\begin{array}{llll}H & 9.7078 & 4.2667 & -1.8388\end{array}$

$\begin{array}{llll}H & 9.2362 & 5.5036 & -0.6837 \\ H & 8.2467 & 1.9702 & -1.1201\end{array}$

$\begin{array}{rrrr}\mathrm{H} & 8.2467 & 1.9702 & -1.1201 \\ \mathrm{H} & 8.7985 & 2.3039 & 3.0371\end{array}$

$\begin{array}{llll}\text { C } & -9.5298 & 2.0534 & -4.7641\end{array}$

$\begin{array}{llll}C & -8.3673 & 2.6721 & -3.9696\end{array}$

$\begin{array}{llll}\text { C } & -7.4647 & 1.6214 & -3.3640\end{array}$

$\begin{array}{llll}\text { C } & -6.6558 & 0.8205 & -4.1838\end{array}$

$\begin{array}{llll}C & -7.4380 & 1.3784 & -1.9866\end{array}$

$\begin{array}{llll}\text { C } & -5.8632 & -0.1914 & -3.6548\end{array}$

$\begin{array}{llll}\text { C } & -6.6500 & 0.3658 & -1.4362\end{array}$

$\begin{array}{llll}\text { C } & -5.8585 & -0.4266 & -2.2741\end{array}$

$\begin{array}{llll}0 & -5.0801 & -1.4455 & -1.8145\end{array}$

H $-10.1453 \quad 1.4221 \quad-4.1151$

$\begin{array}{llll}\mathrm{H} & -8.7682 & 3.3118 & -3.1747\end{array}$

$\begin{array}{llll}\mathrm{H} & -7.7880 & 3.3260 & -4.6347\end{array}$

$\begin{array}{llll}H & -6.6432 & 0.9969 & -5.2569\end{array}$

$\begin{array}{llll}\mathrm{H} & -8.0279 & 1.9961 & -1.3145\end{array}$

H $\quad-5.2286 \quad-0.8027 \quad-4.2872$

$\begin{array}{llll}H & -6.6332 & 0.2291 & -0.3607\end{array}$

$\begin{array}{llll}\text { H } & -4.9467 & -1.4032 & -0.8306\end{array}$

$\begin{array}{llll}\text { C } & -0.5504 & -7.0108 & -4.6382\end{array}$

$\begin{array}{lll}-0.8312 & -6.9465 & -3.1348\end{array}$

$\begin{array}{llll}\text { C } & -2.1796 & -6.3840 & -2.7175\end{array}$

$\begin{array}{llll}\text { C } & -2.4969 & -6.3005 & -1.3536\end{array}$

$\begin{array}{llll}\text { C } & -3.1360 & -5.9210 & -3.6292\end{array}$

$\begin{array}{llll}\text { C } & -3.7128 & -5.7845 & -0.9140\end{array}$

$\begin{array}{llll}\text { C } & -4.3604 & -5.4036 & -3.2060\end{array}$

$\begin{array}{llll}\text { C } & -4.6577 & -5.3375 & -1.8442\end{array}$

$\begin{array}{llll}0 & -5.8863 & -4.8623 & -1.4769\end{array}$

H $\quad-0.5817-6.0163-5.0950$

$\begin{array}{llll}\mathrm{H} & -0.7251 & -7.9534 & -2.7094\end{array}$

$\begin{array}{llll}\mathrm{H} & -0.0461 & -6.3484 & -2.6553\end{array}$

H $\quad-1.7759-6.6431 \quad-0.6169$

$\begin{array}{llll}H & -2.9291 & -5.9568 & -4.6935\end{array}$

$\begin{array}{llll}H & -3.9285 & -5.7270 & 0.1503\end{array}$

$\begin{array}{lll}-5.0922 & -5.0406 & -3.9193\end{array}$

$\begin{array}{lll}-5.9055 & -4.7043 & -0.5207\end{array}$

$\begin{array}{lll}4.7433 & -4.7580 & -5.0660\end{array}$

$\begin{array}{lll}4.9711 & -5.3916 & -3.7127\end{array}$

$\begin{array}{lll}5.9765 & -6.0438 & -3.4342\end{array}$

$6.0466-4.5195 \quad-5.8264$

$\begin{array}{lll}4.0905 & -5.4301 & -5.6392\end{array}$

$\begin{array}{lll}6.6129 & -5.4501 & -5.9028\end{array}$

$\begin{array}{lll}6.6785 & -3.7954 & -5.3044\end{array}$

$\begin{array}{lll}5.8473 & -4.1387 & -6.8320\end{array}$

$\begin{array}{lll}3.9407 & -5.2285 & -2.8477\end{array}$

$3.9696-5.8392-1.5386$

$\begin{array}{lll}2.5933 & -5.8400 & -0.8768\end{array}$

$\begin{array}{lll}1.9983 & -4.4527 & -0.5780\end{array}$

$\begin{array}{lll}0.5880 & -4.5975 & 0.0030\end{array}$

$\begin{array}{lll}2.8800 & -3.6364 & 0.3695\end{array}$

$\begin{array}{lll}3.1869 & -4.6008 & -3.0814\end{array}$

$\begin{array}{lll}4.7013 & -5.3300 & -0.9007\end{array}$

$\begin{array}{lll}2.6785 & -6.3907 & 0.0685\end{array}$

$1.8964-6.4102-1.5062$

$\begin{array}{lll}1.9060 & -3.8910 & -1.5205\end{array}$

$\begin{array}{lll}0.6045 & -5.2648 & 0.8739\end{array}$

$\begin{array}{lll}0.1861 & -3.6423 & 0.3433\end{array}$

$\begin{array}{lll}-0.1091 & -5.0137 & -0.7296\end{array}$

$\begin{array}{lll}3.9238 & -3.5925 & 0.0499\end{array}$

$\begin{array}{lll}2.8514 & -4.0412 & 1.3848\end{array}$

$\begin{array}{lll}2.5199 & -2.6071 & 0.4181\end{array}$

$\begin{array}{lll}8.8721 & -5.6983 & -0.1634\end{array}$

$\begin{array}{lll}7.7377 & -4.7846 & -0.6642\end{array}$

$8.2794-3.5833-1.4031$

$8.5604-3.6681-2.7736$

$\begin{array}{lll}8.5805 & -2.3932 & -0.7297\end{array}$

$\begin{array}{llll}9.1534 & -2.5984 & -3.4448\end{array}$

\begin{tabular}{|c|c|c|c|}
\hline & 9.1667 & -1.3188 & -1.3981 \\
\hline & 9.4650 & -1.4237 & -2.7573 \\
\hline & 9.5381 & -5.1578 & 0.5170 \\
\hline & 7.1346 & -4.4516 & 0.1895 \\
\hline & 7.0829 & -5.3565 & -1.3290 \\
\hline & 2970 & -4.5769 & -3.3078 \\
\hline & 8.3536 & -2.3075 & $0.32 \mathrm{C}$ \\
\hline & 9.3700 & -2.6812 & -4.5068 \\
\hline & 9.3869 & -0.4023 & -0.8589 \\
\hline & 9.9257 & -0.5898 & -3.2792 \\
\hline & -3.1457 & 0.8344 & 0.4340 \\
\hline & -1.8910 & 1.0012 & -0.0830 \\
\hline & -1.6441 & 2.2333 & -0.7775 \\
\hline & -0.4297 & 2.5778 & -1.1781 \\
\hline & -2.6677 & 3.0996 & -1.0311 \\
\hline & -3.8734 & 2.8320 & -0.5562 \\
\hline & -4.1280 & 1.7470 & 0.2049 \\
\hline & 1.2772 & -1.0846 & -2.9092 \\
\hline & 0.8895 & -0.0833 & -1.5555 \\
\hline & -5.0108 & 3.7357 & -0.8995 \\
\hline & -0.2226 & -0.6187 & -1.0134 \\
\hline & -0.8021 & -1.7712 & -1.5994 \\
\hline & -2.0702 & -2.3540 & -1.0749 \\
\hline & -0.0846 & -2.1742 & -2.6811 \\
\hline & -0.3384 & -3.3264 & -3.5977 \\
\hline & -0.7867 & 0.0237 & 0.1983 \\
\hline & 0.3623 & 1.9332 & -1.1038 \\
\hline & -0.2388 & 3.4951 & -1.5838 \\
\hline & -1.1284 & -0.7575 & 0.8764 \\
\hline & 0.0522 & 0.5416 & 0.6669 \\
\hline & -5.7364 & 3.7708 & -0.0827 \\
\hline & -4.6379 & 4.7285 & -1.1508 \\
\hline & -5.5312 & 3.3244 & -1.7726 \\
\hline & -3.4306 & -0.0163 & 1.0449 \\
\hline & -2.0419 & -2.5035 & 0.0057 \\
\hline & -2.2647 & -3.3190 & -1.5432 \\
\hline $\mathrm{H}$ & -2.9102 & -1.7025 & -1.3190 \\
\hline & 0.5021 & -4.0313 & -3.6004 \\
\hline & -0.4845 & -2.9878 & -4.6297 \\
\hline & 1. 3252 & -2.4273 & 7.6677 \\
\hline & 0.7542 & -1.0050 & 7.6708 \\
\hline & 1.4461 & -0.0770 & 6.6612 \\
\hline & 1.7629 & -0.5887 & 5.5437 \\
\hline & 1.6289 & 1.1291 & \\
\hline 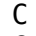 & -8.5948 & 3.1117 & 3.6988 \\
\hline C & -7.8010 & 1.8975 & 3.2239 \\
\hline & -7.0139 & 2.1305 & 1.9307 \\
\hline & -7.1997 & 3.1 & \\
\hline 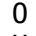 & -6.1983 & 1.1991 & 1.6105 \\
\hline $\mathrm{H}$ & -9.1297 & 2.8905 & 4.6292 \\
\hline & -7.9344 & 3.9628 & 3.8818 \\
\hline & -8.4626 & 1. & 532 \\
\hline 11 & -7.0819 & 1.5678 & 3.9822 \\
\hline & -1.1596 & 2.2672 & 5.3633 \\
\hline & -0.5400 & 3.23 & 4.3456 \\
\hline & 0.7577 & 42 & 7590 \\
\hline & 1.2326 & 2.7758 & 2.4635 \\
\hline & 1.7933 & 2.2809 & 4.5306 \\
\hline & 2.8 & 1.9958 & 3. \\
\hline & 2.5278 & 2.2898 & 2.4431 \\
\hline & -1.3803 & 1.3030 & 4.8928 \\
\hline & -1.2378 & 3.4135 & 3.5194 \\
\hline & -0.38 & 4.2 & 303 \\
\hline & 0.7474 & 3.1046 & 1.5564 \\
\hline & 3.7412 & 1.5437 & 4.0425 \\
\hline & 3.5079 & 1.8402 & 1.2471 \\
\hline & 5.299 & 7.10 & 2.7322 \\
\hline & 5.3355 & 5.86 & 3.6338 \\
\hline & 6.1132 & 4.6695 & 3.0630 \\
\hline & 6.0962 & 3.5140 & 4.0752 \\
\hline & 5.559 & 4.2142 & 1.7084 \\
\hline & 4.7506 & 6.9117 & 1.8055 \\
\hline & 4.3066 & 5.5451 & 3.8443 \\
\hline & 5.7 & 6.1408 & 4.6036 \\
\hline & 7.1580 & 4.9722 & 2.9058 \\
\hline & 5.0738 & 3.3169 & 4.4118 \\
\hline & 6.4820 & 2.586 & 3.6413 \\
\hline & 6.693 & 3.132 & 4.9623 \\
\hline & 015 & 3.9443 & 1.7849 \\
\hline & 6.1098 & 3.3365 & 1.3624 \\
\hline & 560 & 003 & 0.946 \\
\hline
\end{tabular}




\begin{tabular}{|c|c|c|c|}
\hline $\mathrm{H}$ & -1.2307 & -3.8777 & -3.2979 \\
\hline $\mathrm{H}$ & -9.3236 & 3.4181 & 2.9465 \\
\hline $\mathrm{H}$ & 11.9583 & 4.0791 & -0.7153 \\
\hline $\mathrm{H}$ & 11.6443 & 5.7676 & -1.1686 \\
\hline $\mathrm{H}$ & -10.1718 & 2.8265 & -5.1996 \\
\hline $\mathrm{H}$ & -9.1552 & 1.4246 & -5.5781 \\
\hline $\mathrm{H}$ & -0.4831 & 2.0856 & 6.2026 \\
\hline $\mathrm{H}$ & -2.0972 & 2.6734 & 5.7551 \\
\hline $\mathrm{H}$ & 6.3117 & 7.4240 & 2.4578 \\
\hline $\mathrm{H}$ & 4.8089 & 7.9464 & 3.2344 \\
\hline $\mathrm{H}$ & -0.3101 & -1.0320 & 7.3925 \\
\hline $\mathrm{H}$ & 0.8042 & -0.5451 & 8.6618 \\
\hline H & 1.3599 & -2.8150 & 6.6472 \\
\hline H & 0.7291 & -3.1023 & 8.2916 \\
\hline $\mathrm{H}$ & 2.3518 & -2.4342 & 8.0492 \\
\hline $\mathrm{H}$ & 8.4722 & -6.5689 & 0.3671 \\
\hline $\mathrm{H}$ & 9.4735 & -6.0576 & -1.0045 \\
\hline $\mathrm{H}$ & 4.3345 & -6.8641 & -1.6574 \\
\hline $\mathrm{H}$ & 0.4437 & -7.4294 & -4.8195 \\
\hline $\mathrm{H}$ & -1.2801 & -7.6404 & -5.1577 \\
\hline $\mathrm{H}$ & 4.1881 & -3.8195 & -4.9446 \\
\hline $\mathrm{H}$ & 1.7518 & 1.9522 & 5.5288 \\
\hline $\mathrm{H}$ & -5.0963 & 1.5841 & 0.7189 \\
\hline $\mathrm{C}$ & 2.1553 & 6.6241 & -3.1570 \\
\hline $\mathrm{C}$ & 0.8506 & 6.1024 & -2.5954 \\
\hline 0 & 0.7022 & 4.9157 & -2.3064 \\
\hline $\mathrm{N}$ & -0.1433 & 7.0218 & -2.4182 \\
\hline C & -1.5012 & 6.5496 & -2.1987 \\
\hline C & -2.0958 & 6.0618 & -3.5272 \\
\hline 0 & -1.9508 & 6.7325 & -4.5493 \\
\hline $\mathrm{N}$ & -2.7547 & 4.8873 & -3.4669 \\
\hline $\mathrm{C}$ & -3.3124 & 4.2697 & -4.6618 \\
\hline C & -3.3912 & 2.7495 & -4.5124 \\
\hline $\mathrm{C}$ & -2.0419 & 2.0435 & -4.2986 \\
\hline C & -2.2600 & 0.5801 & -3.9054 \\
\hline $\mathrm{C}$ & -1.1229 & 2.1542 & -5.5205 \\
\hline $\mathrm{C}$ & 3.5819 & 3.0350 & -2.5529 \\
\hline $\mathrm{C}$ & 4.7284 & 3.7470 & -2.9276 \\
\hline $\mathrm{C}$ & 4.8509 & 4.2408 & -4.2259 \\
\hline$C$ & 3.8403 & 4.0052 & -5.1601 \\
\hline C & 2.6950 & 3.2970 & -4.7878 \\
\hline $\mathrm{C}$ & 2.5584 & 2.8270 & -3.4852 \\
\hline $\mathrm{C}$ & 3.4021 & 2.4968 & -1.1717 \\
\hline 0 & 2.3458 & 2.6093 & -0.5801 \\
\hline $\mathrm{H}$ & 2.1959 & 7.7147 & -3.2040 \\
\hline $\mathrm{H}$ & 2.9775 & 6.2486 & -2.5450 \\
\hline $\mathrm{H}$ & -1.4874 & 5.7678 & -1.4386 \\
\hline $\mathrm{H}$ & -2.1047 & 7.3831 & -1.8281 \\
\hline $\mathrm{H}$ & -4.0583 & 2.5067 & -3.6767 \\
\hline $\mathrm{H}$ & -3.8789 & 2.3410 & -5.4072 \\
\hline $\mathrm{H}$ & -1.5289 & 2.5362 & -3.4677 \\
\hline $\mathrm{H}$ & -0.1802 & 1.6271 & -5.3376 \\
\hline $\mathrm{H}$ & -1.5895 & 1.7055 & -6.4066 \\
\hline $\mathrm{H}$ & -0.8829 & 3.1959 & -5.7560 \\
\hline $\mathrm{H}$ & -2.7516 & 0.0245 & -4.7135 \\
\hline $\mathrm{H}$ & -2.8964 & 0.4924 & -3.0197 \\
\hline $\mathrm{H}$ & -1.3099 & 0.0863 & -3.6900 \\
\hline $\mathrm{H}$ & -2.6889 & 4.5641 & -5.5108 \\
\hline $\mathrm{H}$ & 5.5160 & 3.9312 & -2.2033 \\
\hline $\mathrm{H}$ & 5.7357 & 4.8031 & -4.5083 \\
\hline $\mathrm{H}$ & 3.9433 & 4.3763 & -6.1757 \\
\hline $\mathrm{H}$ & 1.9036 & 3.1188 & -5.5090 \\
\hline $\mathrm{H}$ & 1.6739 & 2.2810 & -3.1808 \\
\hline $\mathrm{C}$ & -5.4260 & -2.9231 & 3.6197 \\
\hline $\mathrm{C}$ & -4.0226 & -2.3350 & 3.8537 \\
\hline 0 & -3.8636 & -1.1265 & 4.0063 \\
\hline$C$ & -6.2833 & -2.0334 & 2.7014 \\
\hline C & -5.8833 & -2.1294 & 1.2450 \\
\hline $\mathrm{N}$ & -6.7315 & -2.7655 & 0.4054 \\
\hline 0 & -4.8129 & -1.6529 & 0.8291 \\
\hline $\mathrm{N}$ & -2.9931 & -3.2167 & 3.9937 \\
\hline $\mathrm{C}$ & -1.6621 & -2.7494 & 4.3755 \\
\hline C & -0.8829 & -2.1869 & 3.1820 \\
\hline 0 & -1.1722 & -2.5228 & 2.0252 \\
\hline C & -0.9633 & -4.0465 & 4.8342 \\
\hline $\mathrm{C}$ & -1.5443 & -5.0891 & 3.8660 \\
\hline $\mathrm{C}$ & -3.0074 & -4.6503 & 3.6746 \\
\hline $\mathrm{N}$ & 0.1571 & -1.4044 & 3.5105 \\
\hline $\mathrm{C}$ & 1.2385 & -1.1223 & 2.5959 \\
\hline C & 2.5171 & -1.8380 & 3.0436 \\
\hline & & & \\
\hline
\end{tabular}

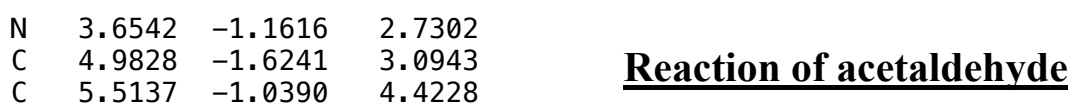

$\begin{array}{llll}0 & 6.6996 & -1.1771 & 4.7171\end{array}$

C $\quad 6.0117-1.3296 \quad 1.9941$

$\begin{array}{llll}0 & 6.2822 & 0.0584 & 1.8609\end{array}$

N $\quad 4.6264 \quad-0.3636 \quad 5.1878$

$\begin{array}{llll}\mathrm{C} & 5.0602 & 0.2370 & 6.4367\end{array}$

$\begin{array}{llll}\text { H } & -7.3374 & -2.3031 & 2.8112\end{array}$

H $\quad-6.1451 \quad-0.9843 \quad 2.9789$

H $\quad-5.3910-3.9473 \quad 3.2406$

H $\quad-1.2433 \quad-4.2672 \quad 5.8694$

$\mathrm{H} \quad 0.1242-3.9652 \quad 4.7716$

$\mathrm{H} \quad-1.4670 \quad-6.1134 \quad 4.2391$

$\mathrm{H} \quad-1.0194 \quad-5.0270 \quad 2.9100$

$\mathrm{H} \quad-3.6808 \quad-5.1919 \quad 4.3517$

H $\quad-3.3438 \quad-4.8183 \quad 2.6460$

$\mathrm{H} \quad-1.7410-1.9954 \quad 5.1617$

H $\quad 1.4050 \quad-0.0456 \quad 2.5194$

H $\quad 0.9657-1.5061 \quad 1.6114$

H $6.9487 \quad-1.8033 \quad 2.2905$

H $\quad 5.6685 \quad-1.7589 \quad 1.0438$

H $\quad 5.4961 \quad 0.5205 \quad 1.4800$

$\begin{array}{llll}\mathrm{H} & 4.9074 & -2.7084 & 3.2268\end{array}$

H $\quad 5.9011 \quad 0.9110 \quad 6.2524$

$\begin{array}{lll}2.2905 & 6.2075 & -4.1585\end{array}$

$\begin{array}{lll}-4.3157 & 4.6715 & -4.8584\end{array}$

$\begin{array}{lll}-2.7147 & 4.3402 & -2.6050\end{array}$

$\begin{array}{lll}-0.0720 & 7.8682 & -2.9659\end{array}$

$\begin{array}{lll}5.3918 & -0.5184 & 7.1599\end{array}$

$\begin{array}{lll}4.2206 & 0.7946 & 6.8548\end{array}$

$3.6184-0.4835 \quad 5.0674$

$0.4287-1.2408 \quad 4.4872$

$\begin{array}{lll}-7.6440 & -3.1133 & 0.7002\end{array}$

$\begin{array}{lll}-6.5198 & -2.7203 & -0.5859\end{array}$

$\begin{array}{lll}3.5646 & -0.2524 & 2.2951\end{array}$

H $\quad-5.9025 \quad-2.9671 \quad 4.6061$

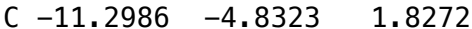

C $-10.2878-4.2336 \quad 0.8720$

$\begin{array}{llll}0 & -9.1257 & -4.0232 & 1.2333\end{array}$

$\begin{array}{llll}C & -11.5978 & -3.8046 & 2.9237\end{array}$

N $-10.7477-3.8822-0.3483$

C $\quad-9.9140-3.1832-1.3100$

$\begin{array}{llll}\text { C } & -9.0464 & 7.3068 & 2.5657\end{array}$

$\begin{array}{llll}\text { C } & -8.9969 & 6.0267 & 1.7385\end{array}$

$\begin{array}{llll}C & -8.8069 & 6.2721 & 0.2433\end{array}$

$\mathrm{N} \quad-8.3680 \quad 5.1739 \quad-0.4369$

$\begin{array}{llll}0 & -9.0641 & 7.3424 & -0.3000\end{array}$

$\mathrm{H}-10.6707-3.5133 \quad 3.4223$

H $-12.2819 \quad-4.2147 \quad 3.6718$

H $-12.0549-2.9036 \quad 2.5028$

H $-12.2164-5.1315 \quad 1.3087$

H $\quad-9.5500 \quad-2.2354-0.9014$

$\begin{array}{lrrr}H & -8.2092 & 5.3521 & 2.0808\end{array}$

$\mathrm{H} \quad-9.9355 \quad 5.4678 \quad 1.8534$

$\begin{array}{llll}\mathrm{H} & -7.9931 & 4.3721 & 0.0667\end{array}$

$\mathrm{H} \quad-8.1425 \quad 5.3001-1.4120$

H $\quad-9.8078 \quad 7.9872 \quad 2.1764$

$\begin{array}{llll}H & -9.2706 & 7.0828 & 3.6132\end{array}$

$\begin{array}{llll}\mathrm{H} & -8.0888 & 7.8353 & 2.5275\end{array}$

H $-10.8524-5.7308 \quad 2.2633$

$\begin{array}{lll}\mathrm{H}-10.4999 & -2.9782 & -2.2064\end{array}$

H $\quad-9.0459-3.7900 \quad-1.5874$

$\mathrm{H}-11.7118-4.0660-0.5751$

$\begin{array}{llll}\text { C } & 5.7035 & -1.6011 & -3.0199\end{array}$

C $\quad 4.7197 \quad-1.7448-2.0441$

$\begin{array}{llll}\text { C } & 4.3590 & -0.6645 & -1.2395\end{array}$

$4.9890 \quad 0.5714-1.4011$

$5.9711 \quad 0.7127-2.3862$

$6.3240-0.3648-3.1947$

$6.0064-2.4505-3.6198$

$\begin{array}{lll}4.2302 & -2.7014 & -1.9114\end{array}$

$3.5925-0.7636-0.4824$

$6.4586 \quad 1.6715 \quad-2.5250$

$7.1022-0.2496-3.9404$

$4.5751 \quad 1.7448 \quad-0.5243$

$\begin{array}{lll}4.2177 & 1.2859 & 0.7499\end{array}$

$\begin{array}{lll}7.6888 & 0.9799 & 1.1991\end{array}$

$\begin{array}{lll}5.4228 & 2.4468 & -0.4668\end{array}$
En(AA)-S1

\begin{tabular}{|c|c|c|c|}
\hline & 11.2960 & 5.2781 & -0.4878 \\
\hline & 9.8754 & 4.8194 & \\
\hline & 9.2983 & 3.7940 & 0.0845 \\
\hline & 8.6502 & 2.6184 & -0.2273 \\
\hline & 2964 & 9764 & 1.4568 \\
\hline & 6512 & 9335 & 1.9503 \\
\hline & 8.2410 & 2.0743 & 0.9762 \\
\hline & 11.3156 & 5.6512 & 0.5393 \\
\hline & 553 & 185 & -1 . \\
\hline & 9.2171 & 9995 & \\
\hline & 8.4427 & 2.1331 & -1.1675 \\
\hline & 8.4527 & 2.7463 & 2.9958 \\
\hline & 7.5735 & 1.3030 & 1.0968 \\
\hline & -9.4942 & 2.0282 & -4.7089 \\
\hline & -8.2781 & 2.4806 & -3.8830 \\
\hline & -7.4440 & 1.3335 & \\
\hline & -6.6731 & 0.5433 & -4.2174 \\
\hline & -7.4109 & 1.0257 & -1.9872 \\
\hline & -5.9034 & -0.5154 & -3.7429 \\
\hline & -6.6398 & -0 . & -1.4930 \\
\hline & -5.8828 & -0.8080 & -2.3734 \\
\hline & -5.1244 & -1.8665 & -1.9591 \\
\hline & -10.1624 & 1.4084 & -4.1022 \\
\hline & -8.6237 & 3.0913 & $-3.04 e$ \\
\hline & -7.6528 & 3.1362 & -4.5038 \\
\hline & -6.6637 & 0.7682 & -5.2817 \\
\hline & -7.9649 & 1.6248 & -1.2701 \\
\hline & -5.2971 & -1 . & -4 \\
\hline & -6.6131 & -0.2055 & -0.4244 \\
\hline & -4.8897 & -1.7 & -1.0011 \\
\hline & -0.3123 & -6 . & -4 \\
\hline & -0.4153 & -7.3 & -3.4177 \\
\hline & -1.3903 & -6.6087 & -2.543 \\
\hline & -1.0010 & -5.4421 & -1.876 \\
\hline & -2.7090 & & \\
\hline & -1.8723 & -4.7 & -1.0466 \\
\hline & -3.6005 & -6.3512 & -1.5515 \\
\hline & -3.1841 & -5.19 & -0.8852 \\
\hline & -4.0867 & -4 & -0 . \\
\hline & 0.0087 & -5.7 & -4.8 \\
\hline & -0.7068 & -8.4250 & -3.4516 \\
\hline & 0.5801 & -7.3412 & -2.9563 \\
\hline & 0.0142 & -5 & \\
\hline & -3.0426 & -7.5 & -2 \\
\hline & -1.5326 & -3.8655 & -0.4993 \\
\hline & -4.6205 & -6 . & -1.411 \\
\hline & -3 & -3 & \\
\hline & 4.9273 & -4.4485 & -5.2461 \\
\hline & 5.1845 & -4.9100 & -3.8319 \\
\hline & 6.3199 & -5.1 & -3.396 \\
\hline & 6.2057 & $-4 . e$ & -5.9938 \\
\hline & 4.4010 & -5.2553 & -5.7741 \\
\hline & 6.9054 & -4.9168 & -5.9941 \\
\hline & 6.7091 & -3.23 & -5.51 \\
\hline & & -3 & -7 . \\
\hline & 4.0750 & -5.0978 & -3.0819 \\
\hline & 4.1942 & -5.6373 & -1.7450 \\
\hline & 2.9066 & -5 . & -0.9 \\
\hline & 2.3001 & -4.1 & -0.7 \\
\hline & 1.2418 & -4.2775 & 0.38 \\
\hline & 3.3563 & -3.13 & -0.3941 \\
\hline & 3.1622 & -4 & -3 \\
\hline & 4.9752 & -5.0 & -1.2256 \\
\hline & 3.1383 & -6.0239 & 0.0419 \\
\hline & 2.1493 & -6.24 & -1.3866 \\
\hline & & & -1. \\
\hline & 1.7223 & -4.2998 & 1.366 \\
\hline & 0.5671 & -3.4217 & 0.3642 \\
\hline & 0.6281 & -5.1767 & 0.2799 \\
\hline & & & \\
\hline & 3.8827 & -3.3883 & 0.532 \\
\hline & 2.9016 & -2.1517 & -0.2668 \\
\hline & 9.0991 & -5.4207 & -0.387 \\
\hline & & & -0.585 \\
\hline & 8.5499 & -3.0574 & \\
\hline
\end{tabular}




\begin{tabular}{|c|c|c|c|}
\hline C & 8.7436 & -2.8689 & -2.4868 \\
\hline C & 8.9226 & -2.0267 & -0.2380 \\
\hline C & 9.2927 & -1.6814 & -2.9725 \\
\hline C & 9.4672 & -0.8350 & -0.7184 \\
\hline C & 9.6545 & -0.6593 & -2.0911 \\
\hline $\mathrm{H}$ & 9.8570 & -5.0737 & 0.3227 \\
\hline $\mathrm{H}$ & 7.4891 & -4.1860 & 0.3693 \\
\hline 1 & 7.2598 & -4.7526 & -1.2913 \\
\hline $\mathrm{H}$ & 8.4353 & -3.6539 & -3.1718 \\
\hline $\mathrm{H}$ & 8.7788 & -2.1577 & 0.8302 \\
\hline $\mathrm{H}$ & 9.4304 & -1.5499 & -4.0424 \\
\hline $\mathrm{H}$ & 9.7339 & -0.0429 & -0.0269 \\
\hline గ & 10.0760 & 0.2685 & -2.4665 \\
\hline C & -3.1425 & 0.9190 & 0.3655 \\
\hline C & -1.9554 & 1.3019 & -0.2002 \\
\hline C & -1.9722 & 2.5625 & -0.8941 \\
\hline $\mathrm{N}$ & -0.8720 & 3.1156 & -1.3653 \\
\hline $\mathrm{N}$ & -3.1373 & 3.2422 & -1.0906 \\
\hline C & -4.2500 & 2.7835 & -0.5497 \\
\hline $\mathrm{N}$ & -4.2759 & 1.6646 & 0.2047 \\
\hline S & 1.7242 & -0.9375 & -2.9388 \\
\hline C & 1.2301 & 0.2995 & -1.7516 \\
\hline $\mathrm{C}$ & -5.5178 & 3.5332 & -0.7943 \\
\hline $\mathrm{N}$ & -0.1033 & 0.0329 & -1.3527 \\
\hline C & -0.6956 & -1.0537 & -2.0363 \\
\hline C & -2.1384 & -1.3636 & -1.8118 \\
\hline C & 0.1132 & -1.6743 & -2.9166 \\
\hline C & -0.1812 & -2.7547 & -3.9040 \\
\hline C & -0.6947 & 0.4704 & -0.0636 \\
\hline $\mathrm{H}$ & 0.0632 & 2.7790 & -1.1047 \\
\hline $\mathrm{H}$ & -0.8819 & 4.0094 & -1.8436 \\
\hline $\mathrm{H}$ & -0.9184 & -0.4169 & 0.5327 \\
\hline $\mathrm{H}$ & 0.0628 & 1.0418 & 0.4629 \\
\hline $\mathrm{H}$ & -6.3191 & 3.2144 & -0.1261 \\
\hline $\mathrm{H}$ & -5.3328 & 4.6044 & -0.6893 \\
\hline $\mathrm{H}$ & -5.8369 & 3.3458 & -1.8249 \\
\hline $\mathrm{H}$ & -3.2629 & 0.0101 & 0.9484 \\
\hline $\mathrm{H}$ & -2.3524 & -1.5660 & -0.7597 \\
\hline $\mathrm{H}$ & -2.4372 & -2.2401 & -2.3825 \\
\hline $\mathrm{H}$ & -2.7738 & -0.5329 & -2.1293 \\
\hline $\mathrm{H}$ & 0.5929 & -3.5327 & -3.8797 \\
\hline $\mathrm{H}$ & -0.2168 & -2.3654 & -4.9296 \\
\hline C & 1.5177 & -2.5248 & 7.5576 \\
\hline C & 0.6871 & -1.2609 & 7. 3138 \\
\hline C & 1.4865 & -0.2434 & 6.4968 \\
\hline 0 & 1.7379 & -0.5898 & 5.2695 \\
\hline 0 & 1.8651 & 0.8136 & 7.0199 \\
\hline C & -8.5430 & 2.8886 & 3.7746 \\
\hline C & -7.8722 & 1.5983 & 3.3018 \\
\hline C & -7.2920 & 1.6936 & 1.8841 \\
\hline 0 & -7.8227 & 2.4894 & 1.0707 \\
\hline 0 & -6.3042 & 0.9246 & 1.6358 \\
\hline $\mathrm{H}$ & -8.9881 & 2.7616 & 4.7675 \\
\hline $\mathrm{H}$ & -7.8204 & 3.7072 & 3.8357 \\
\hline $\mathrm{H}$ & -8.6010 & 0.7753 & 3.2978 \\
\hline $\mathrm{H}$ & -7.0666 & 1.2903 & 3.9747 \\
\hline C & -1.0829 & 2.1701 & 5.3855 \\
\hline C & -0.9024 & 1.6288 & 3.9643 \\
\hline C & 0.3876 & 2.0406 & 3.3349 \\
\hline C & 0.6509 & 2.8020 & 2.2205 \\
\hline $\mathrm{N}$ & 1.6258 & 1.6449 & 3.8014 \\
\hline C & 2.5638 & 2.1306 & 2.9553 \\
\hline $\mathrm{N}$ & 2.0135 & 2.8401 & 1.9735 \\
\hline $\mathrm{H}$ & -0.2632 & 1.8573 & 6.0384 \\
\hline $\mathrm{H}$ & -0.9721 & 0.5363 & 3.9727 \\
\hline $\mathrm{H}$ & -1.7166 & 1.9754 & 3. 3186 \\
\hline $\mathrm{H}$ & -0.0524 & 3.2998 & 1.5685 \\
\hline $\mathrm{H}$ & 3.6213 & 1.9648 & 3.0935 \\
\hline $\mathrm{H}$ & 1.9816 & 2.4869 & 0.3111 \\
\hline C & 5.2525 & 7.2194 & 2.8540 \\
\hline C & 5.1503 & 5.9892 & 3.7618 \\
\hline C & 5.8647 & 4.7278 & 3.2409 \\
\hline C & 5.7602 & 3.6031 & 4. 2818 \\
\hline C & 5.3149 & 4.2699 & 1.8829 \\
\hline $\mathrm{H}$ & 4.7595 & 7.0505 & 1.8916 \\
\hline $\mathrm{H}$ & 4.0913 & 5.7452 & 3.9253 \\
\hline $\mathrm{H}$ & 5.5610 & 6.2386 & 4.7495 \\
\hline $\mathrm{H}$ & 6.9282 & 4.9688 & 3.1040 \\
\hline $\mathrm{H}$ & 4.7126 & 3.3784 & 4.5119 \\
\hline $\mathrm{H}$ & 6.2278 & 2.6763 & 3.9321 \\
\hline$\pi$ & 6.2436 & 3.8873 & 5.2234 \\
\hline
\end{tabular}

\begin{tabular}{|c|c|c|c|}
\hline $\mathrm{H}$ & 4.2248 & 4.1667 & 1.9093 \\
\hline & 5.7410 & 3.3006 & 1.6060 \\
\hline & 5.5655 & 4.9771 & 1.0859 \\
\hline $\mathrm{H}$ & -1.1313 & -3.2434 & -3.6852 \\
\hline $\mathrm{H}$ & -9.3247 & 3.1840 & 3.0747 \\
\hline $\mathrm{H}$ & 12.0049 & 4.4459 & -0.5520 \\
\hline 11 & 11.6366 & 6.0738 & -1.1588 \\
\hline $\mathrm{H}$ & -10.0641 & 2.8866 & -5.0798 \\
\hline $\mathrm{H}$ & -9.1800 & 1.4304 & -5.5706 \\
\hline $\mathrm{H}$ & -1.1048 & 3.2649 & 5.3828 \\
\hline $\mathrm{H}$ & -2.0234 & 1.8094 & 5.8135 \\
\hline $\mathrm{H}$ & 6.2995 & 7.4720 & 2.6496 \\
\hline $\mathrm{H}$ & 4.7811 & 8.0923 & 3.3182 \\
\hline $\mathrm{H}$ & -0.2252 & -1.5201 & 6.7610 \\
\hline $\mathrm{H}$ & 0.3930 & -0.7963 & 8.2586 \\
\hline $\mathrm{H}$ & 1.8303 & -2.9584 & 6.6030 \\
\hline $\mathrm{H}$ & 0.9533 & -3.2791 & 8.1166 \\
\hline $\mathrm{H}$ & 2.4211 & -2.2875 & 8.1295 \\
\hline $\mathrm{H}$ & 8.6769 & -6.3573 & -0.0088 \\
\hline $\mathrm{H}$ & 9.6014 & -5.6334 & -1.3362 \\
\hline $\mathrm{H}$ & 4.5557 & -6.6731 & -1.8042 \\
\hline $\mathrm{H}$ & 0.4054 & -7.4099 & -5.4457 \\
\hline $\mathrm{H}$ & -1.2842 & -6.8737 & -5.3566 \\
\hline $\mathrm{H}$ & 4.2310 & -3.6006 & -5.2239 \\
\hline $\mathrm{H}$ & 1.7854 & 0.9057 & 4.5276 \\
\hline $\mathrm{H}$ & -5.1892 & 1.3603 & 0.7165 \\
\hline C & 2.0928 & 6.8193 & -3.0326 \\
\hline C & 0.7194 & 6.3539 & -2.6170 \\
\hline 0 & 0.4617 & 5.1577 & -2.5007 \\
\hline $\mathrm{N}$ & -0.2210 & 7.3122 & -2.3735 \\
\hline C & -1.6086 & 6.8919 & -2.2654 \\
\hline C & -2.0743 & 6.2368 & -3.5783 \\
\hline 0 & -1.7192 & 6.7140 & -4.6550 \\
\hline $\mathrm{N}$ & -2.8822 & 5.1648 & -3.4321 \\
\hline C & -3.3278 & 4.3812 & -4.5750 \\
\hline $\mathrm{C}$ & -2.6486 & 3.0069 & -4.6575 \\
\hline C & -1.1229 & 3.0268 & -4.8491 \\
\hline C & -0.5635 & 1.6050 & -4.7273 \\
\hline C & -0.7141 & 3.6816 & -6.1740 \\
\hline C & 3.3760 & 1.6598 & -1.9141 \\
\hline $\mathrm{C}$ & 4.2718 & 2.3605 & -1.0792 \\
\hline C & 5.5352 & 2.7315 & -1.5224 \\
\hline $\mathrm{C}$ & 5.9461 & 2.4212 & -2.8211 \\
\hline C & 5.0554 & 1.7669 & -3.6753 \\
\hline C & 3.7875 & 1.3972 & -3.2330 \\
\hline$C$ & 2.0403 & 1.3372 & -1.3891 \\
\hline 0 & 1.5120 & 2.3036 & -0.5519 \\
\hline $\mathrm{H}$ & 2.2836 & 7.8668 & -2.7857 \\
\hline $\mathrm{H}$ & 2.8364 & 6.1831 & -2.5499 \\
\hline $\mathrm{H}$ & -1.7211 & 6.2177 & -1.4123 \\
\hline $\mathrm{H}$ & -2.2205 & 7.7777 & -2.0755 \\
\hline $\mathrm{H}$ & -2.8901 & 2.4461 & -3.7463 \\
\hline $\mathrm{H}$ & -3.1117 & 2.4526 & -5.4864 \\
\hline $\mathrm{H}$ & -0.6812 & 3.6218 & -4.0438 \\
\hline $\mathrm{H}$ & 0.3723 & 3.6305 & -6.3056 \\
\hline $\mathrm{H}$ & -1.1763 & 3.1682 & -7.0276 \\
\hline $\mathrm{H}$ & -1.0007 & 4.7363 & -6.2028 \\
\hline $\mathrm{H}$ & -0.9562 & 0.9547 & -5.5197 \\
\hline $\mathrm{H}$ & -0.8259 & 1.1580 & -3.7650 \\
\hline $\mathrm{H}$ & 0.5283 & 1.6020 & -4.8018 \\
\hline $\mathrm{H}$ & -3.1296 & 4.9799 & -5.4650 \\
\hline $\mathrm{H}$ & 3.9829 & 2.6149 & -0.0661 \\
\hline $\mathrm{H}$ & 6.2035 & 3.2573 & -0.8504 \\
\hline $\mathrm{H}$ & 6.9330 & 2.7121 & -3.1690 \\
\hline $\mathrm{H}$ & 5.3412 & 1.5577 & -4.7028 \\
\hline $\mathrm{H}$ & 3.1057 & 0.9228 & -3.9275 \\
\hline C & -5.2392 & -3.0688 & 3.5258 \\
\hline C & -3.8191 & -2.4892 & 3.6341 \\
\hline 0 & -3.6436 & -1.2928 & 3.8615 \\
\hline$C$ & -6.1278 & -2.1941 & 2.6199 \\
\hline $\mathrm{C}$ & -5.7688 & -2.3628 & 1.1622 \\
\hline $\mathrm{N}$ & -6.6589 & -2.9694 & 0.3707 \\
\hline 0 & -4.6669 & -1.9577 & 0.7110 \\
\hline $\mathrm{N}$ & -2.7763 & -3.3588 & 3.5522 \\
\hline C & -1.4219 & -2.9046 & 3.8478 \\
\hline C & -0.7522 & -2.1889 & 2.6663 \\
\hline 0 & -1.1486 & -2.3286 & 1.5021 \\
\hline C & -0.6639 & -4.2241 & 4.1134 \\
\hline C & -1.3492 & -5.1943 & 3.1401 \\
\hline C & -2.8292 & -4.7896 & 3.2081 \\
\hline $\mathrm{N}$ & 7 & 77 & \\
\hline
\end{tabular}

\begin{tabular}{|c|c|c|c|}
\hline C & 1.4070 & -1.1413 & 2 \\
\hline C & 2.7298 & -1.735 & 2.601 \\
\hline U & 7999 & -2.87 & \\
\hline & 3.7999 & -0.90 & \\
\hline$C$ & 5.1464 & -1.279 & \\
\hline C & 5.6570 & -0.5 & \\
\hline 0 & 6.8645 & -0.34 & \\
\hline C & 325 & -1.0 & \\
\hline 0 & 6.0259 & 0.26 & \\
\hline & 4.7408 & -0.24 & \\
\hline C & 5.1862 & 0.25 & \\
\hline $\mathrm{H}$ & -7.1804 & -2.44 & 2.7 \\
\hline$\Pi$ & -5.9767 & -1.13 & 2. \\
\hline$\pi$ & -5. & -4.10 & \\
\hline$\Pi$ & -0.8281 & -4.532 & \\
\hline 11 & 0.4 & -4 & \\
\hline $\mathrm{H}$ & -1.1973 & -6. & 3. \\
\hline & -0.9 & -5 . & \\
\hline $\mathrm{H}$ & -3.3 & -5 & 3. \\
\hline$\Pi$ & -3.3 & -4.947 & 2. \\
\hline 11 & -1.4 & -2.2 & 4. \\
\hline H & 1.4860 & -0.05 & 1. \\
\hline & 1.1 & -1.56 & \\
\hline $\mathrm{H}$ & 7.1383 & -1.20 & 2.13 \\
\hline H & 5. & -1.78 & 0. \\
\hline H & 5.6 & & \\
\hline $\mathrm{H}$ & 5.1060 & -2.34 & 3.1 \\
\hline & 5. & & \\
\hline H & 2.1891 & 6. & \\
\hline $\mathrm{H}$ & -4.4 & 4.2406 & -4 \\
\hline H & -3 & 4. & -2 . \\
\hline $\mathrm{H}$ & -0. & 8.2258 & -2.7 \\
\hline $\mathrm{H}$ & 5. & -0. & \\
\hline $\mathrm{H}$ & 4.3097 & 0. & 6. \\
\hline H & 3.7 & -0. & 4. \\
\hline H & 0.6 & -1 & \\
\hline H & -7.5 & -3.4 & 0.7 \\
\hline $\mathrm{H}$ & -6.3 & -3 & -0.5 \\
\hline H & 3.6828 & 0. & 2. \\
\hline H & -5.6511 & -3.05 & 4. \\
\hline C & -11.2 & -5 & \\
\hline C & -10.1894 & -4.4729 & 0.71 \\
\hline 0 & -9. & -4 . & 1. \\
\hline C & -11.3 & -4 & 2. \\
\hline 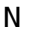 & -10.6 & -3.98 & -0.4 \\
\hline C & -9.7 & -3 & -1.3 \\
\hline C & $-9 . l$ & 7. & 2.75 \\
\hline C & -9. & 5. & \\
\hline C & -9.0438 & 6. & 0.4 \\
\hline $\mathrm{N}$ & -8 & 4. & -0.2 \\
\hline 0 & -9 & 7 & -0 . \\
\hline $\mathrm{H}$ & -10.4273 & -3 & 3. \\
\hline $\mathrm{H}$ & -12. & -4 . & 3. \\
\hline $\mathrm{H}$ & -11.7485 & -3 & 2. \\
\hline $\mathrm{H}$ & -12.2008 & -5.1792 & 1. \\
\hline $\mathrm{H}$ & -9.3 & -2 . & -0 . \\
\hline $\mathrm{H}$ & -8.1851 & 967 & 2. \\
\hline $\mathrm{H}$ & -9. & 5. & 2. \\
\hline $\mathrm{H}$ & -8.5146 & 3. & 0. \\
\hline $\mathrm{H}$ & -8 & 4. & -1.2 \\
\hline $\mathrm{H}$ & -9 & & \\
\hline $\mathrm{H}$ & -9.1568 & 6. & 3. \\
\hline $\mathrm{H}$ & -8.1 & & 2. \\
\hline $\mathrm{H}$ & -10.8952 & -6 & 1.99 \\
\hline $\mathrm{H}$ & -10.3094 & -3 & -2.2 \\
\hline $\mathrm{H}$ & -8.9285 & -3 & -1 . \\
\hline $\mathrm{H}$ & -11.6169 & -4 & -0 . \\
\hline C & 5.9679 & -0 . & -2.03 \\
\hline C & 5.6775 & -1.5337 & -3.29 \\
\hline $\mathrm{H}$ & 6.1383 & -2 . & -3 . \\
\hline $\mathrm{H}$ & 4.6000 & -1 . & -3 \\
\hline $\mathrm{H}$ & & -1 & -4.15 \\
\hline 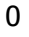 & 5.1451 & -0 . & -1.14 \\
\hline & 216 & -0 & \\
\hline
\end{tabular}

\section{En(AA)-S2}

$$
\begin{array}{rrrr}
\text { C } & 11.2987 & 5.2653 & -1.0770 \\
\text { C } & 9.8400 & 4.7904 & -1.1729 \\
\text { C } & 9.3848 & 3.9413 & -0.0192
\end{array}
$$




\begin{tabular}{|c|c|c|c|c|c|c|c|c|c|c|c|}
\hline C & 8.6783 & 2.7585 & -0.0510 & $\mathrm{H}$ & 8.7893 & -2.3657 & 0.7678 & $\mathrm{H}$ & -0.9746 & 3.5756 & 5.1178 \\
\hline $\mathrm{N}$ & 9.5826 & 4.3291 & 1.2951 & $\mathrm{H}$ & 8.3617 & -1.5009 & -4.0883 & $\mathrm{H}$ & -1.8578 & 2.1688 & 5.7418 \\
\hline C & 8.9985 & 3.4003 & 2.0317 & $\mathrm{H}$ & 9.3545 & -0.1312 & -0.1336 & $\mathrm{H}$ & 6.3759 & 7.6155 & 2.0502 \\
\hline $\mathrm{N}$ & 8.4382 & 2.4216 & 1.2676 & $\mathrm{H}$ & 9.1441 & 0.3125 & -2.5689 & $\mathrm{H}$ & 4.8833 & 8.2922 & 2.7213 \\
\hline $\mathrm{H}$ & 11.4556 & 5.7953 & -0.1341 & C & -2.7808 & 1.0009 & 0.4415 & $\mathrm{H}$ & -0.0154 & -1.0925 & 6.8363 \\
\hline $\mathrm{H}$ & 9.6829 & 4.2341 & -2.1053 & C & -1.6366 & 1.2496 & -0.2716 & $\mathrm{H}$ & 0.6537 & -0.2657 & 8.2560 \\
\hline $\mathrm{H}$ & 9.1911 & 5.6756 & -1.2340 & C & -1.6718 & 2.4212 & -1.1032 & $\mathrm{H}$ & 2.0184 & -2.5632 & 6.7251 \\
\hline $\mathrm{H}$ & 8.3257 & 2.1449 & -0.8637 & $\mathrm{~N}$ & -0.6089 & 2.8744 & -1.7371 & $\mathrm{H}$ & 1.1853 & -2.7620 & 8.2834 \\
\hline $\mathrm{H}$ & 8.9495 & 3.3824 & 3.1110 & $\mathrm{~N}$ & -2.8243 & 3.1215 & -1.2763 & $\mathrm{H}$ & 2.6628 & -1.7913 & 8.1794 \\
\hline $\mathrm{H}$ & 7.8002 & 1.6891 & 1.5765 & C & -3.9002 & 2.7932 & -0.5900 & $\mathrm{H}$ & 8.8625 & -6.3908 & 0.1259 \\
\hline C & -9.5729 & 1.7210 & -4.6021 & $\mathrm{~N}$ & -3.8910 & 1.7851 & 0.3117 & $\mathrm{H}$ & 9.7284 & -5.5753 & -1.1886 \\
\hline C & -8.8239 & 2.5818 & -3.5728 & $\mathrm{~S}$ & 1.6978 & -1.0624 & -3.2743 & $\mathrm{H}$ & 5.0511 & -6.4107 & -1.4149 \\
\hline C & -7.7113 & 1.7970 & -2.9232 & C & 1.3871 & 0.1292 & -1.9887 & $\mathrm{H}$ & 0.2309 & -7.8967 & -4.9041 \\
\hline C & -6.5396 & 1.5127 & -3.6407 & $\mathrm{C}$ & -5.1457 & 3.5707 & -0.8293 & $\mathrm{H}$ & -0.7609 & -6.4573 & -5.2135 \\
\hline C & -7.8398 & 1.2609 & -1.6377 & $\mathrm{~N}$ & 0.1151 & -0.1447 & -1.4413 & $\mathrm{H}$ & 3.8899 & -5.1125 & -5.4588 \\
\hline C & -5.5434 & 0.7086 & -3.1050 & C & -0.5042 & -1.3022 & -1.9674 & $\mathrm{H}$ & 1.9755 & 1. 1265 & 4.4145 \\
\hline C & -6.8487 & 0.4522 & -1.0789 & C & -1.8275 & -1.7372 & -1.4426 & $\mathrm{H}$ & -4.7720 & 1.5736 & 0.9384 \\
\hline C & -5.6987 & 0.1666 & -1.8220 & $\mathrm{C}$ & 0.1914 & -1.9175 & -2.9420 & C & 2.0329 & 6.6298 & -3.4924 \\
\hline 0 & -4.6847 & -0.6041 & -1.3692 & C & -0.1081 & -3.1673 & -3.7027 & C & 0.6695 & 6.1279 & -3.1025 \\
\hline $\mathrm{H}$ & -10.0061 & 0.8382 & -4.1206 & C & -0.3917 & 0.3903 & -0.1543 & 0 & 0.4110 & 4.9257 & -3.1397 \\
\hline $\mathrm{H}$ & -9.5234 & 2.9420 & -2.8095 & $\mathrm{H}$ & 0.3363 & 2.5728 & -1.4736 & $\mathrm{~N}$ & -0.2503 & 7.0435 & -2.7003 \\
\hline $\mathrm{H}$ & -8.4189 & 3.4709 & -4.0725 & $\mathrm{H}$ & -0.6509 & 3.7276 & -2.2873 & C & -1.6292 & 6.6162 & -2.5331 \\
\hline $\mathrm{H}$ & -6.4083 & 1.9281 & -4.6371 & $\mathrm{H}$ & -0.6004 & -0.4520 & 0.5078 & C & -2.1659 & 5.9912 & -3.8347 \\
\hline $\mathrm{H}$ & -8.7229 & 1.4965 & -1.0489 & H & 0.4063 & 0.9675 & 0.2962 & 0 & -1.9285 & 6.5184 & -4.9190 \\
\hline $\mathrm{H}$ & -4.6357 & 0.4897 & -3.6563 & $\mathrm{H}$ & -5.6618 & 3.7512 & 0.1157 & $\mathrm{~N}$ & -2.9049 & 4.8773 & -3.6469 \\
\hline $\mathrm{H}$ & -6.9367 & 0.0962 & -0.0589 & H & -4.8954 & 4.4994 & -1.3421 & C & -3.4135 & 4.0911 & -4.7563 \\
\hline $\mathrm{H}$ & -4.8126 & -0.9459 & -0.4617 & H & -5.8206 & 2.9911 & -1.4638 & C & -2.7565 & 2.7089 & -4.8566 \\
\hline C & -0.3653 & -7.1105 & -4.4288 & H & -2.8770 & 0.1684 & 1.1333 & C & -1.2502 & 2.7026 & -5.1618 \\
\hline C & -1.5057 & -7.7034 & -3.5856 & $\mathrm{H}$ & -1.7808 & -2.0003 & -0.3811 & C & -0.7045 & 1.2747 & -5.0487 \\
\hline C & -2.3610 & -6.6390 & -2.9386 & $\mathrm{H}$ & -2.1593 & -2.6157 & -1.9892 & c & -0.9320 & 3.3263 & -6.5255 \\
\hline C & -2.0313 & -6.1170 & -1.6834 & $\mathrm{H}$ & -2.5834 & -0.9656 & -1.5767 & C & 3.6359 & 1.3566 & -2.1464 \\
\hline C & -3.4719 & -6.1001 & -3.5989 & $\mathrm{H}$ & 0.6962 & -3.9032 & -3.5743 & C & 4.3873 & 2.3830 & -1.5166 \\
\hline C & -2.7689 & -5.0892 & -1.0971 & $\mathrm{H}$ & -0.2096 & -2.9789 & -4.7785 & C & 5.7178 & 2.6221 & -1.8401 \\
\hline C & -4.2240 & -5.0770 & -3.0313 & C & 1.7396 & -2.0575 & 7.6539 & C & 6.3631 & 1.8576 & -2.8177 \\
\hline C & -3.8750 & -4.5645 & -1.7767 & C & 0.9156 & -0.8052 & 7.3423 & C & 5.6418 & 0.8547 & -3.4633 \\
\hline 0 & -4.6391 & -3.5553 & -1.2828 & C & 1.7016 & 0.1334 & 6.4268 & C & 4.3084 & 0.6071 & -3.1404 \\
\hline $\mathrm{H}$ & 0.3040 & -6.5086 & -3.8052 & 0 & 1.9757 & -0.3400 & 5.2471 & C & 2.2681 & 1.1404 & -1.6704 \\
\hline $\mathrm{H}$ & -2.1312 & -8.3417 & -4.2218 & 0 & 2.0515 & 1.2512 & 6.8300 & 0 & 1.7785 & 2.1654 & -0.8758 \\
\hline $\mathrm{H}$ & -1.0804 & -8.3547 & -2.8126 & C & -8.4263 & 3.0953 & 3.7889 & $\mathrm{H}$ & 2.2028 & 7.6779 & -3.2330 \\
\hline $\mathrm{H}$ & -1.1770 & -6.5203 & -1.1528 & C & -7.5182 & 1.9071 & 3.4719 & $\mathrm{H}$ & 2.7842 & 6.0056 & -3.0040 \\
\hline $\mathrm{H}$ & -3.7490 & -6.4843 & -4.5779 & C & -6.6108 & 2.1611 & 2.2648 & $\mathrm{H}$ & -1.7019 & 5.9175 & -1.6944 \\
\hline $\mathrm{H}$ & -2.4796 & -4.6768 & -0.1336 & 0 & -6.6468 & 3.3013 & 1.7420 & $\mathrm{H}$ & -2.2300 & 7.4959 & -2.2894 \\
\hline $\mathrm{H}$ & -5.0785 & -4.6504 & -3.5464 & 0 & -5.8686 & 1.1912 & 1.8890 & $\mathrm{H}$ & -2.9330 & 2.1739 & -3.9161 \\
\hline $\mathrm{H}$ & -4.2526 & -3.1205 & -0.4899 & H & -9.0340 & 2.8965 & 4.6797 & $\mathrm{H}$ & -3.2849 & 2.1404 & -5.6365 \\
\hline C & 4.8550 & -4.7453 & -5.0871 & $\mathrm{H}$ & -7.8386 & 3.9965 & 3.9660 & $\mathrm{H}$ & -0.7438 & 3.3102 & -4.4077 \\
\hline C & 5.1231 & -5.3155 & -3.7104 & H & -8.0997 & 0.9974 & 3.2758 & $\mathrm{H}$ & 0.1396 & 3.2511 & -6.7393 \\
\hline 0 & 6.2591 & -5.6235 & -3.3490 & $\mathrm{H}$ & -6.8670 & 1.6624 & 4.3205 & $\mathrm{H}$ & -1.4694 & 2.8122 & -7.3336 \\
\hline C & 4.8746 & -3.2090 & -5.1409 & C & -0.9280 & 2.4917 & 5.2639 & $\mathrm{H}$ & -1.1982 & 4.3869 & -6.5506 \\
\hline $\mathrm{H}$ & 5.6338 & -5.1468 & -5.7414 & $\mathrm{C}$ & -0.7496 & 1.7696 & 3.9182 & $\mathrm{H}$ & -1.1667 & 0.6129 & -5.7928 \\
\hline $\mathrm{H}$ & 5.8037 & -2.8161 & -4.7232 & C & 0.5089 & 2.1217 & 3.1877 & $\mathrm{H}$ & -0.9021 & 0.8546 & -4.0580 \\
\hline $\mathrm{H}$ & 4.0562 & -2.7807 & -4.5579 & C & 0.7107 & 2.7541 & 1.9800 & $\mathrm{H}$ & 0.3781 & 1.2480 & -5.2047 \\
\hline $\mathrm{H}$ & 4.7768 & -2.8713 & -6.1774 & $\mathrm{~N}$ & 1.7733 & 1.7900 & 3.6319 & $\mathrm{H}$ & -3.2551 & 4.6772 & -5.6630 \\
\hline $\mathrm{N}$ & 4.0193 & -5.4959 & -2.9256 & C & 2.6653 & 2.1887 & 2.6949 & $\mathrm{H}$ & 3.9165 & 3.0057 & -0.7680 \\
\hline C & 4.2088 & -5.7214 & -1.5039 & $\mathrm{~N}$ & 2.0588 & 2.7759 & 1.6667 & $\mathrm{H}$ & 6.2534 & 3.4121 & -1.3224 \\
\hline C & 2.9977 & -6.3082 & -0.7793 & $\mathrm{H}$ & -0.0952 & 2.2773 & 5.9397 & $\mathrm{H}$ & 7.3989 & 2.0505 & -3.0773 \\
\hline C & 1.7158 & -5.4461 & -0.7324 & $\mathrm{H}$ & -0.7825 & 0.6858 & 4.0772 & $\mathrm{H}$ & 6.1168 & 0.2421 & -4.2241 \\
\hline C & 0.8023 & -5.9406 & 0.3962 & $\mathrm{H}$ & -1.5922 & 1.9979 & 3.2569 & $\mathrm{H}$ & 3.8096 & -0.1815 & -3.6835 \\
\hline C & 2.0092 & -3.9597 & -0.5337 & $\mathrm{H}$ & -0.0286 & 3.1756 & 1.3147 & C & -5.1080 & -2.8591 & 3.8230 \\
\hline $\mathrm{H}$ & 3.1946 & -4.9764 & -3.1947 & $\mathrm{H}$ & 3.7324 & 2.0614 & 2.7995 & C & -3.6730 & -2.2834 & 3.8552 \\
\hline $\mathrm{H}$ & 4.5192 & -4.7870 & -1.0241 & $\mathrm{H}$ & 2.1475 & 2.3077 & 0.0472 & 0 & -3.4928 & -1.0796 & 4.0268 \\
\hline $\mathrm{H}$ & 3.3234 & -6.4815 & 0.2549 & C & 5.3282 & 7.3922 & 2.2830 & C & -6.0066 & -2.0305 & 2.8861 \\
\hline $\mathrm{H}$ & 2.7549 & -7.2929 & -1.1988 & C & 5.2212 & 6.2009 & 3.2395 & C & -5.6751 & -2.2980 & 1.4369 \\
\hline $\mathrm{H}$ & 1.1787 & -5.5636 & -1.6862 & C & 5.9093 & 4.9138 & 2.7489 & $\mathrm{~N}$ & -6.6334 & -2.8262 & 0.6642 \\
\hline $\mathrm{H}$ & 1.3100 & -5.8225 & 1.3606 & C & 5.8562 & 3.8395 & 3.8446 & 0 & -4.5376 & -2.0267 & 0.9775 \\
\hline $\mathrm{H}$ & -0.1174 & -5.3508 & 0.4397 & C & 5.2993 & 4.3912 & 1.4406 & $\mathrm{~N}$ & -2.6276 & -3.1562 & 3.7791 \\
\hline $\mathrm{H}$ & 0.5398 & -6.9993 & 0.2833 & H & 4.8134 & 7.1979 & 1.3368 & C & -1.2625 & -2.7128 & 4.0612 \\
\hline $\mathrm{H}$ & 2.6184 & -3.5505 & -1.3413 & H & 4.1623 & 5.9806 & 3.4350 & C & -0.5041 & -2.2143 & 2.8184 \\
\hline $\mathrm{H}$ & 2.5440 & -3.8053 & 0.4100 & $\mathrm{H}$ & 5.6576 & 6.4805 & 4.2078 & 0 & -0.8464 & -2.5228 & 1.6719 \\
\hline $\mathrm{H}$ & 1.0812 & -3.3839 & -0.4875 & $\mathrm{H}$ & 6.9643 & 5.1498 & 2.5542 & C & -0.5837 & -4.0088 & 4.5544 \\
\hline C & 9.1431 & -5.4126 & -0.2780 & $\mathrm{H}$ & 4.8203 & 3.6146 & 4.1229 & C & -1.2437 & -5.0750 & 3.6675 \\
\hline C & 7.8963 & -4.5660 & -0.5857 & $\mathrm{H}$ & 6.3221 & 2.9029 & 3.5202 & C & -2.7046 & -4.6064 & 3.5552 \\
\hline C & 8.2476 & -3.2045 & -1.1396 & $\mathrm{H}$ & 6.3712 & 4.1722 & 4.7533 & $\mathrm{~N}$ & 0.6152 & -1.5168 & 3.1163 \\
\hline C & 8.1399 & -2.9444 & -2.5126 & $\mathrm{H}$ & 4.2176 & 4.2463 & 1.5377 & C & 1.6334 & -1.2252 & 2.1309 \\
\hline C & 8.6971 & -2.1788 & -0.2990 & $\mathrm{H}$ & 5.7454 & 3.4314 & 1.1642 & C & 3.0035 & -1.7479 & 2.5815 \\
\hline C & 8.4610 & -1.6865 & -3.0225 & $\mathrm{H}$ & 5.4711 & 5.0836 & 0.6097 & 0 & 3.1784 & -2.9166 & 2.9138 \\
\hline C & 9.0160 & -0.9161 & -0.8037 & $\mathrm{H}$ & -1.0300 & -3.6329 & -3.3510 & $\mathrm{~N}$ & 3.9973 & -0.8170 & 2.5343 \\
\hline C & 8.8963 & -0.6667 & -2.1716 & $\mathrm{H}$ & -9.0999 & 3.2937 & 2.9554 & C & 5.3884 & -1.0832 & 2.8405 \\
\hline $\mathrm{H}$ & 9.7916 & -4.9151 & 0.4510 & $\mathrm{H}$ & 11.9892 & 4.4157 & -1.0995 & C & 5.8837 & -0.3262 & 4.0885 \\
\hline $\mathrm{H}$ & 7.3104 & -4.4447 & 0.3349 & $\mathrm{H}$ & 11.5482 & 5.9359 & -1.9057 & 0 & 7.0862 & -0.0772 & 4.2203 \\
\hline $\mathrm{H}$ & 7.2665 & -5.0916 & -1.3084 & $\mathrm{H}$ & -10.3819 & 2.2808 & -5.0842 & C & 6.3352 & -0.7607 & 1.6872 \\
\hline $\mathrm{H}$ & 7.7674 & -3.7281 & -3.1651 & $\mathrm{H}$ & -8.8907 & 1.3689 & -5.3822 & 0 & 6.1316 & 0.6049 & 1.2788 \\
\hline
\end{tabular}




\begin{tabular}{|c|c|c|c|}
\hline $\mathrm{N}$ & 4.9511 & 0.0165 & 4.9919 \\
\hline C & 5.3695 & 0.6527 & 6.2248 \\
\hline $\mathrm{H}$ & -7.0597 & -2.2558 & 0768 \\
\hline $\mathrm{H}$ & -5.8295 & -0.9626 & 3.0552 \\
\hline $\mathrm{H}$ & -5.1351 & -3.9163 & 3.5507 \\
\hline $\mathrm{H}$ & -0.8257 & -4.1683 & 5.6106 \\
\hline $\mathrm{H}$ & 0.5025 & -3.9702 & 4.4469 \\
\hline$T$ & -1.1660 & -6.0860 & 4.0748 \\
\hline $\mathrm{H}$ & -0.7769 & -5.0655 & 2.6808 \\
\hline $\mathrm{H}$ & -3.3306 & -5.0833 & 4.3202 \\
\hline $\mathrm{H}$ & -3.1327 & -4.8307 & 2.5728 \\
\hline$\Pi$ & -1.2854 & -1.9231 & 4.8158 \\
\hline $\mathrm{H}$ & 1.6788 & -0.1547 & 1.9198 \\
\hline H & 1.3651 & -1.7435 & 1.2081 \\
\hline $\mathrm{H}$ & 7.3541 & -0.8814 & 2.0580 \\
\hline $\mathrm{H}$ & 6.1859 & -1.4435 & 0.8548 \\
\hline $\mathrm{H}$ & 6.1434 & 0.6639 & 0.3088 \\
\hline $\mathrm{H}$ & 5.4532 & -2.1551 & 3.0599 \\
\hline H & 6.0861 & 1.4470 & 6.0030 \\
\hline $\mathrm{H}$ & 2.1518 & 6.5060 & -4.5730 \\
\hline $\mathrm{H}$ & -4.4965 & 3.9608 & -4.6303 \\
\hline $\mathrm{H}$ & -2.9025 & 4.4165 & -2.7334 \\
\hline $\mathrm{H}$ & -0.0788 & 8.0122 & -2.9213 \\
\hline H & 5.8594 & -0.0618 & 6.8978 \\
\hline $\mathrm{H}$ & 4.4846 & 1.0707 & 6.7079 \\
\hline $\mathrm{H}$ & 3.9675 & -0.2382 & 4.8900 \\
\hline $\mathrm{H}$ & 0.8686 & -1.2590 & 4.0799 \\
\hline H & -7.4908 & -3.2188 & 1.0517 \\
\hline $\mathrm{H}$ & -6.3615 & -3.1129 & -0.2711 \\
\hline H & 3.7690 & 0.1123 & 2.2139 \\
\hline $\mathrm{H}$ & -5.4890 & -2.7722 & 4.8462 \\
\hline $\mathrm{C}$ & -11.0909 & -4.9554 & 2.2288 \\
\hline C & -10.0870 & -4.4072 & 1.2388 \\
\hline 0 & -8.9107 & -4.2302 & 1.5682 \\
\hline C & -11.2732 & -3.9152 & 3.3376 \\
\hline $\mathrm{N}$ & -10.5648 & -4.0533 & 0.0257 \\
\hline $\mathrm{C}$ & -9.7259 & -3.4001 & -0.9616 \\
\hline C & -9.0166 & 7.2363 & 2.5275 \\
\hline C & -8.8783 & 5.8480 & 1.9209 \\
\hline C & -8.8964 & 5.8174 & 0.3950 \\
\hline $\mathrm{N}$ & -8.4181 & 4.6480 & -0.1244 \\
\hline 0 & -9.3537 & 6.7253 & -0.2931 \\
\hline $\mathrm{H}$ & -10.3078 & -3.6884 & 3.7951 \\
\hline $\mathrm{H}$ & -11.9506 & -4.2804 & 4.1145 \\
\hline $\mathrm{H}$ & -11.6867 & -2.9842 & 2.9372 \\
\hline $\mathrm{H}$ & -12.0471 & -5.1932 & 1.7493 \\
\hline $\mathrm{H}$ & -9.3070 & -2.4680 & -0.5711 \\
\hline $\mathrm{H}$ & -7.9715 & 5.3456 & 2.2647 \\
\hline $\mathrm{H}$ & -9.7125 & 5.2187 & 2.2544 \\
\hline $\mathrm{H}$ & -7.8497 & 4.0295 & 0.4543 \\
\hline $\mathrm{H}$ & -8.3200 & 4.5981 & -1.1275 \\
\hline $\mathrm{H}$ & -9.9016 & 7.7433 & 2.1341 \\
\hline $\mathrm{H}$ & -9.0979 & 7.1750 & 3.6171 \\
\hline $\mathrm{H}$ & -8.1513 & 7.8619 & 2.2861 \\
\hline $\mathrm{H}$ & -10.6833 & -5.8826 & 2.6422 \\
\hline $\mathrm{H}$ & -10.3250 & -3.1737 & -1.8442 \\
\hline $\mathrm{H}$ & -8.8949 & -4.0480 & -1.2574 \\
\hline $\mathrm{H}$ & -11.5371 & -4.2151 & -0.1817 \\
\hline C & 5.1508 & -1.8229 & -1.5507 \\
\hline $\mathrm{H}$ & 6.0984 & -1.2531 & -1.5788 \\
\hline$v$ & 4.9985 & -2.7855 & -2.2780 \\
\hline C & 4.1139 & -1.4010 & -0.5541 \\
\hline 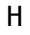 & 4.1207 & -2.1250 & 0.2628 \\
\hline $\mathrm{H}$ & 4.3101 & -0.4086 & -0.1566 \\
\hline $\mathrm{H}$ & 3.1264 & -1.4336 & -1.0071 \\
\hline
\end{tabular}

En(AA)-R1

$\begin{array}{rrrr}\text { C } & 11.4734 & 5.0513 & -0.4710 \\ \mathrm{C} & 9.9607 & 5.0564 & -0.1962 \\ \mathrm{C} & 9.4582 & 3.8201 & 0.4925 \\ \mathrm{C} & 8.4413 & 2.9738 & 0.1084 \\ \mathrm{~N} & 9.9769 & 3.4052 & 1.7069 \\ \mathrm{C} & 9.2769 & 2.3353 & 2.0412 \\ \mathrm{~N} & 8.3378 & 2.0228 & 1.1050 \\ \mathrm{H} & 12.0207 & 4.8935 & 0.4622 \\ \mathrm{H} & 9.4084 & 5.1892 & -1.1343 \\ \mathrm{H} & 9.7217 & 5.9285 & 0.4277 \\ \mathrm{H} & 7.7824 & 2.9798 & -0.7450 \\ \mathrm{H} & 9.3977 & 1.7526 & 2.9437\end{array}$

\begin{tabular}{|c|c|c|c|c|c|c|c|}
\hline H & 7.6109 & 1.3065 & 1.1956 & C & -3.5101 & 2.8541 & -0.080 \\
\hline C & -9.4903 & 2.4062 & -4.2323 & $\mathrm{~N}$ & -3.4921 & 1.7600 & $0.71 \varepsilon$ \\
\hline C & -8.7030 & 3.1278 & -3.1244 & $\mathrm{~S}$ & 1.4615 & -0.9948 & -3.376 \\
\hline C & -7.6032 & 2.2584 & -2.5603 & C & 1.4352 & 0.0032 & -1.977 \\
\hline C & -6.4518 & 1.9976 & -3.3185 & C & -4.7558 & 3.6644 & -0.185 \\
\hline C & -7.7191 & 1.6356 & -1.3122 & $\mathrm{~N}$ & 0.2968 & -0.2472 & -1.316 \\
\hline C & -5.4674 & 1.1311 & -2.8622 & C & -0.5554 & -1.2403 & -1.84 \\
\hline C & -6.7363 & 0.7675 & -0.8311 & C & -1.8235 & -1.6288 & -1.181 \\
\hline C & -5.6094 & 0.5071 & -1.6164 & C & -0.0559 & -1.7639 & -2.996 \\
\hline 0 & -4.5977 & -0.3067 & -1.2303 & C & -0.6210 & -2.8589 & -3.84 \\
\hline $\mathrm{H}$ & -9.9513 & 1.4922 & -3.8437 & C & -0.0307 & 0.3940 & 0.00 \\
\hline$\pi$ & -9.3858 & 3.4289 & -2.3218 & $\mathrm{H}$ & 0.7237 & 2.6513 & -1.26 \\
\hline $\mathrm{H}$ & -8.2767 & 4.0524 & -3.5338 & $\mathrm{H}$ & -0.3478 & 3.8802 & -1.83 \\
\hline $\mathrm{H}$ & -6.3277 & 2.4783 & -4.2860 & $\mathrm{H}$ & -0.1651 & -0.4120 & 0.71 \\
\hline $\mathrm{H}$ & -8.5825 & 1.8525 & -0.6886 & $\mathrm{H}$ & 0.8493 & 0.9695 & 0 \\
\hline $\mathrm{H}$ & -4.5792 & 0.9256 & -3.4487 & $\mathrm{H}$ & -5.2646 & 3.7115 & 0.781 \\
\hline $\mathrm{H}$ & -6.8023 & 0.3504 & 0.1682 & $\mathrm{H}$ & -4.5088 & 4.6581 & -0.55 \\
\hline $\mathrm{H}$ & -4.7675 & -0.7794 & -0.3932 & $\mathrm{H}$ & -5.4369 & 3.1861 & -0.89 \\
\hline C & -0.4512 & -6.5236 & -5.3985 & $\mathrm{H}$ & -2.4673 & 0.0695 & \\
\hline C & -1.2728 & -7.1853 & -4.2794 & $\mathrm{H}$ & -1.6717 & -1.9064 & -0.13 \\
\hline C & -2.2035 & -6.2191 & -3.5801 & $\mathrm{H}$ & -2.2220 & -2.4985 & -1.69 \\
\hline C & -2.0214 & -5.8517 & -2.2431 & $\mathrm{H}$ & -2.5676 & -0.8379 & -1.24 \\
\hline C & -3.2698 & -5.6318 & -4.2763 & $\mathrm{H}$ & -0.2938 & -3.8421 & -3.492 \\
\hline C & -2.8492 & -4.9181 & -1.6157 & $\mathrm{H}$ & -0.3086 & -2.7498 & -4.88 \\
\hline C & -4.1025 & -4.6975 & -3.6734 & C & 1.8486 & -3.2545 & 7.25 \\
\hline C & -3.8904 & -4.3234 & -2.3397 & C & 1.1019 & -1.9235 & 7.34 \\
\hline 0 & -4.7095 & -3.3707 & -1.8251 & C & 1.8408 & -0.8370 & 6.557 \\
\hline $\mathrm{H}$ & 0.1830 & -5.7282 & -4.9939 & 0 & 2.0200 & -1.0999 & 5.29 \\
\hline $\mathrm{H}$ & -1.8515 & -8.0142 & -4.7080 & 0 & 2.2247 & 0.1933 & 7.12 \\
\hline $\mathrm{H}$ & -0.5935 & -7.6329 & -3.5450 & C & -8.2494 & 2.5689 & 4.2 \\
\hline $\mathrm{H}$ & -1.2096 & -6.2974 & -1.6746 & C & -7.2874 & 1.4814 & 3.761 \\
\hline $\mathrm{H}$ & -3.4443 & -5.9035 & -5.3146 & C & -6.3131 & 1.9506 & 2.66 \\
\hline $\mathrm{H}$ & -2.6679 & -4.6257 & -0.5850 & 0 & -6.3046 & 3.1698 & 2.37 \\
\hline $\mathrm{H}$ & -4.9148 & -4.2287 & -4.2191 & 0 & -5.5633 & 1.0523 & ? \\
\hline $\mathrm{H}$ & -4.3899 & -3.0103 & -0.9582 & $\mathrm{H}$ & -8.8797 & 2.1867 & 5.06 \\
\hline C & 4.8075 & -4.1819 & -5.7748 & $\mathrm{H}$ & -7.6991 & 3.4355 & 4.62 \\
\hline C & 5.0919 & -4.9300 & -4.4937 & $\mathrm{H}$ & -7.8352 & 0.6138 & 3.37 \\
\hline 0 & 6.2153 & -5.3306 & -4.1828 & $\mathrm{H}$ & -6.6750 & 1.0925 & \\
\hline C & 4.5535 & -2.6847 & -5.5201 & C & -0.7516 & 1.6316 & 5.55 \\
\hline $\mathrm{H}$ & 5.6749 & -4.3155 & -6.4246 & C & -0.6286 & 1.3010 & 4.05 \\
\hline $\mathrm{H}$ & 5.4008 & -2.2336 & -4.9970 & C & 0.6912 & 1.6769 & 3.45 \\
\hline $\mathrm{H}$ & 3.6612 & -2.5191 & -4.9091 & C & 1.0396 & 610 & \\
\hline $\mathrm{H}$ & 4.4126 & -2.1481 & -6.4628 & $\mathrm{~N}$ & 1.8895 & 1.2009 & 3.94 \\
\hline N & 4.0151 & -5.0883 & -3.6859 & C & 2.8812 & 1.6804 & 3.15 \\
\hline C & 4.1723 & -5.6404 & -2.3576 & $\mathrm{~N}$ & 2.4091 & 2.4487 & 2 \\
\hline C & 2.8844 & -5.5559 & -1.5456 & $\mathrm{H}$ & 0.0549 & 1.1775 & $6.13]$ \\
\hline C & 2.3162 & -4.1396 & -1.3397 & $\mathrm{H}$ & -0.8041 & 0.2277 & 3.90 \\
\hline C & 1.1021 & -4.2113 & -0.4097 & $\mathrm{H}$ & -1.4173 & 1.8145 & 3.49 \\
\hline C & 3.3672 & -3.1615 & -0.7982 & $\mathrm{H}$ & 0.3907 & 3.0334 & \\
\hline $\mathrm{H}$ & 3.1296 & -4.6816 & -3.9447 & $\mathrm{H}$ & 3.9220 & 1.4447 & 3.32 \\
\hline $\mathrm{H}$ & 4.9761 & -5.1071 & -1.8412 & $\mathrm{H}$ & 3.2226 & 1.5665 & 0.59 \\
\hline $\mathrm{H}$ & 3.0870 & -5.9962 & -0.5616 & C & 5.5720 & 6.7906 & 3.218 \\
\hline $\mathrm{H}$ & 2.1130 & -6.1852 & -2.0123 & C & 5.6502 & 5.4656 & \\
\hline $\mathrm{H}$ & 1.9694 & -3.7575 & -2.3118 & C & 6.2358 & 4.2821 & 3.1 \\
\hline $\mathrm{H}$ & 1.4136 & -4.4508 & 0.6127 & C & 6.2696 & 3.0311 & 4.07 \\
\hline $\mathrm{H}$ & 0.5605 & -3.2665 & -0.3716 & C & 5.4554 & 4.0179 & 1.89 \\
\hline $\mathrm{H}$ & 0.3900 & -4.9742 & -0.7413 & $\mathrm{H}$ & 4.8854 & 6.7237 & \\
\hline $\mathrm{H}$ & 4.1908 & -3.0050 & -1.5023 & $\mathrm{H}$ & 4.6427 & 5.1873 & 4.32 \\
\hline $\mathrm{H}$ & 3.7858 & -3.5348 & 0.1418 & $\mathrm{H}$ & 6.2496 & 5.6090 & 4.89 \\
\hline $\mathrm{H}$ & 2.9238 & -2.1829 & -0.5923 & $\mathrm{H}$ & 7.2723 & 4.5274 & 2.91 \\
\hline C & 9.1218 & -5.5933 & -1.1534 & $\mathrm{H}$ & 5.2702 & 2.8030 & 4.46 \\
\hline C & 7.7971 & -4.8171 & -1.1631 & $\mathrm{H}$ & 6.6220 & 2.1528 & 3.53 \\
\hline C & 7.9505 & -3.3153 & -1.3038 & $\mathrm{H}$ & 6.9276 & 3.1783 & 4.94 \\
\hline C & 7.2711 & -2.6307 & -2.3200 & $\mathrm{H}$ & 4.3853 & 3.8853 & 2.08 \\
\hline C & 8.7492 & -2.5724 & -0.4238 & $\mathrm{H}$ & 5.8215 & 3.1085 & 1.41 \\
\hline C & 7.3768 & -1.2438 & -2.4478 & $\mathrm{H}$ & 5.5785 & 4.8345 & 1.17 \\
\hline C & 8.8687 & -1.1887 & -0.5547 & $\mathrm{H}$ & -1.7124 & -2.8591 & -3.82 \\
\hline C & 8.1792 & -0.5173 & -1.5666 & $\mathrm{H}$ & -8.9006 & 2.9018 & 3.44 \\
\hline $\mathrm{H}$ & 9.7699 & -5.2894 & -0.3261 & $\mathrm{H}$ & 11.7432 & 4.2410 & -1.15 \\
\hline $\mathrm{H}$ & 7.2530 & -5.0338 & -0.2327 & $\mathrm{H}$ & 11.7982 & 5.9993 & -0.91 \\
\hline $\mathrm{H}$ & 7.1801 & -5.1903 & -1.9865 & $\mathrm{H}$ & -10.2819 & 3.0440 & -4.64 \\
\hline $\mathrm{H}$ & 6.6848 & 2122 & -3.0236 & $\mathrm{H}$ & -8.8277 & 2.1175 & -5.05 \\
\hline $\mathrm{H}$ & 9.2709 & -3.0791 & 0.3840 & $\mathrm{H}$ & -0.7003 & 2.7132 & 5.71 \\
\hline $\mathrm{H}$ & 6.8503 & -0.7334 & -3.2503 & $\mathrm{H}$ & -1.7088 & 1.2709 & 5.93 \\
\hline $\mathrm{H}$ & 9.4795 & -0.6253 & 0.1442 & $\mathrm{H}$ & 6.5552 & 7.0796 & 2.82 \\
\hline $\mathrm{H}$ & 2758 & 589 & -1.6621 & $\mathrm{H}$ & 5.2192 & 7.6004 & 3.86 \\
\hline C & -2.3905 & 0.9566 & 0.7317 & $\mathrm{H}$ & 0.0960 & -2.0326 & 6.91 \\
\hline C & -1.2706 & 1.2564 & -0.0041 & $\mathrm{H}$ & 0.9899 & -1.5953 & 8.37 \\
\hline C & -1.3079 & 2.4950 & -0.7299 & $\mathrm{H}$ & 2.0022 & -3.5297 & 6.20 \\
\hline $\mathrm{N}$ & 2671 & 2.9765 & -1.3763 & $\mathrm{H}$ & 1.3045 & -4.0632 & 7.7 \\
\hline r & -2.4572 & 3.2287 & -0.7779 & $\mathrm{H}$ & 2.8361 & -3.1763 & 7.71 \\
\hline
\end{tabular}




\begin{tabular}{|c|c|c|c|c|c|c|c|c|c|c|c|}
\hline $\mathrm{H}$ & 8.9353 & -6.6675 & -1.0556 & $\mathrm{H}$ & -3.2757 & -5.7317 & 4.0508 & 0 & -4.6847 & -0.6041 & -1.3692 \\
\hline $\mathrm{H}$ & 9.6699 & -5.4277 & -2.0864 & $\mathrm{H}$ & -3.1036 & -5.3063 & 2.3342 & $\mathrm{H}$ & -10.0061 & 0.8382 & -4.1206 \\
\hline $\mathrm{H}$ & 4.5003 & -6.6840 & -2.4374 & $\mathrm{H}$ & -1.2862 & -2.6064 & 4.8546 & $\mathrm{H}$ & -9.5234 & 2.9420 & -2.8095 \\
\hline $\mathrm{H}$ & 0.1942 & -7.2519 & -5.9003 & $\mathrm{H}$ & 1.6783 & -0.5448 & 2.1081 & $\mathrm{H}$ & -8.4189 & 3.4709 & -4.0725 \\
\hline $\mathrm{H}$ & -1.1030 & -6.0719 & -6.1525 & $\mathrm{H}$ & 1.2174 & -1.9953 & 1.1996 & $\mathrm{H}$ & -6.4083 & 1.9281 & -4.6371 \\
\hline $\mathrm{H}$ & 3.9389 & -4.6232 & -6.2781 & $\mathrm{H}$ & 7.2129 & -1.4831 & 1.6917 & $\mathrm{H}$ & -8.7229 & 1.4965 & -1.0489 \\
\hline $\mathrm{H}$ & 2.0085 & 0.4289 & 4.6380 & $\mathrm{H}$ & 5.8784 & -1.6098 & 0.5239 & $\mathrm{H}$ & -4.6357 & 0.4897 & -3.6563 \\
\hline $\mathrm{H}$ & -4.3799 & 1.5014 & 1.3207 & $\mathrm{H}$ & 5.2577 & 0.4919 & 1.1207 & $\mathrm{H}$ & -6.9367 & 0.0962 & -0.0589 \\
\hline C & 2.2155 & 6.9049 & -2.5710 & $\mathrm{H}$ & 5.3784 & -2.9076 & 2.5812 & $\mathrm{H}$ & -4.8126 & -0.9459 & -0.4617 \\
\hline C & 0.8235 & 6.3883 & -2.3353 & $\mathrm{H}$ & 6.3019 & 0.3115 & 6.0267 & C & -0.3653 & -7.1105 & -4.4288 \\
\hline 0 & 0.5002 & 5.2552 & -2.7048 & $\mathrm{H}$ & 2.3803 & 6.9846 & -3.6492 & C & -1.5057 & -7.7034 & -3.5856 \\
\hline $\mathrm{N}$ & -0.0524 & 7.1984 & -1.7008 & $\mathrm{H}$ & -4.3836 & 4.6389 & -4.0317 & C & -2.3610 & -6.6390 & -2.9386 \\
\hline C & -1.4404 & 6.7902 & -1.5655 & $\mathrm{H}$ & -2.6625 & 4.6438 & -2.0981 & C & -2.0313 & -6.1170 & -1.6834 \\
\hline C & -2.0300 & 6.4065 & -2.9363 & $\mathrm{H}$ & 0.1936 & 8.1637 & -1.5528 & C & -3.4719 & -6.1001 & -3.5989 \\
\hline 0 & -1.8709 & 7.1321 & -3.9135 & $\mathrm{H}$ & 5.9472 & -1.2594 & 6.7581 & C & -2.7689 & -5.0892 & -1.0971 \\
\hline $\mathrm{N}$ & -2.7278 & 5.2512 & -2.9179 & $\mathrm{H}$ & 4.6801 & 0.0037 & 6.7207 & C & -4.2240 & -5.0770 & -3.0313 \\
\hline C & -3.2885 & 4.6652 & -4.1194 & $\mathrm{H}$ & 4.0174 & -1.0137 & 4.7838 & C & -3.8750 & -4.5645 & -1.7767 \\
\hline C & -2.7844 & 3.2379 & -4.3635 & $\mathrm{H}$ & 0.8520 & -1.8375 & 4.1240 & 0 & -4.6391 & -3.5553 & -1.2828 \\
\hline C & -1.2989 & 3.1037 & -4.7306 & $\mathrm{H}$ & -7.4624 & -3.5770 & 0.7129 & $\mathrm{H}$ & 0.3040 & -6.5086 & -3.8052 \\
\hline C & -0.8893 & 1.6266 & -4.7272 & $\mathrm{H}$ & -6.3673 & -3.3762 & -0.6247 & $\mathrm{H}$ & -2.1312 & -8.3417 & -4.2218 \\
\hline C & -0.9817 & 3.7687 & -6.0749 & $\mathrm{H}$ & 3.7272 & -0.5161 & 2.1134 & $\mathrm{H}$ & -1.0804 & -8.3547 & -2.8126 \\
\hline C & 3.0115 & 1.7657 & -3.2704 & $\mathrm{H}$ & -5.4628 & -3.4516 & 4.4303 & $\mathrm{H}$ & -1.1770 & -6.5203 & -1.1528 \\
\hline C & 2.4757 & 2.9258 & -3.8345 & C & -11.2331 & -5.0853 & 1.5683 & $\mathrm{H}$ & -3.7490 & -6.4843 & -4.5779 \\
\hline C & 2.6710 & 3.2059 & -5.1870 & C & -10.1762 & -4.5113 & 0.6504 & $\mathrm{H}$ & -2.4796 & -4.6768 & -0.1336 \\
\hline C & 3.4029 & 2.3319 & -5.9915 & 0 & -8.9813 & -4.5670 & 0.9549 & $\mathrm{H}$ & -5.0785 & -4.6504 & -3.5464 \\
\hline C & 3.9572 & 1.1794 & -5.4299 & C & -11.2322 & -4.2576 & 2.8568 & $\mathrm{H}$ & -4.2526 & -3.1205 & -0.4899 \\
\hline C & 3.7690 & 0.9029 & -4.0774 & $\mathrm{~N}$ & -10.6177 & -3.8775 & -0.4579 & C & 4.8550 & -4.7453 & -5.0871 \\
\hline C & 2.8021 & 1.5172 & -1.7852 & C & -9.7108 & -3.1717 & -1.3443 & C & 5.1231 & -5.3155 & -3.7104 \\
\hline 0 & 2.2605 & 2.4293 & -1.0851 & C & -8.7707 & 6.8555 & 3.5936 & 0 & 6.2591 & -5.6235 & -3.3490 \\
\hline $\mathrm{H}$ & 2.4076 & 7.8756 & -2.1071 & C & -8.6345 & 5.5607 & 2.8076 & C & 4.8746 & -3.2090 & -5.1409 \\
\hline $\mathrm{H}$ & 2.9248 & 6.1712 & -2.1804 & C & -8.6290 & 5.7362 & 1.2918 & $\mathrm{H}$ & 5.6338 & -5.1468 & -5.7414 \\
\hline $\mathrm{H}$ & -1.5221 & 5.9634 & -0.8528 & $\mathrm{~N}$ & -8.0955 & 4.6656 & 0.6311 & $\mathrm{H}$ & 5.8037 & -2.8161 & -4.7232 \\
\hline $\mathrm{H}$ & -2.0048 & 7.6369 & -1.1682 & 0 & -9.1107 & 6.7107 & 0.7219 & $\mathrm{H}$ & 4.0562 & -2.7807 & -4.5579 \\
\hline $\mathrm{H}$ & -2.9907 & 2.6443 & -3.4652 & $\mathrm{H}$ & -10.2346 & -4.2659 & 3.3010 & $\mathrm{H}$ & 4.7768 & -2.8713 & -6.1774 \\
\hline $\mathrm{H}$ & -3.3934 & 2.7969 & -5.1663 & $\mathrm{H}$ & -11.9421 & -4.6591 & 3.5853 & $\mathrm{~N}$ & 4.0193 & -5.4959 & -2.9256 \\
\hline $\mathrm{H}$ & -0.7088 & 3.6179 & -3.9679 & $\mathrm{H}$ & -11.5061 & -3.2174 & 2.6546 & C & 4.2088 & -5.7214 & -1.5039 \\
\hline $\mathrm{H}$ & 0.0604 & 3.5894 & -6.3553 & $\mathrm{H}$ & -12.2234 & -5.0859 & 1.0994 & C & 2.9977 & -6.3082 & -0.7793 \\
\hline $\mathrm{H}$ & -1.6159 & 3.3646 & -6.8748 & $\mathrm{H}$ & -9.1638 & -2.3878 & -0.8120 & C & 1.7158 & -5.4461 & -0.7324 \\
\hline $\mathrm{H}$ & -1.1326 & 4.8514 & -6.0306 & $\mathrm{H}$ & -7.7378 & 5.0080 & 3.0950 & C & 0.8023 & -5.9406 & 0.3962 \\
\hline $\mathrm{H}$ & -1.4533 & 1.0586 & -5.4787 & $\mathrm{H}$ & -9.4819 & 4.9034 & 3.0389 & C & 2.0092 & -3.9597 & -0.5337 \\
\hline $\mathrm{H}$ & -1.0797 & 1.1669 & -3.7516 & $\mathrm{H}$ & -7.5016 & 4.0082 & 1.1362 & $\mathrm{H}$ & 3.1946 & -4.9764 & -3.1947 \\
\hline $\mathrm{H}$ & 0.1758 & 1.5126 & -4.9454 & $\mathrm{H}$ & -7.9775 & 4.7545 & -0.3673 & $\mathrm{H}$ & 4.5192 & -4.7870 & -1.0241 \\
\hline $\mathrm{H}$ & -3.0450 & 5.3333 & -4.9475 & $\mathrm{H}$ & -9.6416 & 7.4232 & 3.2554 & $\mathrm{H}$ & 3.3234 & -6.4815 & 0.2549 \\
\hline $\mathrm{H}$ & 1.9181 & 3.6141 & -3.2118 & $\mathrm{H}$ & -8.8755 & 6.6491 & 4.6632 & $\mathrm{H}$ & 2.7549 & -7.2929 & -1.1988 \\
\hline $\mathrm{H}$ & 2.2485 & 4.1137 & -5.6067 & $\mathrm{H}$ & -7.8930 & 7.4954 & 3.4567 & $\mathrm{H}$ & 1.1787 & -5.5636 & -1.6862 \\
\hline $\mathrm{H}$ & 3.5519 & 2.5509 & -7.0447 & $\mathrm{H}$ & -10.9633 & -6.1232 & 1.7845 & $\mathrm{H}$ & 1.3100 & -5.8225 & 1.3606 \\
\hline $\mathrm{H}$ & 4.5444 & 0.5002 & -6.0409 & $\mathrm{H}$ & -10.2854 & -2.7148 & -2.1506 & $\mathrm{H}$ & -0.1174 & -5.3508 & 0.4397 \\
\hline $\mathrm{H}$ & 4.2197 & 0.0114 & -3.6526 & $\mathrm{H}$ & -8.9796 & -3.8600 & -1.7789 & $\mathrm{H}$ & 0.5398 & -6.9993 & 0.2833 \\
\hline C & -5.0442 & -3.3893 & 3.4187 & $\mathrm{H}$ & -11.6081 & -3.8461 & -0.6390 & $\mathrm{H}$ & 2.6184 & -3.5505 & -1.3413 \\
\hline C & -3.6198 & -2.8359 & 3.5886 & C & 3.9833 & 0.8262 & -1.0712 & $\mathrm{H}$ & 2.5440 & -3.8053 & 0.4100 \\
\hline 0 & -3.4191 & -1.6321 & 3.7368 & $\mathrm{H}$ & 4.1445 & -0.1934 & -1.4299 & $\mathrm{H}$ & 1.0812 & -3.3839 & -0.4875 \\
\hline C & -5.9270 & -2.4500 & 2.5755 & 0 & 3.6700 & 0.7261 & 0.3146 & C & 9.1431 & -5.4126 & -0.2780 \\
\hline C & -5.6442 & -2.6372 & 1.1051 & C & 5.2534 & 1.6653 & -1.2671 & C & 7.8963 & -4.5660 & -0.5857 \\
\hline $\mathrm{N}$ & -6.6041 & -3.1736 & 0.3416 & $\mathrm{H}$ & 6.0721 & 1.2069 & -0.7143 & C & 8.2476 & -3.2045 & -1.1396 \\
\hline 0 & -4.5319 & -2.3076 & 0.6230 & $\mathrm{H}$ & 5.5402 & 1.7291 & -2.3183 & C & 8.1399 & -2.9444 & -2.5126 \\
\hline $\mathrm{N}$ & -2.6145 & -3.7468 & 3.6902 & $\mathrm{H}$ & 5.0828 & 2.6766 & -0.8885 & C & 8.6971 & -2.1788 & -0.2990 \\
\hline C & -1.2624 & -3.3079 & 4.0169 & & & & & C & 8.4610 & -1.6865 & -3.0225 \\
\hline C & -0.5641 & -2.6551 & 2.8193 & En( & AA)-R2 & & & C & 9.0160 & -0.9161 & -0.8037 \\
\hline 0 & -0.9576 & -2.8392 & 1.6615 & & & & & C & 8.8963 & -0.6667 & -2.1716 \\
\hline C & -0.5408 & -4.6296 & 4.3513 & C & 11.2987 & 5.2653 & -1.0770 & $\mathrm{H}$ & 9.7916 & -4.9151 & 0.4510 \\
\hline C & -1.1960 & -5.6139 & 3.3708 & C & 9.8400 & 4.7904 & -1.1729 & $\mathrm{H}$ & 7.3104 & -4.4447 & 0.3349 \\
\hline C & -2.6680 & -5.1700 & 3.3287 & C & 9.3848 & 3.9413 & -0.0192 & $\mathrm{H}$ & 7.2665 & -5.0916 & -1.3084 \\
\hline $\mathrm{N}$ & 0.5453 & -1.9695 & 3.1479 & C & 8.6783 & 2.7585 & -0.0510 & $\mathrm{H}$ & 7.7674 & -3.7281 & -3.1651 \\
\hline C & 1.5490 & -1.6254 & 2.1700 & $\mathrm{~N}$ & 9.5826 & 4. 3291 & 1.2951 & $\mathrm{H}$ & 8.7893 & -2.3657 & 0.7678 \\
\hline C & 2.8761 & -2.3089 & 2.4996 & C & 8.9985 & 3.4003 & 2.0317 & $\mathrm{H}$ & 8.3617 & -1.5009 & -4.0883 \\
\hline 0 & 2.9547 & -3.5041 & 2.7785 & $\mathrm{~N}$ & 8.4382 & 2.4216 & 1.2676 & $\mathrm{H}$ & 9.3545 & -0.1312 & -0.1336 \\
\hline $\mathrm{N}$ & 3.9253 & -1.4587 & 2.4197 & $\mathrm{H}$ & 11.4556 & 5.7953 & -0.1341 & $\mathrm{H}$ & 9.1441 & 0.3125 & -2.5689 \\
\hline C & 5.3136 & -1.8151 & 2.6142 & $\mathrm{H}$ & 9.6829 & 4.2341 & -2.1053 & C & -2.7808 & 1.0009 & 0.4415 \\
\hline C & 5.8951 & -1.3540 & 3.9676 & $\mathrm{H}$ & 9.1911 & 5.6756 & -1.2340 & C & -1.6366 & 1.2496 & -0.2716 \\
\hline 0 & 7.1130 & -1.3997 & 4.1457 & $\mathrm{H}$ & 8.3257 & 2.1449 & -0.8637 & C & -1.6718 & 2.4212 & -1.1032 \\
\hline C & 6.1773 & -1.2144 & 1.5020 & $\mathrm{H}$ & 8.9495 & 3.3824 & 3.1110 & $\mathrm{~N}$ & -0.6089 & 2.8744 & -1.7371 \\
\hline 0 & 6.1113 & 0.2129 & 1.5161 & $\mathrm{H}$ & 7.8002 & 1.6891 & 1.5765 & $\mathrm{~N}$ & -2.8243 & 3.1215 & -1.2763 \\
\hline $\mathrm{N}$ & 5.0240 & -0.8928 & 4.8876 & C & -9.5729 & 1.7210 & -4.6021 & C & -3.9002 & 2.7932 & -0.5900 \\
\hline C & 5.5175 & -0.4350 & 6.1762 & $\mathrm{C}$ & -8.8239 & 2.5818 & -3.5728 & $\mathrm{~N}$ & -3.8910 & 1.7851 & 0.3117 \\
\hline $\mathrm{H}$ & -6.9842 & -2.6419 & 2.7801 & C & -7.7113 & 1.7970 & -2.9232 & $\mathrm{~S}$ & 1.6978 & -1.0624 & -3.2743 \\
\hline $\mathrm{H}$ & -5.6918 & -1.4086 & 2.8257 & $\mathrm{C}$ & -6.5396 & 1.5127 & -3.6407 & C & 1.3871 & 0.1292 & -1.9887 \\
\hline $\mathrm{H}$ & -5.0532 & -4.4028 & 3.0113 & C & -7.8398 & 1.2609 & -1.6377 & C & -5.1457 & 3.5707 & -0.8293 \\
\hline $\mathrm{H}$ & -0.7481 & -4.9091 & 5.3894 & C & -5.5434 & 0.7086 & -3.1050 & $\mathrm{~N}$ & 0.1151 & -0.1447 & -1.4413 \\
\hline $\mathrm{H}$ & 0.5399 & -4.5475 & 4.2184 & $\mathrm{C}$ & -6.8487 & 0.4522 & -1.0789 & C & -0.5042 & -1.3022 & -1.9674 \\
\hline $\mathrm{H}$ & -1.0883 & -6.6593 & 3.6705 & C & -5.6987 & 0.1666 & -1.8220 & C & -1.8275 & -1.7372 & -1.4426 \\
\hline $\mathrm{H}$ & -0.7495 & -5.4908 & 2.3808 & & & & & C & 0.1914 & -1.9175 & -2.9420 \\
\hline
\end{tabular}




\begin{tabular}{|c|c|c|c|}
\hline C & -0.1081 & -3.1673 & -3.7027 \\
\hline C & -0.3917 & 0.3903 & -0.1543 \\
\hline $\mathrm{H}$ & 0.3363 & 2.5728 & -1.4736 \\
\hline $\mathrm{H}$ & -0.6509 & 3.7276 & -2.2873 \\
\hline $\mathrm{H}$ & -0.6004 & -0.4520 & 0.5078 \\
\hline $\mathrm{H}$ & 0.4063 & 0.9675 & 0.2962 \\
\hline $\mathrm{H}$ & -5.6618 & 3.7512 & 0.1157 \\
\hline r & -4.8954 & 4.4994 & -1.3421 \\
\hline $\mathrm{H}$ & -5.8206 & 2.9911 & -1.4638 \\
\hline $\mathrm{H}$ & -2.8770 & 0.1684 & 1.1333 \\
\hline $\mathrm{H}$ & -1.7808 & -2.0003 & -0.3811 \\
\hline $\mathrm{H}$ & -2.1593 & -2.6157 & -1.9892 \\
\hline $\mathrm{H}$ & -2.5834 & -0.9656 & -1.5767 \\
\hline $\mathrm{H}$ & 0.6962 & -3.9032 & -3.5743 \\
\hline $\mathrm{H}$ & -0.2096 & -2.9789 & -4.7785 \\
\hline C & 1.7396 & -2.0575 & 7.6539 \\
\hline C & 0.9156 & -0.8052 & 7.3423 \\
\hline C & 1.7016 & 0.1334 & 6.4268 \\
\hline 0 & 1.9757 & -0.3400 & 5.2471 \\
\hline 0 & 2.0515 & 1.2512 & 6.8300 \\
\hline C & -8.4263 & 3.0953 & 3.7889 \\
\hline C & -7.5182 & 1.9071 & 3.4719 \\
\hline C & -6.6108 & 2.1611 & 2.2648 \\
\hline 0 & -6.6468 & 3.3013 & 1.7420 \\
\hline 0 & -5.8686 & 1.1912 & 1.8890 \\
\hline $\mathrm{H}$ & -9.0340 & 2.8965 & 4.6797 \\
\hline $\mathrm{H}$ & -7.8386 & 3.9965 & 3.9660 \\
\hline $\mathrm{H}$ & -8.0997 & 0.9974 & 3.2758 \\
\hline $\mathrm{H}$ & -6.8670 & 1.6624 & 4.3205 \\
\hline C & -0.9280 & 2.4917 & 5.2639 \\
\hline C & -0.7496 & 1.7696 & 3.9182 \\
\hline C & 0.5089 & 2.1217 & 3.1877 \\
\hline C & 0.7107 & 2.7541 & 1.9800 \\
\hline $\mathrm{N}$ & 1.7733 & 1.7900 & 3.6319 \\
\hline C & 2.6653 & 2.1887 & 2.6949 \\
\hline $\mathrm{N}$ & 2.0588 & 2.7759 & 1.6667 \\
\hline $\mathrm{H}$ & -0.0952 & 2.2773 & 5.9397 \\
\hline $\mathrm{H}$ & -0.7825 & 0.6858 & 4.0772 \\
\hline $\mathrm{H}$ & -1.5922 & 1.9979 & 3.2569 \\
\hline $\mathrm{H}$ & -0.0286 & 3.1756 & 1.3147 \\
\hline $\mathrm{H}$ & 3.7324 & 2.0614 & 2.7995 \\
\hline $\mathrm{H}$ & 2.1475 & 2.3077 & 0.0472 \\
\hline C & 5.3282 & 7.3922 & 2.2830 \\
\hline C & 5.2212 & 6.2009 & 3.2395 \\
\hline C & 5.9093 & 4.9138 & 2.7489 \\
\hline C & 5.8562 & 3.8395 & 3.8446 \\
\hline C & 5.2993 & 4.3912 & 1.4406 \\
\hline $\mathrm{H}$ & 4.8134 & 7.1979 & 1.3368 \\
\hline $\mathrm{H}$ & 4.1623 & 5.9806 & 3.4350 \\
\hline $\mathrm{H}$ & 5.6576 & 6.4805 & 4.2078 \\
\hline $\mathrm{H}$ & 6.9643 & 5.1498 & 2.5542 \\
\hline $\mathrm{H}$ & 4.8203 & 3.6146 & 4.1229 \\
\hline $\mathrm{H}$ & 6.3221 & 2.9029 & 3.5202 \\
\hline $\mathrm{H}$ & 6.3712 & 4.1722 & 4.7533 \\
\hline $\mathrm{H}$ & 4.2176 & 4.2463 & 1.5377 \\
\hline $\mathrm{H}$ & 5.7454 & 3.4314 & 1.1642 \\
\hline $\mathrm{H}$ & 5.4711 & 5.0836 & 0.6097 \\
\hline $\mathrm{H}$ & -1.0300 & -3.6329 & -3.3510 \\
\hline $\mathrm{H}$ & -9.0999 & 3.2937 & 2.9554 \\
\hline $\mathrm{H}$ & 11.9892 & 4.4157 & -1.0995 \\
\hline $\mathrm{H}$ & 11.5482 & 5.9359 & -1.9057 \\
\hline $\mathrm{H}$ & -10.3819 & 2.2808 & -5.0842 \\
\hline $\mathrm{H}$ & -8.8907 & 1.3689 & -5.3822 \\
\hline $\mathrm{H}$ & -0.9746 & 3.5756 & 5.1178 \\
\hline $\mathrm{H}$ & -1.8578 & 2.1688 & 5.7418 \\
\hline $\mathrm{H}$ & 6.3759 & 7.6155 & 2.0502 \\
\hline $\mathrm{H}$ & 4.8833 & 8.2922 & 2.7213 \\
\hline $\mathrm{H}$ & -0.0154 & -1.0925 & 6.8363 \\
\hline $\mathrm{H}$ & 0.6537 & -0.2657 & 8.2560 \\
\hline $\mathrm{H}$ & 2.0184 & -2.5632 & 6.7251 \\
\hline $\mathrm{H}$ & 1.1853 & -2.7620 & 8.2834 \\
\hline H & 2.6628 & -1.7913 & 8.1794 \\
\hline H & 8.8625 & -6.3908 & 0.1259 \\
\hline H & 9.7284 & -5.5753 & -1.1886 \\
\hline $\mathrm{H}$ & 5.0511 & -6.4107 & -1.4149 \\
\hline $\mathrm{H}$ & 0.2309 & -7.8967 & -4.9041 \\
\hline H & -0.7609 & -6.4573 & -5.2135 \\
\hline & 3.8899 & -5.1125 & -5.4588 \\
\hline & 1.9755 & 1.1265 & 4.4145 \\
\hline & -4.7720 & 1.5736 & 0.9384 \\
\hline & & & \\
\hline
\end{tabular}

\begin{tabular}{|c|c|c|c|}
\hline & 695 & 1279 & -3 \\
\hline & 11 & 257 & \\
\hline & -0.2503 & 435 & \\
\hline & -1 & & \\
\hline & -2.1659 & 9912 & \\
\hline & -1.9285 & & \\
\hline & -2.9049 & & \\
\hline & -3 & & \\
\hline & -2 & 39 & -4 \\
\hline & -1 & 2 & -5 \\
\hline & -0.7045 & 747 & \\
\hline & -0.9320 & 263 & \\
\hline & & $n \rho^{\prime}$ & \\
\hline & & & -1 \\
\hline & & & -1 \\
\hline & & & \\
\hline & & & -3 \\
\hline & 4.3084 & & \\
\hline & 2.2 & & -1 \\
\hline & 1.7 & 54 & -0 \\
\hline & & & -3 \\
\hline & & & -3 \\
\hline & -1.7 & & -1 \\
\hline & -2.2 & & -2 \\
\hline & -2.5 & & -3 \\
\hline & -3.2849 & 2. & -5 \\
\hline & -0 & & -4 \\
\hline & & & -6 \\
\hline & -1 & & -7 \\
\hline & -1 & & \\
\hline & -1.1667 & & \\
\hline & -0.9 & & -4 \\
\hline & 0.3 & & -5 \\
\hline & -3 & & -5 \\
\hline & & & -0 \\
\hline & 6.2 & & -1 \\
\hline & & & \\
\hline & & & \\
\hline & 3.8096 & -0.1815 & -3 \\
\hline & -5 & -2 & \\
\hline & -3 & -2 & \\
\hline & -3 & -1 & \\
\hline C & -6 & & \\
\hline$C$ & -5.6751 & -2 & \\
\hline & -6.6334 & -2.8262 & \\
\hline & -4 & -2.0267 & \\
\hline & -2 . & -3 & \\
\hline & -1 & -2 & \\
\hline - & -0 . & -2 & \\
\hline 0 & -0 . & -2 & \\
\hline$C$ & -0.5837 & -4.0088 & \\
\hline C & -1.2437 & -5 & \\
\hline 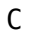 & -2 & -4 & \\
\hline & 2 & -1 & \\
\hline & & -1 & \\
\hline 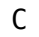 & & -1 . & \\
\hline 0 & & -2 & \\
\hline & & -0 . & \\
\hline & & -1 & \\
\hline 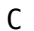 & & -0 & \\
\hline & & -0 & \\
\hline $\mathrm{C}$ & 6 & -0 . & \\
\hline 0 & & & \\
\hline & & & \\
\hline & & & \\
\hline & -7 & -2 & \\
\hline & -5 & -0 . & \\
\hline & -5 & -3 & \\
\hline & -0.8 & -4 & \\
\hline & & -3 & \\
\hline & -1 & -6 & \\
\hline & & & \\
\hline & -3 & -5 & \\
\hline & & & \\
\hline & -1.2854 & -1.92 & \\
\hline & חת & -0.1547 & \\
\hline & & -1.7435 & \\
\hline & & -0.88 & \\
\hline & & -1.44 & \\
\hline & & & \\
\hline & & & \\
\hline
\end{tabular}

\begin{tabular}{|c|c|c|c|}
\hline H & 6.0861 & 1.4470 & 年 \\
\hline $\mathrm{H}$ & .1518 & 6.5060 & \\
\hline & -4 . & 8 & \\
\hline & -2.9025 & 165 & -2 \\
\hline & -0.0788 & 122 & -2 \\
\hline & 594 & & \\
\hline & 846 & 1707 & \\
\hline & & & \\
\hline & 686 & -1.2 & \\
\hline & -7 & -3 & \\
\hline & -6 . & -3 & -0 . \\
\hline & 3. & & \\
\hline & -5. & -2 . & \\
\hline 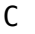 & -11.0909 & -4.95 & \\
\hline $\mathrm{C}$ & -10. & -4. & \\
\hline 0 & 107 & -4.23 & \\
\hline & -11. & -3 & \\
\hline & -10. & -4 & \\
\hline C & -9.7259 & -3.40 & -0 \\
\hline C & -9. & & \\
\hline C & -8.8783 & 5. & \\
\hline C & -8 & & \\
\hline & -8 . & & \\
\hline 0 & -9.35 & 6.7 & -0 \\
\hline $\mathrm{H}$ & -10.3 & -3 & \\
\hline $\mathrm{H}$ & -11.9506 & -4.28 & \\
\hline & -11.6867 & -2.98 & \\
\hline $\mathrm{H}$ & -12 . & -5 & \\
\hline 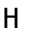 & -9.3070 & -2.4680 & -0 \\
\hline $\mathrm{H}$ & -7 & 5. & \\
\hline $\mathrm{H}$ & -9 & 5. & \\
\hline $\mathrm{H}$ & -7 & 4. & \\
\hline $\mathrm{H}$ & -8 . & 4. & -1 . \\
\hline $\mathrm{H}$ & -9 . & 7.74 & \\
\hline $\mathrm{H}$ & -9 . & 7 & \\
\hline $\mathrm{H}$ & -8 & 7. & \\
\hline r & -10 & -5 & \\
\hline $\mathrm{H}$ & -10 . & & \\
\hline $\mathrm{H}$ & -8. & -4 & -1 . \\
\hline $\mathrm{H}$ & -11. & -4 & \\
\hline 0 & 5. & -1.8229 & -1 \\
\hline $\mathrm{H}$ & & -1 . & -1 . \\
\hline 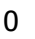 & & -2 . & -2 \\
\hline C & 4. & -1.4 & -0 \\
\hline $\mathrm{H}$ & & -2 . & \\
\hline $\mathrm{H}$ & & -0 . & -0 \\
\hline & & -1 . & 1 \\
\hline
\end{tabular}

Int1(AA)-S

$\begin{array}{rrrr}\mathrm{C} & 11.4703 & 5.3987 & -0.6980 \\ \mathrm{C} & 9.9343 & 5.3864 & -0.5942 \\ \mathrm{C} & 9.3751 & 4.2550 & 0.2226 \\ \mathrm{C} & 8.4172 & 3.3275 & -0.1279 \\ \mathrm{~N} & 9.7650 & 4.0474 & 1.5342 \\ \mathrm{C} & 9.0505 & 3.0174 & 1.9549 \\ \mathrm{~N} & 8.2205 & 2.5392 & 0.9875 \\ \mathrm{H} & 11.9103 & 5.4081 & 0.3027 \\ \mathrm{H} & 9.4915 & 5.3513 & -1.5972 \\ \mathrm{H} & 9.6096 & 6.3369 & -0.1487 \\ \mathrm{H} & 7.8636 & 3.1712 & -1.0410 \\ \mathrm{H} & 9.0847 & 2.5804 & 2.9430 \\ \mathrm{H} & 7.5302 & 1.7895 & 1.1019 \\ \mathrm{C} & -9.3506 & 1.9680 & -4.6131 \\ \mathrm{C} & -8.5190 & 2.7522 & -3.5813 \\ \mathrm{C} & -7.4286 & 1.9164 & -2.9470 \\ \mathrm{C} & -6.2755 & 1.5792 & -3.6723 \\ \mathrm{C} & -7.5404 & 1.4272 & -1.6399 \\ \mathrm{C} & -5.2817 & 0.7768 & -3.1232 \\ \mathrm{C} & -6.5497 & 0.6267 & -1.0670 \\ \mathrm{C} & -5.4139 & 0.2975 & -1.8140 \\ \mathrm{O} & -4.3866 & -0.4388 & -1.3190 \\ \mathrm{H} & -9.8392 & 1.1085 & -4.1426 \\ \mathrm{H} & -9.1798 & 3.1440 & -2.7996 \\ \mathrm{H} & -8.0737 & 3.6249 & -4.0759 \\ \mathrm{H} & -6.1537 & 1.9520 & -4.6867 \\ \mathrm{H} & -8.4039 & 1.7054 & -1.0409 \\ \mathrm{H} & -4.3929 & 0.5144 & -3.6862 \\ \mathrm{H} & -6.6131 & 0.3286 & -0.0253 \\ \mathrm{H} & -4.6118 & -0.8686 & -0.4703\end{array}$




\begin{tabular}{|c|c|c|c|}
\hline C & -0.1250 & -6.8459 & -4.7059 \\
\hline C & -1.2765 & -7.4483 & -3.8808 \\
\hline C & -2.2218 & -6.4117 & -3.3111 \\
\hline C & -2.2485 & -6.1150 & -1.9437 \\
\hline C & -3.0946 & -5.6985 & -4.1458 \\
\hline C & -3.0902 & -5.1311 & -1.4205 \\
\hline C & -3.9454 & -4.7192 & -3.6428 \\
\hline C & -3.9424 & -4.4208 & 2740 \\
\hline 0 & -4.7919 & -3.4517 & -1.8336 \\
\hline $\mathrm{H}$ & 0.4939 & -6.1890 & -4.0858 \\
\hline $\mathrm{H}$ & -1.8381 & -8.1467 & -4.5146 \\
\hline $\mathrm{H}$ & -0.8584 & -8.0434 & -3.0615 \\
\hline $\mathrm{H}$ & -1.5948 & -6.6576 & -1.2685 \\
\hline $\mathrm{H}$ & -3.1051 & -5.9117 & -5.2122 \\
\hline $\mathrm{H}$ & -3.0830 & -4.9085 & -0.3574 \\
\hline $\mathrm{H}$ & -4.6135 & -4.1634 & -4.2924 \\
\hline $\mathrm{H}$ & -4.4856 & -3.0576 & -0.9784 \\
\hline C & 5.0970 & -4.4453 & -5.2025 \\
\hline C & 5.1883 & -5.2790 & -3.9515 \\
\hline 0 & 5.8558 & -6.3126 & -3.9272 \\
\hline C & 4.4363 & -3.0694 & -5.1044 \\
\hline $\mathrm{H}$ & 6.1206 & -4.3422 & -5.5743 \\
\hline $\mathrm{H}$ & 4.9599 & -2.4394 & -4.3811 \\
\hline $\mathrm{H}$ & 3.3819 & -3.1357 & -4.8135 \\
\hline $\mathrm{H}$ & 4.4674 & -2.5565 & -6.0697 \\
\hline $\mathrm{N}$ & 4.4600 & -4.8301 & -2.9009 \\
\hline C & 4.4121 & -5.5732 & -1.6717 \\
\hline C & 2.9872 & -5.9906 & -1.2678 \\
\hline C & 2.2518 & -4.9835 & -0.3664 \\
\hline C & 0.7546 & -5.2949 & -0.3011 \\
\hline C & 2.8589 & -4.9624 & 1.0411 \\
\hline $\mathrm{H}$ & 4.0068 & -3.9327 & -2.9513 \\
\hline $\mathrm{H}$ & 4.8767 & -4.9890 & -0.8681 \\
\hline $\mathrm{H}$ & 3.0318 & -6.9503 & -0.7359 \\
\hline $\mathrm{H}$ & 2.4141 & -6.1740 & -2.1860 \\
\hline $\mathrm{H}$ & 2.3602 & -3.9829 & -0.8101 \\
\hline $\mathrm{H}$ & 0.5881 & -6.3030 & 0.1019 \\
\hline $\mathrm{H}$ & 0.2271 & -4.5876 & 0.3457 \\
\hline $\mathrm{H}$ & 0.3050 & -5.2639 & -1.2984 \\
\hline $\mathrm{H}$ & 3.8916 & -4.6011 & 1.0515 \\
\hline $\mathrm{H}$ & 2.8602 & -5.9741 & 1.4650 \\
\hline $\mathrm{H}$ & 2.2927 & -4.3295 & 1.7250 \\
\hline C & 9.3304 & -5.3075 & -0.3763 \\
\hline C & 7.9802 & -5.2003 & -1.1067 \\
\hline C & 7.7645 & -3.8531 & -1.7611 \\
\hline C & 7.8564 & -3.6914 & -3.1482 \\
\hline C & 7.4864 & -2.7246 & -0.9800 \\
\hline C & 7.6604 & -2.4395 & -3.7353 \\
\hline C & 7.2848 & -1.4711 & -1.5581 \\
\hline C & 7.3665 & -1.3266 & -2.9452 \\
\hline $\mathrm{H}$ & 9.4157 & -4.5458 & 0.4048 \\
\hline $\mathrm{H}$ & 7.1757 & -5.3794 & -0.3834 \\
\hline $\mathrm{H}$ & 7.8911 & -5.9852 & -1.8645 \\
\hline $\mathrm{H}$ & 8.0575 & -4.5594 & -3.7685 \\
\hline $\mathrm{H}$ & 7.4305 & -2.8341 & 0.0983 \\
\hline $\mathrm{H}$ & 7.7355 & -2.3342 & -4.8143 \\
\hline $\mathrm{H}$ & 7.0728 & -0.6112 & -0.9297 \\
\hline $\mathrm{H}$ & 7.2091 & -0.3539 & -3.4015 \\
\hline C & -2.3811 & 1.0106 & 0.7328 \\
\hline C & -1.2114 & 1.2446 & 0.0509 \\
\hline C & -1.1992 & 2.4048 & -0.7961 \\
\hline $\mathrm{N}$ & -0.0956 & 2.8448 & -1.3729 \\
\hline $\mathrm{N}$ & -2.3443 & 3.1011 & -1.0315 \\
\hline C & -3.4439 & 2.7925 & -0.3696 \\
\hline $\mathrm{N}$ & -3.4760 & 1.8015 & 0.5521 \\
\hline S & 1.3461 & -1.0999 & -3.2491 \\
\hline C & 1.4235 & -0.0909 & -1.8593 \\
\hline C & -4.6838 & 3.5695 & -0.6516 \\
\hline $\mathrm{N}$ & 0.3882 & -0.3530 & -1.0379 \\
\hline C & -0.4489 & -1.4085 & -1.4618 \\
\hline C & -1.5991 & -1.8861 & -0.6504 \\
\hline C & -0.0621 & -1.9245 & -2.6599 \\
\hline C & -0.7013 & -3.0320 & -3.4395 \\
\hline C & 0.0298 & 0.3981 & 0.2190 \\
\hline $\mathrm{H}$ & 0.8400 & 2.5085 & -1.0946 \\
\hline $\mathrm{H}$ & -0.1100 & 3.7052 & -1.9199 \\
\hline $\mathrm{H}$ & -0.1310 & -0.3466 & 0.9950 \\
\hline $\mathrm{H}$ & 0.8906 & 1.0006 & 0.4929 \\
\hline $\mathrm{H}$ & -5.2906 & 3.6618 & 0.2534 \\
\hline $\mathrm{H}$ & -4.4141 & 4.5486 & -1.0485 \\
\hline $\mathrm{H}$ & -5.2753 & 3.0435 & -1.406 \\
\hline
\end{tabular}

\begin{tabular}{|c|c|c|c|}
\hline $\mathrm{H}$ & -2.5014 & 0.2047 & 1.4535 \\
\hline $\mathrm{H}$ & -1.3222 & -2.0980 & 0.3860 \\
\hline $\mathrm{H}$ & -1.9539 & -2.8213 & -1.0808 \\
\hline $\mathrm{H}$ & -2.4216 & -1.1755 & -0.6827 \\
\hline $\mathrm{H}$ & -0.1282 & -3.2652 & -4.3398 \\
\hline $\mathrm{H}$ & -1.7179 & -2.7681 & -3.7428 \\
\hline C & 1.8279 & -2.3044 & 7.6034 \\
\hline C & 0.9849 & -1.0431 & 7.3738 \\
\hline C & 1.7482 & -0.0103 & 6.5309 \\
\hline 0 & 1.9058 & -0.3313 & 5.2817 \\
\hline 0 & 2.1800 & 1.0182 & 7.0628 \\
\hline C & -8.3046 & 2.9884 & 3.8413 \\
\hline C & -7.3333 & 1.8570 & 3.4867 \\
\hline C & -6.3596 & 2.1963 & 2.3493 \\
\hline 0 & -6.3748 & 3.3598 & 1.8824 \\
\hline 0 & -5.5815 & 1.2524 & 1.9702 \\
\hline $\mathrm{H}$ & -8.9432 & 2.6997 & 4.6837 \\
\hline $\mathrm{H}$ & -7.7631 & 3.8952 & 4.1130 \\
\hline II & -7.8728 & 0.9443 & 3.2033 \\
\hline $\mathrm{H}$ & -6.7217 & 1.5755 & 4.3530 \\
\hline C & -0.8225 & 2.3368 & 5.3769 \\
\hline C & -0.8234 & 1.7722 & 3.9475 \\
\hline C & 0.3978 & 2.1654 & 3.1830 \\
\hline C & 0.6048 & 3.0513 & 2.1454 \\
\hline iv & 1.6520 & 1.6765 & 3.4899 \\
\hline C & 2.5388 & 2.2677 & 2.6478 \\
\hline IN & 1.9445 & 3.1056 & 1.8085 \\
\hline $\mathrm{H}$ & 0.0658 & 2.0239 & 318 \\
\hline $\mathrm{H}$ & -0.9142 & 0.6791 & 3.9779 \\
\hline $\mathrm{H}$ & -1.7048 & 2.1359 & 3.4073 \\
\hline $\mathrm{H}$ & -0.1318 & 3.6589 & 1.6374 \\
\hline $\mathrm{H}$ & 3.5931 & 2.0422 & 2.6670 \\
\hline $\mathrm{H}$ & 2.8770 & 1.7322 & -0.0404 \\
\hline C & 5.4564 & 7.3706 & 2.6790 \\
\hline C & 5.3546 & 6.0428 & 3.4378 \\
\hline C & 5.9019 & 4.8090 & 2.6934 \\
\hline C & 5.8466 & 3.5840 & 3.6187 \\
\hline C & 5.1501 & 4.5437 & 1.3812 \\
\hline $\mathrm{H}$ & 4.8519 & 7.3607 & 1.7664 \\
\hline $\mathrm{H}$ & 4.3036 & 5.8519 & 3.6959 \\
\hline $\mathrm{H}$ & 5.8897 & 6.1379 & 4. 3924 \\
\hline $\mathrm{H}$ & 6.9567 & 4. 9981 & 2.4469 \\
\hline $\mathrm{H}$ & 4.8287 & 3.4217 & 3.9900 \\
\hline $\mathrm{H}$ & 6.1619 & 2.6704 & 3.1066 \\
\hline $\mathrm{H}$ & 6.4941 & 3.7199 & 4.4924 \\
\hline $\mathrm{H}$ & 4.0742 & 4.4199 & 1.5459 \\
\hline $\mathrm{H}$ & 5.5200 & 3.6291 & 0.9078 \\
\hline $\mathrm{H}$ & 5.2935 & 5.3587 & 0.6632 \\
\hline $\mathrm{H}$ & -0.7697 & -3.9424 & -2.8402 \\
\hline $\mathrm{H}$ & -8.9466 & 3.2232 & 2.9919 \\
\hline $\mathrm{H}$ & 11.8343 & 4.5034 & -1.2131 \\
\hline $\mathrm{H}$ & 11.8220 & 6.2796 & -1.2460 \\
\hline $\mathrm{H}$ & -10.1239 & 2.6004 & -5.0614 \\
\hline $\mathrm{H}$ & -8.7135 & 1.5862 & -5.4173 \\
\hline $\mathrm{H}$ & -0.8275 & 3.4311 & 5.3517 \\
\hline $\mathrm{H}$ & -1.7093 & 2.0031 & 5.9268 \\
\hline $\mathrm{H}$ & 6.4927 & 7.5777 & 2.3865 \\
\hline $\mathrm{H}$ & 5.1093 & 8.2065 & 3.2964 \\
\hline $\mathrm{H}$ & 0.0585 & -1.3099 & 6.8491 \\
\hline $\mathrm{H}$ & 0.7127 & -0.5800 & 8.3263 \\
\hline $\mathrm{H}$ & 2.1681 & -2.7122 & 6.6465 \\
\hline $\mathrm{H}$ & 1.2642 & -3.0781 & 8.1366 \\
\hline $\mathrm{H}$ & 2.7179 & -2.0661 & 8.1951 \\
\hline $\mathrm{H}$ & 9.4432 & -6.2897 & 0.0937 \\
\hline $\mathrm{H}$ & 10.1631 & -5.1629 & -1.0722 \\
\hline $\mathrm{H}$ & 5.0404 & -6.4482 & -1.8383 \\
\hline $\mathrm{H}$ & 0.5191 & -7.6300 & -5.1160 \\
\hline $\mathrm{H}$ & -0.5096 & -6.2500 & -5.5401 \\
\hline $\mathrm{H}$ & 4.5772 & -5.0637 & -5.9451 \\
\hline $\mathrm{H}$ & 1.8642 & 0.9842 & 4.2443 \\
\hline $\mathrm{H}$ & -4.4010 & 1.6095 & 1.1368 \\
\hline C & 2.2300 & 6.8470 & -3.1616 \\
\hline C & 0.9310 & 6.2049 & -2.7514 \\
\hline 0 & 0.7367 & 4.9955 & -2.9161 \\
\hline $\mathrm{N}$ & -0.0099 & 7.0105 & -2.2029 \\
\hline C & -1.3657 & 6.5136 & -2.0475 \\
\hline C & -1.9369 & 6.0947 & -3.4145 \\
\hline 0 & -1.7231 & 6.7792 & -4.4124 \\
\hline $\mathrm{N}$ & -2.6802 & 4.9675 & -3.3811 \\
\hline C & -3.1955 & 4.3539 & -4.5952 \\
\hline C & -2.6 & 2.9471 & -4 \\
\hline
\end{tabular}

\begin{tabular}{|c|c|c|c|}
\hline$C$ & -1.0928 & 2.8705 & -4 \\
\hline & -0.6435 & 1.4077 & \\
\hline & -0.5472 & 3.6785 & \\
\hline & 3.1888 & 932 & \\
\hline & 2.7748 & 081 & \\
\hline 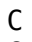 & & & \\
\hline C & 912 & 692 & \\
\hline & & & \\
\hline & & a & \\
\hline & & 0.7848 & \\
\hline & 2.7 & 1.9179 & \\
\hline & & & \\
\hline & 3. & & \\
\hline & -1.3 & & \\
\hline $\mathrm{H}$ & -1 . & & \\
\hline & -2 & 78 & \\
\hline 11 & -3 & 205 & \\
\hline & -0 . & 075 & \\
\hline 1 & 0. & 50 & \\
\hline & -0 . & & \\
\hline II & -0 . & & \\
\hline 11 & -1 . & & \\
\hline & -1 & & \\
\hline 11 & 3 & 39 & \\
\hline n & -2 . & 29 & -5 \\
\hline | & 2. & & \\
\hline & & & \\
\hline & 4.4 & & \\
\hline $\mathrm{H}$ & 5.2 & & \\
\hline П & 4. & & \\
\hline C & -4.9729 & -2.9559 & \\
\hline C & -3.5348 & -2.3977 & \\
\hline 0 & -3 & -1 & \\
\hline C & -5 & -2 & \\
\hline C & -5 & & \\
\hline & -6. & -3 & \\
\hline 0 & -4 & -2 & \\
\hline & -2 . & -3 & \\
\hline C & -1. & -2.7 & \\
\hline C & -0. & -2. & \\
\hline 0 & -0 . & -3 & \\
\hline C & -0 & -3 & \\
\hline C & -1. & -5 & \\
\hline C & -2. & -4 & \\
\hline & & -1.7 & \\
\hline C & 1. & -1.6 & \\
\hline C & 3. & -1 & \\
\hline 0 & 3.2 & & \\
\hline IV & 4. & -1 & \\
\hline C & 5. & -1 & \\
\hline C & 5.96 & -0 & \\
\hline 0 & & & \\
\hline C & 6. & -0. & \\
\hline 0 & 6. & & \\
\hline & 5. & -0. & \\
\hline C & 5.2 & & \\
\hline H & -6. & -2.3 & \\
\hline & -5 & -1 & \\
\hline & -4 & $-4 . e$ & \\
\hline F & -0 . & -3.7 & \\
\hline $\mathrm{H}$ & 0. & -3.8 & \\
\hline & -1 & -5 & \\
\hline & -0 . & -5 & \\
\hline & -3 & -4 & \\
\hline & -3. & -5 & \\
\hline$r$ & -1.2 & -1.7 & \\
\hline & 1. & -0.5 & \\
\hline & 1. & -2 & \\
\hline & 7. & -0. & \\
\hline $\mathrm{H}$ & 6.1 & -1 & \\
\hline $\mathrm{H}$ & 5.2 & 0. & \\
\hline $\mathrm{H}$ & 5. & -2.3681 & \\
\hline $\mathrm{H}$ & 6.2 & 1.2 & \\
\hline & & & \\
\hline $\mathrm{H}$ & -4.2 & 4.2 & \\
\hline $\mathrm{H}$ & -2.6158 & 4.3770 & \\
\hline $\mathrm{H}$ & & 8. & \\
\hline $\mathrm{H}$ & & -0.2909 & \\
\hline $\mathrm{H}$ & 4. & & \\
\hline & 4. & -0. & \\
\hline & 0. & & \\
\hline
\end{tabular}




$\begin{array}{rrrr}\text { H } & -7.3279 & -3.8149 & 1.0437 \\ \text { H } & -6.2582 & -3.7861 & -0.3235 \\ \text { H } & 3.8230 & -0.4149 & 1.9354 \\ \text { H } & -5.3868 & -2.7905 & 4.6642 \\ \mathrm{C} & -11.2483 & -4.8807 & 1.8122 \\ \mathrm{C} & -10.1379 & -4.4892 & 0.8610 \\ 0 & -8.9557 & -4.6903 & 1.1552 \\ \mathrm{C} & -11.1296 & -4.0020 & 3.0609 \\ \mathrm{~N} & -10.5098 & -3.8504 & -0.2699 \\ \mathrm{C} & -9.5338 & -3.3028 & -1.1944 \\ \mathrm{C} & -8.8898 & 7.1779 & 2.7492 \\ \mathrm{C} & -8.6910 & 5.8261 & 2.0789 \\ \mathrm{C} & -8.6325 & 5.8758 & 0.5545 \\ \mathrm{~N} & -8.0942 & 4.7484 & -0.0007 \\ \mathrm{O} & -9.0786 & 6.8071 & -0.1087 \\ \mathrm{H} & -10.1357 & -4.1150 & 3.4995 \\ \mathrm{H} & -11.8754 & -4.2782 & 3.8114 \\ \mathrm{H} & -11.2743 & -2.9459 & 2.8123 \\ \mathrm{H} & -12.2363 & -4.7781 & 1.3498 \\ \mathrm{H} & -8.9321 & -2.5184 & -0.7249 \\ \mathrm{H} & -7.7915 & 5.3242 & 2.4421 \\ \mathrm{H} & -9.5275 & 5.1645 & 2.3353 \\ \mathrm{H} & -7.5287 & 4.1236 & 0.5736 \\ \mathrm{H} & -7.9380 & 4.7622 & -0.9978 \\ \mathrm{H} & -9.7658 & 7.6871 & 2.3390 \\ \mathrm{H} & -9.0225 & 7.0574 & 3.8287 \\ \mathrm{H} & -8.0278 & 7.8318 & 2.5839 \\ \mathrm{H} & -11.1088 & -5.9333 & 2.0756 \\ \mathrm{H} & -10.0552 & -2.8807 & -2.0541 \\ \mathrm{H} & -8.8553 & -4.0865 & -1.5423 \\ \mathrm{H} & -11.4899 & -3.6873 & -0.4362 \\ \mathrm{C} & 3.7963 & -0.0097 & -0.7756 \\ 0 & 3.7289 & 0.5388 & 0.4728 \\ \mathrm{C} & 3.6475 & -1.5324 & -0.7895 \\ \mathrm{H} & 2.6841 & -1.8390 & -0.3755 \\ \mathrm{H} & 3.7458 & -1.9536 & -1.7946 \\ \mathrm{H} & 4.4273 & -1.9798 & -0.1724 \\ \mathrm{H} & 4.7402 & 0.2496 & -1.2853\end{array}$

\section{Int1(AA)-R}

$\begin{array}{rrrr}\mathrm{C} & 11.5049 & 5.0792 & -0.2949 \\ \mathrm{C} & 9.9683 & 5.0465 & -0.2002 \\ \mathrm{C} & 9.4002 & 3.8283 & 0.4767 \\ \mathrm{C} & 8.3493 & 3.0285 & 0.0751 \\ \mathrm{~N} & 9.8596 & 3.4049 & 1.7113 \\ \mathrm{C} & 9.0951 & 2.3753 & 2.0375 \\ \mathrm{~N} & 8.1672 & 2.1009 & 1.0810 \\ \mathrm{H} & 11.9406 & 4.9672 & 0.7012 \\ \mathrm{H} & 9.5284 & 5.1316 & -1.2015 \\ \mathrm{H} & 9.6400 & 5.9382 & 0.3526 \\ \mathrm{H} & 7.7168 & 3.0475 & -0.7991 \\ \mathrm{H} & 9.1632 & 1.7970 & 2.9482 \\ \mathrm{H} & 7.4203 & 1.3978 & 1.1466 \\ \mathrm{C} & -9.4702 & 2.6545 & -4.1401 \\ \mathrm{C} & -8.6193 & 3.2673 & -3.0122 \\ \mathrm{C} & -7.5263 & 2.3410 & -2.5251 \\ \mathrm{C} & -6.3883 & 2.1044 & -3.3115 \\ \mathrm{C} & -7.6175 & 1.6742 & -1.2973 \\ \mathrm{C} & -5.3893 & 1.2304 & -2.8979 \\ \mathrm{C} & -6.6210 & 0.7987 & -0.8598 \\ \mathrm{C} & -5.5003 & 0.5738 & -1.6661 \\ \mathrm{O} & -4.4671 & -0.2262 & -1.2979 \\ \mathrm{H} & -9.9581 & 1.7351 & -3.8000 \\ \mathrm{H} & -9.2679 & 3.5393 & -2.1716 \\ \mathrm{H} & -8.1745 & 4.2030 & -3.3743 \\ \mathrm{H} & -6.2816 & 2.6162 & -4.2651 \\ \mathrm{H} & -8.4685 & 1.8692 & -0.6495 \\ \mathrm{H} & -4.5106 & 1.0493 & -3.5067 \\ \mathrm{H} & -6.6666 & 0.3585 & 0.1312 \\ \mathrm{H} & -4.6737 & -0.7635 & -0.5077 \\ \mathrm{C} & -0.4537 & -6.2339 & -5.7228 \\ \mathrm{C} & -1.4387 & -6.9170 & -4.7576 \\ \mathrm{C} & -2.3628 & -5.9482 & -4.0500 \\ \mathrm{C} & -2.3020 & -5.7518 & -2.6670 \\ \mathrm{C} & -3.3059 & -5.1998 & -4.7697 \\ \mathrm{C} & -3.1309 & -4.8381 & -2.0123 \\ \mathrm{C} & -4.1383 & -4.2823 & -4.1390 \\ \mathrm{C} & -4.0520 & -4.0872 & -2.7532 \\ 0 & -4.8855 & -3.1698 & -2.1970 \\ & & & \end{array}$

\begin{tabular}{|c|c|c|c|}
\hline & 0.1935 & -5.5353 & -5.1805 \\
\hline & -2.0332 & -7.6492 & -5.3198 \\
\hline $\mathrm{H}$ & -0.8754 & -7.4888 & -4.0115 \\
\hline & -1.5840 & -6.3175 & -2.0821 \\
\hline & -3.3866 & -5.3338 & -5.8458 \\
\hline & -3.0523 & -4.6952 & -0.9384 \\
\hline & -4.8584 & -3.6950 & -4.6993 \\
\hline & -4.5775 & -2.8801 & -1 \\
\hline & 4.8100 & -3.8895 & -6.0009 \\
\hline & 5.0997 & -4.4833 & -4.6498 \\
\hline & 6.2364 & -4.5121 & -4.1851 \\
\hline & 4.9165 & -2.3564 & -5.9370 \\
\hline & 5.5508 & -4.2821 & -6.7035 \\
\hline & 5.9010 & -2.0665 & -5.5615 \\
\hline & 4.1596 & -1.9454 & -5 \\
\hline & 4.7717 & -1.9080 & -6 \\
\hline & 4.0136 & -4.9116 & -3.9612 \\
\hline & 4.1765 & -5.4997 & -2.6521 \\
\hline & 2.8567 & -5.6641 & -1 \\
\hline & 2.0829 & -4.3654 & -1.6367 \\
\hline & 0.8637 & -4.6837 & -0.7682 \\
\hline & 2.9665 & -3.3018 & -0.9804 \\
\hline & 3.1 & -4.9863 & -4 \\
\hline & 4.8602 & -4.8 & -2.0839 \\
\hline & 3.0791 & -6.1491 & -0.9529 \\
\hline & 2.2084 & -6.3627 & -2.4614 \\
\hline & 1. & -3 & -2 \\
\hline & 1.1743 & -4.9918 & 0.2357 \\
\hline & 0.2038 & -3.8263 & -0.6373 \\
\hline & 0.2 & -5 & -1 \\
\hline & 3.7877 & -2 & -1.6281 \\
\hline & 3.4048 & -3.6791 & -0.0490 \\
\hline & 2.3913 & -2.4 & -0.7227 \\
\hline & 9.1 & -5 & -1 . \\
\hline & 7.9227 & -4 . & -1 \\
\hline & 8.2423 & -3.1595 & -1.1154 \\
\hline - & 7.7878 & -2 & -2.1636 \\
\hline & & & 26 \\
\hline & 8.0931 & -0. & -2 \\
\hline & 9.3179 & -1.2124 & -0.1299 \\
\hline & 8.8579 & -0.2 & -1.1770 \\
\hline & 9.9 & & \\
\hline & 7.5648 & -4.9115 & -0.0852 \\
\hline & 7.1166 & -4.8441 & -1.7934 \\
\hline & 7.1980 & -2 & -2.9564 \\
\hline & 9.3 & -3 & 94 \\
\hline$\pi$ & 7.7245 & -0.3692 & -3.0073 \\
\hline & 9.8918 & -0.7676 & 0.6773 \\
\hline & & & -1.1 \\
\hline & -2.36 & 0. & 0. \\
\hline & -1.2134 & 1.2562 & 0.1747 \\
\hline C & -1.2265 & 2.5211 & -0.5022 \\
\hline & -0. & & -1 \\
\hline & -2.3 & 3.2 & -0.5926 \\
\hline C & -3.4511 & 2.8540 & 0.0518 \\
\hline & -3.4609 & 1.7 & 0.8192 \\
\hline & & -0 . & -3 \\
\hline & 1.4 & & -1 \\
\hline$c$ & -4.6929 & 3.6681 & -0.0734 \\
\hline N & 0.3752 & -0.2 & -1.1438 \\
\hline & -0.2 & 4 & -1.6430 \\
\hline & -1.6 & -1.7 & -0.9 \\
\hline C & -0.0642 & -1.67 & -2.8578 \\
\hline$C$ & -0.69 & -2 & -3.7138 \\
\hline & 0 & .40 & 0.1960 \\
\hline$\pi$ & 0.7 & 2. & -0.8766 \\
\hline $\mathrm{H}$ & -0.1813 & 3.9 & -1.4953 \\
\hline & -0.10 & -0.4 & \\
\hline & 0.96 & 0.5 & 0.5310 \\
\hline $\mathrm{H}$ & -5.2513 & 3.6 & 0.8669 \\
\hline & -4.4295 & 4.68 & -0.3703 \\
\hline & & & -0.8483 \\
\hline & -2.465 & 0.03 & 1.459 \\
\hline & -1.432 & -2.0270 & 0.1 \\
\hline & -2.033 & -2.5959 & -1.4215 \\
\hline & & 722 & -0.9060 \\
\hline & -0.6434 & -3.7105 & -3.2349 \\
\hline & -0.1949 & & -4.6830 \\
\hline & 1.8717 & -3.5456 & 7.0571 \\
\hline & 1. & & \\
\hline & 1.8303 & & דצנת. \\
\hline
\end{tabular}

\begin{tabular}{|c|c|c|c|}
\hline & 1.9465 & -1.2465 & 5.1089 \\
\hline & 2.2806 & -0.1483 & 7.0460 \\
\hline & -8.2169 & 2.4302 & 3450 \\
\hline & -7.2631 & 1.3604 & (1) \\
\hline & -6.3010 & 1.8656 & 7159 \\
\hline & -6.3046 & 3.0890 & 2.4421 \\
\hline & -5.5427 & 0.9851 & 2.1781 \\
\hline & -8.8406 & 2.0191 & 5.146 \\
\hline & -7.6609 & 3.2808 & 4.7409 \\
\hline & -7.8168 & 0.5084 & 3.3857 \\
\hline & -6.6 & 0.9405 & 4.6016 \\
\hline & -0.7194 & 1.4183 & 5.5842 \\
\hline & -0.7502 & 1.1184 & 4.079 \\
\hline & 0.4904 & & 3.3787 \\
\hline & 0.7394 & 37 & \\
\hline & 1.7153 & 0.9 & 3.5860 \\
\hline & 2.6264 & 1.6093 & 2.8139 \\
\hline & 2.0742 & & \\
\hline & 0.1 & 0.5 & \\
\hline & -0.9031 & 0.0434 & \\
\hline & -1.6094 & 1.6203 & \\
\hline & $0 . e$ & & \\
\hline & 3.6 & & \\
\hline & 2.7793 & 1.6965 & 0.0235 \\
\hline & 5.6129 & 6.6632 & 3.4784 \\
\hline & 5.4 & & 4. \\
\hline & 6.0144 & 4.7 & 3. \\
\hline & 5.9301 & 2.7 & 3. \\
\hline & 5.2 & & 1.8660 \\
\hline & 4.9 & & \\
\hline & 4.4 & 5. & 4. \\
\hline & 6.0 & 5. & 70 \\
\hline & & & \\
\hline & 4. & & \\
\hline & 6.2269 & 1. & \\
\hline & 6.5719 & 2.8 & \\
\hline & & & \\
\hline & 5.5 & & \\
\hline & 5.4 & & \\
\hline & -1.7504 & -2.5 & -3.8946 \\
\hline & -8.8 & & \\
\hline & $11 . \varepsilon$ & 7 & -0 . \\
\hline & 11.8511 & 6.6 & -0.7287 \\
\hline & -10.2453 & 3.3519 & -4.4746 \\
\hline & $-8 . \varepsilon$ & & -5 \\
\hline & -0.6 & 2.4 & 5. \\
\hline & -1.6177 & 1.0 & 53 \\
\hline & 6.6479 & & 3.1908 \\
\hline & 5.2 & & \\
\hline & 0.1 & -2 & 6. \\
\hline & 0.8 & -1 & 36 \\
\hline & 2.1 & -3.7 & 6.0245 \\
\hline & 1.3 & & \\
\hline & 2.8 & -3.2 & 87 \\
\hline & 8.8 & -6 & -1.4132 \\
\hline & 9.4 & & \\
\hline & 4.6 & -6 & -2 \\
\hline & 0.1 & -6 . & -6 . \\
\hline & -0.9836 & -5.6 & -6.4901 \\
\hline & 3.8 & & \\
\hline & 10 & & 4 \\
\hline & -4.3 & 2 & \\
\hline C & 2.2 & 9 & -2.2948 \\
\hline C & & & \\
\hline ) & & & \\
\hline & -0.0112 & & -1 \\
\hline E & -1.3 & & -1.2592 \\
\hline & -1.5 & & \\
\hline ) & -1.7 & & \\
\hline & -2.7 & & -2 . \\
\hline C & -3.2 & 0 & -3.9345 \\
\hline & -2.7 & & \\
\hline : & -1.21 & & \\
\hline & & & 149 \\
\hline E & -0.7871 & 4.2175 & -5.7319 \\
\hline & 2.8 & & \\
\hline & 2.524 & & \\
\hline & & & 5889 \\
\hline & 3.34 & 2.9301 & $8<0$ \\
\hline & & & \\
\hline & $x_{3}+2$ & 1.0201 & 19.000 \\
\hline
\end{tabular}




\begin{tabular}{|c|c|c|c|c|c|c|c|c|c|c|c|}
\hline C & 2.6037 & 1.0781 & -1.7645 & C & -9.6997 & -3.0478 & -1.5070 & C & 5.1606 & -5.4180 & -3.796 \\
\hline 0 & 2.3226 & 2.0567 & -0.8074 & C & -8.7291 & 6.7436 & 3.8779 & 0 & 5.8413 & -6.4417 & -3.73 \\
\hline $\mathrm{H}$ & 2.4152 & 8.0406 & -1.8720 & C & -8.5965 & 5.4985 & 3.0139 & C & 4.3671 & -3.2647 & -5.026 \\
\hline $\mathrm{H}$ & 2.9916 & 6.3551 & -1.8912 & C & -8.5634 & 5.7682 & 1.5124 & $\mathrm{H}$ & 6.0619 & -4.5348 & -5.465 \\
\hline $\mathrm{H}$ & -1.4511 & 5.9836 & -0.5949 & $\mathrm{~N}$ & -8.0537 & 4.7242 & 0.7914 & $\mathrm{H}$ & 4.8830 & -2.6029 & -4.326 \\
\hline $\mathrm{H}$ & -1.9664 & 7.6623 & -0.8082 & 0 & -9.0034 & 6.7920 & 0.9986 & $\mathrm{H}$ & 3.3137 & -3.3330 & -4.732 \\
\hline $\mathrm{H}$ & -2.9976 & 2.8243 & -3.4283 & $\mathrm{H}$ & -10.2169 & -4.4820 & 3.0494 & H & 4.3898 & -2.7852 & -6.008 \\
\hline $\mathrm{H}$ & -3.2732 & 3.1153 & -5.1383 & $\mathrm{H}$ & -11.9508 & -4.6981 & 3.3604 & $\mathrm{~N}$ & 4.4425 & -4.9350 & -2.75 \\
\hline $\mathrm{H}$ & -0.7001 & 3.8122 & -3.6455 & $\mathrm{H}$ & -11.3716 & -3.2405 & 2.5390 & C & 4.3704 & -5.6535 & -1.51 \\
\hline $\mathrm{H}$ & 0.2779 & 4.0697 & -5.9332 & $\mathrm{H}$ & -12.3462 & -4.8719 & 0.8612 & C & 2.9325 & -6.0344 & -1.129 \\
\hline $\mathrm{H}$ & -1.3429 & 3.9166 & -6.6297 & $\mathrm{H}$ & -9.1209 & -2.2933 & -0.9642 & C & 2.1294 & -4.9203 & -0.43 \\
\hline $\mathrm{H}$ & -0.9498 & 5.2867 & -5.5678 & $\mathrm{H}$ & -7.7111 & 4.9168 & 3.2789 & C & 0.6359 & -5.2538 & -0.412 \\
\hline $\mathrm{H}$ & -1.2957 & 1.4487 & -5.4992 & $\mathrm{H}$ & -9.4543 & 4.8379 & 3.1909 & C & 2.6406 & -4.6774 & 0.990 \\
\hline $\mathrm{H}$ & -1.0675 & 1.3607 & -3.7457 & $\mathrm{H}$ & -7.4856 & 4.0202 & 1.2618 & $\mathrm{H}$ & 3.9363 & -4.0704 & -2.85 \\
\hline $\mathrm{H}$ & 0.2803 & 1.8401 & -4.7877 & $\mathrm{H}$ & -7.9129 & 4.8775 & -0.1962 & $\mathrm{H}$ & 4.8332 & -5.0636 & -0.711 \\
\hline $\mathrm{H}$ & -3.0186 & 5.6141 & -4.7150 & $\mathrm{H}$ & -9.5901 & 7.3409 & 3.5667 & $\mathrm{H}$ & 2.9627 & -6.9010 & -0.45 \\
\hline $\mathrm{H}$ & 2.0607 & 3.6316 & -2.5654 & $\mathrm{H}$ & -8.8488 & 6.4713 & 4.9311 & $\mathrm{H}$ & 2.4173 & -6.3663 & -2.04 \\
\hline $\mathrm{H}$ & 2.4265 & 4.6733 & -4.7400 & $\mathrm{H}$ & -7.8436 & 7. 3812 & 3.7913 & $\mathrm{H}$ & 2.2464 & -3.9904 & $-1.01]$ \\
\hline $\mathrm{H}$ & 3.5164 & 3.3932 & -6.5886 & $\mathrm{H}$ & -11.1992 & -6.1028 & 1.4101 & $\mathrm{H}$ & 0.4618 & -6.2028 & 0.11 \\
\hline $\mathrm{H}$ & 4.2485 & 1.0469 & -6.1752 & $\mathrm{H}$ & -10.2344 & -2.5624 & -2.3241 & $\mathrm{H}$ & 0.0648 & -4.4810 & 0.110 \\
\hline $\mathrm{H}$ & 3.8952 & 0.0113 & -3.9802 & $\mathrm{H}$ & -8.9986 & -3.7744 & -1.9258 & $\mathrm{H}$ & 0.2388 & -5.3593 & -1.42 \\
\hline C & -5.0268 & -3.4913 & 3.2342 & $\mathrm{H}$ & -11.6377 & -3.5183 & -0.7524 & $\mathrm{H}$ & 3.6551 & -4.2703 & 1.016 \\
\hline C & -3.5958 & -2.9425 & 3.3837 & C & 3.8828 & 0.2911 & -1.1291 & $\mathrm{H}$ & 2.6507 & -5.6172 & 1.55 \\
\hline 0 & -3.3475 & -1.7427 & 3.2693 & $\mathrm{H}$ & 3.9113 & -0.7108 & -1.5927 & $\mathrm{H}$ & 1.9945 & -3.9909 & 1.536 \\
\hline C & -5.9086 & -2.5659 & 2.3717 & 0 & 3.6490 & 0.2792 & 0.2164 & C & 9.2969 & -5.3811 & $-0.24 c$ \\
\hline C & -5.6620 & -2.7846 & 0.8956 & C & 5.1819 & 1.0426 & -1.4749 & C & 7.9438 & -5.2825 & -0.976 \\
\hline $\mathrm{N}$ & -6.5905 & -3.4452 & 0.1938 & $\mathrm{H}$ & 5.9995 & 0.5690 & -0.9315 & C & 7.7332 & -3.9540 & -1.669 \\
\hline 0 & -4.6076 & -2.3777 & 0.3480 & $\mathrm{H}$ & 5.4286 & 1.0312 & -2.5368 & C & 7.8260 & -3.8328 & $-3.06 e$ \\
\hline $\mathrm{N}$ & -2.6468 & -3.8396 & 3.7573 & $\mathrm{H}$ & 5.1032 & 2.0832 & -1.1440 & C & 7.4534 & -2.8032 & -0.922 \\
\hline C & -1.2893 & -3.3928 & 4.0602 & & & & & C & 7.6239 & -2.6003 & -3.685 \\
\hline C & -0.5180 & -3.0095 & 2.7906 & Int2 & $(\mathbf{A A})-\mathbf{S}$ & & & C & 7.2484 & -1.5681 & -1.538 \\
\hline 0 & -0.8772 & -3.4117 & 1.6756 & & & & & C & 7.3261 & -1.4649 & -2.92 \\
\hline C & -0.6441 & -4.6416 & 4.6931 & $C$ & 11.5020 & 5.2970 & -0.8992 & $\mathrm{H}$ & 9.3945 & -4.5964 & 0.50 \\
\hline C & -1.3185 & -5.7856 & 3.9240 & C & 9.9734 & 5.3221 & -0.7309 & $\mathrm{H}$ & 7.1420 & -5.4325 & -0.243 \\
\hline C & -2.7666 & -5.3037 & 3.7510 & C & 9.4356 & 4.2070 & 0.1189 & H & 7.8427 & -6.0886 & -1.70 \\
\hline $\mathrm{N}$ & 0.5856 & -2.2848 & 3.0314 & $\mathrm{C}$ & 8.4780 & 3.2673 & -0.1937 & $\mathrm{H}$ & 8.0281 & -4.7185 & -3.655 \\
\hline C & 1.6068 & -2.0867 & 2.0298 & $\mathrm{~N}$ & 9.8629 & 4.0211 & 1.4225 & $\mathrm{H}$ & 7. 3987 & -2.8816 & 0.158 \\
\hline C & 2.9765 & -2.5714 & 2.5277 & C & 9.1690 & 2.9925 & 1.8771 & $\mathrm{H}$ & 7.6964 & -2.5272 & -4.76 \\
\hline 0 & 3.1271 & -3.6446 & 3.1059 & $\mathrm{~N}$ & 8.3193 & 2.4929 & 0.9374 & $\mathrm{H}$ & 7.0386 & -0.6889 & -0.936 \\
\hline $\mathrm{N}$ & 3.9587 & -1.6840 & 2.2384 & $\mathrm{H}$ & 11.9841 & 5.3076 & 0.0819 & $\mathrm{H}$ & 7.1623 & -0.5077 & -3.415 \\
\hline C & 5.3715 & -1.9204 & 2.4755 & $\mathrm{H}$ & 9.4864 & 5.2870 & -1.7130 & C & -2.3823 & 1.0249 & 0.670 \\
\hline C & 5.9086 & -1.5256 & 3.8666 & $\mathrm{H}$ & 9.6872 & 6.2814 & -0.2787 & C & -1.2273 & 1.2231 & -0.042 \\
\hline 0 & 7.1202 & -1.6326 & 4.0732 & $\mathrm{H}$ & 7.9009 & 3.0946 & -1.0891 & C & -1.2053 & 2.3697 & -0.916 \\
\hline C & 6.2049 & -1.1684 & 1.4273 & $\mathrm{H}$ & 9.2314 & 2.5717 & 2.8708 & $\mathrm{~N}$ & -0.1116 & 2.7673 & -1.526 \\
\hline 0 & 5.9765 & 0.2378 & 1.4678 & $\mathrm{H}$ & 7.6294 & 1.7520 & 1.0802 & $\mathrm{~N}$ & -2.3494 & 3.0864 & -1.129 \\
\hline $\mathrm{N}$ & 5.0455 & -1.0599 & 4.7889 & C & -9.3602 & 1.8853 & -4.6059 & C & -3.4372 & 2.8088 & $-0.44 e$ \\
\hline C & 5.5457 & -0.6888 & 6.1064 & C & -8.5240 & 2.7095 & -3.6100 & $\mathrm{~N}$ & -3.4684 & 1.8329 & 0.490 \\
\hline $\mathrm{H}$ & -6.9640 & -2.7466 & 2.5966 & C & -7.4389 & 1.8904 & -2.9472 & $\mathrm{~S}$ & 1.3233 & -1.2772 & -3.263 \\
\hline $\mathrm{H}$ & -5.6633 & -1.5212 & 2.5957 & C & -6.2902 & 1.5193 & -3.6626 & C & 1.3795 & -0.2062 & -1.922 \\
\hline $\mathrm{H}$ & -5.0300 & -4.5107 & 2.8400 & C & -7.5569 & 1.4422 & -1.6263 & C & -4.6723 & 3.5983 & -0.710 \\
\hline $\mathrm{H}$ & -0.8890 & -4.6841 & 5.7592 & C & -5.3076 & 0.7192 & -3.0912 & $\mathrm{~N}$ & 0.3364 & -0.4310 & -1.10 \\
\hline $\mathrm{H}$ & 0.4418 & -4.6322 & 4.5883 & C & -6.5774 & 0.6444 & -1.0310 & C & -0.4983 & -1.5032 & -1.489 \\
\hline $\mathrm{H}$ & -1.2604 & -6.7485 & 4.4375 & c & -5.4489 & 0.2767 & -1.7703 & C & -1.6641 & -1.9246 & -0.669 \\
\hline $\mathrm{H}$ & -0.8518 & -5.8865 & 2.9407 & 0 & -4.4361 & -0.4682 & -1.2589 & C & -0.0947 & -2.0762 & -2.657 \\
\hline $\mathrm{H}$ & -3.4056 & -5.6460 & 4.5767 & $\mathrm{H}$ & -9.8486 & 1.0469 & -4.0984 & C & -0.7401 & -3.1914 & -3.418 \\
\hline $\mathrm{H}$ & -3.2020 & -5.6597 & 2.8126 & $\mathrm{H}$ & -9.1810 & 3.1359 & -2.8434 & C & 0.0036 & 0.3673 & 0.128 \\
\hline $\mathrm{H}$ & -1.3182 & -2.5381 & 4.7402 & $\mathrm{H}$ & -8.0754 & 3.5586 & -4.1411 & $\mathrm{H}$ & 0.8705 & 2.3850 & -1.305 \\
\hline $\mathrm{H}$ & 1.6851 & -1.0344 & 1.7526 & $\mathrm{H}$ & -6.1637 & 1.8627 & -4.6866 & H & -0.1418 & 3.6163 & -2.088 \\
\hline $\mathrm{H}$ & 1.3240 & -2.6628 & 1.1489 & H & -8.4161 & 1.7502 & -1.0357 & $\mathrm{H}$ & -0.1489 & -0.3509 & 0.93 \\
\hline $\mathrm{H}$ & 7.2536 & -1.3410 & 1.6567 & $\mathrm{H}$ & -4.4217 & 0.4303 & -3.6456 & H & 0.8788 & 0.9774 & $0.34 e$ \\
\hline $\mathrm{H}$ & 5.9931 & -1.5672 & 0.4267 & $\mathrm{H}$ & -6.6427 & 0.3777 & 0.0190 & $\mathrm{H}$ & -5.2557 & 3.7227 & 0.205 \\
\hline $\mathrm{H}$ & 5.0991 & 0.4056 & 1.0139 & $\mathrm{H}$ & -4.6561 & -0.8589 & -0.3911 & $\mathrm{H}$ & -4.3981 & 4.5614 & -1.142 \\
\hline $\mathrm{H}$ & 5.5742 & -2.9941 & 2.3773 & C & -0.1904 & -6.9864 & -4.4841 & $\mathrm{H}$ & -5.2914 & 3.0607 & -1.43 \\
\hline $\mathrm{H}$ & 6.3379 & 0.0581 & 6.0049 & $\mathrm{C}$ & -1.4115 & -7.5728 & -3.7511 & $\mathrm{H}$ & -2.5006 & 0.2345 & 1.407 \\
\hline $\mathrm{H}$ & 2.3902 & 7.0922 & -3.3782 & C & -2.3379 & -6.5209 & -3.1777 & $\mathrm{H}$ & -1.4119 & -2.0397 & 0.387 \\
\hline $\mathrm{H}$ & -4.3573 & 4.8463 & -3.8548 & C & -2.3575 & -6.2255 & -1.8099 & $\mathrm{H}$ & -1.9985 & -2.8978 & -1.02 \\
\hline $\mathrm{H}$ & -2.6335 & 4.7459 & -1.9138 & C & -3.1930 & -5.7846 & -4.0109 & $\mathrm{H}$ & -2.4908 & -1.2267 & -0.785 \\
\hline $\mathrm{H}$ & 0.1966 & 8.2712 & -1.2886 & C & -3.1737 & -5.2207 & -1.2858 & $\mathrm{H}$ & -0.0279 & -3.6816 & -4.086 \\
\hline $\mathrm{H}$ & 5.9669 & -1.5555 & 6.6292 & C & -4.0180 & -4.7840 & -3.5069 & $\mathrm{H}$ & -1.5750 & -2.8249 & -4.023 \\
\hline $\mathrm{H}$ & 4.7113 & -0.2783 & 6.6768 & C & -4.0073 & -4.4864 & -2.1381 & C & 1.8552 & -2.0954 & 7.675 \\
\hline $\mathrm{H}$ & 4.0392 & -1.1083 & 4.6508 & 0 & -4.8309 & -3.4962 & -1.6965 & C & 1.0498 & -0.8061 & 7.485 \\
\hline $\mathrm{H}$ & 0.8840 & -2.0265 & 3.9909 & $\mathrm{H}$ & 0.4279 & -6.3984 & -3.7978 & C & 1.8198 & 0.1880 & 6.608 \\
\hline $\mathrm{H}$ & -7.4477 & -3.8345 & 0.5793 & $\mathrm{H}$ & -1.9701 & -8.2095 & -4.4490 & 0 & 2.0156 & -0.2080 & 5.385 \\
\hline $\mathrm{H}$ & -6.4023 & -3.6083 & -0.7899 & $\mathrm{H}$ & -1.0652 & -8.2285 & -2.9445 & 0 & 2.2193 & 1.2567 & 7.082 \\
\hline $\mathrm{H}$ & 3.7262 & -0.9359 & 1.5759 & $\mathrm{H}$ & -1.7168 & -6.7846 & -1.1354 & C & -8.2637 & 3.1486 & 3.809 \\
\hline $\mathrm{H}$ & -5.4485 & -3.5465 & 4.2450 & $\mathrm{H}$ & -3.2076 & -5.9953 & -5.0777 & C & -7.3143 & 1.9918 & 3.479 \\
\hline C & -11.3499 & -5.0259 & 1.2905 & $\mathrm{H}$ & -3.1614 & -4.9988 & -0.2226 & C & -6.3426 & 2.2871 & 2.326 \\
\hline C & -10.2591 & -4.5045 & 0.3795 & H & -4.6700 & -4.2090 & -4.1561 & 0 & -6.3432 & 3.4425 & 1.837 \\
\hline 0 & -9.0710 & -4.7455 & 0.6126 & $\mathrm{H}$ & -4.5057 & -3.0916 & -0.8530 & 0 & -5.5847 & 1.3238 & 1.96 \\
\hline C & -11.2176 & -4.3192 & 2.6429 & C & 5.0438 & -4.6349 & -5.0780 & $\mathrm{H}$ & -8.9013 & 2.8946 & 4.663 \\
\hline & -10.6558 & -3.7163 & -0.6441 & & & & & & -7.7046 & 4.0530 & 4.05 \\
\hline
\end{tabular}




\begin{tabular}{|c|c|c|c|}
\hline $\mathrm{H}$ & -7.8710 & 1.0812 & 3.2230 \\
\hline $\mathrm{H}$ & -6.7017 & 1.7232 & 4.3489 \\
\hline C & -0.7777 & 2.4949 & 5.3257 \\
\hline C & -0.7079 & 1.8955 & 3.9094 \\
\hline $\mathrm{C}$ & 0.5580 & 2.2475 & 3.1946 \\
\hline $\mathrm{C}$ & 0.8248 & 3.0469 & 2.0997 \\
\hline $\mathrm{N}$ & 1.7954 & 1.8135 & 3.6255 \\
\hline 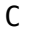 & 2.7288 & 2.3486 & 2.7956 \\
\hline $\mathrm{N}$ & 2.1824 & 3.1029 & 1.8534 \\
\hline $\mathrm{H}$ & 0.0752 & 2.1851 & 5.9354 \\
\hline $\mathrm{H}$ & -0.8219 & 0.8055 & 3.9647 \\
\hline $\mathrm{H}$ & -1.5529 & 2.2568 & 3.3126 \\
\hline 1 & 0.1180 & 3.5922 & 1.4891 \\
\hline $\mathrm{H}$ & 3.7848 & 2.1590 & 2.9162 \\
\hline $\mathrm{H}$ & 3.1308 & 1.4894 & 0.1798 \\
\hline C & 5.5183 & 7.4065 & 2.4482 \\
\hline C & 5.4970 & 6.1238 & 3.2859 \\
\hline C & 6.0753 & 4.8726 & 2.5966 \\
\hline C & 6.0782 & 3.6994 & 3.5888 \\
\hline C & 5.3114 & 4.5160 & 1.3139 \\
\hline $\mathrm{H}$ & 4.8860 & 7.3162 & 1.5595 \\
\hline $\mathrm{H}$ & 4.4624 & 5.9045 & 3.5842 \\
\hline $\mathrm{H}$ & 6.0527 & 6.2976 & 4.2174 \\
\hline $\mathrm{H}$ & 7. 1191 & 5.0825 & 2.3214 \\
\hline $\mathrm{H}$ & 5.0797 & 3.5468 & 4.0125 \\
\hline $\mathrm{H}$ & 6.3852 & 2.7629 & 3.1139 \\
\hline $\mathrm{H}$ & 6.7603 & 3.8900 & 4.4252 \\
\hline $\mathrm{H}$ & 4.2414 & 4.3722 & 1. 4971 \\
\hline $\mathrm{H}$ & 5.7018 & 3.5895 & 0.8823 \\
\hline $\mathrm{H}$ & 5.4240 & 5.2949 & 0.5521 \\
\hline $\mathrm{H}$ & -1.1363 & -3.9481 & -2.7408 \\
\hline $\mathrm{H}$ & -8.9075 & 3.3709 & 2.9579 \\
\hline $\mathrm{H}$ & 11.8224 & 4.3872 & -1.4178 \\
\hline $\mathrm{H}$ & 11.8521 & 6.1624 & -1.4724 \\
\hline $\mathrm{H}$ & -10.1341 & 2.4995 & -5.0782 \\
\hline $\mathrm{H}$ & -8.7261 & 1.4692 & -5.3952 \\
\hline $\mathrm{H}$ & -0.7670 & 3.5883 & 5.2764 \\
\hline $\mathrm{H}$ & -1.6982 & 2.1833 & 5.8308 \\
\hline $\mathrm{H}$ & 6.5349 & 7.6390 & 2.1092 \\
\hline $\mathrm{H}$ & 5.1548 & 8.2625 & 3.0273 \\
\hline $\mathrm{H}$ & 0.0937 & -1.0367 & 6.9963 \\
\hline $\mathrm{H}$ & 0.8310 & -0.3318 & 8.4460 \\
\hline $\mathrm{H}$ & 2.1318 & -2.5138 & 6.7033 \\
\hline $\mathrm{H}$ & 1.2923 & -2.8491 & 8.2374 \\
\hline $\mathrm{H}$ & 2.7820 & -1.8915 & 8.2219 \\
\hline $\mathrm{H}$ & 9.4020 & -6.3495 & 0.2496 \\
\hline $\mathrm{H}$ & 10.1274 & -5.2668 & -0.9540 \\
\hline $\mathrm{H}$ & 4.9823 & -6.5451 & -1.6525 \\
\hline $\mathrm{H}$ & 0.4338 & -7.7789 & -4.9087 \\
\hline $\mathrm{H}$ & -0.5047 & -6.3254 & -5.2986 \\
\hline $\mathrm{H}$ & 4.5240 & -5.2889 & -5.7896 \\
\hline $\mathrm{H}$ & 1.9718 & 1.1371 & 4.4015 \\
\hline $\mathrm{H}$ & -4.3761 & 1.6639 & 1.0924 \\
\hline C & 2.2581 & 6.7309 & -3.3579 \\
\hline C & 0.9457 & 6.1137 & -2.9516 \\
\hline 0 & 0.7259 & 4.9105 & -3.1200 \\
\hline $\mathrm{N}$ & 0.0228 & 6.9377 & -2.3983 \\
\hline C & -1.3381 & 6.4616 & -2.2255 \\
\hline C & -1.9196 & 6.0062 & -3.5764 \\
\hline 0 & -1.7158 & 6.6617 & -4.5957 \\
\hline $\mathrm{N}$ & -2.6598 & 4.8795 & -3.5024 \\
\hline C & -3.1903 & 4.2313 & -4.6896 \\
\hline C & -2.6400 & 2.8101 & -4.8554 \\
\hline C & -1.1156 & 2.7059 & -5.0096 \\
\hline C & -0.6895 & 1.2346 & -5.0270 \\
\hline C & -0.5982 & 3.4574 & -6.2408 \\
\hline C & 3.1216 & 1.0877 & -3.1502 \\
\hline C & 2.7244 & 2.2861 & -3.7512 \\
\hline C & 3.1711 & 2.6152 & -5.0301 \\
\hline C & 4.0249 & 1.7560 & -5.7253 \\
\hline C & 4.4431 & 0.5693 & -5.1239 \\
\hline C & 3.9969 & 0.2410 & -3.8425 \\
\hline C & 2.5794 & 0.7615 & -1.7417 \\
\hline 0 & 2.2321 & 1.8718 & -1.0508 \\
\hline $\mathrm{H}$ & 2.3859 & 7.7548 & -2.9988 \\
\hline $\mathrm{H}$ & 3.0703 & 6.1093 & -2.9749 \\
\hline $\mathrm{r}$ & -1.3631 & 5.6566 & -1.4866 \\
\hline $\mathrm{r}$ & -1.9412 & 7.2879 & -1.8413 \\
\hline F & -2.9504 & 2.2194 & -3.9844 \\
\hline & -3.1300 & 2.3540 & -5.7282 \\
\hline & & & \\
\hline
\end{tabular}

$\begin{array}{rrrrrrrr}\mathrm{H} & 0.4813 & 3.3114 & -6.3483 & \mathrm{H}-12.2648 & -4.6479 & 1.5669 \\ \mathrm{H} & -1.0784 & 3.0931 & -7.1587 & \mathrm{H} & -8.9882 & -2.4444 & -0.6143 \\ \mathrm{H} & -0.7822 & 4.5321 & -6.1571 & \mathrm{H} & -7.7393 & 5.4404 & 2.3426 \\ \mathrm{H} & -1.1145 & 0.7079 & -5.8914 & \mathrm{H} & -9.4766 & 5.2848 & 2.2529 \\ \mathrm{H} & -1.0295 & 0.7173 & -4.1223 & \mathrm{H} & -7.4900 & 4.1799 & 0.5147 \\ \mathrm{H} & 0.3990 & 1.1430 & -5.0769 & \mathrm{H} & -7.9066 & 4.7676 & -1.0745 \\ \mathrm{H} & -2.9412 & 4.8684 & -5.5396 & \mathrm{H} & -9.7045 & 7.8072 & 2.1751 \\ \mathrm{H} & 2.0905 & 2.9753 & -3.2095 & \mathrm{H} & -8.9552 & 7.2226 & 3.6802 \\ \mathrm{H} & 2.8509 & 3.5523 & -5.4756 & \mathrm{H} & -7.9644 & 7.9520 & 2.4054 \\ \mathrm{H} & 4.3722 & 2.0155 & -6.7211 & \mathrm{H}-11.1498 & -5.8001 & 2.3163 \\ \mathrm{H} & 5.1233 & -0.1006 & -5.6418 & \mathrm{H}-10.0731 & -2.9428 & -1.9318 \\ \mathrm{H} & 4.3527 & -0.6743 & -3.3839 & \mathrm{H} & -8.8521 & -4.0692 & -1.3029 \\ \mathrm{C} & -4.9702 & -2.8196 & 3.7896 & \mathrm{H}-11.5134 & -3.6004 & -0.2427 \\ \mathrm{C} & -3.5272 & -2.2765 & 3.7950 & \mathrm{C} & 3.7319 & 0.0216 & -0.8702 \\ \mathrm{O} & -3.2856 & -1.0986 & 3.5345 & \mathrm{O} & 3.6827 & 0.6538 & 0.3933 \\ \mathrm{C} & -5.8502 & -2.1136 & 2.7375 & \mathrm{C} & 3.6259 & -1.4888 & -0.7218 \\ \mathrm{C} & -5.5787 & -2.6271 & 1.3393 & \mathrm{H} & 2.6584 & -1.7847 & -0.3107 \\ \mathrm{~N} & -6.4838 & -3.4368 & 0.7767 & \mathrm{H} & 3.7631 & -1.9889 & -1.6836 \\ \mathrm{O} & -4.5266 & -2.3169 & 0.7284 & \mathrm{H} & 4.3971 & -1.8616 & -0.0486 \\ \mathrm{~N} & -2.5530 & -3.1276 & 4.2163 & \mathrm{H} & 4.6834 & 0.2678 & -1.3572\end{array}$

Int2(AA)-R

\begin{tabular}{|c|c|c|c|}
\hline & 11.4631 & 155 & -0.2 \\
\hline & 9.9410 & & -0 . \\
\hline & 9.4309 & 965 & 0.600 \\
\hline & 2057 & 2021 & a 10 \\
\hline & & & \\
\hline & 349 & 82 & \\
\hline & 8.2725 & 915 & 11 \\
\hline & 11.9814 & & \\
\hline & & & \\
\hline & 32 & & \\
\hline & 7.7120 & 311 & -0.658 \\
\hline & & & \\
\hline & & & \\
\hline & -9.4 & & -4 \\
\hline & -8.6 & & \\
\hline & -7 & & -2 \\
\hline & -6.2 & & -3 \\
\hline & -7.6677 & 1. & -1.298 \\
\hline & -5.2 & & -2 . \\
\hline & -6 & & -0 . \\
\hline & -5.5 & & -1 \\
\hline & -4.4817 & -0. & -1.25 \\
\hline & -9. & & \\
\hline & -9 & & -2 \\
\hline & -8.2 & & -3 \\
\hline & -6.2 & & -4 \\
\hline & & & \\
\hline & -4 & & -3 \\
\hline & -6.7 & & \\
\hline & -4 & -0. & -0. \\
\hline & -0. & & \\
\hline & -1. & -7 & -4 . \\
\hline & -2.1 & -6 . & -3 \\
\hline & -2 & & -2 . \\
\hline & & & \\
\hline & -2.8 & -4 & -1 \\
\hline & -3.9 & -4 & -3 \\
\hline & -3. & & -2 \\
\hline & & & \\
\hline & 0 & & -5 \\
\hline & -1.5 & -7 & -5 . \\
\hline & -0. & & -3 \\
\hline & & & \\
\hline & -3.2 & & \\
\hline & -2 & & \\
\hline & -4 & & \\
\hline & -4 . & & \\
\hline r & 4.8 & & -5 \\
\hline C & & & -4 \\
\hline 0 & & & \\
\hline & & & \\
\hline & 5.6 & & -6 \\
\hline & & & -5 \\
\hline & & & . 8122 \\
\hline & & & \\
\hline & & & -3.8392 \\
\hline & 42 & 61 & \\
\hline
\end{tabular}




\begin{tabular}{|c|c|c|c|c|c|c|c|c|c|c|c|}
\hline C & 2.9227 & -5.5461 & -1.7449 & $\mathrm{H}$ & 0.0335 & 0.9564 & 6.1701 & $\mathrm{H}$ & 3.7527 & 3.1727 & -6.7881 \\
\hline C & 2.2804 & -4.1690 & -1.4933 & $\mathrm{H}$ & -0.8076 & 0.0756 & 3.9096 & $\mathrm{H}$ & 4.6535 & 0.9782 & -6.0282 \\
\hline C & 1.0753 & -4.3374 & -0.5642 & $\mathrm{H}$ & -1.4332 & 1.6717 & 3.5493 & $\mathrm{H}$ & 4.1498 & 0.1500 & -3.773 \\
\hline C & 3.2737 & -3.1491 & -0.9233 & $\mathrm{H}$ & 0.3668 & 3.0405 & 1.8943 & $\mathrm{C}$ & -5.0320 & -3.5392 & 3.267 \\
\hline $\mathrm{H}$ & 3.2250 & -4.4006 & -4.0307 & $\mathrm{H}$ & 3.9049 & 1.3248 & 3.3417 & C & -3.6096 & -3.0014 & 3.49 \\
\hline $\mathrm{H}$ & 4.9997 & -5.0050 & -1.9749 & $\mathrm{H}$ & 3.0959 & 1.4491 & 0.5826 & 0 & -3.3934 & -1.7985 & 3.62 \\
\hline $\mathrm{H}$ & 3.1339 & -6.0117 & -0.7746 & C & 5.5341 & 6.6923 & 3.4907 & C & -5.8894 & -2.5590 & 2.444 \\
\hline $\mathrm{H}$ & 2.1899 & -6.1924 & -2.2475 & C & 5.6057 & 5.3216 & 4.1701 & C & -5.5776 & -2.6718 & 0.971 \\
\hline $\mathrm{H}$ & 1.9087 & -3.7787 & -2.4540 & C & 6.1943 & 4.1883 & 3.3085 & $\mathrm{~N}$ & -6.5261 & -3.1670 & 0.166 \\
\hline $\mathrm{H}$ & 1.4045 & -4.5955 & 0.4478 & C & 6.2356 & 2.8904 & 4.1285 & 0 & -4.4582 & -2.3184 & 0.522 \\
\hline $\mathrm{H}$ & 0.4866 & -3.4240 & -0.4839 & C & 5.4117 & 3.9947 & 2.0030 & $\mathrm{~N}$ & -2.6244 & -3.9259 & 3.659 \\
\hline $\mathrm{H}$ & 0.4042 & -5.1264 & -0.9199 & $\mathrm{H}$ & 4.8534 & 6.6806 & 2.6342 & C & -1.2723 & -3.4932 & 3.995 \\
\hline $\mathrm{H}$ & 4.0892 & -2.9274 & -1.6182 & $\mathrm{H}$ & 4.5960 & 5.0238 & 4.4854 & $C$ & -0.5598 & -2.8774 & 2.786 \\
\hline $\mathrm{H}$ & 3.7104 & -3.5216 & 0.0082 & $\mathrm{H}$ & 6.1997 & 5.4065 & 5.0901 & 0 & -0.9353 & -3.1119 & 1.632 \\
\hline $\mathrm{H}$ & 2.7736 & -2.2039 & -0.6924 & $\mathrm{H}$ & 7.2294 & 4.4532 & 3.0465 & C & -0.5704 & -4.8119 & 4.376 \\
\hline C & 9.1682 & -5.5086 & -1.3059 & H & 5.2402 & 2.6453 & 4.5151 & C & -1.2235 & -5.8183 & 3.416 \\
\hline C & 7.8097 & -4.7981 & -1.3546 & $\mathrm{H}$ & 6.5780 & 2.0415 & 3.5305 & C & -2.6887 & -5.3572 & 3.335 \\
\hline C & 7.8738 & -3.2832 & -1.4121 & $\mathrm{H}$ & 6.9049 & 2.9891 & 4.9907 & $\mathrm{~N}$ & 0.5295 & -2.1550 & 3.106 \\
\hline C & 7.0238 & -2.5802 & -2.2779 & $\mathrm{H}$ & 4.3453 & 3.8297 & 2.1851 & C & 1.5159 & -1.7821 & 2.126 \\
\hline C & 8.7443 & -2.5419 & -0.6023 & $\mathrm{H}$ & 5.7902 & 3.1261 & 1.4602 & C & 2.8616 & -2.4464 & $2.41 \varepsilon$ \\
\hline C & 7.0369 & -1.1854 & -2.3299 & $\mathrm{H}$ & 5.5162 & 4.8592 & 1.3386 & 0 & 2.9710 & -3.6557 & 2.612 \\
\hline C & 8.7690 & -1.1477 & -0.6574 & $\mathrm{H}$ & -1.5500 & -2.5542 & -3.9908 & $\mathrm{~N}$ & 3.8876 & -1.5642 & 2.41 \\
\hline C & 7.9144 & -0.4625 & -1.5209 & $\mathrm{H}$ & -8.9148 & 2.7244 & 3.4964 & $\mathrm{C}$ & 5.2861 & -1.8961 & 2.58 \\
\hline $\mathrm{H}$ & 9.7383 & -5.2507 & -0.4079 & $\mathrm{H}$ & 11.7762 & 4.3506 & -0.9459 & $\mathrm{C}$ & 5.8763 & -1.4527 & 3.936 \\
\hline $\mathrm{H}$ & 7.2239 & -5.0934 & -0.4718 & $\mathrm{H}$ & 11.7785 & 6.0937 & -0.6056 & 0 & 7.0981 & -1.4610 & 4.093 \\
\hline $\mathrm{H}$ & 7.2583 & -5.1552 & -2.2290 & $\mathrm{H}$ & -10.2618 & 3.1674 & -4.5576 & $\mathrm{C}$ & 6.1221 & -1.2646 & 1.463 \\
\hline $\mathrm{H}$ & 6.3624 & -3.1420 & -2.9275 & $\mathrm{H}$ & -8.8064 & 2.2737 & -5.0340 & 0 & 6.0062 & 0.1604 & 1.479 \\
\hline $\mathrm{H}$ & 9.4081 & -3.0558 & 0.0866 & $\mathrm{H}$ & -0.7357 & 2.4981 & 5.7990 & $\mathrm{~N}$ & 5.0047 & -1.0570 & 4.885 \\
\hline $\mathrm{H}$ & 6.3713 & -0.6621 & -3.0114 & $\mathrm{H}$ & -1.7308 & 1.0393 & 5.9741 & $\mathrm{C}$ & 5.5005 & -0.6356 & 6.185 \\
\hline $\mathrm{H}$ & 9.4424 & -0.5896 & -0.0141 & $\mathrm{H}$ & 6.5203 & 7.0039 & 3.1257 & $\mathrm{H}$ & -6.9515 & -2.7570 & 2.613 \\
\hline $\mathrm{H}$ & 7.9378 & 0.6208 & -1.5585 & $\mathrm{H}$ & 5.1778 & 7.4599 & 4.1863 & $\mathrm{H}$ & -5.6587 & -1.5306 & 2.746 \\
\hline C & -2.3615 & 0.9974 & 0.7791 & $\mathrm{H}$ & 0.0819 & -2.2882 & 6.8285 & $\mathrm{H}$ & -5.0313 & -4.5331 & 2.813 \\
\hline C & -1.2521 & 1.3407 & 0.0511 & $\mathrm{H}$ & 0.9639 & -1.8932 & 8.3128 & $\mathrm{H}$ & -0.7914 & -5.0584 & 5.419 \\
\hline C & -1.3170 & 2.5940 & -0.6588 & $\mathrm{H}$ & 2.0084 & -3.7459 & 6.0837 & $\mathrm{H}$ & 0.5126 & -4.7456 & 4.254 \\
\hline $\mathrm{N}$ & -0.2862 & 3.0851 & -1.2993 & H & 1.2953 & -4.3392 & 7.6023 & $\mathrm{H}$ & -1.1341 & -6.8550 & 3.751 \\
\hline $\mathrm{N}$ & -2.4854 & 3.3075 & -0.6746 & $\mathrm{H}$ & 2.8214 & -3.4425 & 7.6198 & $\mathrm{H}$ & -0.7603 & -5.7315 & 2.430 \\
\hline C & -3.5239 & 2.8941 & 0.0173 & $\mathrm{H}$ & 9.0301 & -6.5943 & -1.3081 & $\mathrm{H}$ & -3.3188 & -5.8913 & 4.059 \\
\hline $\mathrm{N}$ & -3.4824 & 1.7764 & 0.7845 & $\mathrm{H}$ & 9.7762 & -5.2424 & -2.1763 & $\mathrm{H}$ & -3.1048 & -5.5163 & 2.336 \\
\hline $\mathrm{S}$ & 1.5097 & -0.7915 & -3.3902 & $\mathrm{H}$ & 4.5945 & -6.5579 & -2.6748 & $\mathrm{H}$ & -1.3005 & -2.7693 & 4.813 \\
\hline C & 1.4716 & 0.2230 & -1.9981 & $\mathrm{H}$ & 0.2306 & -7.0676 & -6.1671 & $\mathrm{H}$ & 1.6273 & -0.6981 & 2.076 \\
\hline C & -4.7838 & 3.6864 & -0.0535 & $\mathrm{H}$ & -0.9640 & -5.7754 & -6.3710 & $\mathrm{H}$ & 1.1734 & -2.1429 & $1.15 e$ \\
\hline $\mathrm{N}$ & 0.3913 & -0.0793 & -1.2561 & $\mathrm{H}$ & 3.8990 & -4.2065 & -6.3373 & $\mathrm{H}$ & 7.1679 & -1.5009 & 1.646 \\
\hline C & -0.4096 & -1.1263 & -1.7538 & $\mathrm{H}$ & 1.9940 & 0.2323 & 4.6147 & $\mathrm{H}$ & 5.8277 & -1.6680 & $0.48 \varepsilon$ \\
\hline C & -1.6363 & -1.5966 & -1.0601 & $\mathrm{H}$ & -4.3612 & 1.4758 & 1.3657 & $\mathrm{H}$ & 5.1416 & 0.4049 & 1.085 \\
\hline C & 0.0714 & -1.6335 & -2.9208 & C & 2.2070 & 6.9993 & -2.3086 & $\mathrm{H}$ & 5.3670 & -2.9867 & 2.526 \\
\hline C & -0.5001 & -2.7410 & -3.7513 & C & 0.7966 & 6.5045 & -2.1545 & $\mathrm{H}$ & 6.2765 & 0.1230 & 6.055 \\
\hline C & 0.0293 & 0.5450 & 0.0656 & 0 & 0.4629 & 5.4069 & -2.6096 & $\mathrm{H}$ & 2.4620 & 7.0003 & -3.371 \\
\hline $\mathrm{H}$ & 0.7634 & 2.7166 & -1.1951 & $\mathrm{~N}$ & -0.0768 & 7.2857 & -1.4817 & $\mathrm{H}$ & -4.3707 & 4.7227 & -3.872 \\
\hline $\mathrm{H}$ & -0.3924 & 3.9926 & -1.7465 & C & -1.4685 & 6.8843 & -1.3646 & $\mathrm{H}$ & -2.6718 & 4.7378 & -1.940 \\
\hline $\mathrm{H}$ & -0.0567 & -0.2759 & 0.7725 & C & -2.0497 & 6.5240 & -2.7453 & $\mathrm{H}$ & 0.1891 & 8.2265 & -1.240 \\
\hline $\mathrm{H}$ & 0.8744 & 1.1713 & 0.3378 & 0 & -1.8998 & 7.2730 & -3.7063 & $\mathrm{H}$ & 5.9412 & -1.4740 & 6.738 \\
\hline $\mathrm{H}$ & -5.2786 & 3.7037 & 0.9211 & $\mathrm{~N}$ & -2.7317 & 5.3595 & -2.7517 & $\mathrm{H}$ & 4.6614 & -0.2227 & 6.747 \\
\hline $\mathrm{H}$ & -4.5564 & 4.6911 & -0.4103 & C & -3.2778 & 4.7905 & -3.9660 & $\mathrm{H}$ & 3.9995 & -1.1924 & 4.783 \\
\hline $\mathrm{H}$ & -5.4692 & 3.2126 & -0.7623 & C & -2.7322 & 3.3893 & -4.2669 & $\mathrm{H}$ & 0.8361 & -2.0082 & 4.080 \\
\hline $\mathrm{H}$ & -2.4195 & 0.0989 & 1.3870 & C & -1.2439 & 3.3044 & -4.6369 & $\mathrm{H}$ & -7.3927 & -3.5815 & 0.505 \\
\hline H & -1.4664 & -1.8221 & -0.0046 & C & -0.8192 & 1.8358 & -4.7482 & $\mathrm{H}$ & -6.2814 & -3.3027 & -0.809 \\
\hline $\mathrm{H}$ & -1.9450 & -2.5273 & -1.5296 & C & -0.9203 & 4.0827 & -5.9166 & $\mathrm{H}$ & 3.6655 & -0.6050 & 2.189 \\
\hline H & -2.4496 & -0.8796 & -1.1662 & C & 2.9142 & 1.8421 & -3.2228 & $\mathrm{H}$ & -5.4786 & -3.6490 & 4.262 \\
\hline $\mathrm{H}$ & -0.4577 & -3.6948 & -3.2195 & C & 2.4443 & 3.0855 & -3.6425 & C & -11.1331 & -5.2230 & 1.342 \\
\hline $\mathrm{H}$ & 0.0445 & -2.8499 & -4.6924 & C & 2.7420 & 3.5608 & -4.9205 & C & -10.0948 & -4.5738 & 0.453 \\
\hline C & 1.8402 & -3.5096 & 7.1376 & C & 3.5218 & 2.8022 & -5.7936 & 0 & -8.9030 & -4.5713 & 0.774 \\
\hline C & 1.0842 & -2.1869 & 7.2669 & C & 4.0208 & 1.5683 & -5.3713 & $\mathrm{C}$ & -11.2126 & -4.4164 & 2.641 \\
\hline C & 1.8227 & -1.0729 & 6.5225 & C & 3.7239 & 1.0964 & -4.0933 & $\mathrm{~N}$ & -10.5544 & -3.9425 & -0.649 \\
\hline 0 & 2.0075 & -1.2978 & 5.2547 & C & 2.5432 & 1.3580 & -1.7948 & C & -9.6751 & -3.1722 & -1.509 \\
\hline 0 & 2.2037 & -0.0618 & 7.1231 & 0 & 2.0765 & 2.3382 & -0.9961 & C & -8.8104 & 6.6746 & 3.791 \\
\hline C & -8.2712 & 2.3695 & 4.3018 & $\mathrm{H}$ & 2.3733 & 7.9977 & -1.8963 & $\mathrm{C}$ & -8.6630 & 5.4036 & 2.970 \\
\hline C & -7.2966 & 1.3057 & 3.7829 & $\mathrm{H}$ & 2.8747 & 6.2905 & -1.8109 & C & -8.6598 & 5.6195 & 1.460 \\
\hline C & -6.3138 & 1.8243 & 2.7228 & $\mathrm{H}$ & -1.5639 & 6.0473 & -0.6650 & $\mathrm{~N}$ & -8.1159 & 4.5717 & 0.771 \\
\hline 0 & -6.3080 & 3.0567 & 2.4831 & $\mathrm{H}$ & -2.0311 & 7.7295 & -0.9616 & 0 & -9.1517 & 6.6036 & 0.915 \\
\hline 0 & -5.5568 & 0.9538 & 2.1723 & $\mathrm{H}$ & -2.9229 & 2.7530 & -3.3949 & $\mathrm{H}$ & -10.2267 & -4.3691 & 3.108 \\
\hline $\mathrm{H}$ & -8.9094 & 1.9573 & 5.0920 & $\mathrm{H}$ & -3.3299 & 2.9681 & -5.0891 & $\mathrm{H}$ & -11.9120 & -4.8727 & 3.347 \\
\hline $\mathrm{H}$ & -7.7309 & 3.2266 & 4.7043 & $\mathrm{H}$ & -0.6639 & 3.7586 & -3.8317 & $\mathrm{H}$ & -11.5466 & -3.3923 & 2.447 \\
\hline $\mathrm{H}$ & -7.8327 & 0.4488 & 3.3559 & H & 0.1274 & 3.9370 & -6.1972 & $\mathrm{H}$ & -12.1112 & -5.2783 & 0.851 \\
\hline $\mathrm{H}$ & -6.6916 & 0.8928 & 4.6001 & $\mathrm{H}$ & -1.5417 & 3.7444 & -6.7564 & $\mathrm{H}$ & -9.1743 & -2.3742 & -0.953 \\
\hline C & -0.7755 & 1.4221 & 5.6014 & $\mathrm{H}$ & -1.0808 & 5.1559 & -5.7785 & $\mathrm{H}$ & -7.7612 & 4.8512 & 3.242 \\
\hline C & -0.6425 & 1.1448 & 4.0945 & $\mathrm{H}$ & -1.3720 & 1.3222 & -5.5457 & $\mathrm{H}$ & -9.5044 & 4.7330 & 3.184 \\
\hline C & 0.6755 & 1.5561 & 3.5146 & H & -1.0132 & 1.3014 & -3.8113 & $\mathrm{H}$ & -7.5124 & 3.9099 & 1.259 \\
\hline C & 1.0197 & 2.4210 & 2.4933 & $\mathrm{H}$ & 0.2489 & 1.7511 & -4.9661 & $\mathrm{H}$ & -7.9963 & 4.6899 & -0.223 \\
\hline $\mathrm{N}$ & 1.8746 & 1.0345 & 3.9548 & $\mathrm{H}$ & -3.0640 & 5.4918 & -4.7747 & $\mathrm{H}$ & -9.6866 & 7.2438 & 3.469 \\
\hline C & 2.8625 & 1.5701 & 3.1968 & $\mathrm{H}$ & 1.8599 & 3.6914 & -2.9644 & $\mathrm{H}$ & -8.9128 & 6.4376 & 4.855 \\
\hline $\mathrm{N}$ & 2.3861 & 2.4145 & 2.2893 & $\mathrm{H}$ & 2.3562 & 4.5289 & -5.2254 & $\mathrm{H}$ & -7.9387 & 7. 3262 & 3.672 \\
\hline
\end{tabular}




$\begin{array}{rrrr}\text { H } & -10.8039 & -6.2452 & 1.5506 \\ \mathrm{H} & -10.2632 & -2.7269 & -2.3125 \\ \mathrm{H} & -8.9052 & -3.8127 & -1.9505 \\ \mathrm{H} & -11.5426 & -3.9596 & -0.8438 \\ \mathrm{C} & 3.8336 & 0.7794 & -1.0915 \\ \mathrm{H} & 4.1101 & -0.2080 & -1.4739 \\ \mathrm{O} & 3.5165 & 0.6013 & 0.2881 \\ \mathrm{C} & 4.9979 & 1.7629 & -1.2243 \\ \mathrm{H} & 5.8229 & 1.4175 & -0.6026 \\ \mathrm{H} & 5.3542 & 1.8506 & -2.2511 \\ \mathrm{H} & 4.6704 & 2.7469 & -0.8798\end{array}$

\section{TS3(AA)-S}

\begin{tabular}{|c|c|c|c|}
\hline & 058 & 23 & -0.9475 \\
\hline & 10.0880 & 4.9965 & -0.7135 \\
\hline & & 07 & 6 \\
\hline & 81 & 702 & -0 \\
\hline & 10.1024 & 4714 & 1.2873 \\
\hline 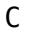 & 3903 & 4358 & 598 \\
\hline & 288 & & 3018 \\
\hline & 12.1257 & 626 & 098 \\
\hline $\mathrm{H}$ & 9.5584 & 570 & -1.6720 \\
\hline 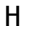 & 9. & & \\
\hline H & 12 & & -1 \\
\hline $\mathrm{H}$ & 184 & 074 & 2.6331 \\
\hline $\mathrm{H}$ & & & \\
\hline C & & & \\
\hline C & -8. & & -3 \\
\hline & -7 & & -2 \\
\hline & & & \\
\hline & -7 & & \\
\hline & -5.4142 & & -2 \\
\hline & -6. & & 077 \\
\hline & & & \\
\hline 0 & -4 & -0 . & \\
\hline & -9. & & \\
\hline & -9. & & \\
\hline & -8 & & \\
\hline & -6 & 2. & -4 \\
\hline & -8.2 & 2. & -0 . \\
\hline & -4 & & \\
\hline & -6 & & \\
\hline & -4 & -0. & -0. \\
\hline( & -0 . & -6 & -4 \\
\hline & & & \\
\hline & -2 & -6 . & \\
\hline & -2 & -6 . & -2 \\
\hline $\mathrm{C}$ & -3 & -5 . & -4 \\
\hline & & & \\
\hline & -4 & -4 . & \\
\hline & -4 & -4 & -2 \\
\hline 0 & -5 & $7+7>$ & -1 \\
\hline & & & \\
\hline & -2 & -7 . & \\
\hline & -1.6 & -8 & -3 \\
\hline & -2 & & \\
\hline & -3 & & \\
\hline & -3.2 & -5 . & -0 \\
\hline & -4. & -3 & 466 \\
\hline & -4 & & \\
\hline & & & \\
\hline C & & -4 . & -4 \\
\hline 0 & 4. & -3 & -3 \\
\hline C & & & \\
\hline & & & \\
\hline & & -2 . & \\
\hline F & & -2 & \\
\hline & & & \\
\hline & & & \\
\hline & & -5 . & \\
\hline C & & & \\
\hline 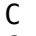 & & & \\
\hline & & & \\
\hline & & -4 & \\
\hline & & & \\
\hline & & & \\
\hline & & & \\
\hline & & & \\
\hline & & & \\
\hline
\end{tabular}

$\mathrm{H}$
$\mathrm{H}$
$\mathrm{H}$
$\mathrm{H}$
$\mathrm{C}$
$\mathrm{C}$
$\mathrm{C}$
$\mathrm{C}$

$$
\begin{array}{r}
0.79 \\
-0.42 \\
0.28 \\
2.48 \\
2.29 \\
0.8 \\
9.07 \\
8.05 \\
8.09 \\
7.18 \\
9.05
\end{array}
$$

0.2835

2.4835

2.2987

0.8897
9.0799

8.0511

8.0968

9.1841

7.2147

9.0858

8.1619

10.0976

8.2215

7.0446

6.4253

9.7598

6.4869

9.8075

8.1811

$-2.3863$

$-1.2454$

$-1.1971$

$-0.1105$

$-2.3072$

$-3.3884$

$-3.4371$

1.1603

1. 2269

$-4.5940$

0.1741

$-0.6858$

$-1.8573$

$-0.2799$

$-0.9053$

$-0.0658$

0.8462

$-0.1230$

$-0.2235$

0.8525

$-5.1323$

$-4.2936$

$-5.2706$

$-2.5174$

$-1.5970$

$-2.2369$

$-2.6583$

$-0.1547$

$-1.6679$

1.9957

1.2134

1. 9866

2. 1716

2. 3982

$-8.0640$

$-7.1696$

$-6.2340$

$-6.2160$

$-5.5232$

$-8.6762$

$-7.4639$

$-7.7685$

$-6.5328$

$-0.5597$

$-0.5231$

0.7539

1.0469

1.9762

2. 9268

2.4075

0.2812

$-0.6880$

$-1.3538$

0.3621

3.9743

3. 3541

5.7990

5.8134

6.3361
0.3520

$-0.6265$

$-7.2564-1.1871$

$-3.5306-1.2710$

$\begin{array}{ll}-4.3276 & 0.3059\end{array}$

$-3.6627-0.5211$

$-5.6741-0.9729$

$-4.7727-0.2700$

$-3.3333-0.7364$

$-2.8574-1.6849$

$-2.4450-0.2268$

$-1.5257-2.1042$

$-1.1130-0.6378$

$-0.6479-1.5760$

$-5.3098-0.8019$

$\begin{array}{ll}4.8090 & 0.8125\end{array}$

$-5.1731-0.4383$

$-3.5180-2.0894$

$-1.1764-2.8295$

$-0.4265-0.2066$

$0.3942-1.8789$

$0.9590 \quad 0.7313$

$1.1616-0.0034$

$2.3717-0.7780$

$2.7836-1.4040$

$3.1570-0.8916$

$2.8722-0.1920$

$\begin{array}{ll}1.8259 & 0.6654\end{array}$

$-1.1978-3.4200$

$-0.1933-2.0326$

$3.7332-0.3582$

$-0.4880-1.2546$

$-1.5254-1.6916$

$-1.9635-0.8877$

$-2.0301-2.8868$

$-3.1192-3.6990$

$0.2211 \quad 0.0354$

$2.4088-1.2606$

$3.6806-1.8879$

$-0.5323 \quad 0.8052$

$0.7618 \quad 0.2658$

$\begin{array}{ll}0.8263 & 0.5893\end{array}$

$\begin{array}{ll}4.7074 & -0.7444\end{array}$

$3.2682-1.0819$

$0.1155 \quad 1.4048$

$\begin{array}{ll}-2.1572 & 0.1550\end{array}$

$-2.8956-1.3036$

$-1.2281-0.9401$

$-3.6394 \quad-4.2995$

$-2.7283-4.3806$

$-2.7374 \quad 7.4039$

$-1.4253 \quad 7.3263$

$-0.4015 \quad 6.4914$

$-0.7432 \quad 5.2513$

$0.6435 \quad 7.0085$

$3.0672 \quad 4.2499$

$1.9093 \quad 3.7928$

$2.2572 \quad 2.6245$

$3.4437 \quad 2.2161$

$1.2991 \quad 2.1644$

$2.7683 \quad 5.1086$

$3.9315 \quad 4.5373$

$1.0395 \quad 3.4936$

$1.5550 \quad 4.6130$

$2.0844 \quad 5.4689$

$1.5838 \quad 4.0113$

$1.9166 \quad 3.3044$

$2.7059 \quad 2.2085$

$1.4428 \quad 3.7346$

$1.9419 \quad 2.9092$

$2.7134 \quad 1.9637$

$1.6950 \quad 6.0485$

$0.4986 \quad 3.9917$

$2.0221 \quad 3.4470$

$3.2770 \quad 1.5969$

$\begin{array}{ll}1.7067 & 3.0261\end{array}$

$\begin{array}{ll}1.6023 & 0.3703\end{array}$

$\begin{array}{ll}6.9972 & 2.7350\end{array}$

$\begin{array}{ll}5.6778 & 3.5127\end{array}$

\begin{tabular}{|c|c|c|c|}
\hline & 6.3583 & 3.2315 & 3.6626 \\
\hline & 5.5089 & 4.1798 & 1.4709 \\
\hline & 5.1116 & 6.9549 & 1.8845 \\
\hline & 4.7956 & 5.4539 & 3.8614 \\
\hline & 6.4246 & 5.8024 & 4.4169 \\
\hline & 7.3712 & 4.6602 & 2.4237 \\
\hline & 5.3672 & 3.0564 & 4.0955 \\
\hline & 6.6539 & 2.3227 & 132 \\
\hline & 7.0553 & 3.3807 & 4.4952 \\
\hline & 4.4445 & 4.0584 & 1.6960 \\
\hline & 5.8491 & 3.2619 & 0.983 \\
\hline & 5.6117 & 4.9881 & \\
\hline & -1.3882 & -3.8597 & -3.3690 \\
\hline & -8.7322 & 3.3759 & \\
\hline & 11.8814 & 4.0856 & -1.557 \\
\hline & 11.9564 & 5.8571 & -1.455 \\
\hline & -10.2210 & 3.1023 & -4.6122 \\
\hline & -8.8704 & 2.0266 & -5 \\
\hline & -0.5016 & 3.1772 & 5. \\
\hline & -1.4917 & 1.7774 & 5. \\
\hline & 6.7955 & 7.2353 & \\
\hline & 5.4835 & 7.8293 & 3. \\
\hline & 0.2395 & -1.6018 & 6.8 \\
\hline & 1.0336 & -1.0086 & 8. \\
\hline & 2.2304 & -3.0958 & \\
\hline & 1.4375 & -3.5171 & 7. \\
\hline & 2.9448 & -2.5876 & 7. \\
\hline & 9.0201 & -6.7043 & -0.6059 \\
\hline & 8.9111 & -5.6839 & -2 . \\
\hline & 4.9976 & -6.3241 & -1 . \\
\hline & 0.0046 & -7.4572 & \\
\hline & -0.7901 & -5.8823 & \\
\hline & 3.9887 & -5.1460 & -6 . \\
\hline & 2.1330 & 0.7039 & 4. \\
\hline & -4.3325 & 1.6509 & $1.2+2$ \\
\hline & 2.3412 & 6.8322 & \\
\hline & 1.0245 & 6.2 & -2 \\
\hline & 0.7646 & 5.0436 & -2 \\
\hline & 0.1421 & 7.0519 & -1 \\
\hline & -1.2264 & 6.6025 & \\
\hline & -1.8607 & 6.2558 & -3 \\
\hline & -1.6697 & 6.9751 & -4 \\
\hline & -2.6265 & 5.1450 & -3 \\
\hline & -3.2231 & 4.5970 & \\
\hline & -2.7468 & 3.1652 & -4 \\
\hline C & -1.2396 & 3.0054 & -4.828 \\
\hline$E$ & -0.8779 & 1.5201 & -4 \\
\hline & -0.7703 & 3. & \\
\hline & 3.1478 & 1.3169 & -3 \\
\hline C & 2.7087 & 2.5012 & -3.8 \\
\hline & 2.9373 & 2.7244 & -5.2 \\
\hline & 3.6080 & 1.7709 & -6 \\
\hline & 4.0581 & 0.5958 & -5.4 \\
\hline & 3.8352 & 0.3682 & -4.0 \\
\hline & 2.8937 & 1.1609 & -1 \\
\hline & 2.4618 & 2.1606 & -1 \\
\hline & 2.5083 & 7.8326 & -2 \\
\hline & 3.1467 & 6.1701 & -2 \\
\hline & -1.2514 & 5. & \\
\hline & -1.7938 & 7.4 & -1 \\
\hline & -3.0365 & 2.5439 & -3.7 \\
\hline & -3.3022 & 2.7747 & -5.4 \\
\hline & -0.7 & & \\
\hline & 0.2893 & 3.5 & -6 . \\
\hline & -1.3306 & 3.4854 & -6 . \\
\hline & -0.8933 & 4.8636 & - \\
\hline | & -1.3807 & 1.0462 & \\
\hline & -1.1770 & 0.9795 & \\
\hline & 0.1994 & 1.3845 & -5 \\
\hline & -2.9713 & 5.2730 & -5.1341 \\
\hline & 2.200 & 3.2483 & \\
\hline & 2.5939 & 3.6499 & -5.7 \\
\hline & 3.7849 & 1.9456 & $-7 . e$ \\
\hline & 4.5888 & -0.1485 & I \\
\hline H & 4.1872 & -0.5592 & -3.6 \\
\hline & -4.9661 & -2.9804 & 37 \\
\hline & 5154 & -2.4455 & 3.6 \\
\hline & -3.2820 & -1.2529 & (2.2 \\
\hline & -5.8869 & -2.2061 & \\
\hline & -5.6854 & -2.6449 & \\
\hline & -6.6237 & -3.4151 & \\
\hline
\end{tabular}




\begin{tabular}{|c|c|c|c|}
\hline 0 & -4.6554 & -2.3152 & 0.6696 \\
\hline $\mathrm{N}$ & -2.5260 & -3.3283 & 3.9765 \\
\hline $\mathrm{C}$ & -1.1600 & -2.8678 & 4.2393 \\
\hline $\mathrm{C}$ & -0.3614 & -2.5746 & 2.9587 \\
\hline 0 & -0.6976 & -3.0365 & 1.8627 \\
\hline $\mathrm{C}$ & -0.5250 & -4.0746 & 4.9610 \\
\hline C & -1.2043 & -5.2624 & 4.2663 \\
\hline $\mathrm{C}$ & -2.6527 & -4.7885 & 4.0809 \\
\hline $\mathrm{N}$ & 0.7490 & -1.8446 & 3.1768 \\
\hline $\mathrm{C}$ & 1.7583 & -1.6509 & 2.1597 \\
\hline $\mathrm{C}$ & 3.1116 & -2.2305 & 2.5884 \\
\hline 0 & 3.2306 & -3.3719 & 3.0253 \\
\hline $\mathrm{N}$ & 4.1341 & -1.3569 & 2.4042 \\
\hline $\mathrm{C}$ & 5.5310 & -1.6563 & 2.6521 \\
\hline $\mathrm{C}$ & 6.0726 & -1.1369 & 3.9964 \\
\hline 0 & 7.2821 & -1.2160 & 4.2230 \\
\hline C & 6.4290 & -1.0827 & 1.5500 \\
\hline 0 & 6.3116 & 0.3360 & 1.4433 \\
\hline $\mathrm{N}$ & 5.1949 & -0.5817 & 4.8516 \\
\hline $\mathrm{C}$ & 5.6873 & -0.0445 & 6.1061 \\
\hline $\mathrm{H}$ & -6.9316 & -2.3572 & 3.0287 \\
\hline $\mathrm{H}$ & -5.6499 & -1.1368 & 2.7967 \\
\hline $\mathrm{H}$ & -5.0135 & -4.0545 & 3.5107 \\
\hline $\mathrm{H}$ & -0.7761 & -4.0437 & 6.0264 \\
\hline $\mathrm{H}$ & 0.5616 & -4.0814 & 4.8614 \\
\hline $\mathrm{H}$ & -1.1426 & -6.1935 & 4.8352 \\
\hline $\mathrm{H}$ & -0.7452 & -5.4202 & 3.2867 \\
\hline $\mathrm{H}$ & -3.2752 & -5.0667 & 4.9426 \\
\hline $\mathrm{H}$ & -3.1084 & -5.2089 & 3.1793 \\
\hline $\mathrm{H}$ & -1.1893 & -1.9679 & 4.8587 \\
\hline $\mathrm{H}$ & 1.8604 & -0.5888 & 1.9288 \\
\hline $\mathrm{H}$ & 1.4320 & -2.1768 & 1.2607 \\
\hline $\mathrm{H}$ & 7.4590 & -1.2911 & 1.8308 \\
\hline $\mathrm{H}$ & 6.2258 & -1.5652 & 0.5913 \\
\hline $\mathrm{H}$ & 5.4508 & 0.5750 & 1.0337 \\
\hline $\mathrm{H}$ & 5.6364 & -2.7463 & 2.6645 \\
\hline $\mathrm{H}$ & 6.4791 & 0.6840 & 5.9110 \\
\hline $\mathrm{H}$ & 2.3673 & 6.8810 & -4.0860 \\
\hline $\mathrm{H}$ & -4.3167 & 4.5947 & -4.2115 \\
\hline $\mathrm{H}$ & -2.5516 & 4.5215 & -2.3024 \\
\hline $\mathrm{H}$ & 0.3048 & 8.0447 & -2.0182 \\
\hline $\mathrm{H}$ & 6.1083 & -0.8320 & 6.7426 \\
\hline $\mathrm{H}$ & 4.8523 & 0.4371 & 6.6176 \\
\hline $\mathrm{H}$ & 4.1877 & -0.6745 & 4.7313 \\
\hline $\mathrm{H}$ & 1.0389 & -1.5430 & 4.1214 \\
\hline $\mathrm{H}$ & -7.4850 & -3.7214 & 1.1834 \\
\hline $\mathrm{H}$ & -6.4765 & -3.6909 & -0.2267 \\
\hline $\mathrm{H}$ & 3.9145 & -0.4767 & 1.9512 \\
\hline $\mathrm{H}$ & -5.3197 & -2.8262 & 4.7310 \\
\hline $\mathrm{C}$ & -11.4379 & -4.5570 & 2.0068 \\
\hline $\mathrm{C}$ & -10.3373 & -4.2163 & 1.0247 \\
\hline 0 & -9.1696 & -4.5543 & 1.2404 \\
\hline C & -11.1756 & -3.7790 & 3.2995 \\
\hline $\mathrm{N}$ & -10.6962 & -3.4739 & -0.0461 \\
\hline C & -9.7134 & -2.9616 & -0.9827 \\
\hline $\mathrm{C}$ & -8.5320 & 7.3167 & 3.3499 \\
\hline $\mathrm{C}$ & -8.4186 & 5.9893 & 2.6134 \\
\hline C & -8.4117 & 6.1076 & 1.0915 \\
\hline $\mathrm{N}$ & -7.9288 & 4.9924 & 0.4669 \\
\hline 0 & -8.8514 & 7.0823 & 0.4887 \\
\hline $\mathrm{H}$ & -10.1800 & -4.0187 & 3.6795 \\
\hline $\mathrm{H}$ & -11.9118 & -4.0289 & 4.0685 \\
\hline $\mathrm{H}$ & -11.2224 & -2.6994 & 3.1244 \\
\hline $\mathrm{H}$ & -12.4299 & -4.3272 & 1.6023 \\
\hline $\mathrm{H}$ & -9.1080 & -2.1605 & -0.5453 \\
\hline $\mathrm{H}$ & -7.5308 & 5.4323 & 2.9202 \\
\hline $\mathrm{H}$ & -9.2751 & 5.3540 & 2.8711 \\
\hline $\mathrm{H}$ & -7.3673 & 4.3204 & 0.9910 \\
\hline $\mathrm{H}$ & -7.8097 & 5.0455 & -0.5338 \\
\hline $\mathrm{H}$ & -9.3958 & 7.8836 & 2.9928 \\
\hline $\mathrm{H}$ & -8.6351 & 7.1531 & 4.4271 \\
\hline $\mathrm{H}$ & -7.6457 & 7.9373 & 3.1841 \\
\hline $\mathrm{H}$ & -11.3947 & -5.6333 & 2.1981 \\
\hline $\mathrm{H}$ & -10.2256 & -2.5759 & -1.865 \\
\hline $\mathrm{H}$ & -9.0404 & -3.7661 & -1.2870 \\
\hline $\mathrm{H}$ & -11.6551 & -3.1757 & -0.1298 \\
\hline $\mathrm{C}$ & 3.9069 & 0.2974 & -1.0012 \\
\hline 0 & 3.8448 & 0.7392 & 0.3528 \\
\hline $\mathrm{C}$ & 3.7513 & -1.2122 & -1.0072 \\
\hline $\mathrm{H}$ & 2.7989 & -1.4755 & -0.5492 \\
\hline $\mathrm{H}$ & & & \\
\hline
\end{tabular}

$\begin{array}{llll}\mathrm{H} & 4.5474 & -1.6694 & -0.4203\end{array}$

TS3(AA)-R

$\begin{array}{ll}\text { C } & 1 \\ \text { C } & \\ \text { C } & \\ \text { C } & \\ \text { N } & \\ \text { C } & \end{array}$

\begin{tabular}{|c|c|c|}
\hline 34 & 513 & -0.4710 \\
\hline 9.960 & 3564 & -0.1962 \\
\hline & & \\
\hline 8. & 738 & 108 \\
\hline & & \\
\hline 9 & & 2 \\
\hline & & 1. \\
\hline 2. & I & 0.462 \\
\hline 0 & & -1.1 \\
\hline 9.7 & & \\
\hline 7.7824 & 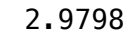 & -0.7 \\
\hline 9.3 & & \\
\hline 7.6109 & 1.30 & 1.1 \\
\hline 0 & & -4 \\
\hline-8 . & & -3.1 \\
\hline-7 . & 2. & -2.5 \\
\hline & & -3.3 \\
\hline-7 . & & -1.3 \\
\hline-5. & & -2.8 \\
\hline-6 . & . & -0.8 \\
\hline 94 & & -1 \\
\hline & & -1 . \\
\hline-9.5 & 1. & -3.8 \\
\hline & & -2 \\
\hline-8. & & -3 \\
\hline & & -4 \\
\hline-8. & & -0 . \\
\hline-4 & 6 & -3. \\
\hline & & \\
\hline-4 & 0 & -0.3 \\
\hline-0. & & -5 \\
\hline-1 . & & -4 \\
\hline-2 . & & -3.5 \\
\hline & & -2 . \\
\hline-3. & -5 & -4.2 \\
\hline-2 . & -4 & -1 . \\
\hline-4. & -4 & -3 \\
\hline-3.8 & -4 & -2 \\
\hline-4 & & -1 \\
\hline 0.1 & -5 & -4 \\
\hline-1. & -8 & -4.7 \\
\hline-0. & -7 & -3 \\
\hline-1.2 & -6 & -1 \\
\hline-3 . & & -5 \\
\hline-2 . & -4 & -0. \\
\hline-4. & -4 & -4 \\
\hline-4 . & -3 & -0 \\
\hline 4.8 & -4 & -5 \\
\hline 5. & -4 & -4. \\
\hline 6.2 & -5 & -4.1 \\
\hline 4. & -2 & -5 \\
\hline 5. & -4 & -6 \\
\hline 5.4 & -2 . & -4 \\
\hline 3. & -2 & -4 \\
\hline 4.2 & -2 . & -6.2 \\
\hline & -5 & -3 \\
\hline & -5 & -2 \\
\hline 2.8 & -5 & -1.5 \\
\hline & & -1 \\
\hline 1. & -4 & -0 . \\
\hline 3. & -3 & -0. \\
\hline & -4 & -3 \\
\hline & & -1 \\
\hline & -5 & -0 \\
\hline & -6 & -2 \\
\hline & -3 & -2 \\
\hline & -4 . & \\
\hline & -3 & -0. \\
\hline & & -0 \\
\hline 4.1908 & -3 & -1.5 \\
\hline & & \\
\hline & -2 & -0 . \\
\hline & & -1 \\
\hline & & -1. \\
\hline 7 & -3 & -1 \\
\hline
\end{tabular}

\begin{tabular}{|c|c|c|c|}
\hline & 7.2711 & -2.6307 & -2.3200 \\
\hline & 8.7492 & -2.5724 & -0.4238 \\
\hline & 768 & 2438 & 4478 \\
\hline & 687 & -1.1887 & -0.55 \\
\hline & 8.1792 & -0.5173 & -1.5666 \\
\hline & 9.7699 & -5.2894 & -0.3261 \\
\hline & 7.2 & -5 . & -0.2327 \\
\hline & & & \\
\hline & 6.6 & 122 & -3.0236 \\
\hline & & & \\
\hline & 6.8 & & - \\
\hline & & -0. & \\
\hline & 8.2 & & -1.6621 \\
\hline & & & \\
\hline & $-1.2<>$ & & \\
\hline & -1.3 & & -0 . \\
\hline & -0.2 & & -1.3763 \\
\hline & & & \\
\hline & -3.5 & & -0 . \\
\hline & -3.4921 & & 0. \\
\hline & 1.4615 & -0 . & -3 \\
\hline & & & \\
\hline & -4.7 & & -0 . \\
\hline & 0.2968 & -0. & -1.3106 \\
\hline & -0.5 & -1 . & -1 . \\
\hline & -1. & & \\
\hline & -0.1 & -1 . & -2 \\
\hline & -0.6210 & -2 & -3.8462 \\
\hline & -0. & & \\
\hline & 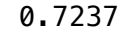 & & -1 \\
\hline & -0.3 & & -1 \\
\hline & -0.1651 & -0. & 0. \\
\hline & & & \\
\hline & -5 & & \\
\hline & -4.5 & & -0. \\
\hline & -5 & & -0. \\
\hline & & & \\
\hline & -1 & -1 & -0 . \\
\hline & -2.2220 & -2 & -1 \\
\hline & -2 & -0 & -1 \\
\hline & -0 . & & \\
\hline & -0.3 & -2 & -4 \\
\hline & 1.8486 & -3 & 7. \\
\hline & 1.1 & -1 & \\
\hline & & & \\
\hline 0 & 2.0 & -1 & \\
\hline 0 & 2.2 & & \\
\hline$C$ & -8. & & \\
\hline 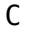 & -7.2 & & \\
\hline$C$ & -6.3 & & \\
\hline 0 & -6.3 & & 33 \\
\hline$J$ & -5. & & \\
\hline & -8. & & \\
\hline & -7.6 & & 38 \\
\hline & -7.8 & & \\
\hline & & & \\
\hline & -0 . & & \\
\hline C & -0.6 & & \\
\hline & 0. & & \\
\hline & & & \\
\hline & & & \\
\hline & 2.8 & & \\
\hline & 2.4 & & \\
\hline 1 & & & \\
\hline & -0 & & \\
\hline & -1.2 & & \\
\hline $\mathrm{H}$ & & & \\
\hline & & & \\
\hline & & & \\
\hline & 5. & & \\
\hline$C$ & & & \\
\hline & & & \\
\hline & & & \\
\hline & & & \\
\hline 11 & & & \\
\hline & & & \\
\hline & & & \\
\hline & & & \\
\hline & & & \\
\hline & & & \\
\hline & 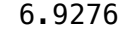 & & \\
\hline
\end{tabular}




\begin{tabular}{|c|c|c|c|c|c|c|c|c|c|c|c|}
\hline $\mathrm{H}$ & 4.3853 & 3.8853 & 2.0819 & C & 1.5490 & -1.6254 & 2.1700 & C & 8.6980 & 2.6995 & -0.178 \\
\hline $\mathrm{H}$ & 5.8215 & 3.1085 & 1.4142 & C & 2.8761 & -2.3089 & 2.4996 & $\mathrm{~N}$ & 10.0914 & 3.5470 & 1.383 \\
\hline $\mathrm{H}$ & 5.5785 & 4.8345 & 1.1758 & 0 & 2.9547 & -3.5041 & 2.7785 & C & 9.4609 & 2.4929 & 1.872 \\
\hline $\mathrm{H}$ & -1.7124 & -2.8591 & -3.8212 & $\mathrm{~N}$ & 3.9253 & -1.4587 & 2.4197 & $\mathrm{~N}$ & 8.6118 & 1.9361 & 0.967 \\
\hline $\mathrm{H}$ & -8.9006 & 2.9018 & 3.4473 & C & 5.3136 & -1.8151 & 2.6142 & $\mathrm{H}$ & 12.1118 & 4.8480 & -0.004 \\
\hline $\mathrm{H}$ & 11.7432 & 4.2410 & -1.1565 & C & 5.8951 & -1.3540 & 3.9676 & $\mathrm{H}$ & 9.5922 & 4.7338 & -1.764 \\
\hline $\mathrm{H}$ & 11.7982 & 5.9993 & -0.9131 & 0 & 7.1130 & -1.3997 & 4.1457 & H & 9.7909 & 5.7642 & -0.353 \\
\hline $\mathrm{H}$ & -10.2819 & 3.0440 & -4.6401 & C & 6.1773 & -1.2144 & 1.5020 & $\mathrm{H}$ & 8.0979 & 2.4881 & -1.05 \\
\hline $\mathrm{H}$ & -8.8277 & 2.1175 & -5.0543 & 0 & 6.1113 & 0.2129 & 1.5161 & H & 9.5727 & 2.0950 & 2.871 \\
\hline $\mathrm{H}$ & -0.7003 & 2.7132 & 5.7112 & $\mathrm{~N}$ & 5.0240 & -0.8928 & 4.8876 & $\mathrm{H}$ & 7.9457 & 1.1738 & 1.146 \\
\hline $\mathrm{H}$ & -1.7088 & 1.2709 & 5.9392 & C & 5.5175 & -0.4350 & 6.1762 & C & -9.4797 & 2.4615 & -4.132 \\
\hline $\mathrm{H}$ & 6.5552 & 7.0796 & 2.8276 & $\mathrm{H}$ & -6.9842 & -2.6419 & 2.7801 & C & -8.4917 & 3.2201 & -3.226 \\
\hline $\mathrm{H}$ & 5.2192 & 7.6004 & 3.8664 & $\mathrm{H}$ & -5.6918 & -1.4086 & 2.8257 & C & -7.4329 & 2.3083 & -2.649 \\
\hline $\mathrm{H}$ & 0.0960 & -2.0326 & 6.9123 & $\mathrm{H}$ & -5.0532 & -4.4028 & 3.0113 & C & -6.3100 & 1.9423 & -3.407 \\
\hline $\mathrm{H}$ & 0.9899 & -1.5953 & 8.3776 & $\mathrm{H}$ & -0.7481 & -4.9091 & 5.3894 & C & -7.5695 & 1.7463 & -1.374 \\
\hline $\mathrm{H}$ & 2.0022 & -3.5297 & 6.2031 & $\mathrm{H}$ & 0.5399 & -4.5475 & 4.2184 & C & -5.3759 & 1.0311 & -2.925 \\
\hline $\mathrm{H}$ & 1.3045 & -4.0632 & 7.7511 & $\mathrm{H}$ & -1.0883 & -6.6593 & 3.6705 & C & -6.6439 & 0.8308 & -0.873 \\
\hline $\mathrm{H}$ & 2.8361 & -3.1763 & 7.7173 & $\mathrm{H}$ & -0.7495 & -5.4908 & 2.3808 & C & -5.5469 & 0.4594 & -1.658 \\
\hline $\mathrm{H}$ & 8.9353 & -6.6675 & -1.0556 & $\mathrm{H}$ & -3.2757 & -5.7317 & 4.0508 & 0 & -4.5992 & -0.4170 & -1.243 \\
\hline $\mathrm{H}$ & 9.6699 & -5.4277 & -2.0864 & $\mathrm{H}$ & -3.1036 & -5.3063 & 2.3342 & $\mathrm{H}$ & -9.9911 & 1.6742 & -3.569 \\
\hline $\mathrm{H}$ & 4.5003 & -6.6840 & -2.4374 & $\mathrm{H}$ & -1.2862 & -2.6064 & 4.8546 & $\mathrm{H}$ & -9.0431 & 3.7063 & -2.413 \\
\hline $\mathrm{H}$ & 0.1942 & -7.2519 & -5.9003 & $\mathrm{H}$ & 1.6783 & -0.5448 & 2.1081 & $\mathrm{H}$ & -8.0181 & 4.0216 & -3.806 \\
\hline $\mathrm{H}$ & -1.1030 & -6.0719 & -6.1525 & $\mathrm{H}$ & 1.2174 & -1.9953 & 1.1996 & H & -6.1677 & 2.3735 & -4.395 \\
\hline $\mathrm{H}$ & 3.9389 & -4.6232 & -6.2781 & $\mathrm{H}$ & 7.2129 & -1.4831 & 1.6917 & $\mathrm{H}$ & -8.4068 & 2.0453 & -0.749 \\
\hline $\mathrm{H}$ & 2.0085 & 0.4289 & 4.6380 & $\mathrm{H}$ & 5.8784 & -1.6098 & 0.5239 & $\mathrm{H}$ & -4.5123 & 0.7432 & -3.514 \\
\hline $\mathrm{H}$ & -4.3799 & 1.5014 & 1.3207 & $\mathrm{H}$ & 5.2577 & 0.4919 & 1.1207 & $\mathrm{H}$ & -6.7284 & 0.4617 & 0.143 \\
\hline C & 2.2155 & 6.9049 & -2.5710 & $\mathrm{H}$ & 5.3784 & -2.9076 & 2.5812 & $\mathrm{H}$ & -4.8297 & -0.8606 & -0.403 \\
\hline C & 0.8235 & 6.3883 & -2.3353 & $\mathrm{H}$ & 6.3019 & 0.3115 & 6.0267 & C & -0.6807 & -6.7431 & -4.946 \\
\hline 0 & 0.5002 & 5.2552 & -2.7048 & $\mathrm{H}$ & 2.3803 & 6.9846 & -3.6492 & C & -2.0153 & -7.3375 & -4.458 \\
\hline $\mathrm{N}$ & -0.0524 & 7.1984 & -1.7008 & $\mathrm{H}$ & -4.3836 & 4.6389 & -4.0317 & C & -2.8890 & -6.3431 & -3.723 \\
\hline C & -1.4404 & 6.7902 & -1.5655 & $\mathrm{H}$ & -2.6625 & 4.6438 & -2.0981 & C & -2.8752 & -6.2547 & -2.326 \\
\hline C & -2.0300 & 6.4065 & -2.9363 & $\mathrm{H}$ & 0.1936 & 8.1637 & -1.5528 & C & -3.7189 & -5.4550 & $-4.42]$ \\
\hline 0 & -1.8709 & 7.1321 & -3.9135 & $\mathrm{H}$ & 5.9472 & -1.2594 & 6.7581 & C & -3.6430 & -5.3110 & -1.644 \\
\hline $\mathrm{N}$ & -2.7278 & 5.2512 & -2.9179 & $\mathrm{H}$ & 4.6801 & 0.0037 & 6.7207 & C & -4.4889 & -4.5035 & -3.757 \\
\hline C & -3.2885 & 4.6652 & -4.1194 & $\mathrm{H}$ & 4.0174 & -1.0137 & 4.7838 & C & -4.4489 & -4.4187 & -2.361 \\
\hline C & -2.7844 & 3.2379 & -4.3635 & $\mathrm{H}$ & 0.8520 & -1.8375 & 4.1240 & 0 & -5.2157 & -3.4662 & -1.753 \\
\hline C & -1.2989 & 3.1037 & -4.7306 & $\mathrm{H}$ & -7.4624 & -3.5770 & 0.7129 & $\mathrm{H}$ & -0.0898 & -6.3733 & -4.102 \\
\hline C & -0.8893 & 1.6266 & -4.7272 & $\mathrm{H}$ & -6.3673 & -3.3762 & -0.6247 & $\mathrm{H}$ & -2.5635 & -7.7360 & -5.32 \\
\hline C & -0.9817 & 3.7687 & -6.0749 & $\mathrm{H}$ & 3.7272 & -0.5161 & 2.1134 & $\mathrm{H}$ & -1.8081 & -8.1913 & -3.802 \\
\hline C & 3.0115 & 1.7657 & -3.2704 & $\mathrm{H}$ & -5.4628 & -3.4516 & 4.4303 & $\mathrm{H}$ & -2.2506 & -6.9353 & -1.756 \\
\hline C & 2.4757 & 2.9258 & -3.8345 & C & -11.2331 & -5.0853 & 1.5683 & $\mathrm{H}$ & -3.7539 & -5.5011 & -5.507 \\
\hline C & 2.6710 & 3.2059 & -5.1870 & C & -10.1762 & -4.5113 & 0.6504 & $\mathrm{H}$ & -3.6074 & -5.2490 & -0.561 \\
\hline C & 3.4029 & 2.3319 & -5.9915 & 0 & -8.9813 & -4.5670 & 0.9549 & H & -5.1135 & -3.8028 & -4.300 \\
\hline C & 3.9572 & 1.1794 & -5.4299 & C & -11.2322 & -4.2576 & 2.8568 & $\mathrm{H}$ & -4.7902 & -3.1160 & -0.933 \\
\hline C & 3.7690 & 0.9029 & -4.0774 & $\mathrm{~N}$ & -10.6177 & -3.8775 & -0.4579 & C & 4.6216 & -4.5590 & -5.57 \\
\hline C & 2.8021 & 1.5172 & -1.7852 & C & -9.7108 & -3.1717 & -1.3443 & C & 4.4091 & -4.4360 & -4.078 \\
\hline 0 & 2.2605 & 2.4293 & -1.0851 & C & -8.7707 & 6.8555 & 3.5936 & 0 & 4.6464 & -3.4049 & -3.453 \\
\hline $\mathrm{H}$ & 2.4076 & 7.8756 & -2.1071 & C & -8.6345 & 5.5607 & 2.8076 & C & 4.6505 & -3.1962 & -6.264 \\
\hline $\mathrm{H}$ & 2.9248 & 6.1712 & -2.1804 & C & -8.6290 & 5.7362 & 1.2918 & $\mathrm{H}$ & 5.5756 & -5.0815 & -5.725 \\
\hline $\mathrm{H}$ & -1.5221 & 5.9634 & -0.8528 & $\mathrm{~N}$ & -8.0955 & 4.6656 & 0.6311 & $\mathrm{H}$ & 5.4305 & -2.5724 & -5.822 \\
\hline $\mathrm{H}$ & -2.0048 & 7.6369 & -1.1682 & 0 & -9.1107 & 6.7107 & 0.7219 & $\mathrm{H}$ & 3.7012 & -2.6696 & -6.132 \\
\hline $\mathrm{H}$ & -2.9907 & 2.6443 & -3.4652 & $\mathrm{H}$ & -10.2346 & -4.2659 & 3.3010 & $\mathrm{H}$ & 4.8458 & -3.3024 & -7.335 \\
\hline $\mathrm{H}$ & -3.3934 & 2.7969 & -5.1663 & $\mathrm{H}$ & -11.9421 & -4.6591 & 3.5853 & $\mathrm{~N}$ & 3.9826 & -5.5857 & -3.492 \\
\hline $\mathrm{H}$ & -0.7088 & 3.6179 & -3.9679 & $\mathrm{H}$ & -11.5061 & -3.2174 & 2.6546 & C & 4.0298 & -5.7934 & -2.061 \\
\hline $\mathrm{H}$ & 0.0604 & 3.5894 & -6.3553 & $\mathrm{H}$ & -12.2234 & -5.0859 & 1.0994 & C & 2.6810 & -6.1426 & -1.429 \\
\hline $\mathrm{H}$ & -1.6159 & 3.3646 & -6.8748 & $\mathrm{H}$ & -9.1638 & -2.3878 & -0.8120 & C & 1.6272 & -5.0251 & -1.464 \\
\hline $\mathrm{H}$ & -1.1326 & 4.8514 & -6.0306 & H & -7.7378 & 5.0080 & 3.0950 & C & 0.3176 & -5.5215 & -0.844 \\
\hline $\mathrm{H}$ & -1.4533 & 1.0586 & -5.4787 & $\mathrm{H}$ & -9.4819 & 4.9034 & 3.0389 & C & 2.1142 & -3.7593 & -0.753 \\
\hline $\mathrm{H}$ & -1.0797 & 1.1669 & -3.7516 & $\mathrm{H}$ & -7.5016 & 4.0082 & 1.1362 & $\mathrm{H}$ & 3.8334 & -6.3894 & -4.084 \\
\hline $\mathrm{H}$ & 0.1758 & 1.5126 & -4.9454 & $\mathrm{H}$ & -7.9775 & 4.7545 & -0.3673 & H & 4.4302 & -4.8759 & -1.628 \\
\hline $\mathrm{H}$ & -3.0450 & 5.3333 & -4.9475 & $\mathrm{H}$ & -9.6416 & 7.4232 & 3.2554 & $\mathrm{H}$ & 2.8684 & -6.4196 & -0.383 \\
\hline $\mathrm{H}$ & 1.9181 & 3.6141 & -3.2118 & $\mathrm{H}$ & -8.8755 & 6.6491 & 4.6632 & $\mathrm{H}$ & 2.2774 & -7.0426 & -1.916 \\
\hline $\mathrm{H}$ & 2.2485 & 4.1137 & -5.6067 & $\mathrm{H}$ & -7.8930 & 7.4954 & 3.4567 & $\mathrm{H}$ & 1.4382 & -4.7733 & -2.517 \\
\hline $\mathrm{H}$ & 3.5519 & 2.5509 & -7.0447 & $\mathrm{H}$ & -10.9633 & -6.1232 & 1.7845 & $\mathrm{H}$ & 0.4519 & -5.7284 & 0.223 \\
\hline $\mathrm{H}$ & 4.5444 & 0.5002 & -6.0409 & $\mathrm{H}$ & -10.2854 & -2.7148 & -2.1506 & $\mathrm{H}$ & -0.4720 & -4.7725 & -0.934 \\
\hline $\mathrm{H}$ & 4.2197 & 0.0114 & -3.6526 & $\mathrm{H}$ & -8.9796 & -3.8600 & -1.7789 & H & -0.0290 & -6.4414 & -1.328 \\
\hline C & -5.0442 & -3.3893 & 3.4187 & $\mathrm{H}$ & -11.6081 & -3.8461 & -0.6390 & $\mathrm{H}$ & 2.9778 & -3.3236 & -1.258 \\
\hline C & -3.6198 & -2.8359 & 3.5886 & C & 3.9833 & 0.8262 & -1.0712 & $\mathrm{H}$ & 2.3841 & -3.9736 & 0.287 \\
\hline 0 & -3.4191 & -1.6321 & 3.7368 & $\mathrm{H}$ & 4.1445 & -0.1934 & -1.4299 & $\mathrm{H}$ & 1.3220 & -3.0079 & -0.747 \\
\hline C & -5.9270 & -2.4500 & 2.5755 & 0 & 3.6700 & 0.7261 & 0.3146 & C & 9.0038 & -5.7971 & -0.967 \\
\hline C & -5.6442 & -2.6372 & 1.1051 & C & 5.2534 & 1.6653 & -1.2671 & C & 8.3769 & -4.7675 & -0.013 \\
\hline $\mathrm{N}$ & -6.6041 & -3.1736 & 0.3416 & $\mathrm{H}$ & 6.0721 & 1.2069 & -0.7143 & C & 8.2487 & -3.3915 & -0.628 \\
\hline 0 & -4.5319 & -2.3076 & 0.6230 & $\mathrm{H}$ & 5.5402 & 1.7291 & -2.3183 & C & 7.2579 & -3.1324 & -1.583 \\
\hline $\mathrm{N}$ & -2.6145 & -3.7468 & 3.6902 & $\mathrm{H}$ & 5.0828 & 2.6766 & -0.8885 & C & 9.1103 & -2.3495 & -0.265 \\
\hline C & -1.2624 & -3.3079 & 4.0169 & & & & & C & 7.1273 & -1.8689 & -2.158 \\
\hline C & -0.5641 & -2.6551 & 2.8193 & EP( & AA)-S & & & C & 8.9793 & -1.0786 & -0.828 \\
\hline 0 & -0.9576 & -2.8392 & 1.6615 & & & & & C & 7.9854 & -0.8355 & -1.777 \\
\hline C & -0.5408 & -4.6296 & 4.3513 & C & 11.6193 & 4.8075 & -0.97 & $\mathrm{H}$ & 10.0110 & -5.4886 & -1.264 \\
\hline C & -1.1960 & -5.6139 & 3.3708 & c & 10.0947 & 4.8040 & -0.7917 & $\mathrm{H}$ & 8.9772 & -4.7039 & 0.901 \\
\hline C & -2.6680 & -5.1700 & 3.3287 & C & 9.6166 & 3.6902 & 0.0911 & $\mathrm{H}$ & 7.3841 & -5.1205 & 0.292 \\
\hline IV & 0.5453 & -1.9695 & 3.1479 & & & & & $\mathrm{H}$ & 6.5656 & -3.9132 & -1.882 \\
\hline
\end{tabular}




\begin{tabular}{|c|c|c|c|}
\hline $\mathrm{H}$ & 9.8763 & -2.5286 & 0.4844 \\
\hline $\mathrm{H}$ & 6.3464 & -1.7148 & -2.8943 \\
\hline $\mathrm{H}$ & 9.6365 & -0.2745 & -0.5135 \\
\hline $\mathrm{H}$ & 7.8833 & 0.1533 & -2.2152 \\
\hline C & -2.6860 & 0.8795 & 0.6523 \\
\hline C & -1.5177 & 1.0086 & -0.0480 \\
\hline $\mathrm{C}$ & -1.3309 & 2.2377 & -0.7702 \\
\hline $\mathrm{N}$ & -0.1831 & 2.5451 & -1.3615 \\
\hline $\mathrm{N}$ & -2.3455 & 3.1453 & -0.8411 \\
\hline C & -3.4757 & 2.9173 & -0.1920 \\
\hline $\mathrm{N}$ & -3.6518 & 1.8363 & 0.5965 \\
\hline S & 1.0407 & -0.9628 & -3.4641 \\
\hline 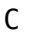 & 0.9028 & -0.0373 & -2.0174 \\
\hline C & -4.6136 & 3.8656 & -0.3683 \\
\hline 10 & -0.0945 & -0.5990 & -1.3080 \\
\hline C & -0.7831 & -1.7090 & -1.8452 \\
\hline C & -1.9303 & -2.3192 & -1.1201 \\
\hline C & -0.2720 & -2.0591 & -3.0548 \\
\hline C & -0.7042 & -3.1648 & -3.9639 \\
\hline C & -0.4309 & -0.0254 & 0.0160 \\
\hline$H$ & 0.5575 & 1.8385 & -1.4867 \\
\hline $\mathrm{H}$ & -0.0452 & 3.4517 & -1.8073 \\
\hline $\mathrm{H}$ & -0.7081 & -0.8359 & 0.6892 \\
\hline $\mathrm{H}$ & 0.4868 & 0.4322 & 0.3883 \\
\hline $\mathrm{H}$ & -5.1971 & 3.9388 & 0.5526 \\
\hline $\mathrm{H}$ & -4.2403 & 4.8386 & -0.6879 \\
\hline $\mathrm{H}$ & -5.2762 & 3.4746 & -1.1492 \\
\hline $\mathrm{H}$ & -2.8980 & 0.0279 & 1.2940 \\
\hline $\mathrm{H}$ & -1.6808 & -2.5766 & -0.0885 \\
\hline $\mathrm{H}$ & -2.2356 & -3.2274 & -1.6343 \\
\hline $\mathrm{H}$ & -2.7786 & -1.6331 & -1.1185 \\
\hline $\mathrm{H}$ & 0.1545 & -3.7466 & -4.3138 \\
\hline $\mathrm{H}$ & -1.2233 & -2.7778 & -4.8484 \\
\hline C & 1.9787 & -2.7798 & 7.4302 \\
\hline C & 1.2173 & -1.4549 & 7.4349 \\
\hline C & 1.9710 & -0.4118 & 6.6076 \\
\hline 0 & 2.1406 & -0.7270 & 5.3598 \\
\hline 0 & 2.3758 & 0.6327 & 7.1340 \\
\hline C & -8.0443 & 3.1016 & 4.3022 \\
\hline C & -7.2268 & 1.9139 & 3.7927 \\
\hline $\mathrm{C}$ & -6.3631 & 2.2366 & 2.5684 \\
\hline 0 & -6.3723 & 3.4080 & 2.1219 \\
\hline 0 & -5.6704 & 1.2692 & 2.0995 \\
\hline $\mathrm{H}$ & -8.6117 & 2.8284 & 5.1994 \\
\hline $\mathrm{H}$ & -7.3948 & 3.9430 & 4.5490 \\
\hline $\mathrm{H}$ & -7.8747 & 1.0674 & 3.5307 \\
\hline $\mathrm{H}$ & -6.5501 & 1.5338 & 4.5677 \\
\hline$C$ & -0.5437 & 2.0586 & 5.4933 \\
\hline C & -0.4962 & 1.6275 & 4.0133 \\
\hline$C$ & 0.8194 & 1.8746 & 3.3362 \\
\hline$C$ & 1.1631 & 2.4296 & 2.1168 \\
\hline $\mathrm{N}$ & 2.0161 & 1.4750 & 3.8937 \\
\hline$C$ & 3.0035 & 1.7708 & 3.0185 \\
\hline $\mathrm{N}$ & 2.5314 & 2.3533 & 1.9238 \\
\hline $\mathrm{H}$ & 0.2508 & 1.5925 & 6.0808 \\
\hline $\mathrm{H}$ & -0.7454 & 0.5608 & 3.9420 \\
\hline $\mathrm{H}$ & -1.2756 & 2.1492 & 3.4464 \\
\hline $\mathrm{H}$ & 0.5209 & 2.8880 & 1.3790 \\
\hline $\mathrm{H}$ & 4.0394 & 1.5355 & 3.2113 \\
\hline $\mathrm{H}$ & 3.6508 & 1.7382 & 0.5883 \\
\hline C & 5.8449 & 6.9106 & 2.7213 \\
\hline$C$ & 5.8697 & 5.6051 & 3.5208 \\
\hline $\mathrm{C}$ & 6.4049 & 4.3774 & 2.7598 \\
\hline $\mathrm{C}$ & 6.4165 & 3.1614 & 3.6977 \\
\hline C & 5.5962 & 4.0904 & 1.4883 \\
\hline $\mathrm{H}$ & 5.1534 & 6.8513 & 1.8754 \\
\hline $\mathrm{H}$ & 4.8531 & 5.3783 & 3.8717 \\
\hline $\mathrm{H}$ & 6.4780 & 5.7489 & 4.4240 \\
\hline $\mathrm{H}$ & 7.4430 & 4.5837 & 2.4614 \\
\hline $\mathrm{H}$ & 5.4212 & 2.9940 & 4.1239 \\
\hline $\mathrm{H}$ & 6.7137 & 2.2460 & 3.1791 \\
\hline $\mathrm{H}$ & 7.1064 & 3.3151 & 4.5355 \\
\hline $\mathrm{H}$ & 4.5332 & 3.9485 & 1.7084 \\
\hline $\mathrm{H}$ & 5.9528 & 3.1808 & 0.9992 \\
\hline $\mathrm{H}$ & 5.6857 & 4.9042 & 0.7614 \\
\hline $\mathrm{H}$ & -1.3853 & -3.8503 & -3.4570 \\
\hline $\mathrm{H}$ & -8.7496 & 3.4387 & 3.5419 \\
\hline$\Pi$ & 11.9514 & 3.8930 & -1.4823 \\
\hline $\mathrm{H}$ & 11.9472 & 5.6665 & -1.5754 \\
\hline 11 & -10.2373 & 3.1344 & -4.5483 \\
\hline $\mathrm{H}$ & -8.9518 & 1.9831 & -4.9637 \\
\hline
\end{tabular}

\begin{tabular}{|c|c|c|c|}
\hline & -0.4212 & 1427 & \\
\hline & -1.5080 & 7848 & \\
\hline & 383 & 465 & \\
\hline & & 520 & \\
\hline & 28 & -1.5967 & \\
\hline & 311 & & \\
\hline & 740 & & \\
\hline & & & \\
\hline & 176 & -2 & \\
\hline & & & \\
\hline & 61 & -5.8946 & \\
\hline & & -6 & \\
\hline & -0. & & \\
\hline & -0. & -5 & \\
\hline & & & \\
\hline & & & \\
\hline & -4.5 & & \\
\hline & 2. & & \\
\hline & 1. & & \\
\hline & 0. & & -2 \\
\hline & & & \\
\hline & -1. & & \\
\hline & -1 & & \\
\hline & -1. & & \\
\hline & -2 & & \\
\hline & -3 & 4. & -4 \\
\hline & -2 & & -4 \\
\hline & -1 & & \\
\hline & -0. & & \\
\hline & -0. & & \\
\hline & 3. & & \\
\hline & 2. & & \\
\hline & 2.8 & & -5 \\
\hline & & & \\
\hline C & & & \\
\hline C & & & -4 \\
\hline & & & \\
\hline & & & \\
\hline & 2. & & -2 \\
\hline & 3. & & -2 \\
\hline & -1 & & \\
\hline & -1 & & \\
\hline 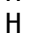 & -2. & & \\
\hline 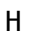 & -3 & & \\
\hline & -0. & & -4 \\
\hline & 0.2 & & -6 \\
\hline & -1 & & \\
\hline & -0. & & \\
\hline & -1 & & \\
\hline $\mathrm{H}$ & -1 & & -4 \\
\hline & 332 & & -5 \\
\hline $\mathrm{H}$ & -3.0393 & & \\
\hline & 2.2 & 3. & -3 \\
\hline & 2. & & \\
\hline & 3. & & -7 \\
\hline - & 4. & -0 & -5 \\
\hline $\mathrm{H}$ & 4. & 8 & \\
\hline$C$ & -4 & -2 & \\
\hline$C$ & -3.5 & -2 & \\
\hline 0 & -3 & -1 & \\
\hline C & -5 & -2 . & \\
\hline$C$ & -5 . & -2 & \\
\hline & -6 . & -3 & \\
\hline 0 & -4 & -2 & \\
\hline & -2 & -3 & \\
\hline & -1 & -2 & \\
\hline C & -0 . & -2 & \\
\hline 0 & -0 . & -2 . & \\
\hline$C$ & -0. & -4 & \\
\hline$C$ & -1 & -5 . & \\
\hline $\mathrm{C}$ & -2. & -4 & \\
\hline & 0. & -1. & \\
\hline 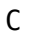 & & -1 & \\
\hline & & -2. & \\
\hline 作 & & -3 & \\
\hline & & -1 & \\
\hline $\mathrm{C}$ & & -1.7 & \\
\hline 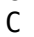 & 111 & -1.2 & \\
\hline & & -1.4878 & \\
\hline & & -1 & \\
\hline & & & \\
\hline
\end{tabular}

\begin{tabular}{|c|c|c|c|}
\hline 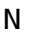 & 706 & -0.6287 & \\
\hline 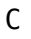 & 876 & 14 & \\
\hline & 756 & 3738 & \\
\hline & 087 & -1.1445 & \\
\hline & -5.0260 & -4.048 & \\
\hline & -0.7286 & -4.05 & \\
\hline & 0.5836 & -3 & \\
\hline & & & \\
\hline & -0. & -5 & \\
\hline & -3.2 & -5 & \\
\hline & -3 & -5. & \\
\hline & -1.2 & -1 & \\
\hline & 1.8340 & -0. & \\
\hline & 1.3537 & -1 & \\
\hline & & -1 & \\
\hline & 6.1827 & -1 & \\
\hline & 5.7478 & & \\
\hline & 5. & -2 . & \\
\hline & 6.50 & 0. & \\
\hline & 2.3 & & \\
\hline & -4.3 & 4. & -4 \\
\hline & -2 & & -2 \\
\hline & 0. & & -2 \\
\hline & 6. & -0. & \\
\hline & 4. & & \\
\hline & 4.7 & -0. & 4. \\
\hline & 0. & -1 & \\
\hline & -7 & -3 & \\
\hline & -6. & -3.7 & -0 . \\
\hline & 3. & -0. & \\
\hline $\mathrm{H}$ & -5.3586 & -2 & \\
\hline$C$ & -11. & -4 & \\
\hline C & -10. & -4 & \\
\hline 0 & -9.2577 & -4 & \\
\hline C & -11. & -3 & \\
\hline J & -10.7 & -3 & \\
\hline C & -9 & -2 . & -0 \\
\hline . & -8.2 & 7. & \\
\hline 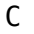 & -8 & & \\
\hline C & -8 & 6. & \\
\hline & -7. & & \\
\hline 0 & -8 & & \\
\hline $\mathrm{H}$ & -10 & -3 & \\
\hline H & -11.9549 & -3 & \\
\hline 1 & -11. & -2 . & \\
\hline H & -12 & -4 & \\
\hline$H$ & -9 . & -2 . & -0. \\
\hline 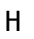 & -7 & & \\
\hline $\mathrm{H}$ & -9. & & \\
\hline $\mathrm{H}$ & -7 . & & \\
\hline $\mathrm{H}$ & -7.8 & & -0 . \\
\hline $\mathrm{H}$ & -9.3 & & \\
\hline 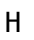 & -8 & & \\
\hline $\mathrm{H}$ & -7 & & \\
\hline 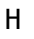 & -11. & -5 . & \\
\hline $\mathrm{H}$ & -10.2725 & -2 & -1 . \\
\hline $\mathrm{H}$ & -9.1 & -3 & -1 . \\
\hline 11 & -11.7071 & -3.0855 & -0 \\
\hline$C$ & 4.3462 & 0. & -0 . \\
\hline 0 & 4.3495 & & \\
\hline C & 3.8178 & -0.7858 & -1 . \\
\hline $\mathrm{H}$ & 2.8 & -0. & -0 . \\
\hline 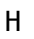 & 3.7855 & -1 & -2 \\
\hline & & -1 & \\
\hline & 5.3877 & 0.6653 & -1 . \\
\hline
\end{tabular}

EP(AA)-R

$\begin{array}{rrrr}\text { C } & 11.5373 & 4.8272 & -0.6270 \\ \mathrm{C} & 10.1201 & 4.9953 & -0.0571 \\ \mathrm{C} & 9.5972 & 3.7720 & 0.6353 \\ \mathrm{C} & 8.5821 & 2.9315 & 0.2366 \\ \mathrm{~N} & 10.1123 & 3.3406 & 1.8454 \\ \mathrm{C} & 9.4077 & 2.2676 & 2.1629 \\ \mathrm{~N} & 8.4728 & 1.9683 & 1.2186 \\ \mathrm{H} & 12.2305 & 4.5419 & 0.1695 \\ \mathrm{H} & 9.4277 & 5.2719 & -0.8614 \\ \mathrm{H} & 10.1205 & 5.8286 & 0.6566 \\ \mathrm{H} & 7.9382 & 2.9442 & -0.6266 \\ \mathrm{H} & 9.5196 & 1.6769 & 3.0614\end{array}$




\begin{tabular}{|c|c|c|c|}
\hline $\mathrm{H}$ & 7.7340 & 1.2611 & 1.2956 \\
\hline C & -9.5051 & 2.5122 & -4.1598 \\
\hline C & -8.6113 & 3.2753 & -3.1653 \\
\hline C & -7.5367 & 2.3809 & -2.5937 \\
\hline C & -6.3994 & 2.0641 & -3.3512 \\
\hline C & -7.6820 & 1.7670 & -1 \\
\hline C & -5.4621 & 1.1439 & -2.8973 \\
\hline C & -6.7513 & 0.8420 & -0.8695 \\
\hline C & -5.6444 & 0.5154 & -1.6592 \\
\hline 0 & -4.6969 & -0.3781 & -1.2867 \\
\hline $\mathrm{H}$ & -9.9942 & 1.6666 & -3.6654 \\
\hline H & -9.2264 & 3.6833 & -2.3551 \\
\hline 卜 & -8.1557 & 4.1325 & -3.6765 \\
\hline H & -6.2524 & 2.5370 & -4.3195 \\
\hline $\mathrm{H}$ & -8.5334 & 2.0269 & -0.7204 \\
\hline $\mathrm{H}$ & -4.5884 & 0.8894 & -3.4866 \\
\hline $\mathrm{H}$ & -6.8440 & 0.4218 & 0.1260 \\
\hline $\mathrm{H}$ & -4.9002 & -0.8515 & -0.4568 \\
\hline C & -0.6362 & -6.5885 & -5.3115 \\
\hline C & -1.5232 & -7.3164 & -4.2876 \\
\hline C & -2.4621 & -6.3794 & -3.5619 \\
\hline C & -2.2533 & -6.0094 & -2.2293 \\
\hline C & -3.5539 & -5.8109 & -4.2328 \\
\hline C & -3.0863 & -5.0943 & -1.5837 \\
\hline C & -4.3870 & -4.8879 & -3.6117 \\
\hline C & -4.1492 & -4.5113 & -2.2845 \\
\hline 0 & -4.9634 & -3.5628 & -1.7479 \\
\hline $\mathrm{H}$ & -0.0059 & -5.8465 & -4.8107 \\
\hline $\mathrm{H}$ & -2.1009 & -8.0930 & -4.8052 \\
\hline $\mathrm{H}$ & -0.8886 & -7.8358 & -3.5607 \\
\hline $\mathrm{H}$ & -1.4185 & -6.4372 & -1.6805 \\
\hline $\mathrm{H}$ & -3.7453 & -6.0852 & -5.2676 \\
\hline $\mathrm{H}$ & -2.8887 & -4.7993 & -0.5 \\
\hline $\mathrm{H}$ & -5.2148 & -4.4277 & -4.1410 \\
\hline $\mathrm{H}$ & -4.5910 & -3.1543 & -0.9272 \\
\hline C & 4.6591 & -4.3445 & -5.7638 \\
\hline C & 4.9737 & -4.9592 & -4.4241 \\
\hline 0 & 6.1267 & -5.0970 & -4.0152 \\
\hline C & 4.6075 & -2.8096 & -5.6492 \\
\hline $\mathrm{H}$ & 5.4486 & -4.6413 & -6.4589 \\
\hline $\mathrm{H}$ & 5.5621 & -2.4273 & -5.2757 \\
\hline $\mathrm{H}$ & 3.8209 & -2.4902 & -4.9580 \\
\hline $\mathrm{H}$ & 4.4101 & -2.3518 & -6.6230 \\
\hline $\mathrm{N}$ & 3.8883 & -5.2780 & -3.6812 \\
\hline C & 4.0314 & -5.7538 & -2.3246 \\
\hline C & 2.7458 & -5.6000 & -1.5197 \\
\hline C & 2.2146 & -4.1639 & -1.3698 \\
\hline C & 0.9792 & -4.1838 & -0.4661 \\
\hline C & 3.2779 & -3.2009 & -0.8252 \\
\hline $\mathrm{H}$ & 2.9638 & -5.1390 & -4.0586 \\
\hline $\mathrm{H}$ & 4.8450 & -5.1963 & -1.8568 \\
\hline $\mathrm{H}$ & 2.9341 & -6.0106 & -0.5199 \\
\hline $\mathrm{H}$ & 1.9585 & -6.2280 & -1.9631 \\
\hline $\mathrm{H}$ & 1.9060 & -3.7978 & -2.3597 \\
\hline $\mathrm{H}$ & 1.2532 & -4.4683 & 0.5557 \\
\hline $\mathrm{H}$ & 0.4990 & -3.2086 & -0.4138 \\
\hline $\mathrm{H}$ & 0.2266 & -4.8908 & -0.8327 \\
\hline $\mathrm{H}$ & 4.1388 & -3.1115 & -1.4958 \\
\hline $\mathrm{H}$ & 3.6373 & -3.5412 & 0.1514 \\
\hline $\mathrm{H}$ & 2.8619 & -2.1971 & -0.6982 \\
\hline C & 8.9924 & -5.7810 & -1.1681 \\
\hline C & 7.7678 & -4.8762 & -0.9628 \\
\hline C & 8.0825 & -3.4012 & -1.0948 \\
\hline C & 7.5794 & -2.6566 & -2.1700 \\
\hline C & 8.8870 & -2.7488 & -0.1502 \\
\hline C & 7.8663 & -1.2950 & -2.2889 \\
\hline C & 9.1778 & -1.3901 & -0.2687 \\
\hline C & 8.6656 & -0.6558 & -1.3398 \\
\hline $\mathrm{H}$ & 9.7845 & -5.5541 & -0.4468 \\
\hline $\mathrm{H}$ & 7.3418 & -5.0674 & 0.0311 \\
\hline $\mathrm{H}$ & 7.0110 & -5.1450 & -1.7036 \\
\hline $\mathrm{H}$ & 6.9757 & -3.1618 & -2.9180 \\
\hline $\mathrm{H}$ & 9.2735 & -3.3073 & 0.6988 \\
\hline $\mathrm{H}$ & 7.4647 & -0.7312 & -3.1271 \\
\hline $\mathrm{H}$ & 9.7829 & -0.8937 & 0.4839 \\
\hline$H$ & 8.8847 & 0.4042 & -1.4212 \\
\hline C & -2.6707 & 0.8417 & 0.6613 \\
\hline C & -1.4932 & 1.0319 & -0.0118 \\
\hline$c$ & -1.3379 & 2.2855 & -0.6913 \\
\hline 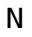 & -0.1958 & 2.6393 & -1.2682 \\
\hline & & & \\
\hline
\end{tabular}

\begin{tabular}{|c|c|c|c|}
\hline & -3.5052 & 866 & -0 \\
\hline & 627 & 711 & \\
\hline & 521 & 3900 & \\
\hline & 885 & 270 & \\
\hline & -4.6630 & 162 & -0 . \\
\hline & & 811 & \\
\hline & -0 . & -1 & -1 \\
\hline & -1 . & -1 & \\
\hline & -0 & 226 & \\
\hline & -0 . & 317 & -4 \\
\hline & -0. & 0.0374 & \\
\hline & 0.6088 & 140 & -1 \\
\hline & -0.0728 & 3.5669 & -1 \\
\hline & -0. & -0 . & \\
\hline & & & \\
\hline & -5.2 & 264 & \\
\hline & -4.3084 & 4.8141 & -0 \\
\hline & -5.3038 & 3.4565 & -1 . \\
\hline & -2.8697 & -0.0445 & \\
\hline & -1.8 & -2 & -0 \\
\hline & -2 & -2 . & -2 \\
\hline & -2 & -1 & -1 \\
\hline & 0. & 512 & -4 \\
\hline & -0.8 & -2 . & -5 \\
\hline & 1.8 & -3 & \\
\hline & 1. & -1 & \\
\hline & & -0 & \\
\hline & & -1 & \\
\hline & 2.7 & & \\
\hline & -8.1 & 55 & \\
\hline & -7.2 & & \\
\hline & -6 & & \\
\hline & -6 & & \\
\hline & -5 & & \\
\hline & -8 & & \\
\hline & -7.5737 & & \\
\hline & -7 & & \\
\hline & -6. & & \\
\hline & -0. & & \\
\hline & -0 . & & \\
\hline & & & \\
\hline & & & \\
\hline & 1.8 & & \\
\hline & 2.8 & & \\
\hline & 2. & & \\
\hline & & & \\
\hline & -0.8 & & \\
\hline & -1. & & \\
\hline & 0.3364 & & \\
\hline & & & \\
\hline & 3.3 & & \\
\hline & & & \\
\hline & & & \\
\hline & & & \\
\hline & & & \\
\hline & & & \\
\hline & & & \\
\hline & & & \\
\hline & & & \\
\hline & & & \\
\hline & & & \\
\hline & & & \\
\hline & & & \\
\hline & 4. & & \\
\hline & 5. & & \\
\hline & & & \\
\hline & -1.4 & -3 & \\
\hline & & & \\
\hline & & & \\
\hline & 11.8962 & & -1 \\
\hline & -10. & & -4 \\
\hline & -8.5 & & -4 \\
\hline & -0.6084 & & \\
\hline & -1.6 & 1 & \\
\hline & 6 & & 2 \\
\hline & & & \\
\hline & & -2 . & \\
\hline & & -1 & \\
\hline & & -3 & \\
\hline & & & \\
\hline & & & \\
\hline
\end{tabular}

\begin{tabular}{|c|c|c|c|}
\hline & 8.7215 & -6.8357 & \\
\hline & 9.4046 & 435 & \\
\hline & 442 & 070 & \\
\hline & 147 & 891 & \\
\hline & -1.2441 & -6.0566 & \\
\hline & & & \\
\hline & 2.0268 & & \\
\hline & -4.5892 & & \\
\hline & 2.2936 & 211 & \\
\hline & 475 & & \\
\hline & 0.6638 & & \\
\hline & 643 & & \\
\hline & -1. & & \\
\hline & -1 . & & \\
\hline & -1 . & & \\
\hline & -2 . & & \\
\hline & -3 & & \\
\hline & -2.7674 & & \\
\hline$c$ & -1.2767 & & \\
\hline C & -0. & & \\
\hline & -0 . & & \\
\hline & 45 & & \\
\hline & & & \\
\hline & 923 & & \\
\hline & 3. & & \\
\hline U & 3. & & -5 \\
\hline 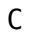 & & & \\
\hline & & & \\
\hline U & & & \\
\hline & & & \\
\hline & 3. & & \\
\hline & -1.3605 & & \\
\hline & -1.8838 & & \\
\hline & -2 . & & \\
\hline & -3 & & \\
\hline & -0. & & \\
\hline & & & \\
\hline & -1 & & \\
\hline & -1.0493 & & \\
\hline & -1.4242 & & -5 \\
\hline & -1 . & & \\
\hline & & & \\
\hline & -3 . & & \\
\hline & 2. & & \\
\hline & 2.2121 & & \\
\hline & 3.3058 & & -7 \\
\hline & 4. & & \\
\hline & 4. & & \\
\hline C & -5 . & -3 & \\
\hline C & -3 & -2 . & \\
\hline 0 & -3.4565 & $-1.5 e$ & \\
\hline$c$ & -5.9940 & -2 & \\
\hline 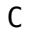 & -5 & -2 & \\
\hline & -6 . & -3 & \\
\hline & -4 & -2 . & \\
\hline & -2 . & -3 & \\
\hline$C$ & -1 & -3 & \\
\hline 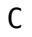 & -0.5927 & -2 & \\
\hline & -1.0069 & -2 & \\
\hline C & -0. & -4 & \\
\hline C & -1. & -5 & \\
\hline$C+2>$ & -2 . & -5 & \\
\hline & 0. & -1 . & \\
\hline & 272 & -1 . & \\
\hline C & 2.8622 & -2 & \\
\hline 0 & 2. & -3 & \\
\hline & 3. & -1 & \\
\hline$C$ & 5. & -1 . & \\
\hline & 5.8 & -1 & \\
\hline 0 & & -1. & \\
\hline C & 6.1857 & -1.1725 & \\
\hline 0 & 6.2005 & 0. & \\
\hline & 5. & -0 . & \\
\hline C & & & \\
\hline $\mathrm{H}$ & -7. & -2 & \\
\hline & כרחי & -1.3251 & \\
\hline $\mathrm{H}$ & -5.1057 & -4 & \\
\hline 1 & -0.7843 & -4 & \\
\hline & & -4 & \\
\hline & -1 . & -6 & \\
\hline & & -5 & \\
\hline
\end{tabular}




\begin{tabular}{|c|c|c|c|}
\hline H & -3.3127 & -5.5727 & 4.2607 \\
\hline ト & -3.1725 & -5.2643 & 2.5164 \\
\hline & -1.3144 & -2.4270 & 4.8549 \\
\hline & 1.6431 & -0.5021 & 2.0331 \\
\hline$\vdash$ & 1.1959 & -1.9810 & 1.1693 \\
\hline & 7.2045 & -1.5091 & 1.6226 \\
\hline & 5.8576 & -1.5133 & 0.4630 \\
\hline & 5.3865 & 0.6110 & 1.1026 \\
\hline & 5.3461 & -2.8661 & 2.4893 \\
\hline 卜 & 6.3526 & 0.2510 & 6.0032 \\
\hline$\Gamma$ & 2.3822 & 6.8142 & -3.7501 \\
\hline $\mathrm{F}$ & -4.3556 & 4.6388 & -4.0126 \\
\hline & -2.5769 & 4.5616 & -2.1253 \\
\hline 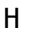 & 0.2447 & 8.0831 & -1.7496 \\
\hline $\mathrm{H}$ & 5.9636 & -1.3330 & 6.6878 \\
\hline & 4.7292 & -0.0365 & 6.7030 \\
\hline 1 & 4.0223 & -0.9758 & 4.7411 \\
\hline F & 0.8535 & -1.7657 & 4.0939 \\
\hline H & -7.5902 & -3.6454 & 0.9172 \\
\hline H & -6.5379 & -3.5357 & -0.4583 \\
\hline 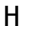 & 3.7250 & -0.4640 & 1.9962 \\
\hline $\mathrm{H}$ & -5.4786 & -3.2925 & 4.5376 \\
\hline C & -11.4651 & -4.8112 & 1.7 \\
\hline C & -10.3719 & -4.3617 & 0.7618 \\
\hline 0 & -9.1861 & -4.6008 & 1.0088 \\
\hline C & -11.2953 & -4.0412 & 3.0197 \\
\hline $\mathrm{N}$ & -10.7607 & -3.6327 & -0.3074 \\
\hline C & -9.7957 & -3.0287 & -1.2075 \\
\hline C & -8.6316 & 7.0341 & 3.6087 \\
\hline C & -8.5566 & 5.7639 & 2.7713 \\
\hline C & -8.5261 & 6.0050 & 1.2643 \\
\hline $\mathrm{N}$ & -8.0687 & 4.9301 & 0.5547 \\
\hline 0 & -8.9263 & 7.0391 & 0.7382 \\
\hline $\mathrm{H}$ & -10.2933 & -4.2100 & 3.4200 \\
\hline $\mathrm{H}$ & -12.0276 & -4.3632 & 3.7653 \\
\hline $\mathrm{H}$ & -11.4226 & -2.9654 & 2.8629 \\
\hline $\mathrm{H}$ & -12.4625 & -4.6529 & 1.2820 \\
\hline $\mathrm{H}$ & -9.2031 & -2.2563 & -0.7 \\
\hline $\mathrm{H}$ & -7.6918 & 5.1536 & 3.0400 \\
\hline $\mathrm{H}$ & -9.4372 & 5.1393 & 2.9694 \\
\hline $\mathrm{H}$ & -7.5346 & 4.2016 & 1.0276 \\
\hline $\mathrm{H}$ & -7.9332 & 5.0626 & -0.4366 \\
\hline $\mathrm{H}$ & -9.4720 & 7.6570 & 3.2915 \\
\hline $\mathrm{H}$ & -8.7500 & 6.7910 & 4. 6691 \\
\hline $\mathrm{H}$ & -7.7227 & 7.6335 & 3.4971 \\
\hline $\mathrm{H}$ & -11.3400 & -5.8844 & 1.8789 \\
\hline $\mathrm{H}$ & -10.3248 & -2.5790 & -2.0484 \\
\hline $\mathrm{H}$ & -9.1083 & -3.7888 & -1.5878 \\
\hline $\mathrm{H}$ & -11.7405 & -3.4325 & -0.4298 \\
\hline C & 4.2499 & 1.0607 & -1.1602 \\
\hline $\mathrm{H}$ & 4.0965 & 0.0509 & -1.5526 \\
\hline 0 & 3.9483 & 1.0259 & 0.2217 \\
\hline C & 5.7041 & 1.5089 & -1.3849 \\
\hline $\mathrm{H}$ & 6.3853 & 0.8382 & -0.8631 \\
\hline & 5.9681 & 1.5166 & -2.4446 \\
\hline & 5.8266 & 2.5198 & -0.9 \\
\hline
\end{tabular}

